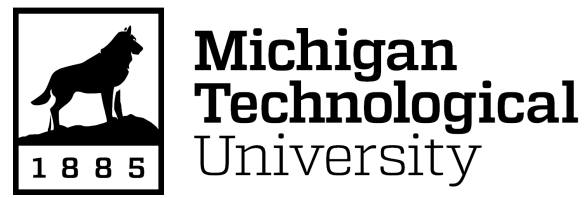

Michigan Technological University Digital Commons @ Michigan Tech

Dissertations, Master's Theses and Master's Reports

2021

\title{
A Resolution of Grain Boundary Strengthening Mechanisms by Nanoindentation Induced Local Mechanical Response
}

Prasad Pramod Soman

Michigan Technological University, ppsoman@mtu.edu

Copyright 2021 Prasad Pramod Soman

Recommended Citation

Soman, Prasad Pramod, "A Resolution of Grain Boundary Strengthening Mechanisms by Nanoindentation Induced Local Mechanical Response", Open Access Dissertation, Michigan Technological University, 2021.

https://doi.org/10.37099/mtu.dc.etdr/1216

Follow this and additional works at: https://digitalcommons.mtu.edu/etdr 


\title{
A RESOLUTION OF GRAIN BOUNDARY STRENGTHENING MECHANISMS BY NANOINDENTATION INDUCED LOCAL MECHANICAL RESPONSE
}

\author{
By \\ Prasad Pramod Soman

\begin{abstract}
A DISSERTATION
Submitted in partial fulfillment of the requirements for the degree of DOCTOR OF PHILOSOPHY

In Materials Science and Engineering
\end{abstract}

MICHIGAN TECHNOLOGICAL UNIVERSITY

2021

(C) 2021 Prasad Pramod Soman 
This dissertation has been approved in partial fulfillment of the requirements for the Degree of DOCTOR OF PHILOSOPHY in Materials Science and Engineering.

Department of Materials Science and Engineering

Dissertation Co-Advisor: $\quad$ Dr. Stephen A. Hackney.

Dissertation Co-Advisor: Dr. Erik G. Herbert.

Committee Member: Dr. Stephen L. Kampe.

Committee Member: Dr. John A. Jaszczak.

Department Chair: $\quad$ Dr. Stephen L. Kampe. 


\section{To Aai-Baba}




\section{Table of Contents}

List of figures.........................................................

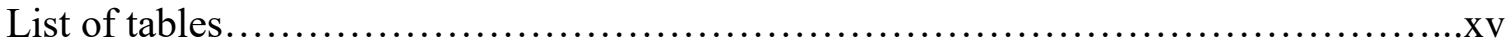

Preface.........................................................................

Acknowledgement............................................................

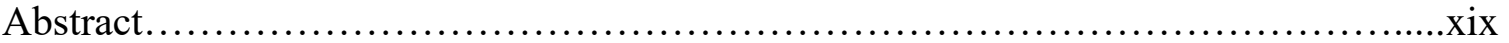

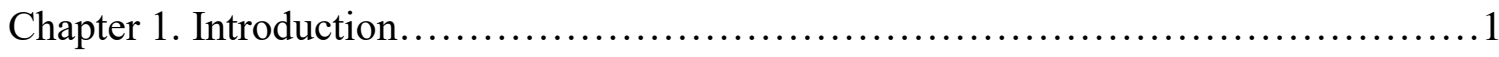

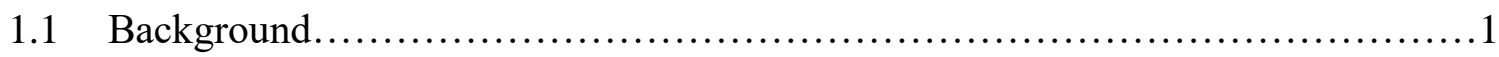

1.2 Literature Review....................................................

1.2.1 Review: Importance of sample preparation in nanoindentation.............3

1.2.2 Review: Grain boundary strengthening mechanisms....................4

1.2.3 Review: Grain boundary segregation studies.........................6

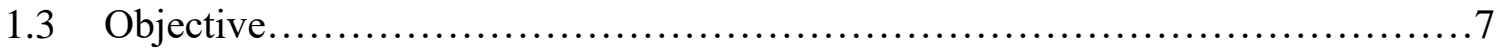

1.4 Hypotheses.........................................................

1.5 Design of experiments............................................. 10

1.6 Conclusion......................................................... 13

References............................................................ 15

Chapter 2. Effect of Processing on Nix-Gao Bilinear Indentation Results Obtained for

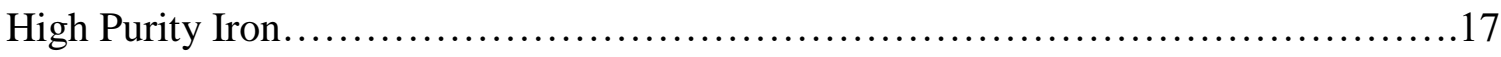

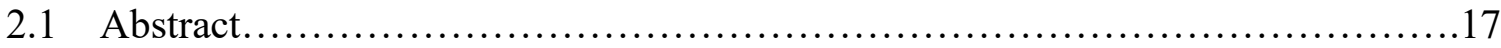

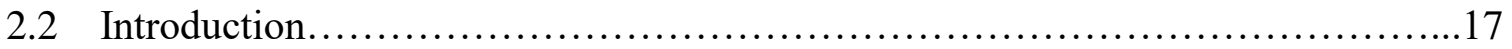

2.3 Experimental Work..................................................19

2.4 Result and Discussion................................................ 19

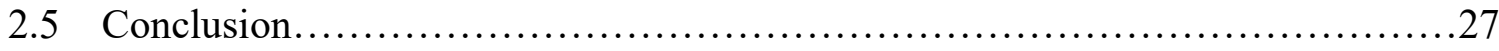




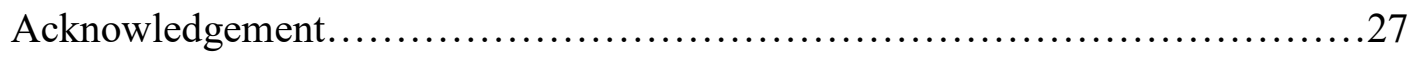

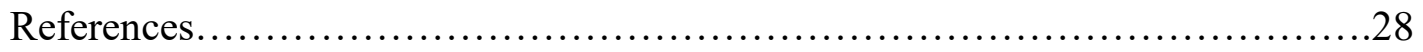

Appendix 2.A. Reprint Copywrite Permission.................................29

Chapter 3. Analysis of Local Grain Boundary Strengthening Utilizing the Extrinsic

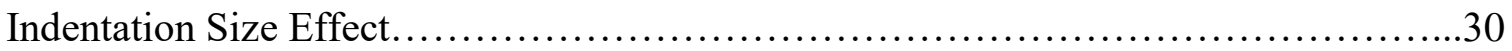

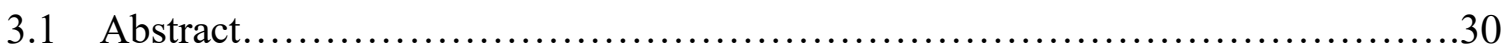

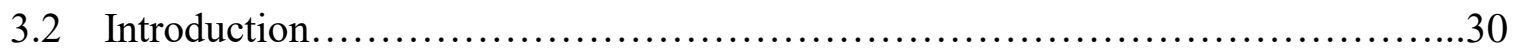

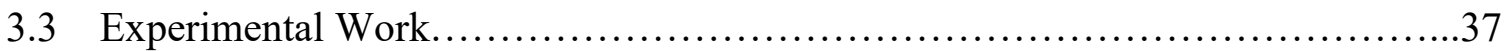

3.4 Result and Discussion....................................................... 45

3.4.1 Grain interior indentation results....................................45

3.4.2 Grain boundary indentation results................................50

3.4.3 Analysis: Continuum SGP formulation and the SGP boundary condition

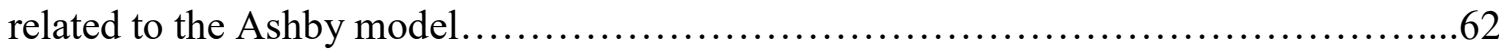

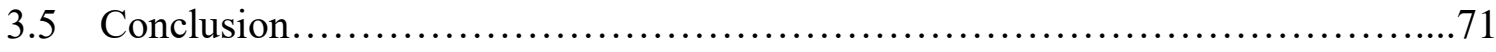

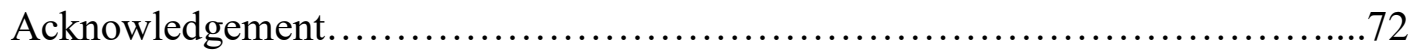

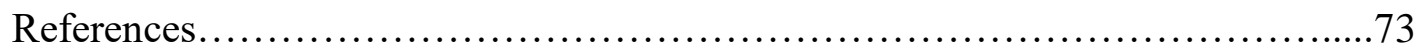

Appendix 3.A. Linearization of the Taylor Hardening flow stress relation..........81

Appendix 3.B. Consideration of the limiting case for the Schmid factor term,

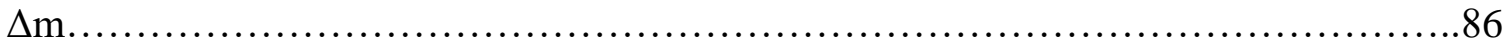

Appendix 3.C. Continuum SGP approximation...............................90

Appendix 3.D. Reprint Copywrite Permission..................................98

Chapter 4. Indentation Strain Rate Study of Grain Boundary Chemistry Effects.........104

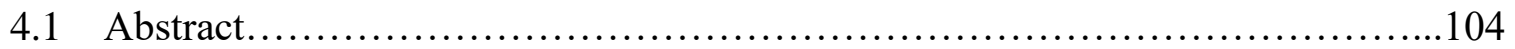




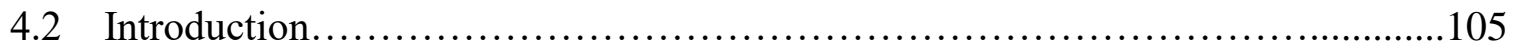

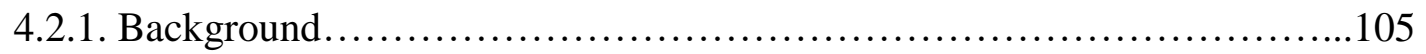

4.2.2. Indentation Strain-Rate Hardness Analysis................................111

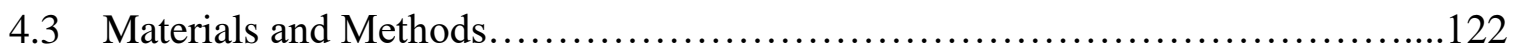

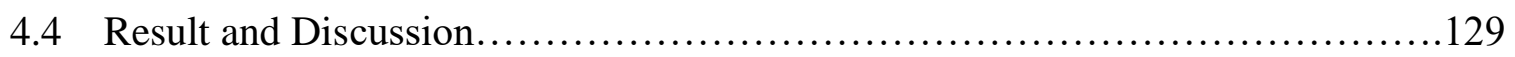

4.4.1 The Johnson-Cook analysis of the indentation hardness relaxation.........129

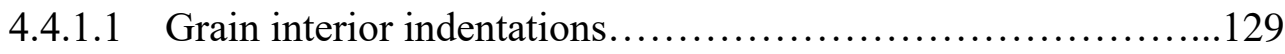

4.4.1.2 Grain boundary indentations.................................136

4.4.1.3 Local GB vs Remote GB indentations: JC and SGP model......152

4.4.1.4 Summary of grain boundary and grain interior results...........161

4.4.2 The analysis of grain boundary pop-in events using the Johnson-Cook

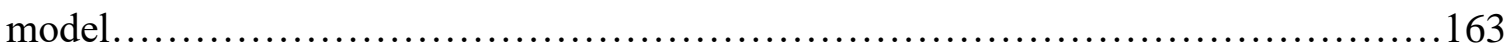

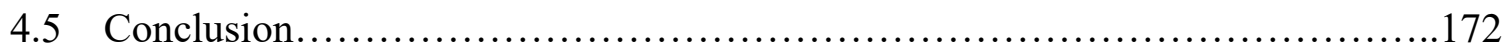

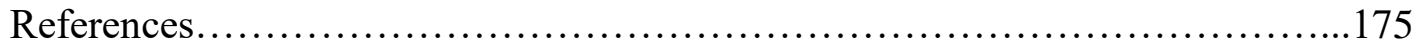

Appendix 4.A. Activation Volume Measurement................................182

Appendix 4.B. Nix Analysis for Mobile Dislocation Density Calculations.........186 


\section{List of figures}

Figure 1.1. Backscatter channeling contrast image of (a) Local grain boundary indentation: the indenter tip is positioned less than 0.5 micron from the grain boundary with the "wedge" of indenter being perpendicular to the grain boundary plane, (b) Remote grain boundary indentation: the indenter tip is positioned $\sim 2$ micron from the grain boundary and the flat face of the indenter parallel to the boundary .12

Figure 1.2. A representative hardness-depth curves for the local GB (blue), remote GB (pink) and adjacent grain interior (purple) indentation

Figure 2.1. (a) Representative P-h curves, (b) SEM image of an indentation, at $\sim 80^{\circ}$ tilt...20

Figure 2.2. Plot between hardness and depth (averaged from $20 \mathrm{H}$ vs h curves for each condition) for all the processing conditions, indicating presence of indentation size effect (ISE)

Figure 2.3. Nix-Gao plots for various processing conditions .22

Figure 2.4. (a) The hardness $-\log$ (diffusion length) plot of hardness measured at $150 \mathrm{~nm}$ and 500nm showing a typical logarithmic decay in flow stress with heat treat recovery time, (b) The reduction in bilinear behavior with annealing of surface polishing deformation is indicated as the $150 \mathrm{~nm}$ and $500 \mathrm{~nm}$ slopes approach one another .24

Figure 2.5. Plot of calculated forest dislocation density as a function of depth. The density is calculated assuming linear Nix-Gao ISE for the various processing conditions

Figure 2.6. (a) KAM, and (b) PQ metrics plotted against the logarithm of annealing duration .26

Figure 3.1. Figure 3.1. Backscatter channeling contrast image of (a) Remote grain boundary indentation: the indenter tip is positioned $\sim 2$ micron from the grain boundary and the flat face of the indenter parallel to the boundary, (b) Local grain boundary indentation: 
the indenter tip is positioned less than 0.5 micron from the grain boundary with the "wedge" of indenter being perpendicular to the grain boundary plane. .34

Figure 3.2. (a) Sample preparation methodology produces a large grain $(>1 \mathrm{~mm})$ sample with excellent quality map EBSD images, (b) KAM distribution plot for well-annealed surfaces, and (c) KAM images for mechanically polished surfaces that will include a contribution from the excess dislocations produced from the polish. (d) KAM plot for the mechanically polished surface and (e) well-annealed grain interior load:displacement curves showing the displacement discontinuity generally expected for dislocation nucleation process at surface with a very low preexisting dislocation density, (f) Secondary electron SEM image of a grain interior indentation The contact area is measured from the image and compared with the area function calculation from the reported indentation depth. The difference in the area measurement and area function result of $2.4 \%$ indicates the approximation of $h=h_{c}$ involves only a small error

Figure 3.3. (a) FIB cut on the grain boundary. (b) Fe electron diffraction shows no evidence of carbides or other second phase precipitates. (c) Local indentation grain boundary crosssection indicates grain boundary plane approximately perpendicular to the surface.

Figure 3.4. Backscatter SEM image for a linear array of closely spaced, shallow indentations with deeper indentations probing the properties using the local geometry similar to Figure 3.1(b).

Figure 3.5. (a) Representative P-h curves for mechanically polished (deformed) grain surface, well-annealed grain surface, well-annealed grain boundary surface intersection, and line source indentation. (b) Plot between hardness and depth (averaged for $20 \mathrm{H}$ versus $\mathrm{h}$ curves at each condition) for all the processing conditions, indicating presence of ISE as a function of processing condition. The hardness of the grain boundary is also shown on the plot for comparison. (c) Nix-Gao based $\mathrm{H}^{2}$ versus $1 / \mathrm{h}$ plots for all the processing 
conditions. (d) Calculated FD density [Eq. (3.2)] for as polished surface, 4 grain boundaries as well as line source indentations

Figure 3.6. (a) KAM map of surface deformation cross section. (b) Plan view KAM map of as-polished surface.

Figure 3.7. Representative hardness versus depth curves for 4 different grain boundaries in a well-annealed test specimen. (a) - (d) Show indentation results from the grain interior, the remote grain boundary indentation and the local grain boundary indentation for the carbon doped chemistry. (e) Comparison of local indentations for the same grain boundaries with and without carbon doping. (f) Comparison of local and remote grain boundary indentations with grain interior indentations with no carbon doping....

Figure 3.8. The comparison of the continuum SGP model Eq. (3.18) from the experimental results from local indentations on the 4 grain boundaries (a) - (d). Two experimental curves (dashed) from each boundary representing the variance of the behavior are presented. The

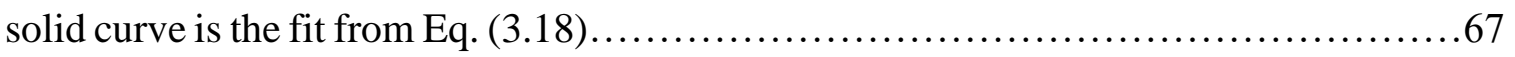

Figure 4.1. Backscatter channeling contrast image of (a) Local grain boundary indentation: the indenter tip is positioned less than 0.5 micron from the grain boundary with the "wedge" of indenter being perpendicular to the grain boundary plane, (b) Remote grain boundary indentation: the indenter tip is positioned $\sim 2$ micron from the grain boundary and the flat face of the indenter parallel to the boundary .................................. 110

Figure 4.2. Load-time history used for the nanoindentation experiments..............112

Figure 4.3. A representative curve for strain rate vs hardness with the experimental data and the Johnson-Cook model data............................................ 115

Figure 4.4. The EBSD based KAM and PQ data for annealed and deformed surface of iron. 
Figure 4.5. A representative load-depth plot with shallow depth pop-in, as a result of dislocation nucleation

Figure 4.6. Interference color of carbon film and corresponding film thickness when carbon deposition is performed on the standard brass substrate

Figure 4.7. A plot of volume diffusion coefficient vs temperature for $\mathrm{C}$ in $\alpha$-iron.

Figure 4.8. The Arrhenius diagram of grain boundary self and carbon impurity diffusion in $\alpha$-iron

Figure 4.9. Schematic of carbon concentration profile in $\mathrm{Fe}-\mathrm{C}$ system in equilibrium, indicating significant segregation at the grain boundary compared to adjacent grain interior, as predicted by the Gibbs Adsorption Isotherm. . .126

Figure 4.10. A schematic of carbon concentration gradient perpendicular to the grain boundary into the grain interior, predicted based on Whipple-Fisher contour angle measurement. 128

Figure 4.11. Activation volume variation as function of indentation strain rate. This result is very similar to bulk iron strain rate test analysis performed by F. A. Smidt.

Figure 4.12. Effective stress $\left(\sigma-\sigma_{0}\right)$ variation as a function of indentation strain rate.

Figure 4.13. (a) The characteristic strain rate variation as a function of indentation strain rate, for the grain interior in pure iron and carbon doped iron. (b) The predicted increase in mobile dislocation density by Neri et al. .

Figure 4.14. The athermal stress variation as a function of indentation strain rate, for the grain interior in pure iron and carbon doped iron 
Figure 4.15. A representative strain rate - hardness profile for the grain boundary and grain interior. 137

Figure 4.16. A representative hardness - depth profile for the grain boundary and grain interior indentation.

Figure 4.17. The Johnson-Cook analysis: activation volume vs characteristic strain rate for grain boundary and grain interior indentation with and without carbon, in (a) GB1, (b) GB2, (c) GB3, (d) GB4. The legend indicates grain boundary (GB) or grain interior (GI) in pure iron $(\mathrm{Fe})$ or carbon doped iron $(\mathrm{Fe}-\mathrm{C})$. The data is collected for indentation strain rate of $0.2 / \mathrm{s}$ in the loading segment. 138

Figure 4.18. The Johnson-Cook analysis: Athermal stress vs characteristic strain rate for grain boundary and grain interior indentation with and without carbon, in (a) GB1, (b) GB2, (c) GB3, (d) GB4. The legend indicates grain boundary (GB) or grain interior (GI) in pure iron $(\mathrm{Fe})$ or carbon doped iron $(\mathrm{Fe}-\mathrm{C})$

Figure 4.19. Mobile dislocation density vs athermal stress for grain boundary and grain interiors with and without carbon in (a) GB1, (b) GB2, (c) GB3, (d) GB4 ... 142

Figure 4.20. A log-log plot for activation volume vs hardness drop over constant load hold segment, where the activation energy can be determined using slope of the curve, (a) GB1, (b) GB2, (c) GB3, (d) GB4 145

Figure 4.21. Activation energy vs athermal stress based on statistical analysis GB data is different than GI data for (a) GB1, (b) GB2, (c) GB3, (d) GB4.

Figure 4.22. Evans-Kocks model-based critical stress as a function of indentation strain rate for the pure iron and carbon doped iron grain interior for GB1 .150 
Figure 4.23. The critical stress measured using Evans-Kocks model against the indentation strain rate, for grain boundaries and grain interior, with and without carbon, for GB1

Figure 4.24. A representative hardness-depth curves for the local GB (blue), remote GB (pink) and adjacent grain interior (purple) indentation 153

Figure 4.25. A representative hardness-time plot, Comparing similarly positioned local and remote GB indentation, where the remote indentation results are affected by the carbon doping whereas local indentation behavior appears independent of the grain boundary chemistry for (a) GB1 and (b) GB2... .154

Figure 4.26. Athermal stress vs characteristic strain rate for local and remote GB indentation, where local GB indentation data for with and without carbon is statistically indistinguishable unlike remote GB indentations with and without carbon, for (a) GB1 and (b) GB2.

Figure 4.27. Strain Gradient Plasticity model fitting for local GB indentations with and without carbon for (a) GB1, (b) GB2

Figure 4.28. A representative load-depth profile for grain boundary indentations with loading pop-ins

Figure 4.29. (a) Back-Scatter Electron (BSE) image of grain boundary indentation with remote indentation geometry relative to the grain boundary, (b) Schematic for cross section of Berkovich indenter [73], (c) Hardness vs depth profile for GB indent shown in (a), where pop-in occurs at a depth $\sim 685 \mathrm{~nm}$.

Figure 4.30. Experimental grain boundary pop-in data to show that the pop-in occurs as the indenter face crashes into the grain boundary in with and without carbon cases, (a) for GB1 and (b) GB2. 
Figure 4.31. Distance between GB - indenter tip (to determine theoretical depth of physical indenter-GB cross-over and the experimental depth of pop-in, for GB1 and GB2 .........166

Figure 4.32. A representative hardness-depth profile with hardness drop corresponding to the grain boundary pop-in................................................

Figure 4.33. Grain boundary relaxation event observed during indentation loading segment in case of (a) carbon doped iron, (b) pure iron. .168

Figure 4.34. Representative behavior of with and without carbon grain boundary pop-ins, based on the average data from "tanh" fitting, for (a) GB1 and (b) GB2 169

Figure 4.36. A representative hardness-depth and hardness-time profiles for grain boundary indentations with and without pop-in with reference to the grain interior..... 171

Figure 3.A.1. Percent error in Eq. (3.A(6)) when compared to Eq. (3.A(1)). The error is less than $1 \%$ when $\rho_{\mathrm{gb}}=0.1 \rho_{\mathrm{A}}$ and is $\sim 6 \%$ at $\rho_{\mathrm{gb}}=\rho_{\mathrm{A}}=10^{18} \mathrm{~m}^{-2}$.

Figure 3.B.1. Representative Nix-Gao plots for comparing grain boundary chopping indentation (green) with adjacent grain interior (black and orange) for (a) GB1, (b) GB2, (c) GB3, (d) GB4 .88

Figure 3.B.2. Correlating difference in hardness of adjacent grains (delta $H$ ) with the limiting case for $(\Delta \mathrm{m})$ Eq. 3. $\mathrm{B}(2)$. (b) Correlating difference in hardness between grain boundary and harder adjacent grain interior (delta $\mathrm{GH})$ with estimated $(\Delta \mathrm{m})$.

Figure 4.A.1. Activation volume as determined using Johnson-Cook model, Differential equation method for depth dependent activation volume and stress jump method for grain boundaries, grain interiors in pure, carbon doped and deformed conditions.... .184 
Figure 4.B.1. The normalized mobile dislocation density is plotted as a function of applied stress to show a predicted increase in mobile dislocation density with increasing

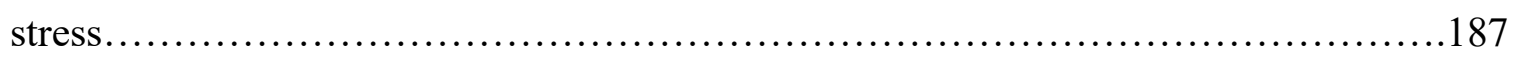

Figure 4.B.2. The calculated normalized mobile dislocation density at different values of

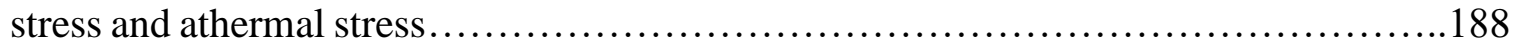




\section{List of tables}

Table 3.1. Analysis of intrinsic and extrinsic ISE related to specific grain boundaries......59

Table 3.2. Fit parameters for Eq. (3.18) in figure (3.8) ...........................66

Table 4.1. Compilation of Hall-Petch coefficients reported for pure metals and alloys, determined via nanoindentation technique

Table 4.2. Compilation of Hall-Petch coefficients reported for pure metals and alloys, determined via tensile testing.... 107

Table 4.3. The Johnson-Cook parameters and their physical interpretation using SchoeckGibbs Model and Evans-Kocks Model.

Table 4.4. Grain boundary and adjacent grain interior orientation details from the EBSD analysis .136

Table 4.5. Activation energy for the grain boundary and grain interior with and without carbon for GB1 145

Table 4.6. Activation energy for the grain boundary and grain interior with and without carbon for GB2.

Table 4.7. Activation energy for the grain boundary and grain interior with and without carbon for GB3 .146

Table 4.8. Activation energy for the grain boundary and grain interior with and without carbon for GB4

Table 4.9. SGP fitting parameters for local indentations on GB1 and GB2 for with and without carbon conditions. The fitting parameters are same for with and without carbon data, as shown in the figure 4.27. .158 
Table 4.10. Hyperbolic tangent function fitting parameters for the athermal stress vs time plots for GB1 with and without carbon..................................... 170

Table 4.11. Hyperbolic tangent function fitting parameters for the athermal stress vs time plots for GB2 with and without carbon................................... 170

Table 3.B.1. Limiting case analysis for the effective Schmid factor.....................87 


\section{Preface}

This dissertation includes 3 chapters that are part of my publications out of this project. Chapter 2 and 3 are already published in the MRS Advances Journal (DOI: 10.1557/adv.2018.135) and Journal of Materials Research (DOI: 10.1557/jmr.2019.102), respectively. Chapter 4 is manuscript draft that will be submitted for the publication soon.

In terms of contributions to the published work and in progress work, Professor Stephen Hackney, Professor Erik Herbert and Professor Katerina Aifantis has contributed immensely through insightful discussion leading to the analysis part of the work. All of them have contributed to the manuscript development process, as well. Praneeth Suddapalli needs to be credited for his contribution towards data analysis that goes in the chapter 4. 


\section{Acknowledgement}

First of all, I would like to thank Professor Hackney and Professor Herbert for their valuable mentorship, support and guidance throughout the project. They have always gone above and beyond to help me get through this journey.

I am also thankful to the contributions of Prof. Katerina E. Aifantis in this work.

My sincere thanks to Professor Kampe and Professor Jaszczak for agreeing to serve on my $\mathrm{PhD}$ committee. Their thoughtful insights during proposal preparations have been valuable.

The MSE department has played a key role in my success along this journey. I am honored to be a part of MSE family and would like to thank everyone in the MSE department. I would like to acknowledge contribution of Owen Mills, Dr. Dan Seguin, Dr. Joe Licavoli, Dr. Edward Laitila, Jerry Anzalone, Tom Wood, Paul Fraley, Elizabeth Miller, Ninad Mohale and Praneeth Suddapalli, to this project.

To all my friends and family away from home, Omkar, Ninad, Amruta, Chaitali, Adit, Akshada, Archana, Rohan, Nishal, Gayatri, Snigdha, Kartikeyan, Ramya, Prathamesh and many many others, thanks a lot for your support.

I would like to acknowledge financial support through the Department of Energy's office of Basic Sciences. I would also take this opportunity to thank Vaid, Tatkari, Vakure, Patankar, Phadke families for their support and Sakal Foundation, Chitpavan Sangh, and Shri Brihat Bharatiya Samaj for generous scholarships offered to begin my journey to US.

Last but not the least, I am totally indebted to all the sacrifices made by my family (Mom \& Dad, my beloved wife, Vaidehi, my elder brother, Anand and sister-in-law, Anushka and my lovely niece, Avani and my in-laws, Kale family), without which I can't even imagine this journey. I would also acknowledge valuable contributions of Soman family in this journey.

Thank you, God, and Gajanan Maharaj for everything! 


\section{ABSTRACT}

Grain boundary segregation is well known to cause significant embrittlement of alloys. But in certain cases, it has also been observed to increase mechanical strength. This project attempts to assess local mechanical behavior of specific grain boundaries with and without segregation in order to understand association between grain boundary chemistry and deformation mechanism utilizing instrumented nanoindentation technique. It is hypothesized that solute segregation strongly affects the grain boundary energy which in turn affects the deformation mechanism processes. This project also utilizes a unique ability provided by the instrumented indentation technique to interrogate local grain boundary strengthening mechanisms proposed by Hall-Petch and Taylor-Ashby using two different indentation geometries.

Grain boundary mechanical properties have typically been interpolated from macroscopic mechanical testing on polycrystalline materials, or alternatively, mechanical test procedures carried out on bulk bicrystals. The disadvantages to these types of studies relate to the difficulty in extracting the local response of a particular grain boundary (in the case of polycrystalline materials) or the grain boundary region (in the case of a bicrystal material) from the overall response of the complex interaction between the presence of the grain boundary and the deformation behavior far from the grain boundary. That is, the grain boundary causes a non-local response to the mechanical behavior. This non-local response is particularly evident in bicrystal deformation, where the macroscopic plastic displacement is inconsistent with that observed for single crystal deformation. Moreover, local hardness testing of grain boundary regions in macroscopically deformed materials show that the deformation in the grain boundary region is leads to greater local dislocation density than found in the grain center.

This project is designed to use nano-indentation to isolate the mechanical response of the grain boundary as the dependent variable, where indentation geometry, indentation 
rate, grain boundary misorientation and sample chemistry are the independent experimental variables. It is proposed that this approach can provide insight into long standing hypotheses regarding grain boundary strengthening mechanisms, including the Hall-Petch pile-up theory, grain boundary source theory, grain boundary layer theory and the Ashby-Taylor strain incompatibility theory. 


\section{CHAPTER 1: INTRODUCTION}

\subsection{BACKGROUND}

This research addressed a long-standing debate around grain boundary strengthening mechanisms through examination a local mechanical response of grain boundary. The instrumented indentation technique was utilized to interrogate the hypotheses regarding the grain boundary (GB) strengthening mechanisms, as interpreted via theories such as the Hall-Petch pile-up theory, the Ashby-Taylor strain incompatibility theory. Although grain boundary mechanical properties have been studied utilizing bulk mechanical testing or bicrystal testing, isolating the role of grain boundary has been an elusive goal. This project made a novel attempt by utilizing specific grain boundary indenter orientation and placement relative the boundary plane, namely Remote and Local GB indentations, to provide insight into the pile-up model vs local work hardening model for the grain boundary strengthening.

The indentation experiments were performed on 8 specific grain boundaries of varying twist-tilt characters, and corresponding adjacent grain interiors, on the high purity iron $(>99.999 \%)$ specimen. To understand the role of grain boundary, factors such as indentation geometry, indentation strain rate, grain boundary misorientation and sample chemistry were treated as the independent experimental variables.

While the instrumented indentation technique provides a unique ability to isolate individual microstructural component affecting material strength, such as grain boundary, grain interior, chemistry, it is very important to understand the necessity to ensure artifact free testing methodology. It can either be an instrumental artifact or the artifacts associated with the sample preparation process, that can lead to erroneous interpretation of indentation results. As this project relied extensively on the study of depth dependent hardness to characterize non-uniform deformation, the role of sample processing on nano-indentation 
results was examined for high purity $\alpha$-Fe. Indentation of surfaces with unresolved surface deformation due to mechanical polishing were compared to the same sample surfaces annealed at high temperature in a reducing atmosphere. Behaviors of interest included any variance in the indentation size effect. The differences in indentation behavior with annealing time and temperature are correlated with EBSD as an independent measure of surface layer crystallinity. The results were interpreted with the Nix-Gao model, using a depth dependent statistically stored dislocation density to rationalize the nonlinear experimental results.

The concept of extrinsic indentation size effect (ISE) observed in case of nonuniformly deformed grain interior of pure iron is utilized to analyze the depth dependent hardness for Berkovich indentation of non-uniform dislocation distributions with one and two dimensional deformation gradients and is then extended to indentation results at grain boundaries. The role of the Berkovich pyramid orientation and placement relative to the grain boundary on extrinsic ISE is considered in terms of slip transmission at yield and plastic incompatibility during post yield deformation. The results are interpreted using a local dislocation hardening mechanism originally proposed by Ashby, combined with the Hall-Petch equation. The Hall-Petch coefficient determined from the extrinsic ISE of the grain boundary is found to be consistent with published values for pure Fe and mild steel. A simple, linear continuum strain gradient plasticity model is used to further analyze the results to include contributions from a non-uniform distribution in plastic strain and dislocation density.

This project further investigated the local mechanical behavior of pure iron with and without the influence of a grain boundary segregation through carbon doping on 4 general grain boundaries. In this study, local deformation was carried out using nano-indentation with varying indentation-grain boundary geometry and strain rate. Stress relaxation under constant load and strain rate response to stress jumps were used to determine activation energy, activation volume and athermal stress as a function of grain boundary chemistry. 
This study facilitated examination of chemistry effects in grain boundary deformation, in the context of Hall-Petch dislocation pile up model vs Taylor-Ashby strain incompatibility model.

\subsection{LITERATURE REVIEW}

\subsubsection{Review: Importance of Sample Preparation in Nanoindentation}

The instrumented indentation technique provides a unique ability to isolate and assess local mechanical properties of various microstructural features (such as grain boundary, grain interior, chemistry) at a very small length scale. But as with any other characterization technique, accuracy and reproducibility of results obtained via nanoindentation tests depends largely on the quality of sample being tested. The effect of factors, such as sample roughness, tilt, thermal equilibrium, specimen mounting and positioning, pile-up/sink-in effects, on nanoindentation test results are well documented in a literature. In this work, all these parameters that could result in experimental artifacts are carefully considered.

This work utilizes well established Nix-Gao model to characterize dislocation free sample surface preparation. This model was developed to provide physical insight into the mechanism responsible for commonly observed phenomenon, Indentation Size Effect, which is manifested through nanoindentation based hardness-depth profile where hardness drops as a function of depth. Amongst various theories to explain Indentation Size Effect, Nix-Gao model expands upon the concept of geometrically necessary dislocations (GNDs) in addition to the statistically stored dislocations (SSDs) that are necessary to explain drop in hardness as a function of depth. Based on this model, if the material being tested in nanoindentation is free of any surface deformation, then $\mathrm{H}^{2}$ vs $1 / \mathrm{h}$ plot (Nix-Gao plot) would appear linear in nature. It is shown that the sample preparation of indentation experiments shouldn't only be limited to mechanical polishing but should include stress relieving annealing heat treatment at $850^{\circ} \mathrm{C}$ for $4-5$ hours. The presence of sub-surface 
work hardening layer upon metallographic polishing can be detected by the bilinear nature of Nix-Gao plot as a linearity predicted by the original model relies solely on GNDs and SSDs to explain ISE observed in crystalline materials.

In the chapter 2, the effect of surface deformation induced by polishing on Nix-Gao non-linearity (bilinear behavior) is examined which is a result of mechanical polishing introduced 'forest dislocations' (FD) required for accommodation of indentation plasticity, in addition to the GNDs and SSDs. This approach leads to the rather simple, but previously unexplored, concept that the spatial variation of FD (due to mechanical polishing) with depth can contribute to bilinear Nix-Gao behavior [8].

\subsubsection{Review: Grain Boundary Strengthening Mechanisms:}

It was Hall and Petch who provided seemingly simple mathematic formulation to relate grain size with the strength of materials. Although the Hall-Petch equation (given below) appears to consider only grain boundary effects and not other defect structures or microstructural features towards the strength of metals and alloys, it has been of enormous importance in the field of metallurgy due to ability to relate experimental observations in wide ranging pure metals and alloys over past several decades. The Hall-Petch equation led to development of variety of models to explain underlying mechanisms responsible for grain size-strength association.

$$
\sigma_{y}=\sigma_{o}+k d^{-1 / 2}
$$

Where, $\sigma_{y}$ - yield strength, $\sigma_{o}$ - friction strength, $k-$ Hall-Petch coefficient, $d-$ grain diameter.

Although it may appear that Hall-Petch equation only highlights the role of grain boundary in the strengthening mechanism, it is believed that first part on a right of the 
equation captures physics associated with a contribution of grain boundary whereas second term summarizes effects of other parameters such friction forces.

Amongst variety of models presented here, the first one was envisioned by Hall where he proposes pile-up-based strengthening. According to this model, dislocations generated within a grain as a result of deformation process tend to push against the grain boundary. As a result of this pile up, if the critical stress limit is reached, it would result in either dislocation emissions across grain boundary or nucleation of the dislocations in the adjacent grain. As the amount of dislocations accommodated within grain is limited by grain size, experimental observations of relationship between grain size and strength appears to consistent with this model. Around 1967, Armstrong [9] was first to hypothesis that Hall-Petch coefficient would be function of chemistry in addition to misorientation. Later it was experimentally shown that Hall-Petch coefficient appeared to vary with alloy chemistry. Hall's pile-up model failed to explain these observations paving pathways for alternative explanations.

Later several other models came along which relied on strain hardening effects. First of such model was presented by Li proposing the grain boundary ledges as a dislocation source at yielding. This model also possessed similar mathematical form but failed to provide any direct evidence, similar to the pile-up model.

The most acceptable work hardening model was proposed by Ashby. The concept is based on the plastic strain incompatibility between adjoining grains. Ashby proposes that as a polycrystalline material undergoes deformation, unlike single crystal behavior, depending on grain orientation, slip system availability, each grain undergoes nonhomogeneous plastic flow, due to the constraint factors. While maintaining a structural homogeneity of the material, due to strain incompatibility between grains, excessive dislocation generation is warranted. In addition to the dislocations associated with the overall uniform plastic deformation of the material i.e. statistically stored dislocations (SSD), Ashby introduced a concept of geometrically necessary dislocations (GND). These 
dislocations are formed at the interfaces between grains to accommodate for the varying amount of plastic displacement. Ashby's equivalence of Hall-Petch equation can be written as:

By comparing both equations, it can be seen that Hall-Petch coefficient K, doesn't solely depend of the grain size but also considers strain effects.

Despite widely accepted Taylor-Ashby approach as well as other models, variation in Hall-Petch coefficient in pure metals and alloys couldn't be explained by the single theory or models. Chapter 3 and 4 discuss a novel method to utilize remote and local grain boundary indentation geometries to study Hall-Petch pile-up model and Taylor-Ashby based strain incompatibility model using nanoindentation technique to gain insight into underlying physics.

\subsubsection{Review: Grain Boundary Segregation Studies:}

Traditional approach to investigate grain boundary contribution to material strength has largely relied on macroscopic testing such as tensile tests. It was initially believed that Hall-Petch coefficient, $\mathrm{K}$ is primarily a function of misorientation across grain boundary. But later several research studies on different pure metals and alloys such as $\mathrm{Fe}-\mathrm{C}, \mathrm{Fe}-\mathrm{N}$, Fe-P, Fe-C-P, Cu-Zn, Cu-Ni-Zn, Cu-Al, indicated strong correlation between Hall-Petch coefficient and solute concentration [2, 9, 10, 11, 12, 13, 14]. All these studies have primarily utilized tensile testing. Even recent advances in grain boundary studies utilizing nanoindentation technique to study local mechanical responses have established similar observations. Some of these examples presented in $[10,11]$ where slightest of carbon, nitrogen solute concentration [30ppm to $60 \mathrm{ppm}$ ] has a significant variation in $\mathrm{K}$ [358 $\left(\mathrm{Mpa}_{\mathrm{um}}^{\wedge}-1 / 2\right)$ to $\left.516\left(\mathrm{Mpa}^{\mathrm{u}}{ }^{\wedge}-1 / 2\right)\right]$ values, when alloyed with Fe. D. Akama [15] conducted a study on IF steel with $\mathrm{Ni}$ and carbon as alloying elements to estimate the effect solute has on the Hall-Petch coefficient. This study discusses yield point phenomenon observed in case of steel alloyed with carbon or Ni as alloying constituents. According to 
the Cottrell atmosphere theory, the solute particles are locked by the 'Cottrell atmosphere' formed by the solute and at critical load, the dislocation is removed from the atmosphere to cause yielding. This research group proposes that this could possibly be due to stabilizing effect alloying elements have on the grain boundary. Although the researchers recognize the contribution of the grain boundary deformation, examination of specific boundaries with and without alloying content has not yet been attempted. Another research group led by Takeda [10], has investigated IF steel with carbon \& nitrogen alloying, to study yield point phenomenon. Their work concludes that it depends on solute tendency to segregate at the grain boundary whether yield point phenomenon will be observed in the alloy. Despite establishing relationship between $\mathrm{K}$ and chemical composition, the physics behind the mechanism responsible for contribution of chemical composition.

\subsection{OBJECTIVE}

It is well known that indentation at grain boundaries produces a strengthening effect, as expected from macro-deformation studies of polycrystalline materials. However, the localized nature of indentation deformation has not been utilized to assess the various mechanisms of grain boundary strengthening (Hall-Petch, Ashby, Li boundary source). It is the purpose of the proposed work to take advantage of the nano-indentation stress field geometry to interpret the grain boundary hardness in terms of the microstructural aspects of grain boundary strengthening models. 


\subsection{HYPOTHESES}

1.4.1 The Nix-Gao model can be used to ensure that a specimen used for nanoindentation experiment is free of any sub-surface defects introduced by mechanical polishing process. If the Nix-Gao plot i.e. $\mathrm{H}^{2}$ vs $1 / \mathrm{h}$ exhibits deviation from predicted linear profile, then it can be associated with the presence of forest dislocations underneath the specimen surface as a result of work hardening due to mechanical polishing process because the Nix-Gao assumes defect free surface to predict linear $\mathrm{H}^{2} \mathrm{vs} 1 / \mathrm{h}$ profile, where solely Statistically Stored Dislocations (SSD) and Geometrically Necessary Dislocations (GND) contribute to the hardness measurement as a function of depth.

1.4.2 If the indentation is 'local' to the grain boundary, then the dislocation formation occurs almost simultaneously in both grains. If the local indentation results in simultaneous plastic deformation of the grains on either side of the grain boundary, having a rigid sample-indenter interface to constrain surface relaxation, then there is a plastic incompatibility across the grain boundary (Ashby) that results in a hardening response that is inverse to indentation depth. The inverse depth dependence on the hardness is expected because the Ashby strain gradient theory predicts that the geometrically necessary dislocation density decreases as the stressed volume containing the grain boundary increases in size.

Test of hypothesis.

- Constant load Johnson-Cook interpretation of hardness-strain rate curves.

- Effects of change of boundary chemistry. 
1.4.3 The 'remote' indentation geometry results in dislocation multiplication $>=1 \mathrm{um}$ away from the grain boundary and dislocation formation at small indentation depths in the adjacent grain has a relatively low driving force. If the indentation geometry is 'remote' to the grain boundary, then the increase in hardness with increasing indentation depth occurs (relative to the grain interior) because of the grain boundary barrier to slip transmission (i.e. Hall-Petch).

Test of Hypothesis.

- Constant load Johnson-Cook interpretation of hardness-strain rate curves. Should be different than in remote indentation.

- Effects of change of boundary chemistry. Should be different than in remote indentation.

In order to successfully test the hypotheses, following sub-objectives need to be accomplished:

- With the main goal to be able to isolate the local grain boundary responses as a result of a complex state of stress induced by nanoindentation experiment, it is imperative that specimen is preparation is meticulously done, to avoid possibilities of experimental or non-material artifacts influencing the data interpretation.

- Indentation experiments performed on the same specimen but with different processing conditions, i.e. only mechanically polished surface, mechanically polished plus partially annealed surface, and well-polished well-annealed surface, re-establish the need for well-designed sample preparation process, as indentation size effect gradually vanishes for well-prepared well-annealed specimen.

- This requires thoughtful development of the sample preparation procedure and a careful characterization using SEM, EBSD, and nanoindentation, as explained in the experimental procedure section. 
- Further, although it is well established knowledge that the grain boundary is stronger relative to its adjacent grain interior, it is necessary to validate this observation by performing an indentation experiments in the grain interior and at the grain boundary.

- Addressing the primary goal to investigate the grain boundary strengthening mechanisms responsible for the observed grain boundary hardening relative to the interior, indentations are performed at the boundary with a specific grain boundary indenter orientation and positioning relative to the boundary, namely remote and local.

- Johnson-Cook model in conjunction with Schnoeck- Gibbs model is utilized analysis constant load hold segment data for all the indentations performed, to estimate parameters such as activation volume, activation energy, mobile dislocation density, and athermal stress.

- Several indentation-based control experiments are carried out, to validate the application of Johnson-Cook model, to test the hypothesis regarding local and remote indentations.

\subsection{DESIGN OF EXPERIMENTS}

1. Experiments to test the grain boundary hardening relative to the adjacent grain interior:

For well-prepared indentation ready specimen of pure iron, indentation experiments were performed on a specific grain boundaries and corresponding grain interiors. Matrix of 20 indents with $30 \times 30$ micron spacing was performed in the grain interior and 20 indentations were performed on the grain boundary. These grain boundary indentations include remote as well as local, as the purpose here is to examine grain boundary behavior against grain interior. This is well established that grain boundary, due to misorientation between adjacent grain interiors, is a high energy structure and acts as an obstacle for dislocation transmission from one grain to another. The analysis section will include all the test results for the grain boundary- grain interior comparison, 
where it is observed that both types of grain boundary indentations i.e. remote and local, exhibit higher hardness compared to its neighboring grain interior.

Following experiments are performed in total on a given grain boundary, GB1:

- Number of Grain boundary indentation - Pure Fe at $0.2 / \mathrm{s}-20$

- Number of Grain boundary indentation - Fe-C at 0.2.s - 20

- Number of Grain boundary indentation - Pure Fe at 4/s - 20

- Number of Grain boundary indentation $-\mathrm{Fe}-\mathrm{C}$ at $4 / \mathrm{s}-20$

- Number of Grain interior indentation - Pure Fe at $0.2 / \mathrm{s}-20$

- Number of Grain interior indentation - Fe-C at 0.2.s - 20

- Number of Grain interior indentation - Pure Fe at 4/s - 20

- Number of Grain interior indentation $-\mathrm{Fe}-\mathrm{C}$ at $4 / \mathrm{s}-20$

Grain boundary indentations include remote and local indentations and grain interior indentations include both grain interior across GB. All these experiments will be performed on the total of 8 grain boundaries with varying tilt-twist nature.

2. Experiments to test the indention geometry effects:

As mentioned previously, the grain boundary indentations are performed with 2 specific grain boundary - indenter positioning, namely remote and local [Fig 1.1 (a) and (b)]. The remote and local indentation have specific Hardness vs depth profile associated with each other where size effects is prominent in case of local indentation, and gradual hardening relative to the interior is observed for the remote indentation. It is proposed that the hardening behavior observed for these 2 types of the grain boundary [Fig 1.2], are due to activation of unique mechanisms in each case, i.e. pile-up based and strain incompatibility. To ensure that observed difference in the hardness vs depth profiles for the local and remote indentations has statistically significant, numerous experiments were performed on the same grain boundary under different test conditions such as strain rates 
(0.2/s and 4/s), sample chemistry (with and without carbon), as discussed in the analysis section.

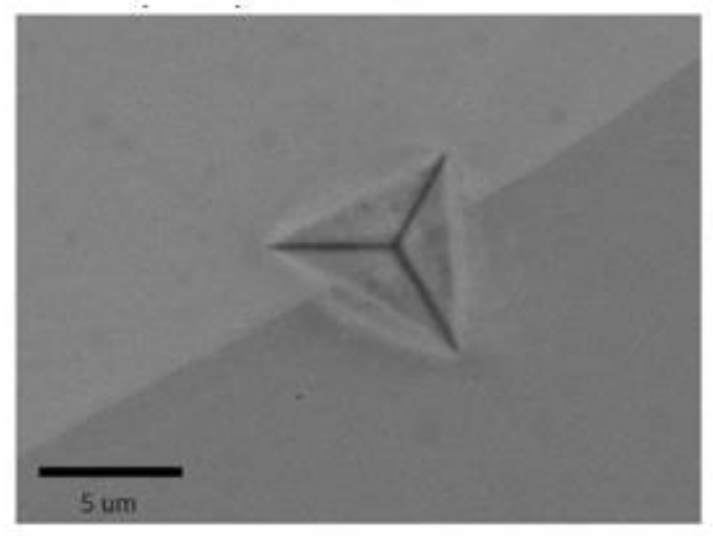

(a)

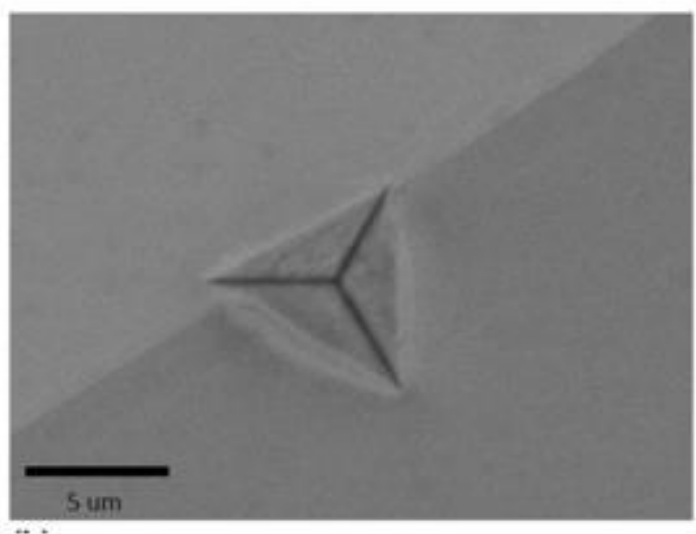

(b)

Figure 1.1: Backscatter channeling contrast image of (a) Local grain boundary indentation: the indenter tip is positioned less than 0.5 micron from the grain boundary with the "wedge" of indenter being perpendicular to the grain boundary plane, (b) Remote grain boundary indentation: the indenter tip is positioned $\sim 2$ micron from the grain boundary and the flat face of the indenter parallel to the boundary. [1] 


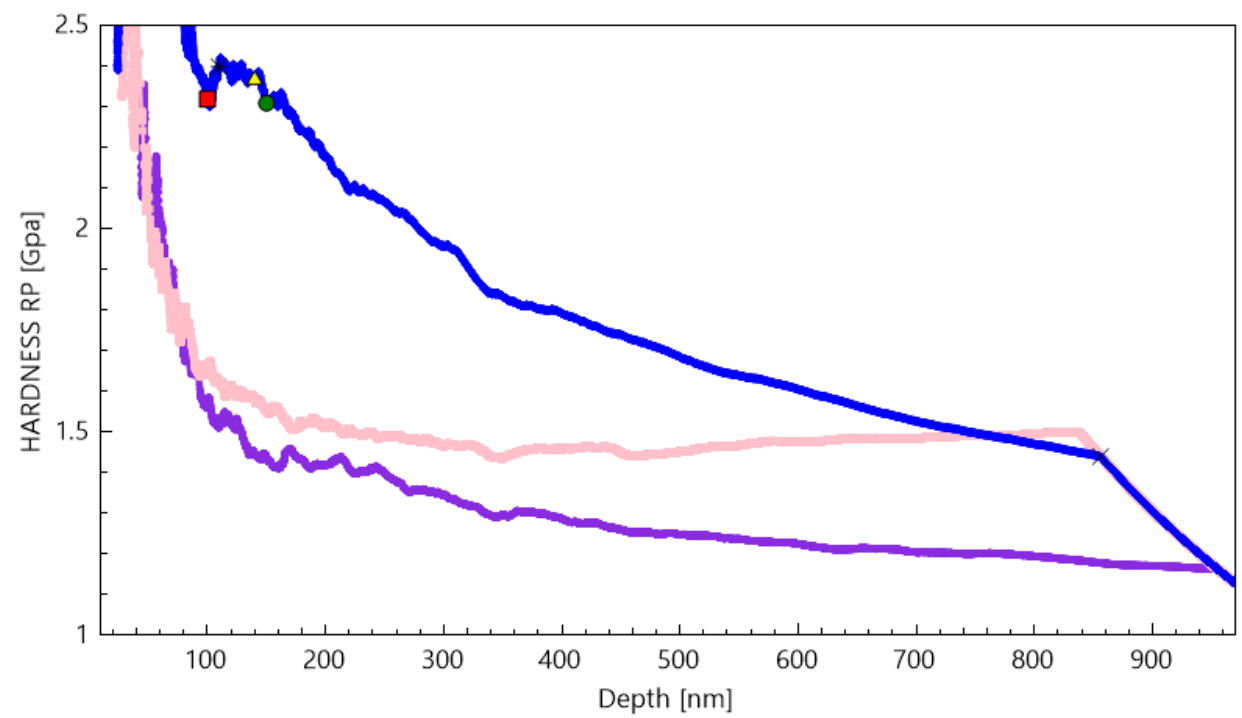

Figure 1.2: A representative hardness-depth curves for the local GB (blue), remote GB (pink) and adjacent grain interior (purple) indentation.

\subsection{CONCLUSION:}

To summarize, this chapter provides a foundation for the grain boundary geometry and chemistry effects on the strengthening mechanisms observed in $\mathrm{Fe}-\mathrm{C}$ and BCC metals in general.

In chapter 2, an importance of the sample preparation is emphasized. The chapter discusses various parameters that can contribute to experimental artifacts and eventually erroneous observations. The Nix-Gao model is primarily utilized to ensure that the specimen used during indentation experiments is free from any surface defects that can influence material property measurements.

Further chapter 3 discusses grain boundary indentation experiments where remote and local indentations are discussed in a great detail in comparison to the adjacent grain interior indentation results to discuss associated grain boundary hardening mechanisms. Although this chapter is mainly dedicated to the concepts introduced by Taylor and Ashby 
regarding localized dislocation density enhancement due to strain incompatibility, it highlights long standing hypothesis regarding grain boundary strengthening by TaylorAshby model vs Hall-Petch pile up model. In this chapter hypothesis associated with the local grain boundary indentations is discussed and associated chemistry effects are studied.

Finally, chapter 4 focusses on the grain boundary hardening observed in remote grain boundary indentation from the perspective of Hall-Petch pile up model. This chapters provides a novel approach to investigate remote grain boundary indentations where Johnson-Cook model is utilized. Previous studies have relied on the grain boundary popin behavior during indentation loading segments to investigate grain boundary yielding phenomenon and have attempted to associate grain boundary segregation. But use of Johnson-Cook model, to study relaxation behavior and chemistry effects on the grain boundary indentations is first of its kind attempt, as per authors knowledge. 


\section{REFERENCES:}

1. Analysis of local grain boundary strengthening utilizing the extrinsic indentation size effect, Soman, P., Herbert, E., Aifantis, K., \& Hackney, S., Journal of Materials Research, 34 (13), 2347-2369, 2019.

2. The deformation and ageing of mild steel: III discussion of results, Hall, E.O., Proc. Phys. Soc. B 64, 747, 1951.

3. The cleavage strength of polycrystals, Petch, N. J., Iron Steel Inst. 174, 25, 1953.

4. Petch relation and grain boundary sources, Li, J.C.M., Trans. Metall. Soc. AIME 227, 239, 1963.

5. Hall-Petch Relationship and Dislocation Model for Deformation of Ultrafine-Grained and Nanocrystalline Metals, M. Kato, Materials Transactions 55 (1), 19-24, 2014.

6. The deformation of plastically non-homogeneous materials, Ashby, M.F., Philos. Mag. 21, 399, 1970.

7. Plastic strain in metals, Taylor, G., J. Inst. Met. 62, 307, 1938.

8. Effect of Processing on Nix-Gao Bilinear Indentation Results Obtained for High Purity Iron, Soman, P., Herbert, E., Aifantis, K., Hackney, S., MRS Advances, 3(8-9), 477486, 2018.

9. The plastic deformation of polycrystalline aggregates, Armstrong, R.W., Codd, I., Douthwaite, R.M., and Petch, N.J., Philos. Mag. 7, 4558, 1962.

10. Effect of interstitial elements on Hall-Petch coefficient of ferritic iron, K. Takeda, N. Nakada, T. Tsuchiyama, S. Takaki, ISIJ International 48 (8), 1122-1125, 2008.

11. Effect of phosphorous on Hall-Petch coefficient in ferritic steel, N. Nakada, K. Takeda, T. Tsuchiyama, S. Takaki, ISIJ International 51 (7) 1169-1173, 2011.

12. Ductile-brittle transition in steels irradiated with neutrons, Hull, I. L. Mogford, The Philosophical Magazine: A Journal of Theoretical Experimental and Applied Physics, 3 (35), 1213-1222, 1958.

13. Creep in face-centered cubic metals with special reference to copper, P. Feltham, J. D. Meakin, Acta Metallurgica 7(9), 614-627, 1959. 
14. The effect of temperature and grain size on the yield point of copper solid solutions, W. Koster and M. O. Speidel, Z. Metallkunde 56, 585, 1965.

15. Discontinuous yielding induced by the addition of nickel to interstitial-free steel, D. Akama, N. Akada, Scripta Mat., 82, 13-16, 2014. 


\section{CHAPTER 2: EFFECT OF PROCESSING ON NIX-GAO BILINEAR INDENTATION RESULTS OBTAINED FOR HIGH PURITY IRON}

Published in MRS Advances - DOI: 10.1557/adv.2018.135

\subsection{ABSTRACT}

Instrumented indentation of a high purity $\mathrm{Fe}$ surface with unresolved surface deformation due to mechanical polishing is compared to the same grain surface annealed at increasing time and temperature. The differences in indentation size effect behavior with annealing are correlated with hardness and electron backscatter diffraction measurements as independent measures of surface layer deformation. It is found that the Nix Gao plot evolves from nonlinear (bilinear) towards the predicted linear relationship as the surface deformation is removed. The experimental observations are rationalized by inclusion of a depth dependent, polishing induced forest dislocation density within the Nix-Gao model.

\subsection{INTRODUCTION}

The indentation size effect has been studied for variety of materials reporting significant variation in hardness as a function of depth [1-3]. To explain mechanism responsible for the indentation size effect, various strain gradient plasticity [4-5] and mechanistic models [6-8] have been proposed. Nix and Gao [6] proposed, arguably the most widely accepted mechanistic model, based on geometrically necessary dislocations (GND) required for the indentation plastic shape change and statistically stored dislocations (SSD) due to the indentation characteristic strain [6]. To explain decreasing hardness with increasing depth, this model proposes that GND have an increase in spacing as the indentation depth increases. The Nix Gao model for indentation of initially defect free material relates the hardness to the indentation induced dislocation density as: 


$$
\begin{gathered}
H=H_{0} * \sqrt{1+\frac{h^{*}}{h}} \\
H_{o}=3 \sqrt{3} \alpha G b \sqrt{\rho_{S S D}}, h^{*}=\frac{3 \tan ^{2} \theta}{2 b \rho_{S S D}}, \rho_{G N D}=\frac{3 \tan ^{2} \theta}{2 f b h}
\end{gathered}
$$

Where, $\alpha$-constant $=0.5$, G- Shear modulus, b- Burger's vector, $\rho_{G N D}$ - GND density, $\rho_{S S D}$ - SSD density, $\theta$ - angle between indenter and un-deformed surface , $h^{*}$ Characteristic depth, $f$ - material dependent factor for volume correction [9].

According to the simplest interpretation of the Nix-Gao indentation size effect theory, the slope of hardness squared $\left(\mathrm{H}^{2}\right)$ plotted against inverse depth $(1 / \mathrm{h})$ should be determined by the geometry of the indenter and the volume of the plastic zone under the indenter. For a Berkovich indenter, this slope is predicted approximately constant for hardness at indentation depths that exceed depths effected by tip defect contributions and contributions due to energy barriers for dislocation nucleation. This type of linear behavior has been observed in a number of carefully prepared materials such as $\mathrm{Cu}$ [2] and $\mathrm{Ag}$ [3]. However, there have been multiple reports of so called 'bilinear' behavior, reviewed in [8], in which the Nix-Gao plots show measurable deviation from linearity. That is, the slopes of $\mathrm{H}^{2}$ vs $1 / \mathrm{h}$ at small indentation depth are observed to be significantly different (less) than the slopes at large indentation depths. This paper examines the effect of surface deformation induced by polishing can have on Nix-Gao non-linearity (bilinear behavior), noting that mechanical polishing introduces 'forest dislocations' (FD), which will be in addition to the SSD and GND's required for accommodation of indentation plasticity. This approach leads to the rather simple, but previously unexplored, concept that the spatial variation of FD (due to mechanical polishing) with depth can contribute to bilinear NixGao behavior. 


\subsection{EXPERIMENTAL PROCEDURE}

A high purity (99.999+) D- Iron specimen $10 \mathrm{~mm}$ diameter by $4 \mathrm{~mm}$ thickness was cut from as-drawn rod purchased from Goodfellow USA. The specimen was annealed and then mechanically polished followed by multiple annealing steps in reducing atmosphere with continuous flow of high purity hydrogen gas through the furnace chamber and then furnace cooled with the last stage in high purity Ar to reverse any hydrogen adsorption. After each processing step, 20 indentations were carried out within the same millimeter diameter grain, orientation $\langle 110\rangle$. The nanoindentation experiments were performed using a diamond Berkovich indenter tip and the Nano Flip (Nanomechanics Inc, Oak

Ridge, TN). The loading rate, $\dot{P}$, was controlled such that $\frac{\dot{P}}{P}$ was held constant at $0.21 / \mathrm{s}$. The maximum load was $24 \mathrm{mN}$. Justified by Fe's high ratio of elastic modulus to yield strength, the contact area was taken to be in the original plane of the surface (Fig 2.1b). This definition allows the hardness to be calculated as a continuous function of depth without utilizing dynamic testing methods that can obfuscate experimental data at small indentation depths. Electron backscatter diffraction (EBSD) experiments were performed to support the hypotheses derived from the nanoindentation experiments. These EBSD results were obtained using a FEI XL40 ESEM interfaced with the Oxford EBSD system.

\subsection{RESULTS AND DISCUSSION}

It is observed from the load-depth (P-h) curves in Fig. 2.1 and averaged hardness vs depth $(\mathrm{H}-\mathrm{h})$ profile plots in figure 2 that the hardness for the as polished sample is significantly greater than that for the partially or fully annealed samples. This is expected on the basis of FD strengthening (work hardening) of the surface due to mechanical polishing [10]. The degree of the indentation size effect (ISE) can be determined by noting the decrease in hardness with depth. As seen in figure 2.2, the ISE is most obvious for the un-annealed, mechanically polished specimen. 


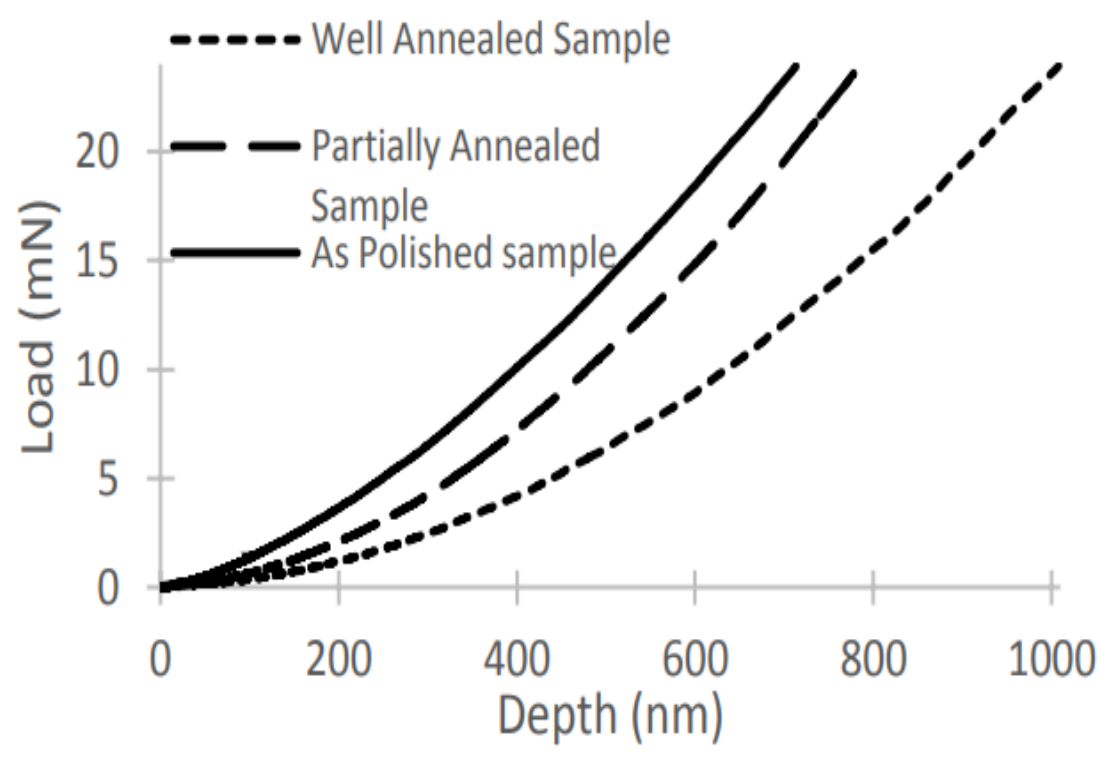

(a)

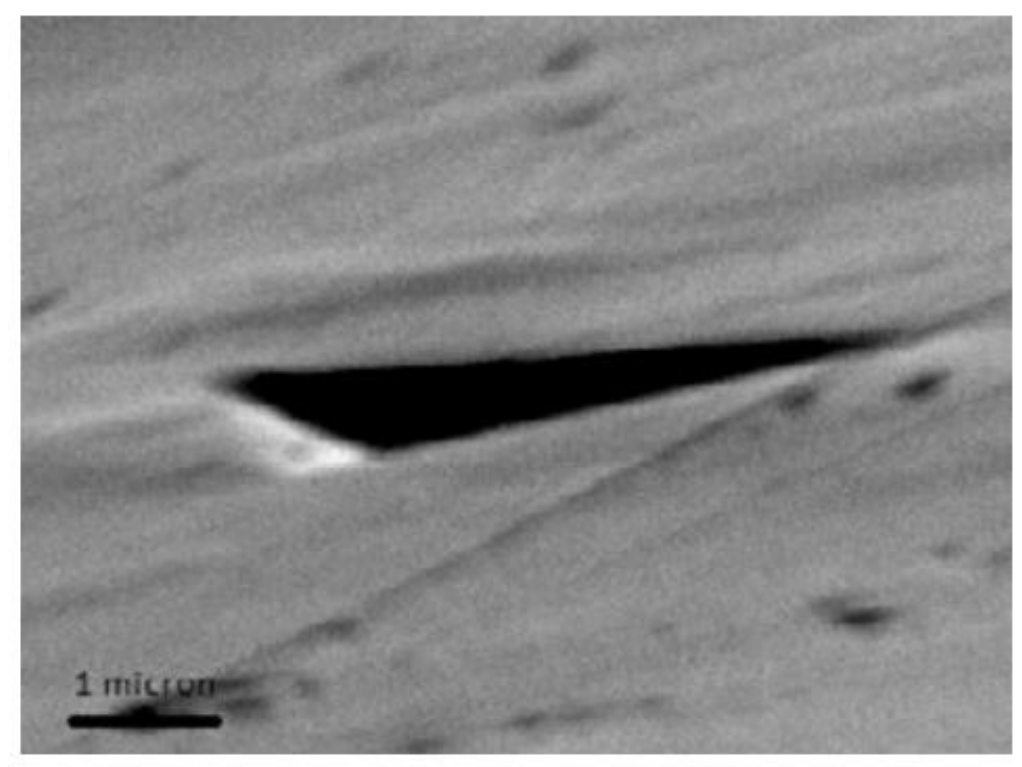

(b)

Figure 2.1. (a) Representative P-h curves, (b) SEM image of an indentation, at $\sim 80^{\circ}$ tilt. 


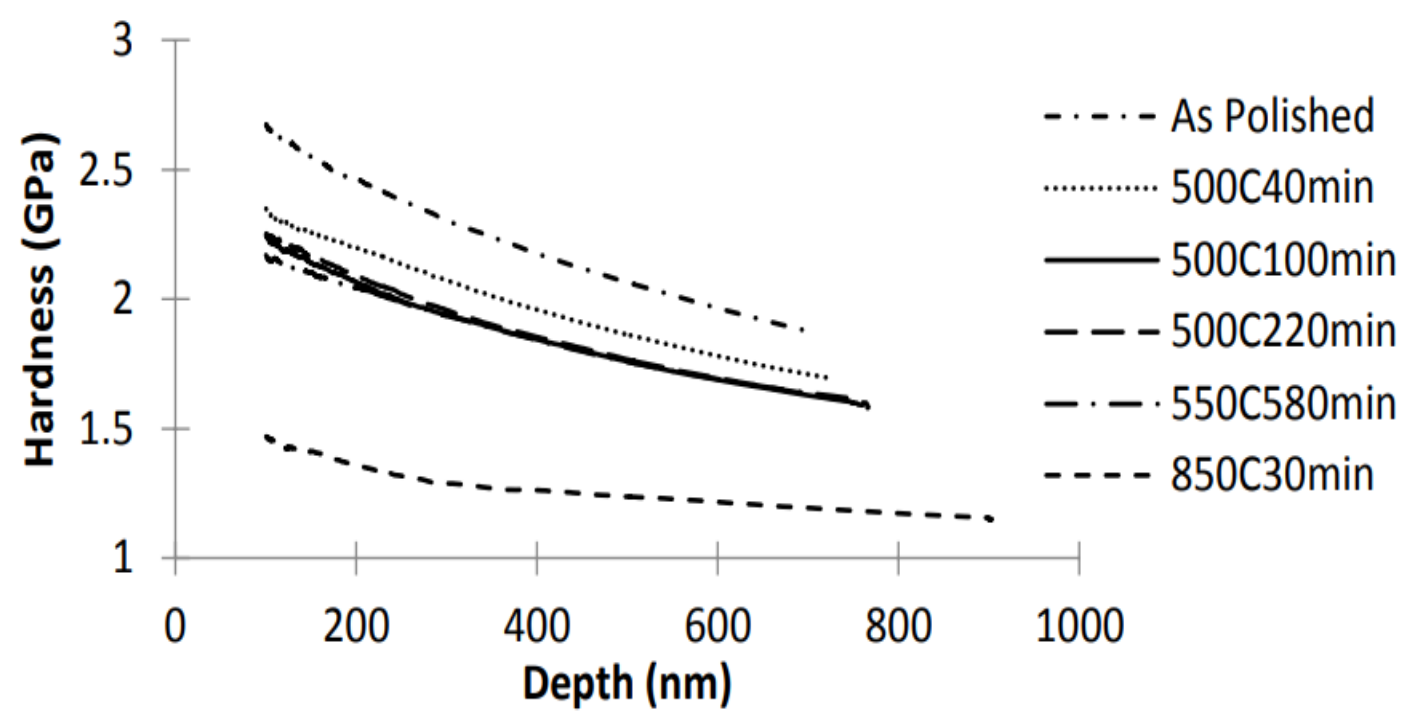

Figure 2.2. Plot between hardness and depth (averaged from $20 \mathrm{H}$ vs h curves for each condition) for all the processing conditions, indicating presence of indentation size effect (ISE).

The data in figure 2.2 is converted to Nix-Gao curves $\left(\mathrm{H}^{2}\right.$ vs $\left.1 / \mathrm{h}\right)$ in figure 2.3. According to the Nix Gao model, $\mathrm{H}^{2}$ vs $1 / \mathrm{h}$ plot should be linear, with the slope corresponding to the decrease in GND density with depth. Only the $850^{\circ} \mathrm{C}$ anneal sample approaches linear behavior. A qualitative inspection of figure 2.3 shows that the nonlinear (bilinear) behavior of the Nix-Gao plots is reduced when the sample is annealed, and the hardness is reduced. The reduction in hardness due to annealing is expected as the FD density is decreased during the annealing process. Adding the hardening effect of the FD density, $\rho_{F D}$, to the indentation GND and SSD density in equation (2.1) leads to:

$$
H^{2}=H_{o}^{2}\left(1+\frac{\rho_{F D}}{\rho_{S S D}}+\frac{h^{*}}{h}\right)
$$

A simple model for the reduction in FD density, $\rho_{F D}$, during the annealing recovery process has been proposed by Nes [11], written in the form which exposes the diffusion coefficient as 


$$
\sqrt{\rho_{F D}(t)}=\sqrt{\rho_{F D}(0) *\left(-\left[\frac{1}{A}\right]\left[\ln \left\{\left(\frac{1}{L^{2}}\right)\left[L^{2} \exp (-A)+D t\right]\right\}\right]\right)}
$$

Where $\mathrm{D}$ is the self-diffusion coefficient, $\mathrm{t}$ is annealing time, $\mathrm{Q}$ is the activation energy for self-diffusion, $L^{2}=\frac{b A}{\sqrt{\rho_{F D}(0)}}, A \sim \frac{G b^{3}}{K T}$.

Substituting equation 2.3 into equation 2.2 and differentiating with respect to $1 / \mathrm{h}$ suggests the behavior of the Nix Gao slope for a 1/h dependent polishing induced forest dislocation distribution as

$$
\frac{\partial}{\partial\left(\frac{1}{h}\right)} H^{2}=\frac{H_{o}^{2}}{\rho_{S S D}}\left[\frac{\partial}{\partial\left(\frac{1}{h}\right)} \rho_{F D}(0)\right]\left[\frac{\rho_{F D}(t)}{\rho_{F D}(0)}\right]+\left[\frac{\partial}{\partial\left(\frac{1}{h}\right)} \frac{\rho_{F D}(t)}{\rho_{F D}(0)}\right]\left[\rho_{F D}(0)\right]+H_{o}^{2} h^{*}
$$

Equation 2.4 considered together with equation 2.3 indicates the possibility that the Nix Gao slope obtained by indentation within a deformed, annealed surface layer can be a function of the product (Dt). The curves in figure 2.3 are fitted to $3 \mathrm{rd}$ order polynomials

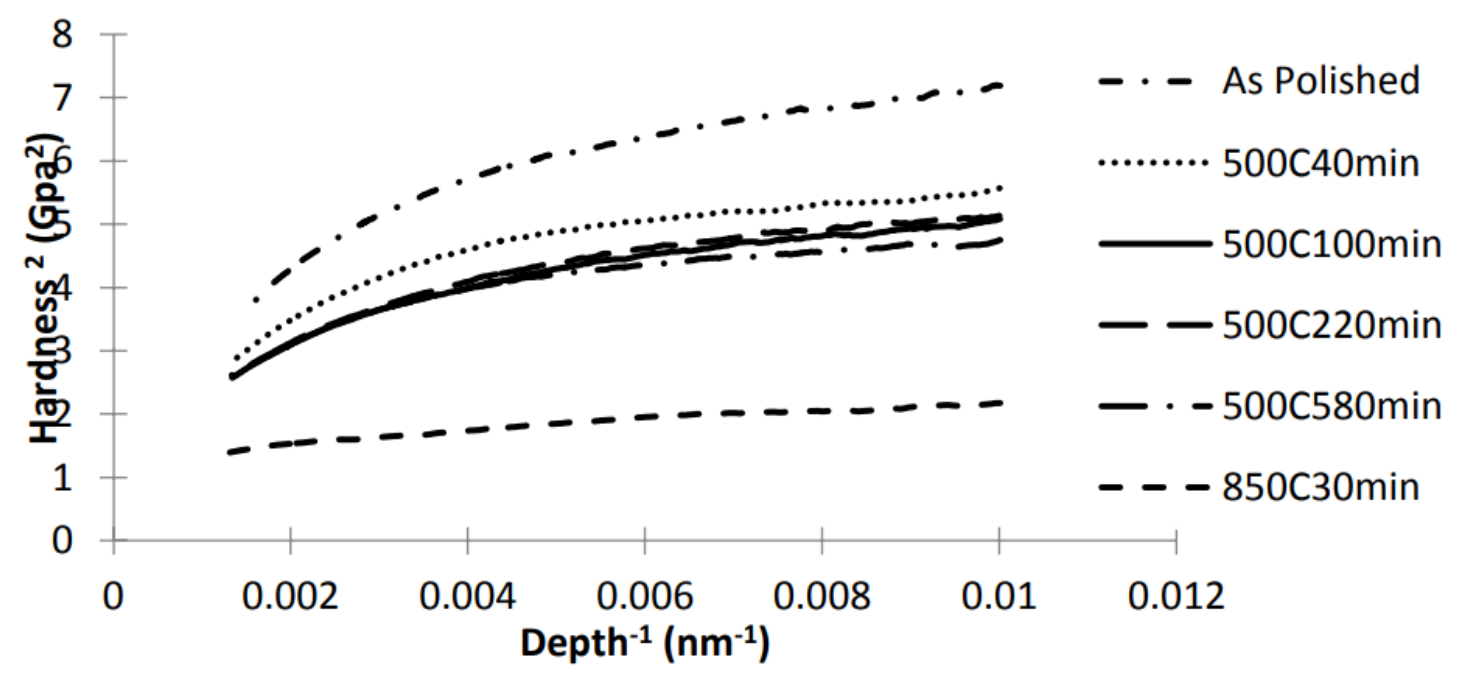

Figure 2.3. Nix-Gao plots for various processing conditions. 
(R2 >0.99) allowing the Nix Gao slope at $1 /(150 \mathrm{~nm})$ and $1 /(500 \mathrm{~nm})$ to be determined via derivative. A plot of the Hardness vs $\log \left(D t^{2}+L\right)$ and the (Nix-Gao slope) against $\log \left(D t^{2}+L\right)$ at $150 \mathrm{~nm}$ indentation depth and $500 \mathrm{~nm}$ indentation depth is shown in figure 2.4. The value of $\mathrm{L}^{2}$ for the $150 \mathrm{~nm}$ indentation depth is $10^{-19} \mathrm{~m}^{2}$ while the value of $\mathrm{L}^{2}$ for the $500 \mathrm{~nm}$ indentation depth is $10^{-18} \mathrm{~m}^{2}$. The result in figure $2.4 \mathrm{a}$ reproduces the logarithmic decay in hardness value for increasing Dt often observed for the dislocation recovery process on annealing, as reviewed in [11]

Figure $4 \mathrm{~b}$ shows the Nix Gao slopes at the $150 \mathrm{~nm}$ and $500 \mathrm{~nm}$ indentation depths approach one another as annealing Dt increases and provides a quantitative measure of how the bilinear behavior as the polishing surface damage is removed with increasing Dt. To the authors' knowledge, figure 2.4 is the first quantitative correlation between Nix-Gao bilinear behavior and the cold worked condition via mechanical polishing of the sample surface layer. However, it is noted that previous work has observed the Nix Gao plot for mechanically polished $\mathrm{Ni}$ has a slope in the $400-800 \mathrm{~nm}$ indentation depth range much larger than the Nix Gao plot for mechanically polished Ni that has been electro-polished or annealed [12]. It is also noted that indentation studies on $10 \%$ pre-strained $\mathrm{Cu}$ compared with well-annealed $\mathrm{Cu}$ [13] do not report the effect observed here and in [12], perhaps because of a more homogeneous distribution of forest dislocations in [13].

To extend this analysis further, we note the Nix-Gao theory can be used to rationalize the result in figure 2.4 with very little modification by invoking a depth dependence in the forest dislocation density induced by mechanical polishing and subsequently altered by annealing. Assuming equation (2.2) is 'correct' in the sense that the Nix Gao theory in equation (2.1) requires no modification other than the addition of the depth dependent FD density, the depth dependence of the FD density may be calculated, where $\mathrm{Ho}=1.15 \mathrm{Gpa}$ and $\mathrm{h}^{*}=70.58 \mathrm{~nm}$, are determined from the linear behavior of the $850^{\circ} \mathrm{C}$ annealed material. The result is plotted in figure 2.5. 


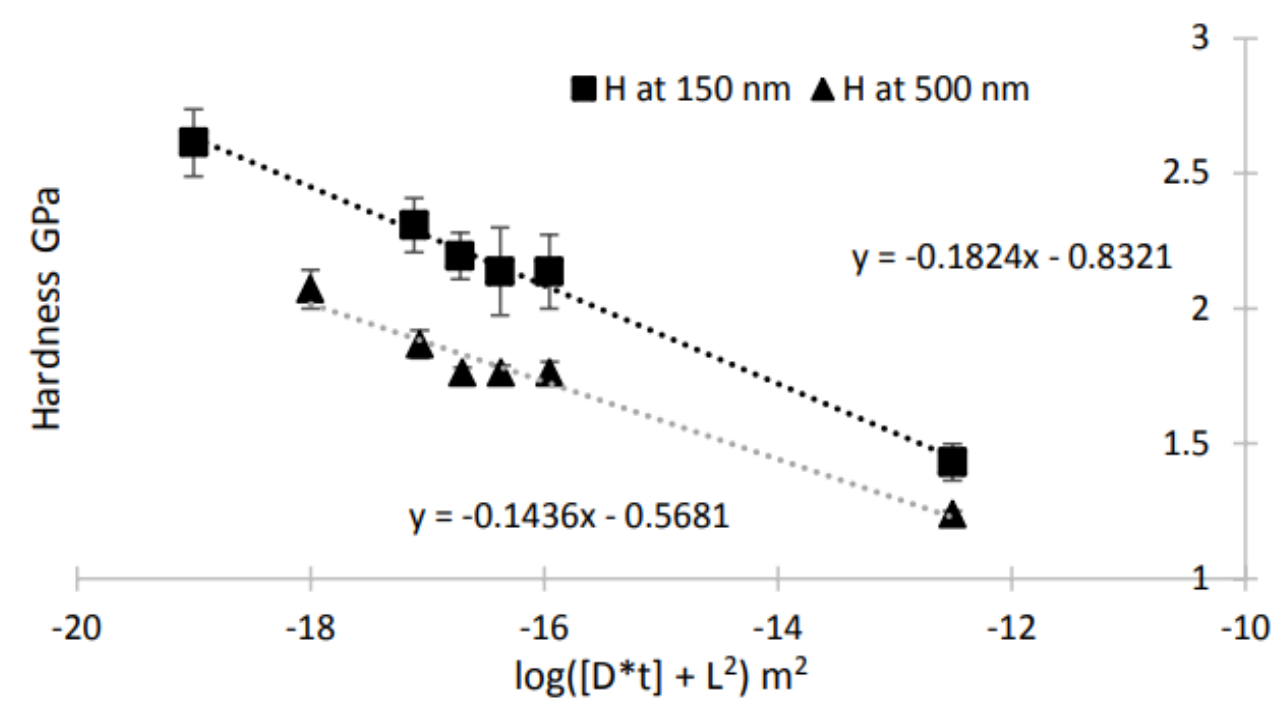

(a)

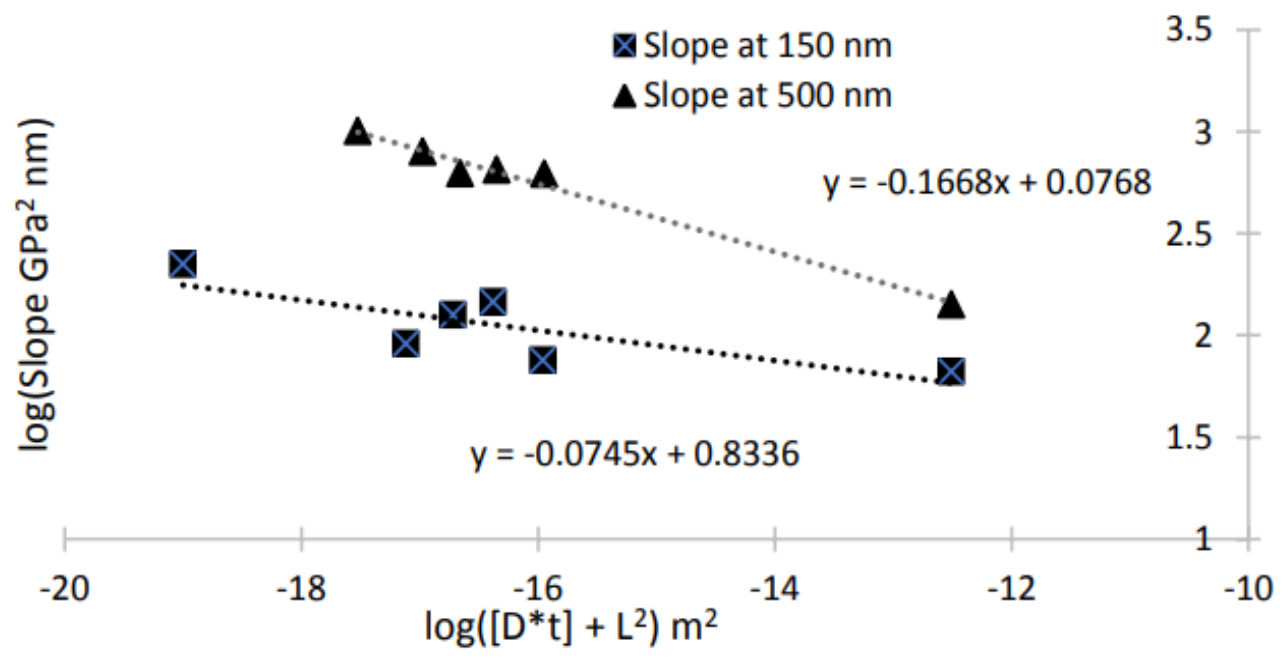

(b)

Figure 2.4. (a) The hardness - Log(diffusion length) plot of hardness measured at $150 \mathrm{~nm}$ and 500nm showing a typical logarithmic decay in flow stress with heat treat recovery time, (b) The reduction in bilinear behavior with annealing of surface polishing deformation is indicated as the $150 \mathrm{~nm}$ and 500nm slopes approach one another. 
The concept that the observed large changes in hardness and Nix Gao slope at low indentation depth (Fig. 2.4) are correlated with annealing induced changes in $\rho_{\mathrm{FD}}$ (Fig. 2.5)

is qualitatively tested using EBSD Kernel Average Misorientation (KAM) and Pattern

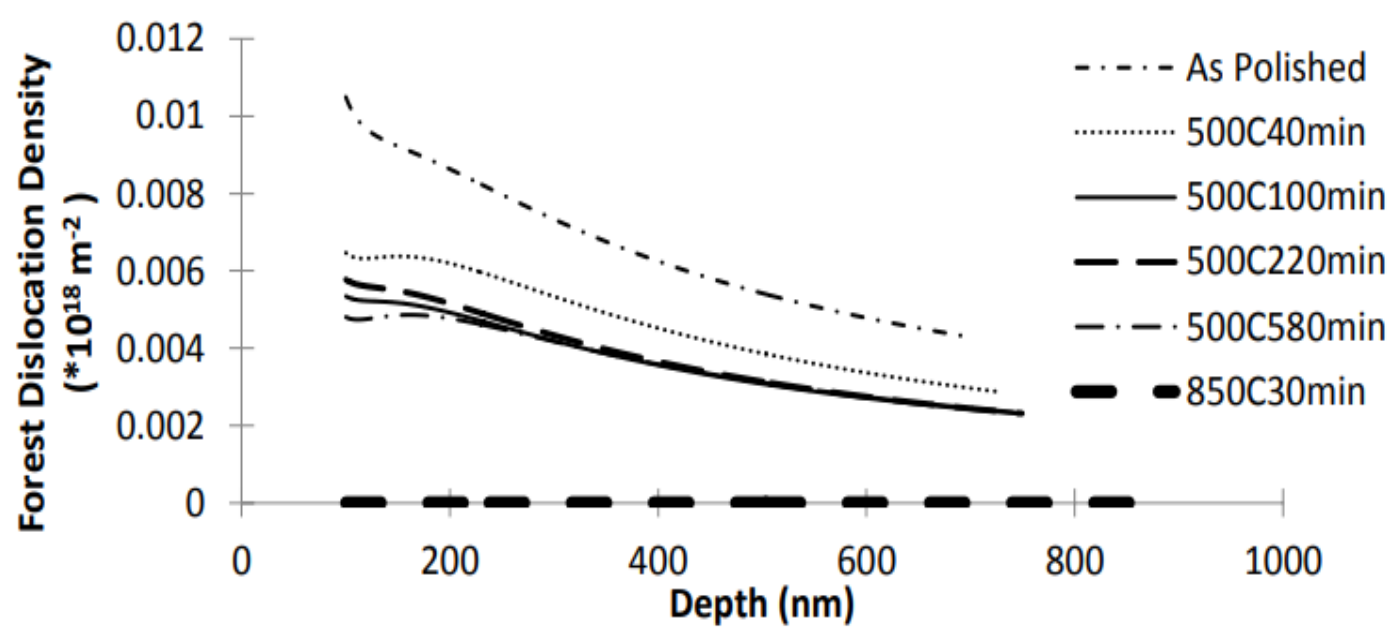

Figure (2.5): Plot of calculated forest dislocation density as a function of depth. The density is calculated assuming linear Nix-Gao ISE for the various processing conditions.

Quality (PQ) measurements of surface layer deformation [14-15]. Although PQ is generally considered a qualitative measure of surface deformation, we do consider an adaptation of the pattern quality metric of [15], where the parameter proportional to the stored deformation energy (SFD) introduced by mechanical polishing is given as

$$
S_{F D}=\left[1-\frac{Q_{F D(\mathrm{av})}-Q_{F D(\min )}}{Q_{F D(\max )}-Q_{F D(\min )}}\right]
$$


The $\mathrm{Q}$ in equation 2.5 are the average, minimum and maximum pattern quality values for the distribution in the deformed (via polishing) or deformed (via polishing) and annealed samples.

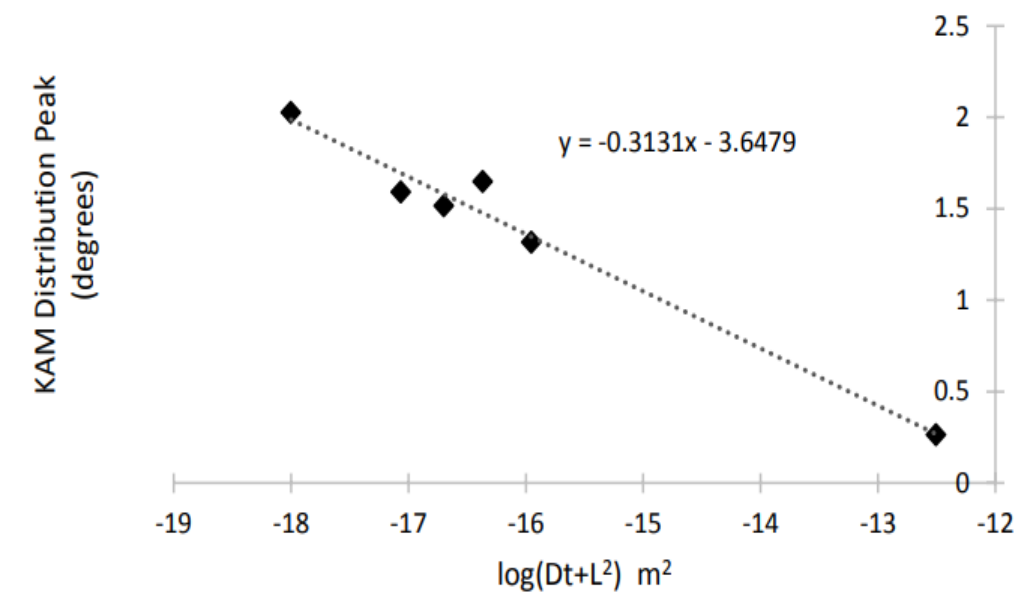

(a)

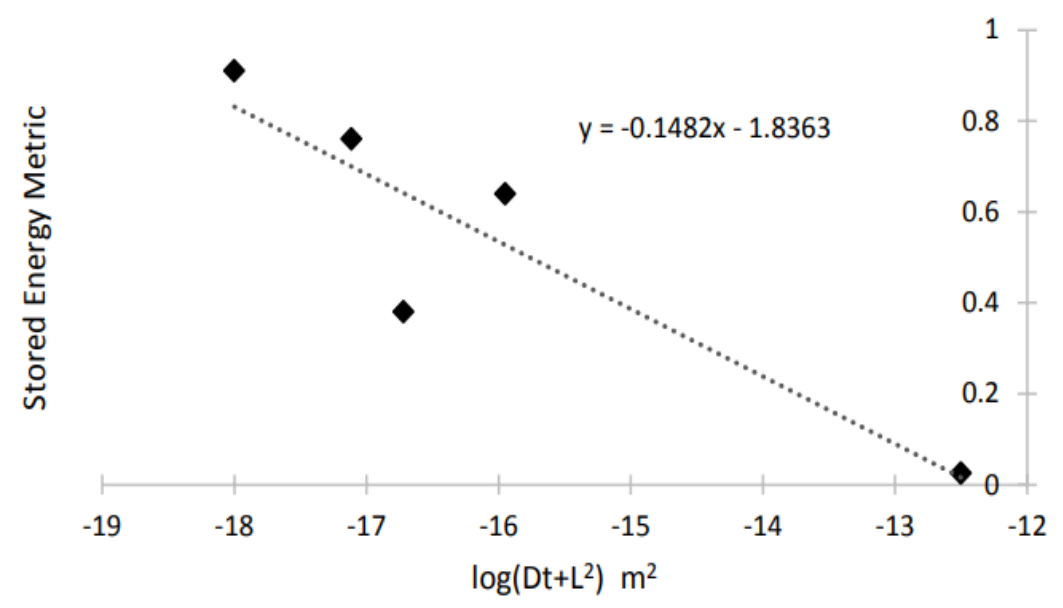

(b)

Figure 2.6. (a) KAM, and (b) PQ metrics plotted against the logarithm of annealing duration. 
The uncorrelated average KAM value [14] and a pattern quality metric [15] are plotted against the logarithm of the annealing time figure 2.6. Both representations in figure 2.6 support the argument of the paper, with the caveat that these surface deformation measurements by EBSD are from 20-50 $\mathrm{nm}$ in depth while the indenter tip is sensing material behavior well below $150 \mathrm{~nm}$.

\subsection{CONCLUSIONS}

The effect of processing conditions on indentation behavior, particularly on indentation size effect, were studied for high purity $\alpha$-Fe. The transition from bilinear NixGao behavior exhibited by cold worked (by mechanical polishing) and partially

annealed samples to the predicted characteristic linear behavior for a well annealed surface has been correlated to the annealing diffusion length. Moreover, it is possible to recover the linear Nix Gao model from bilinear data if the forest dislocation density due to surface polishing is considered a function of indentation depth.

\subsection{ACKNOWLEDGMENTS}

This work was supported by Office of Basic Energy Sciences within the Department of Energy (DOE) Office of Science: Award Number: DE-SC0016314 


\section{REFERENCES}

1. T. T. Zhu, A. J. Bushby, Dunstan, Mater. Tech. 23, 193-209 (2008)

2. K. W. McElhaney, J. J. Vlassak, W. D. Nix, J. Mater. Res. 13, 1300-1306 (1998)

3. Q. Ma, D. R. Clarke, J. Mater. Res. 10, 853-863 (1995)

4. N. A. Fleck, G. M. Muller, M. F. Ashby, J. W. Hutchinson, Acta. Metall. Mater. 42, 475-87 (1994)

5. M. E. Gurtin, J. Mech. Phys. Solids 50, 5-32 (2002)

6. W. D. Nix, H. Gao, J. Mech. Phys. Solids 46, 411-425 (1998)

7. L. P. Evers, D. M. Parks, W. A. M. Brekelmans, M. G. D. Geers, J. Mech. Phys. Solids 50, 2403-2424 (2002)

8. G. M. Pharr, E. G. Herbert, Y. Gao., Annu. Rev. Mater. Res. 40, 271-292 (2010)

9. F. K. Durst, B. Backes, M. Goken, Scr. Mater. 52, 1093-1097 (2005)

10. L. E. Samuels, Journal of the Institute of Metals. 85, 51-62 (1956)

11. E. Nes, Acta Metallurgica et Materialia 43, 6, 2189-2207 (1995)

12. W. Zhigang, PhD Thesis, University of Tennessee, 2012

13. B. Backes, Y. Huang, M. Goken, K. Durst, J.Mat.Res., 24, 8, 1197-1207 (2008)

14. L. N. Brewer, D. P. Field, C. C. Merriman (2009) Springer, Mapping and Assessing Plastic Deformation Using EBSD. In: Schwartz A., Kumar M., Adams B., Field D. (eds) Electron Backscatter Diffraction in Materials Science. Springer, Boston, MA 15. A.J. Wilkinson, D.J. Dingly, Acta Metal. Mater. 39, 3047 (1991) 


\section{Appendix 2.A Reprint Copywrite Permission}

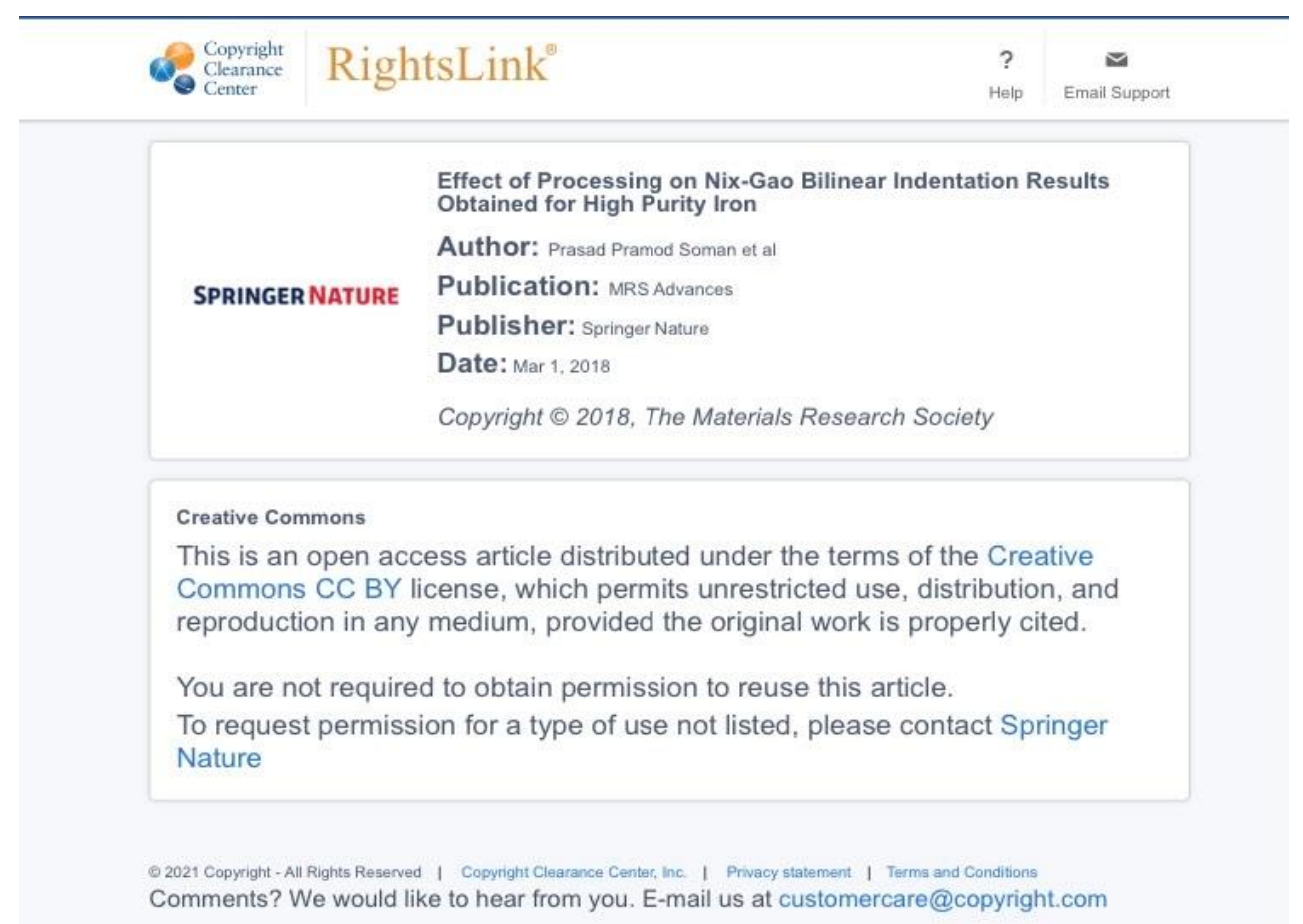




\section{CHAPTER 3: ANALYSIS OF LOCAL GRAIN BOUNDARY STREGTHENING UTILIZING THE EXTRINSIC INDENTATION SIZE EFFECT}

Published in Journal of Materials Research - DOI: 10.1557/jmr.2019.102

\subsection{ABSTRACT}

The extrinsic indentation size effect (ISE) is utilized to analyze the depth-dependent hardness for Berkovich indentation of non-uniform dislocation distributions with one- and two-dimensional deformation gradients and is then extended to indentation results at grain boundaries. The role of the Berkovich pyramid orientation and placement relative to the grain boundary on extrinsic ISE is considered in terms of slip transmission at yield and plastic incompatibility during post-yield deformation. The results are interpreted using a local dislocation hardening mechanism originally proposed by Ashby, combined with the Hall-Petch equation. The Hall-Petch coefficient determined from the extrinsic ISE of the grain boundary is found to be consistent with the published values for pure Fe and mild steel. A simple, linear continuum strain gradient plasticity model is used to further analyze the results to include contributions from a non-uniform distribution in plastic strain and dislocation density.

\subsection{INTRODUCTION}

The indentation size effect (ISE) has been studied for two decades, with the most accepted model of the physical origin presented by Nix and Gao [1]. In the context of examining the ISE in thin films, the terms "intrinsic" and "extrinsic" size effect were introduced by $\mathrm{Ngan}$ and $\mathrm{Ng}$ [2] to distinguish between the indentation response of the film itself versus the combined response of the film/substrate system. Following a slightly modified form of that nomenclature, here we compare and contrast the intrinsic and 
extrinsic ISE in high-purity and carbon-doped iron. In this context, we use the terms intrinsic and extrinsic to distinguish between the effect of the indenter geometry-induced plastic strain gradient [1], as observed in well-annealed, single crystal surfaces, and the effect(s) of preexisting defect structures in the same material, respectively. Based on that distinction, the depth-dependent hardness, $\mathrm{H}$, as measured by Berkovich nanoindentation experiments, is used to interrogate the structure-property relationship of specific defect structures that show extrinsic size effects. The four cases analyzed are (i) three different well-annealed, single-crystal Fe surfaces (intrinsic ISE), (ii) a deformed surface layer for one of the same grains, (iii) a "cylinder" of plastic deformation produced by a linear array of shallow indents for the same grain, and (iv) four random grain boundaries of varying tilt/ twist character. This paper does not attempt to address the fundamental mechanisms responsible for the details of the intrinsic ISE in well-annealed, low-dislocation density materials. Instead, the paper examines how the depth-dependent $\mathrm{H}$ may be correlated with the configuration and strengthening properties of preexisting defects.

The analysis implemented here adopts the mathematically transparent, mechanistic model of Nix and Gao [1] for ISE, which is based on the widely accepted concept of Taylor hardening. The analysis is then extended with a strain gradient plasticity (SGP) model, which includes spatial dependence of plastic strain. In the Nix-Gao model [1], the Taylor hardening concept was combined with the concept of geometrically necessary dislocations (GNDs) [3] that would be required to accommodate the gradient in plastic strain imposed by a geometrically self-similar indenter tip such as the Berkovich. The GNDs were considered as additive to the statistically stored dislocations (SSD) in the Taylor expression, where the SSD are due to the indentation characteristic strain. Both the SSD and GND densities were considered as being uniform (averaged) throughout the indentation volume [1]. To explain decreasing hardness with increasing depth, this model proposes that GND have an increase in spacing as the indentation depth increases, causing a reduction in Taylor hardening. The Nix-Gao model for indentation of initially defect- 
free material relates the hardness, $\mathrm{H}$, to the indentation-induced averaged dislocation density as,

$$
\begin{gathered}
H=H_{0} * \sqrt{1+\frac{h^{*}}{f^{3} h}} \\
H_{O}=3 \sqrt{3} \alpha G b \sqrt{\rho_{S S D}} \\
h^{*}=\frac{3 \tan ^{2} \theta f^{2}}{2 b \rho_{S S D}} \\
\rho_{G N D}=\frac{3 \tan ^{2} \theta}{2 f b h}
\end{gathered}
$$

where $\mathrm{H}_{\mathrm{o}}$ is the bulk hardness free of GNDs; $h^{*}$ is the characteristic depth; $\mathrm{f}$ is a material-dependent parameter introduced by Durst et al. [4], which attempts to provide a more accurate estimate of the hemispherical volume containing the GNDs; $a$ is a constant taken to be $0.5, \mathrm{G}$ is the shear modulus; $\mathrm{b}$ is the Burgers vector; $\rho_{S S D}$ and $\rho_{G N D}$ are the SSD and GND densities, respectively; and $h$ is the angle between the indenter and the undeformed surface.

Literature describing how the ISE could be used to determine the defect configuration and/or structure-property relationships in the presence of preexisting defects is relatively rare. Specific examples taking this approach can be found in the grain boundary indentation literature and in the analysis of thin film indentation $[2,5,6]$. In a manner that is directly related, changes in the ISE due to subsurface dislocation gradients created by mechanical polishing have been examined in high-purity Ni [7]. In this case, over indentation depths from 400 to $800 \mathrm{~nm}$, it was shown that a plot of $\mathrm{H}^{2}$ versus $1 / \mathrm{h}$ for mechanically polished $\mathrm{Ni}$ has a larger slope, $\alpha . h^{*}$, than the equivalent plot for mechanically polished Ni that has been subsequently electropolished [6]. Similarly, as suggested by Pharr et al. [8], differences in the measured $\mathrm{h}^{*}$ of pure $\mathrm{Cu}$ between different research groups 
$[9,10]$ can be attributed to variations in the ISE due to the effect of mechanical polishing. However, attempts to quantitatively relate this variance in the ISE to the defect configuration within the stressed volume have not been made. Examination of the ISE in work-hardened $\mathrm{Cu}$ (uniform macroscopic strain) versus well-annealed $\mathrm{Cu}$ was studied by Poole et al. [11], where it was shown that cold work does not affect the slope of the $\mathrm{H}-\mathrm{h}$ curve but does increase the magnitude of $\mathrm{H}$. As a counterexample to Poole et al. [11], Backes et al. [12] reported a reduction in the ISE as measured in $10 \%$ prestrained $\mathrm{Cu}$ compared with well-annealed $\mathrm{Cu}$. In contrast to the work by Poole et al. [11] and Backes et al. [12], where the deformation is relatively homogeneous, the variance in ISE reported in Refs. 7, 10, and 13 and reported herein is attributed to gradients in the subsurface damage and, thus, is considered a direct result of the inhomogeneity in the preexisting forest dislocation (FD) density near the free surface.

Despite a rapidly expanding pool of literature on grain boundary indentation and the ISE, it does not appear the two have been studied or analyzed in the manner presented here $[5,6,14,15]$. Specifically, it is found that most published Berkovich indentation studies have focused on "remote" indentation, as shown in Fig. 3.1(a), where the indenter tip is a significant distance from the grain boundary and, thus, the $\mathrm{H}$ variation with $\mathrm{h}$ is studied as the plastic zone approaches the grain boundary. Remote indentation with the face of the pyramidal indenter parallel to the grain boundary would seem to accentuate analysis of slip transmission across the boundary, as the angle of the indenter face is most acute here and the rate of approach of the indenter face with increasing $h$ is minimized. In the analysis presented here, the goal is to place the pyramidal Berkovich indenter tip as close to the grain boundary as possible. Due to constraints imposed by the optical microscope and screw-driven stages, the targeting accuracy is $\sim \pm 1 \mu \mathrm{m}$. Within this range, deviations in the precise placement of the indenter tip are deemed acceptable, provided the tip is oriented such that the wedge of the pyramid formed by the intersection of the pyramid faces is approximately perpendicular to the grain boundary plane trace, as shown in Fig. 3.1(b). Due to the obtuse angle of the indenter geometry at the face intersections, this 
orientation effectively places a wedge across the grain boundary plane, inducing nearly simultaneous initiation of deformation in the adjacent grain at very small indentation depths. This contact orientation produces $\mathrm{H}-\mathrm{h}$ curves, which typically do not show a discontinuity (pop-in) at indentation depths $>100 \mathrm{~nm}$, as compared with those

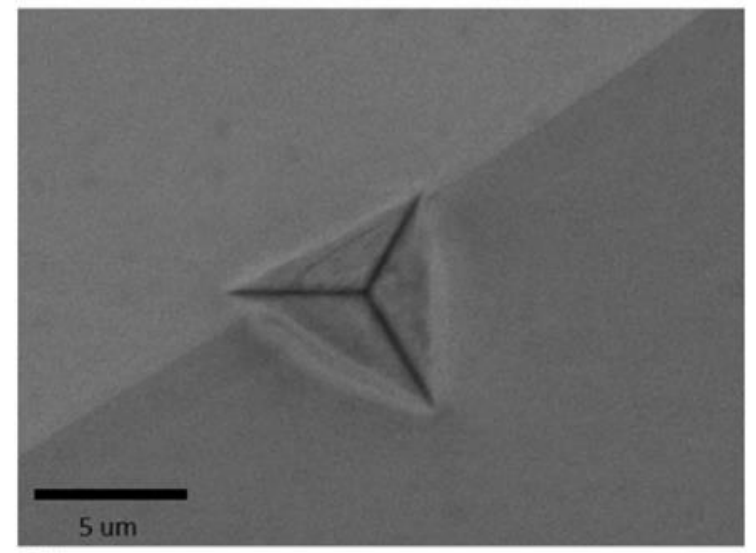

(a)

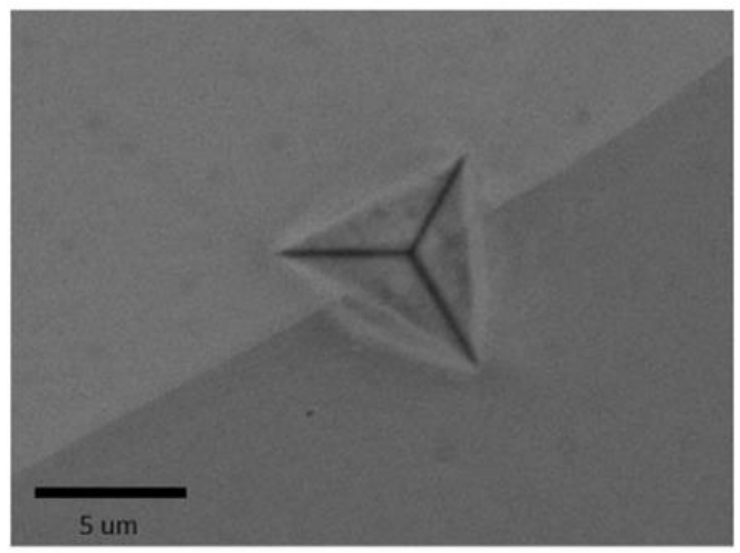

(b)

Figure 3.1. Backscatter channeling contrast image of (a) Remote grain boundary indentation: the indenter tip is positioned $\sim 2$ micron from the grain boundary and the flat face of the indenter parallel to the boundary, (b) Local grain boundary indentation: the 
indenter tip is positioned less than 0.5 micron from the grain boundary with the "wedge" of indenter being perpendicular to the grain boundary plane.

shown in Refs. 16, 17, and 18. However, the $\mathrm{H}-\mathrm{h}$ behavior in this orientation does show an increase in $\mathrm{H}$ above that of both adjacent grain interiors and a unique extrinsic ISE, again quite distinguishable from the intrinsic ISE for either of the adjacent grains.

It is proposed that the indentation geometry primarily utilized here [Fig. 3.1(b)] would tend to emphasize a grain boundary strengthening effect that occurs at plastic strain significantly beyond the yield point, as originally envisioned by Ashby [3] and correlated with the concept of plastic strain anisotropy across the grain boundary [19]. It is noted that there has been some work in the literature relating nanoindentation results to the Ashby proposal of plastic strain incompatibility, although not utilizing the same approach as in this paper. Vachhani et al. [20] used spherical nanoindentation near grain boundaries of deformed polycrystalline $\mathrm{Al}$ to examine local hardening at the grain boundaries due to prior macroscopic deformation. Vachhani et al. [20] correlated this local hardening to plastic anisotropy through the Taylor factor mismatch across the boundary. It was not the focus of the authors [20] in this previous work to examine grain boundary indentation in wellannealed samples or any variance in the ISE between indentation within a well-annealed grain interior and indentation at a well-annealed grain boundary, as in the current paper, but rather to expose the localized hardening at the grain boundary due to macroscopic deformation. Nevertheless, the Vachhani et al. [20] findings are important to the interpretation presented in this paper.

As this paper utilizes the Nix-Gao [1] approach to interpret the extrinsic ISE due to grain boundaries and non-uniform dislocation distributions, it is useful to examine other works that are related to this approach. The works presented by Hou et al. [5] and Jung et al. [15] have the most in common with the ISE analysis presented here. Of particular interest, Jung et al. utilized a modified Nix-Gao relationship [Eq. 3.1(a) - 3.1(d)] to analyze the ISE contribution of grain boundaries but examined the remote indentation results of 
Yang et al. [21] obtained from the center of $\mathrm{Ni}$ grains rather than local to the grain boundary. Another relevant grain boundary investigation is that of Xiao et al. [22], wherein the remote indentation strengthening effect is also analyzed using the Nix-Gao relationship. Xiao et al. proposed that the grain boundary effectively reduces the volume of the plastic zone without affecting the number or length of the intrinsic GNDs, thus increasing the dislocation density that results in Taylor hardening [22]. Although the approach of Xiao et al. is similar to the treatment here, the analysis is limited to slip transmission induced by remote indentation [Fig. 3.1(a)] and does not specifically analyze "local" indentation [Fig. 3.1(b)] and plastic incompatibility at the grain boundary. In contrast to these investigations, we propose that aspects of the originality of the experimental observations presented here are associated with the orientation, asymmetry, and location of the Berkovich indentation with respect to the grain boundary plane. As such, our analysis of experimental observations focuses on the abrupt imposition of equivalent stress above the yield stress associated with Berkovich indentation at the grain boundary rather than the gradual increase in stress with increasing $\mathrm{h}$ at the boundary plane related to remote Berkovich indentation or local spherical indentation [23].

Previously, we proposed that changes in the slope of $\mathrm{H}^{2}$ versus $1 / \mathrm{h}$ can be utilized to study an FD density gradient induced by mechanical polishing through direct comparisons to the $\mathrm{H}$ measured in the same but well-annealed grain [13]. To support the proposed concept of using the extrinsic ISE to examine the relevant microstructures, here we first present new results that reinforce our previous findings. Examination of this phenomenon leads to the rather simple, but previously unexplored, concept that the averaged spatial variation of the FD density (due to mechanical polishing) with depth can contribute to the nonlinear $\mathrm{H}^{2}$ versus $1 / \mathrm{h}$ behavior. The paper then extends this defectrelated extrinsic ISE to the analysis of a cylinder of plastic deformation produced by a row of shallow indents. These two cases of local defect analysis are then compared with grain boundary mechanical behavior as exposed by an ISE different from the intrinsic ISE of the adjacent grain interiors. 
To interpret the ISE and capture the effect of GNDs, a continuum SGP model is employed. Such frameworks, initially proposed by Aifantis [24] consider the gradient of the plastic strain as an independent variable and demonstrated by Fleck et al. [25] and Aifantis [26] are able to capture the scale of the microstructure, allowing the interpretation of size effects. More recently, interface energy terms within gradient plasticity developed by Aifantis and Willis [27, 28] have been able to account for the existence of grain boundaries during nanoindentation experiments [16]. The present experiments are able to further capture the effect of grain boundaries and provide additional qualitative understanding of the mechanically induced interface energy terms, while at the same time probing further into the ISE effect.

\subsection{EXPERIMENTAL WORK}

Cylindrical disks of high-purity (99.999\%) Fe $10 \mathrm{~mm}$ in diameter and $4 \mathrm{~mm}$ thick were annealed for over $40 \mathrm{~h}$ at $800{ }^{\circ} \mathrm{C}$ in a hydrogen atmosphere to create grain sizes up to $2 \mathrm{~mm}$ in diameter. The large grain size is necessary to obtain multiple indentations at a spacing of $>20 \mu \mathrm{m}$ along the grain boundary. Following the long-term anneal, samples were mechanically polished using grinding papers ranging from P800 to P-2400. Subsequently, the specimens were sequentially polished using $1,0.3$, and $0.05 \mu \mathrm{m}$ alumina slurries. Following the mechanical polishing, plasma etching was performed to remove any contaminants, such as, but not limited to, mineral deposits and organic residue, left behind from the mechanical polishing and washing process. The etching was performed using the Anatech Hummer 6 (Hayward, California). Following plasma etching, the specimens were heat treated in a hydrogen atmosphere at $850^{\circ} \mathrm{C}$ for $4-5 \mathrm{~h}$. Annealing at $62 \%$ of the absolute melting temperature, the goal was to eliminate any remaining gradient in the dislocation density relative to the free surface and remove any carbon-based contamination. After annealing, the specimens were cooled in an Ar atmosphere to ensure excess hydrogen was removed from the free surface, as the literature shows that hydrogen effects on Fe yield 
point [29] and hydrogen desorption from Fe dislocation and grain boundary "traps" occurs at baking temperatures less than $250^{\circ} \mathrm{C}[30]$.

After the $\mathrm{H}_{2}$ :Ar annealing, ion milling was performed using the JEOL IB-19500CP (Peabody, Massachusetts) cross-section polisher. Operating with a voltage of $6.5 \mathrm{kV}$ for 10 min followed by the fine mode milling, the low-energy argon beam was used to remove any contaminants inadvertently deposited on the surface during heat treatment. The assessment of the quality and structure of the free surface region in relation to this sample preparation process is considered a critical aspect of the experimental procedure for the nanoindentation study. Specimen surfaces were characterized using an Oxford electron backscatter diffraction (EBSD) system interfaced with the FEI XL40 environmental scanning electron microscope. EBSD images and kernel average misorientation (KAM) maps show the mechanically polishing, followed by plasma etching, annealing, and ion milling produces surfaces [Fig. 3.2(a) and 3.2(b)] with very low dislocation density compared with the unannealed mechanically polished KAM [Fig. 3.2(c) and 3.2(d)]. The step size used for the KAM analysis was $0.5 \mu \mathrm{m}$ with a filter size of $3 \times 3$ and a threshold angle of $5^{\circ}$.

Additional evidence to support this claim of low surface dislocation density is presented in Fig. 3.2(e), which shows shallow indentation depth pop-in behavior that is indicative of dislocation nucleation in the nearly dislocation-free surface. Note that Fig. 3.2(a) is a KAM map of Berkovich indentations along a grain boundary and thus indicates that the indentation contact area is much smaller than the grain size. The free surface and grain boundaries were studied in the annealed state, the deformed state, and also after being doped with carbon in the annealed state. Carbon doping allows the comparison of indentation results at the same grain boundary, with and without carbon. The carbon doping was carried out by encapsulating the high-purity Fe specimen discs and $0.01 \mathrm{mg}$ of polyethylene as a carbon source inside an evacuated quartz tube that was subsequently heat treated at $500^{\circ} \mathrm{C}$ for $3 \mathrm{~h}$. This heat treatment time and temperature give a carbon diffusion 
length of $\sim 3 \times 10^{4} \mathrm{~m}$ and a maximum average carbon concentration within the diffusion zone of $\sim 0.002$ weight percent. Using a focused beam of high-energy $(30 \mathrm{kV})$ gallium ions, the Hitachi FB-2000A (Krefeld, Germany) focused ion beam (FIB) mill was used to create FIB cross sections of residual hardness impressions [Fig. 3.3(a)] for analysis in the FEI (Einhoven, Netherlands) $200 \mathrm{kV}$ Titan Themis scanning transmission electron microscope (S-TEM). As shown in Fig. 3.3(b), the grain boundary is perpendicular to the free surface. The diffraction pattern represented in Fig. 3.3○ shows no evidence of additional phases such as $\mathrm{Fe}_{3} \mathrm{C}$ that could have formed during carbon doping.

Nanoindentation experiments were performed using a diamond Berkovich indenter tip and the iNano (Nanomechanics Inc.-KLA Tencor, Oak Ridge, Tennessee). The loading rate, $\dot{P}$, was controlled such that $\frac{\dot{P}}{P}$ was held constant at $0.21 / \mathrm{s}$. Analysis of the ISE was performed from experiments terminated at a maximum load of $24 \mathrm{mN}$. Rather than calculating the contact depth by subtracting the elastic deflection of the surface (as prescribed by the Oliver-Pharr model), here we take the contact depth, $\mathrm{h}_{\mathfrak{c}}$, to be in the original plane of the surface, i.e., $h_{c}=h$. As justification for this simplification, Fig. 3.2(f) shows a secondary electron SEM image of a representative residual hardness impression from an experiment performed to the maximum load used in this study of $24 \mathrm{mN}$. Indicative of well-annealed iron's significant capacity to work harden, the image shows no significant evidence of pileup, as the contact edges connecting the 3 corners are relatively straight, smooth lines that appear to be in the original plane of the surface. The unique, minor deviations in linearity observed along each of the 3 faces are taken to be the result of anisotropy effects. Upon confirmation of the microscope's $x-y$ calibration, the measured projected contact area is compared with the calculated contact area (outlined by the red boundary) and is found to be greater than the calculated area by $2.4 \%$. This small degree of error is consistent with the predictions of Lucas [31]. We note that since the ISE forces the loss of geometric self-similarity of the contact, the error imposed by the simplified definition may increase inversely with indentation depth. However, the simplified definition of the contact area is further justified for two reasons. First, this investigation 
focuses on the measured difference between the intrinsic and extrinsic size effect in alpha iron rather than the absolute value of the measured hardness. In this case, the error in the $\mathrm{h}_{\mathrm{c}}=\mathrm{h}$ approximation is associated with the difference in the elastic deflection between the intrinsic versus extrinsic ISE and not the absolute value of the elastic deflection. Second, due to the test specimen's high ratio of E/ $\sigma_{\mathrm{y}}(211 \mathrm{GPa} / 50 \mathrm{MPa}=4220)$, accurate measurements of the elastic contact stiffness as a continuous function of depth and, hence, accurate estimates of the elastic deflection of the surface are 


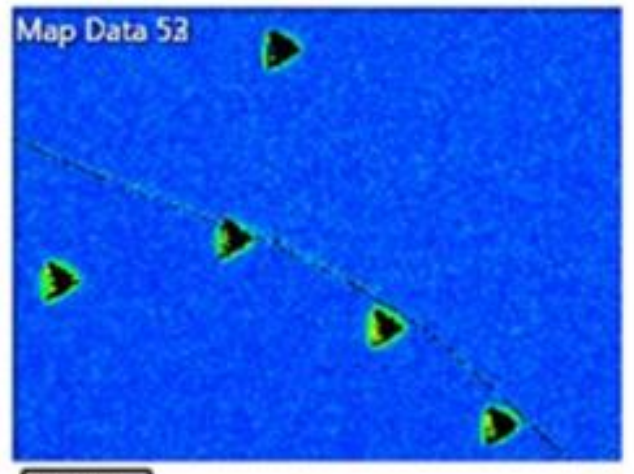

$25 \mu \mathrm{m}$

(a)

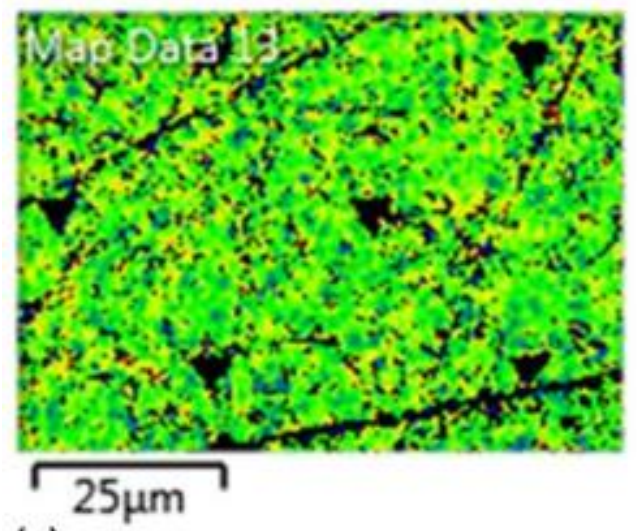

(c)

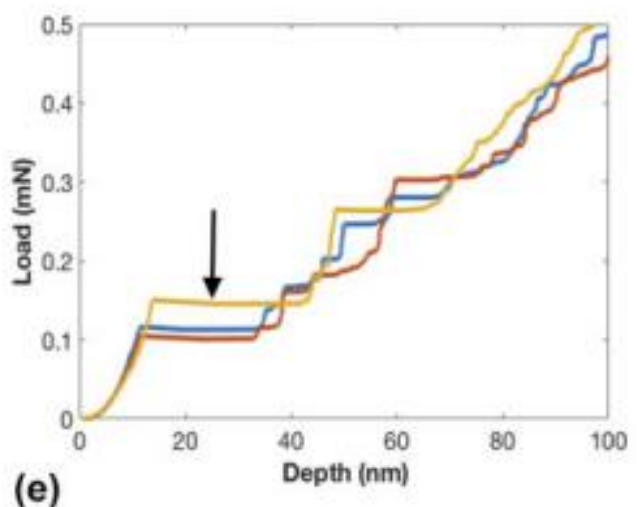

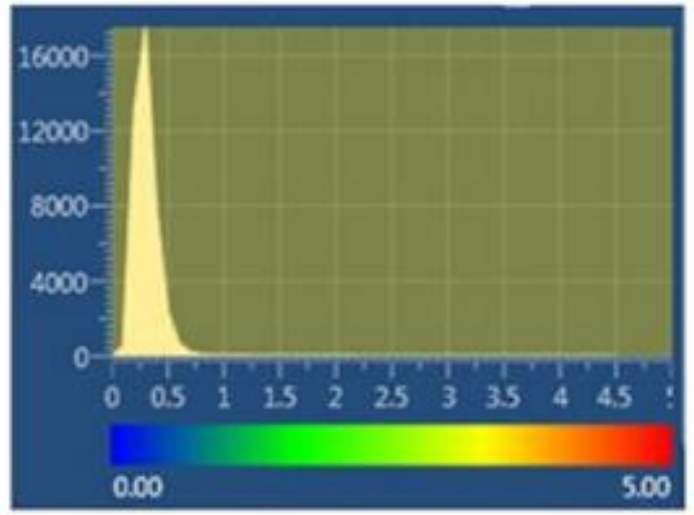

(b)

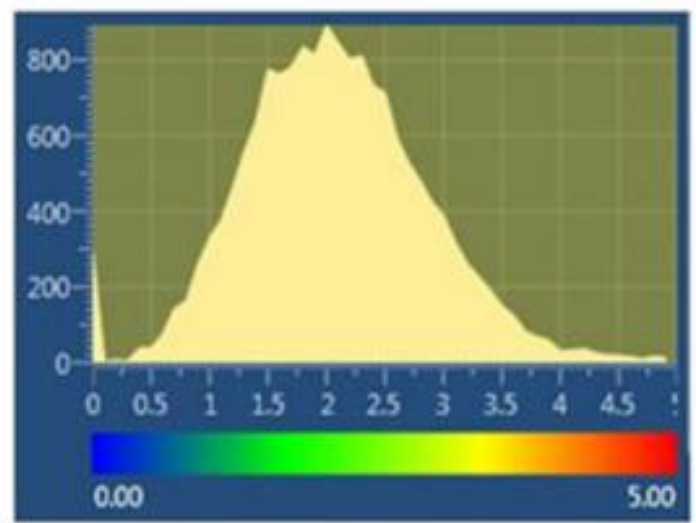

(d)

(f)

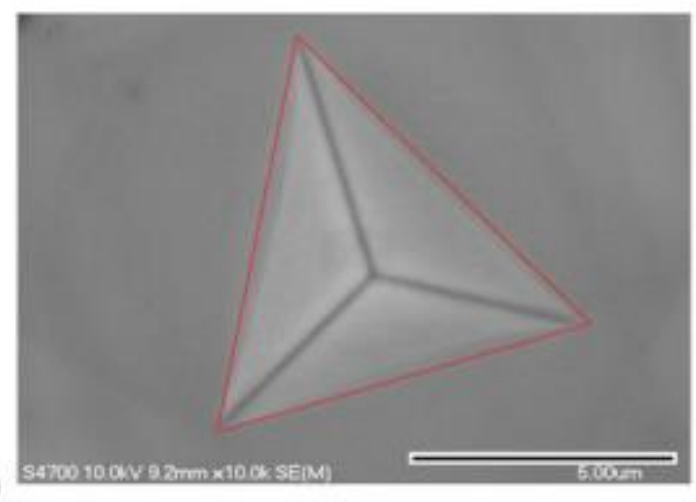

Figure 3.2. (a) Sample preparation methodology produces a large grain $(>1 \mathrm{~mm})$ sample with excellent quality map EBSD images, (b) KAM distribution plot for well-annealed surfaces, and (c) KAM images for mechanically polished surfaces that will include a contribution from the excess dislocations produced from the polish. (d) KAM plot for the 
mechanically polished surface and (e) well-annealed grain interior load:displacement curves showing the displacement discontinuity generally expected for dislocation nucleation process at surface with a very low preexisting dislocation density, (f) Secondary electron SEM image of a grain interior indentation The contact area is measured from the image and compared with the area function calculation from the reported indentation depth. The difference in the area measurement and area function result of $2.4 \%$ indicates the approximation of $h=h_{c}$ involves only a small error.

fraught with experimental challenges, such as, but not limited to, intermittent contact [32, $33,34]$, plasticity effects $[33,34,35]$, time-dependent flow [34, 35, 36], and extrapolation of the frame stiffness [34]. Based on the uncertainty associated with these potential experimental problems and the result of Fig. 3.2€, we have adopted this simplified definition of the contact depth. Accordingly, the elastic deflection of the surface is ignored, and $\mathrm{H}$ can, therefore, be calculated as a continuous function of depth based solely on the acquired $\mathrm{P}-\mathrm{h}$ data $[31,37]$. While ignoring the elastic deflection clearly reduces the accuracy of the estimated hardness, it is not immediately clear the error imposed by the simplified definition is any worse than the potential error imposed by the experimental challenges mentioned above. Moreover, any intermittent contact, which is particularly difficult to eliminate at small depths without paying a very significant penalty in the signalto-noise ratio, would completely eliminate the opportunity to measure the intrinsic ISE.

Experiments were performed with two indenter orientations relative to the grain boundary and within the adjacent grain interiors. Figure 3.1(a) is a backscatter scanning electron microscopy (SEM) image of a remote grain boundary indentation with the indenter tip positioned $\sim 2 \mu \mathrm{m}$ from the grain boundary and the flat face of the indenter parallel to the boundary. In this orientation, plastic deformation is induced in the indented grain first and dislocations can be treated as being pushed against the grain boundary as the plastic zone expands with increasing h. Alternatively, Fig. 3.1(b) shows a local grain boundary indentation, wherein the indenter tip is positioned less than $0.5 \mu \mathrm{m}$ from the grain boundary 
with the "wedge" of the indenter being perpendicular to the grain boundary plane. In this orientation, there is a near simultaneous deformation of both

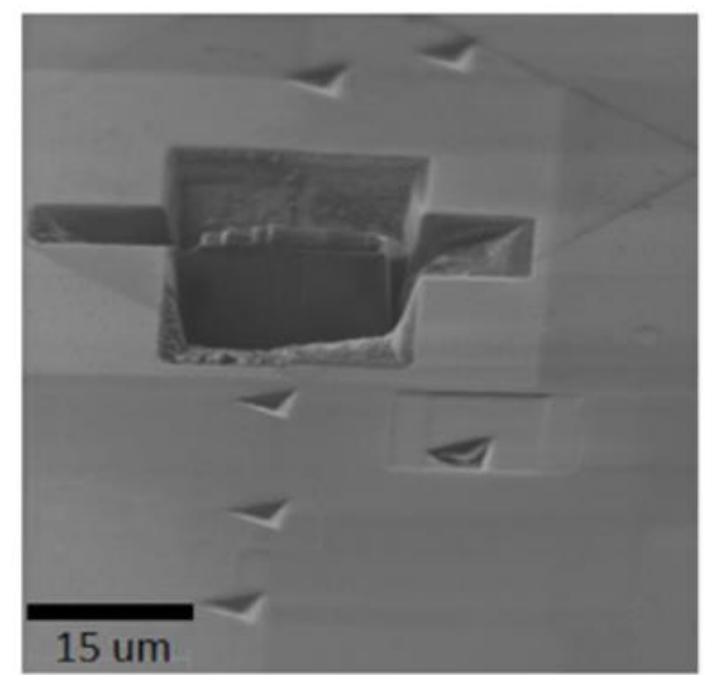

(a)

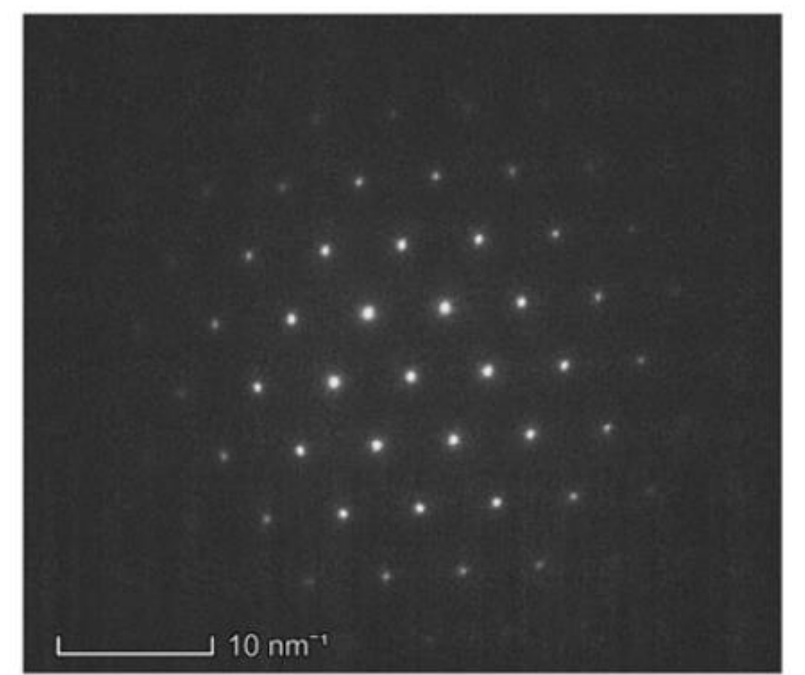

(b)

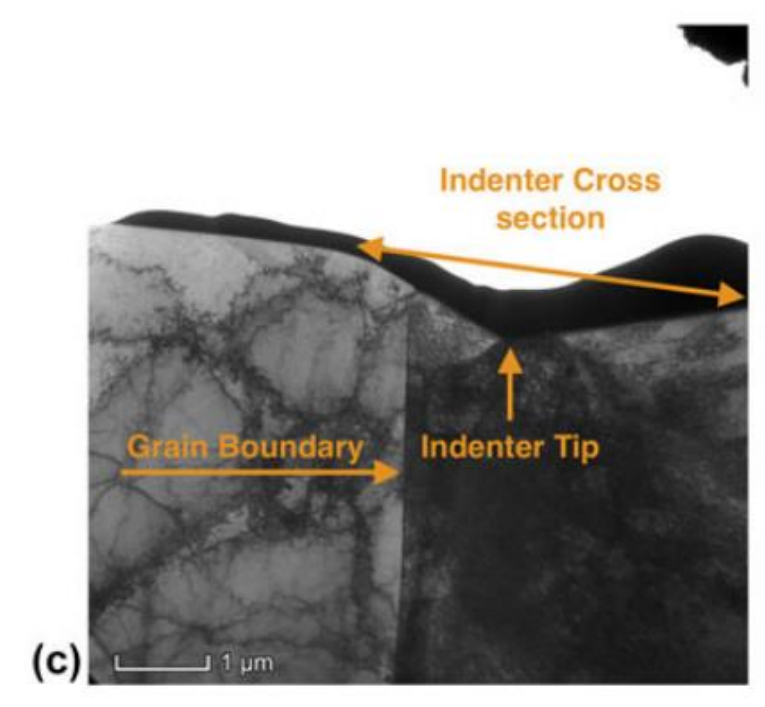

Figure 3.3. (a) FIB cut on the grain boundary. (b) Fe electron diffraction shows no evidence of carbides or other second phase precipitates. (c) Local indentation grain boundary crosssection indicates grain boundary plane approximately perpendicular to the surface. 
grains adjacent to the boundary due to direct contact of the indenter with the grain surfaces. A unique difference in the extrinsic ISE was observed for these two different indenter-grain boundary orientations, as shall be discussed in the Analysis section. The intrinsic ISE was determined using 20 indentations from the grain interiors adjacent to the studied boundaries.

Preexisting deformation gradients relative to the free surface of the $\mathrm{H}_{2}$ : $\mathrm{Ar} \mathrm{Fe}$ were intentionally created to examine the validity of using changes of the measured ISE relative to the intrinsic ISE of a well-annealed $\mathrm{H}_{2}$ :Ar heat-treated grain. A "one-dimensional" deformation gradient with a dislocation density gradient perpendicular to the surface was produced by mechanically polishing the surface using a coarse grit of P800, P-1200, and P-2400 and fine polish using 1, 0.3, and $0.05 \mu \mathrm{m}$ alumina [13]. Due to cell wall formation and other plastic inhomogeneities, we realize that the resulting dislocation density will not uniformly decrease with increasing depth below the surface. Rather, here we assume changes in the average dislocation density within the plastic zone with increasing $\mathrm{h}$, which effectively serves as a smoothing function, defines the gradient. To complement our examination of changes in the ISE brought about by a one-dimensional deformation gradient, we created a two-dimensional gradient by placing a closely spaced linear array of 250 small indentations (residual depth of 100 and $700 \mathrm{~nm}$ spacing) within the grain interior of a well-annealed specimen. This geometry is expected to produce a cylinder of high dislocation density by virtue of the overlapping the plastic zones of the closely spaced, shallow indentations. In this case, the dislocation density gradient will be both perpendicular to the surface and perpendicular to the linear array of indentations. To examine the effect of this multidimensional deformation gradient, indentation experiments were performed local to the array, as shown in Fig. 3.4, where the arrows identify the linear array of indentations. 


\subsection{RESULT AND DISCUSSION}

\subsubsection{Grain interior indentation results}

It is observed from the load-depth $(\mathrm{P}-\mathrm{h})$ curves in Fig. 3.5(a) and averaged hardness versus depth $(\mathrm{H}-\mathrm{h})$ profile plots in Fig. 3.5(b) that the $\mathrm{H}$ for the "as-polished" $<120>$ grain surface and indentation row deformation is significantly greater than that for the fully annealed grain interior, especially at small indentation depths. It may also be seen that the $\mathrm{H}$ at the grain boundary for the well-annealed sample is greater than that for the corresponding adjacent grain interior of the well-annealed sample. The increase in $\mathrm{H}$ for the as-polished grain interior and indentation row is expected on the basis of FD strengthening (work hardening) of the surface due to mechanical polishing [38] and the overlapping plastic strain fields of the closely spaced, shallow indentations. The increase in $\mathrm{H}$ at the grain boundary is expected due to grain boundary strengthening mechanisms interacting with the indentation plastic deformation. This paper intends to go beyond these simple concepts to examine how the ISE at these defect structures can provide additional information concerning the deformation mechanism and defect distribution. The degree of the ISE can be determined by noting the decrease in $\mathrm{H}$ with depth or the increase in $\mathrm{H}^{2}$ with 1/h [Fig. 3.5(b) and 3.5(c)]. As seen in Fig. 3.5(b) and 3.5(c), the ISE is most obvious for the unannealed, mechanically polished specimen. According to the Nix-Gao model, $\mathrm{H}^{2}$ versus $1 / \mathrm{h}$ should be linear, with the slope corresponding to the decrease in GND density with depth. Only the well-annealed sample approaches linear behavior. Previous work [13] has suggested that the experimental observations of the hardening effect of the polishing FD density averaged over the indentation volume, $\overline{\rho_{F D}}$, can be accounted for by rewriting Eq. (3.1) as follows:

$$
H^{2}=H_{0}^{2}\left(1+\frac{\overline{\rho_{F D}}}{\rho_{S S D}}+\frac{h^{*}}{f^{3} h}\right)
$$

Equation (3.2a) is an extension of the concept introduced by Poole et al. [11] that the preexisting dislocation density in a sample can be accounted for by a linear addition 
with the dislocations density produced by indentation. However, it is here considered that $\overline{\rho_{F D}}$ for the surface deformation due to polishing or from the row of shallow indentations is a function of h. Solving Eq. (3.2a) explicitly for $\overline{\rho_{F D}}$ as a function of indentation depth gives

$$
\frac{H^{2}-H_{0}^{2}\left(1+\frac{h^{*}}{f^{3} h}\right)}{(3 \sqrt{3} \alpha G b)^{2}}=\overline{\rho_{F D}}
$$

where $\mathrm{H}$ has been measured as function of $\mathrm{h}$. The functional form for $\overline{\rho_{F D}}$ can be determined by comparing Eq. [3.2(b)] with cold worked and annealed $\mathrm{H}-\mathrm{h}$ data in Fig. 3.5(c). Examining the annealed grain interior data first, such that $\left[\overline{\rho_{F D}}=\rho_{S S D}\right] \sim 0$ in Eq. 3.2(a), a linear best fit for the $\mathrm{H}^{2}$ versus $1 / \mathrm{h}$ of the $\langle 120\rangle$ grain surface for Eq. 3.2(a) determines that $\mathrm{H}_{\mathrm{o}}=1.05 \mathrm{GPa}$ and $\mathrm{h} * / \mathrm{f}^{3}=\sim 0.15 \mu \mathrm{m}$. Although this $\mathrm{h} * \mathrm{f}^{3}$ length scale is relatively small compared with most reported values for pure metals, it does match up well with the length scale $\left(\mathrm{h}^{*} \sim 0.1 \mu \mathrm{m}\right)$ reported for electropolished single-crystal $\mathrm{Cu}$ studies carried out by Liu et al. [10] and Ni single crystal (annealed or electropolished) studies carried out by Wang $\left(\mathrm{h}^{*} \sim 0.2-0.25 \mu \mathrm{m}\right)$ [7]. In both of these previous studies, it is apparent that both the $\mathrm{H}$ and the $\mathrm{h}^{*}$ length scale are highly dependent on the sample preparation procedure, especially when compared with poorly prepared surfaces [8]. 


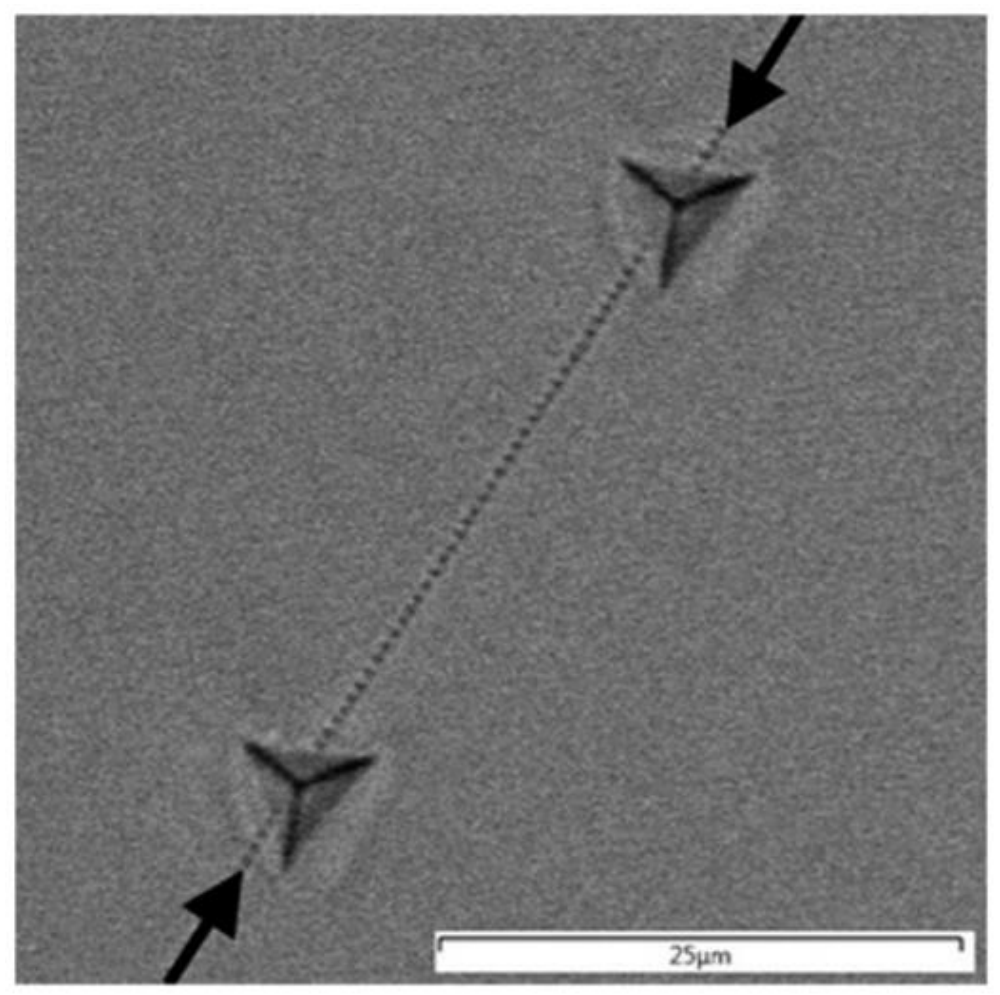

Figure 3.4. Backscatter SEM image for a linear array of closely spaced, shallow indentations with deeper indentations probing the properties using the local geometry similar to Figure 3.1(b).

That is, the nanoindentation of mechanically polished surfaces may measure an extrinsic size effect due to the gradient in FD density rather than the expected intrinsic ISE. This type of error in sample preparation can result in a larger measured internal length scale $\left(\mathrm{h}^{*}\right)$ due to the decrease in $\overline{\rho_{F D}}$ with indentation depth [13] as the slope of the $\mathrm{H}^{2}$ versus $1 / \mathrm{h}$ plot becomes

$$
\text { Slope }=H_{0}{ }^{2}\left(\frac{h^{*}}{f^{3}}+\frac{1}{\rho_{S S D}} \frac{d \overline{\rho_{F D}}}{d\left(\frac{1}{h}\right)}\right)
$$

rather than the expected intrinsic internal length, $\mathrm{h} * / \mathrm{f} 3$. 

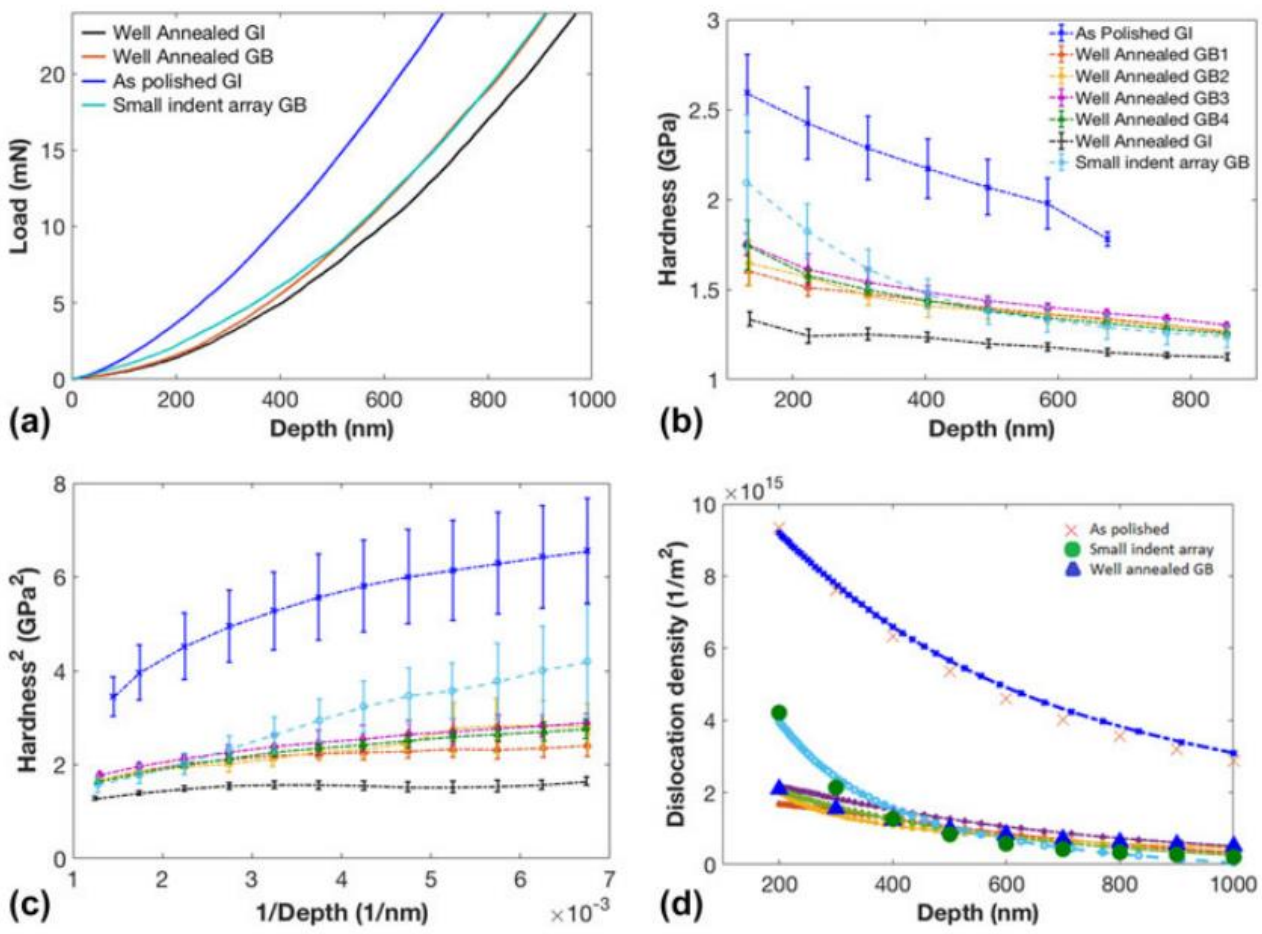

Figure 3.5. (a) Representative P-h curves for mechanically polished (deformed) grain surface, well-annealed grain surface, well-annealed grain boundary surface intersection, and line source indentation. (b) Plot between hardness and depth (averaged for $20 \mathrm{H}$ versus $\mathrm{h}$ curves at each condition) for all the processing conditions, indicating presence of ISE as a function of processing condition. The hardness of the grain boundary is also shown on the plot for comparison. (c) Nix-Gao based $\mathrm{H}^{2}$ versus $1 / \mathrm{h}$ plots for all the processing conditions. (d) Calculated FD density [Eq. (3.2)] for as polished surface, 4 grain boundaries as well as line source indentations. 


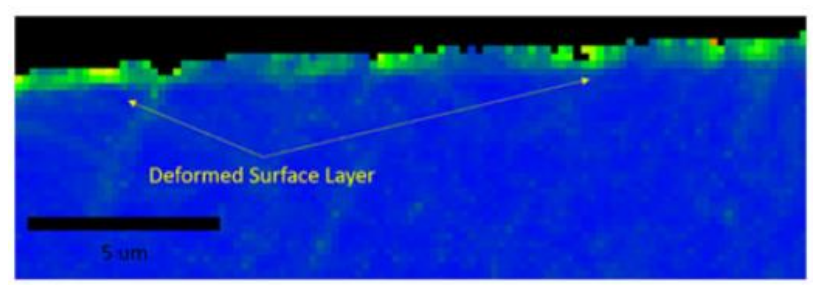

(a)

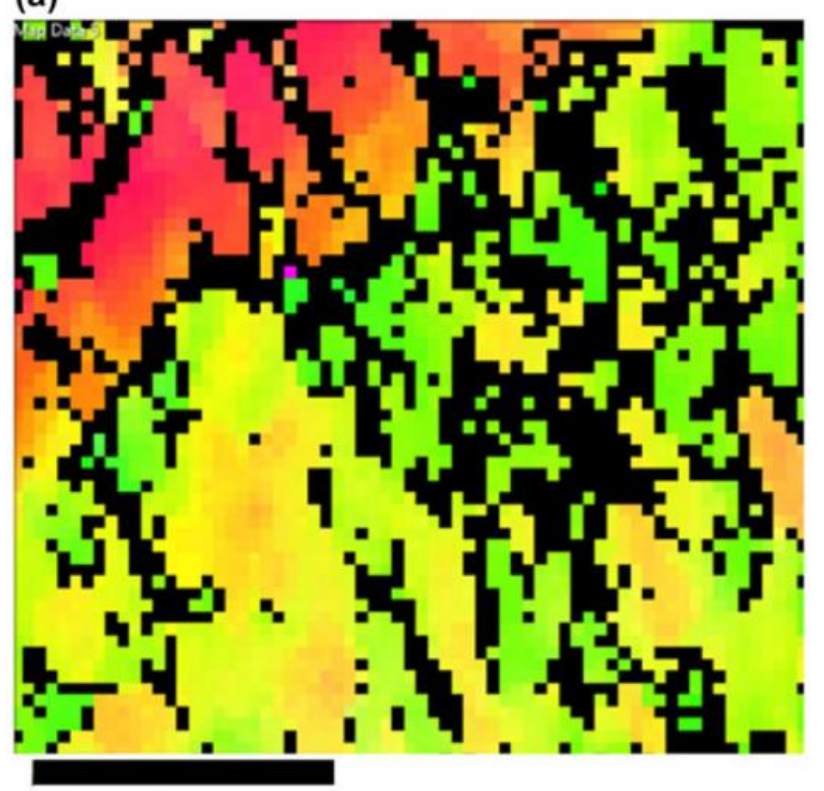

(b) 5 um

Figure 3.6. (a) KAM map of surface deformation cross section. (b) Plan view KAM map of as-polished surface.

With the result of $\mathrm{H}_{\mathrm{o}}=1.05 \mathrm{GPa}$ and $\mathrm{h} * / \mathrm{f}^{3}=\sim 0.15 \mu \mathrm{m}$ for this grain, the extrinsic ISE both in the mechanically polished surface and shallow indentation row (Fig. 3.4) samples can now be considered by further application of Eq. 3.2(a) and 3.2(b). The supposed depth dependence of $\overline{\rho_{F D}}$ can be determined by comparing Eq. 3.2(a) and 3.2(b) with the $\mathrm{H}^{2}$ versus $1 / \mathrm{h}$ data in Fig. 3.5(c), with the details of this analysis described elsewhere [13]. The result for $\overline{\rho_{F D}}$ is shown in Fig. 3.5(d). Although no independent measure of dislocation density is presented here, the maximum dislocation density 
predicted for the row of shallow indents in Fig. 3.5(d) is on the same order as that reported for Berkovich indentation of other body centered cubic metals [39, 40, 41].

The calculated $\overline{\rho_{F D}}$ variation with $\mathrm{h}$ in the as-polished condition is qualitatively consistent with the EBSD KAM map of the ion mill cross section of a deformed surface layer, as shown in Fig. 3.6(a). The deformation layer thickness indicated by the KAM map of the cross section is on the order of $1 \mu \mathrm{m}$ and the effect of such a length scale is considered in a more quantitative fashion in Eq. (3.6). As expected, the KAM map of the mechanically polished surface plane shows extensive distortion [Fig. 3.6(b)]. Furthermore, the relationship between Fig. 3.6(a) and the interpretation in Fig. 3.5(d) was supported by examining the change in the extrinsic ISE as a function of heat treatment [13], where the observed reduction in $\mathrm{H}$ and reduction in KAM map distortion due to FD recovery during annealing were successfully analyzed by the Nes dislocation recovery model [42].

\subsubsection{Grain boundary indentation results}

The modified Nix-Gao theory presented in Eq. 3.2(a) and 3.2(b) was used to rationalize the mechanical polished grain interior indentation results in Fig. 3.5(d) by invoking a depth dependence in the FD density. The general approach introduced in Eq. (3.2) is now extended to the analysis of the extrinsic ISE of an isolated grain boundary. As reviewed above, nanoindentation studies of grain boundary mechanical behavior are certainly not new. Much attention has been devoted to the examination of how indentation

just off the grain boundary in one grain must induce plasticity in the adjacent grain via slip transmission across the boundary. We have termed this remote indentation [Fig. 3.1(a)]. Such experiments can show $\mathrm{P}-\mathrm{h}$ curve discontinuities proposed to occur at the point of slip transmission [18], corresponding to a local maximum in the $\mathrm{H}-\mathrm{h}$ curve. 

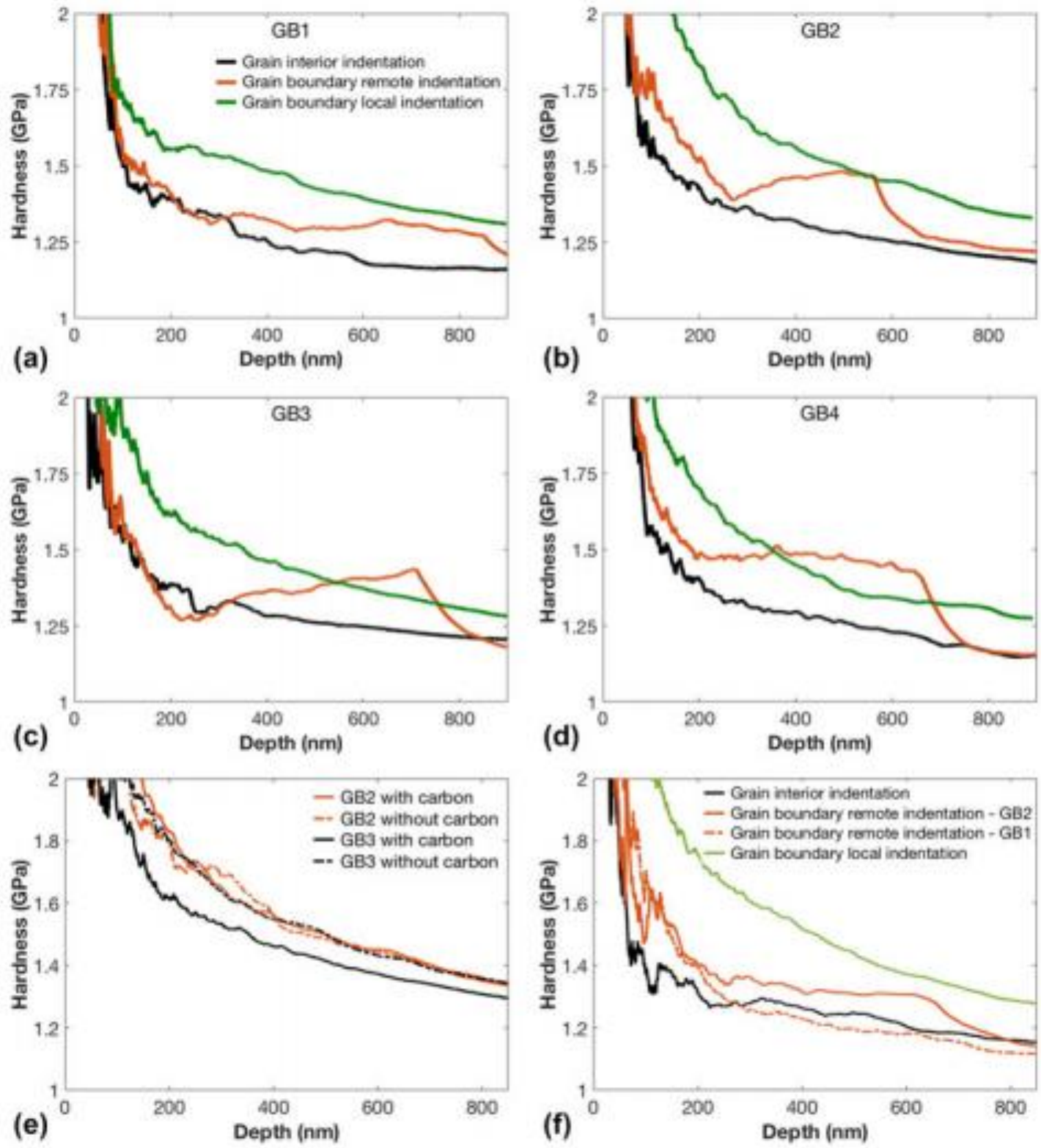

Figure 3.7. Representative hardness versus depth curves for 4 different grain boundaries in a well-annealed test specimen. (a) - (d) Show indentation results from the grain interior, the remote grain boundary indentation and the local grain boundary indentation for the carbon doped chemistry. (e) Comparison of local indentations for the same grain boundaries with and without carbon doping. (f) Comparison of local and remote grain boundary indentations with grain interior indentations with no carbon doping. 
These observations have been particularly prevalent in indentation of BCC metals and in particular Fe-C alloys, even with carbon content below the cementite solubility limit [43]. However, the observation of local maximum in $\mathrm{H}$ due to remote indentation is not specific to BCC materials [6], although reports of discontinuity in $\mathrm{P}-\mathrm{h}$ curves associated with such local maxima do appear to be specific to BCC materials. The focus of this paper is on what we have termed "local" indentation [Fig. 3.1(b)], where both adjacent grains undergo surface indentation at very small $\mathrm{h}$.

Figure 3.7 contrasts the $\mathrm{H}-\mathrm{h}$ behavior for local and remote indentation in carbondoped and $\mathrm{H}_{2}$ :Ar heat-treated Fe. Figures 3.7(a) - 3.7(d) contrasts local and remote indentation $\mathrm{H}-\mathrm{h}$ behavior at 4 different grain boundaries in carbon-doped Fe. The difference between local and remote indentation is accentuated by the local maximum in $\mathrm{H}$ observed for the remote indentation. The extensive reverse ISE after $\mathrm{h}=50 \mathrm{~nm}$ is only observed for the remote indentation geometry. Similar statements may also be made concerning the significant discontinuity in the $\mathrm{H}-\mathrm{h}$ slope observed for the remote indentation $\mathrm{H}$ compared with the local indentation. It is also noted from further comparison of remote and local indentation $\mathrm{H}-\mathrm{h}$ in Fig. 3.7(a) - 3.7(d) that the $\mathrm{H}$ of the remote indentation curve falls below that of the local indentation $\mathrm{H}$ after the discontinuity and approaches the $\mathrm{H}$ of the grain interior. In remote indentation, the stress is "relieved" after yielding occurs at the grain boundary [18]. While for local indentation the high $\mathrm{H}$ is gradually reduced with deformation occurring simultaneously on both sides of the grain boundary. Collectively, these observations suggest two different hardening mechanisms are being probed by the two different indenter geometries (local versus remote). Although not commonly described in the literature, this conclusion concerning the role of indentation geometry at the grain boundary should not be surprising, as a distinction between grain boundary effects on yield point and grain boundary hardening post yield is well known [44] for general, bulk deformation. 
The remote indentation causes a progressive increase in equivalent stress at the grain boundary as indentation depth increases and the stress at the grain boundary eventually reaches an equivalent stress that causes plastic strain to initiate across the boundary. As such, the extrinsic ISE observed due to grain boundary remote indentation is measuring the grain boundary resistance to dislocations crossing the boundary, being emitted from the boundary and/or generated from a dislocation source across the boundary. This is effectively examining the plastic yield point at the grain boundary as envisioned by Hall [45] and Petch [46]. On the other hand, local indentation causes plastic deformation on both sides of the grain boundary early in the indentation process. As the local indentation will induce near simultaneous dislocation motion on non-coincident slip systems in the crystals on either side of the grain boundary, the result is expected [3] to be incompatible shears within the indentation plastic volume. The strengthening observed for the local indentation of the grain boundary would, thus, be seen as probing the work hardening mechanism (post yield) of the grain boundary as originally envisioned by Ashby [3]. The Ashby GND mechanism for accommodation of shear incompatibility across the grain boundary is certainly one of the more accepted mechanisms proposed for work hardening at the grain boundary. Hansen [47] has utilized Ashby's work on the local deformation at grain boundaries due to plastic strain incompatibility to analyze the experimental results and develop a grain boundary work hardening model with the same form as the Hall-Petch equation. This mechanism is still utilized in some computer simulations [48] of polycrystalline plasticity as a solution to the Taylor-Sachs dichotomy. Also, these ideas continue to be developed in the more recent literature through self-consistent deformation models of polycrystalline materials [49] and within the development of nonlocal constitutive models [50].

Although not the focus of this paper, Fig. 3.7(e) compares local indentation $\mathrm{H}-\mathrm{h}$ curves for the same grain boundaries in annealed $\mathrm{H}_{2}$ :Ar specimens versus the carbon-doped specimens, indicating the $\mathrm{H}-\mathrm{h}$ curves are statistically indistinguishable between the two. Figure 3.7(f) gives examples of remote and local indentation of $\mathrm{H}_{2}$ :Ar heat-treated Fe. We 
note that some indentation curves do show the "hardening-softening" behavior reported for remote indentation of $\mathrm{Al}$ [6] but without the sharp discontinuity seen in Fig. 3.7(a) 3.7(d). Comparisons between Fig. 3.7(a) - 3.7(d) and Fig. 3.7(e) and 3.7(f) are consistent with the concept that the observations of local indentation $\mathrm{H}-\mathrm{h}$ are due to plastic strain geometry rather than grain boundary chemistry, while remote indentation results can be effected by grain boundary chemistry. Further support for the Ashby [3] interpretation is also presented in Appendix 3.B. However, a full analysis of this comparison of chemistry effects is not attempted in this paper and the focus is on the grain boundary hardness when plastic deformation is induced (approximately) simultaneously in both grains when the indenter tip is very close ("local") to the grain boundary $(0-0.5 \mu \mathrm{m})$ and the wedge of the indenter overlaps into the adjacent grain at small indentation depths.

The indentation behavior of an isolated grain boundary compared with grain interior behavior is shown in Fig. 3.5(a) - 3.5(c). The increase in $\mathrm{H}$ and bilinear $\mathrm{H}^{2}$ versus $1 / \mathrm{h}$ behavior specifically due to interaction with the grain boundary is isolated in Fig. 3.5(c). The increase in $\mathrm{H}$ with increase in $\frac{1}{\sqrt{h}}$ is suggestive of the Hall-Petch relationship. Historically, the three rationalizations for the classic Hall-Petch equation are pileup models [45, 46]: (i) work hardening-based models [3, 47] and (ii) grain boundary source models [51]. Work hardening models correlate the increase in dislocation density due to the grain boundary to the flow stress. These work hardening models are particularly applicable to grain boundary effects on the flow curve at significant plastic strain and not generally considered applicable to grain boundary effects on yield strength. It is proposed that these work hardening models [3,47] will be most relevant to the local indentation geometry [Fig. 3.1(b)], given the initiation of plastic deformation on both sides of the grain boundary at low indentation depths. Following the approach in Eq. 3.2(a) and 3.2(b), the effective depth dependence of a dislocation density that would correspond to the hardening response for indentation at a well-annealed grain boundary (GB2) may be calculated, as shown in Fig. 3.5(d). The calculated decrease in dislocation density with increasing $h$ does reflect the extrinsic ISE at the grain boundary. We consider if such a size effect could be rationalized 
on the basis of the plastic strain gradient magnitude required at the boundary to accommodate local deformation incompatibility across the grain boundary [3]. The scalar, "averaging" approach outlined by Nix and Gao [1] for the determination of the GND density for Berkovich indentation, is reduced to a single parameter (scaler measure) plastic displacement gradient (plastic strain), $\nabla u=\tan (\theta)=\frac{h}{a}$, due to the slope of the indenter: surface contact. Interestingly, the number of GNDs is taken as a constant, h/b, with respect to the position under the indenter tip, although the authors point out that the strain gradient may not be uniform. This non-uniformity would certainly be consistent with SaintVenant's principle. For example, the Fourier series solutions to the biharmonic equations for the two-dimensional displacement compatibility conditions (plasticity or elasticity) have an exponential decay. It should also be noted that a grain boundary bisecting the indentation plastic volume will disrupt any semblance of spherical (axial) symmetry in the deformation field, even though the plastic volume itself may still be approximated as spherical. Extending the Nix-Gao [1] approach to a non-uniform strain gradient field with loss of axial symmetry, it is possible to consider the plastic displacement gradient to have an exponential form associated with the presence of the grain boundary as $\nabla u_{(g b)}=\gamma *$ $\exp \left(-\frac{y}{\gamma}\right)$, where $\gamma$ is the maximum shear strain due to the "slope" of the plastic displacement caused by grain "overlap" [3] related to plastic incompatibility, y is the direction perpendicular to the grain boundary, and $\gamma$ is the decay length for this strain. The $\nabla u_{(g b)}$ can be substituted for h/a in the original derivation [3] for the GND density, with the average dislocation loop length taken as $(\pi f h / 2)$, giving Eq. 3.3(a):

$$
\begin{aligned}
\overline{\rho_{G N D(g b)}} & =\frac{1}{\frac{2}{3} \pi[f h]^{3}} \int_{0}^{f h} \frac{\gamma}{b} e^{-y /(\lambda)} \frac{\pi f h}{2} d y \\
& =\frac{3 \gamma \lambda}{4 b(f h)^{2}}\left(1-e^{-f h / \lambda}\right)
\end{aligned}
$$

Alternatively, Eq. 3.3(a) can be rewritten as follows: 


$$
\begin{aligned}
\overline{\rho_{G N D(g b)}} & =\frac{1}{\frac{2}{3} \pi[f h]^{3}} \int_{0}^{f h} \frac{\gamma}{b f h} e^{-y /(\lambda)} \frac{\pi(f h)^{2}}{2} d y \\
& =\frac{3 \gamma \lambda}{4 b(f h)^{2}}\left(1-e^{-f h / \lambda}\right)
\end{aligned}
$$

where $(\gamma / b f h)$ is the $\rho_{G N D_{(g b)}}$ at the grain boundary (Ashby [3]) and $\pi(f h)^{2} / 2$ is an approximation for the small circle area (disc) that the dislocation "lines" intersect. Equations 3.3(a) and 3.3(b) utilize the same approach as the original Nix-Gao [1] treatment, as modified by Durst et al. [4], that the plastic deformation gradient imposed by the geometry of the indenter results in GNDs spread over some fraction of the indentation plastic volume. The Nix-Gao-Durst approach considers a volume of integration with radius $[(f h / \tan \theta)]$. Analogous to this idea is that the excess dislocations produced by the deformation at the grain boundary is spread over a fraction of the indentation plastic volume, $(f h)$, which is much less than the grain size as the grain size here is $>1 \mathrm{~mm}$ [Fig. 3.2(a)]. Further justification for using $(f h)$ as a proxy for grain size comes from the work of Meakin and Petch [52, 53], who considered that the dislocation slip length was some fraction of the grain diameter and thus the dislocation density is inversely proportional to the grain size. This was later confirmed by the works of Conrad et al. [54, 55]. The ISE can be interpreted in a similar manner as the grain size effect [52] in that the dislocation density due to indentation appears to have an inverse relationship with the contact radius. For indentation local to the grain boundary [Fig. 3.1(b)], the argument analogous to Meakin and Petch is that the slip length of the dislocation due to plastic strain at the grain boundary is some fraction of the indentation plastic zone diameter.

Equation 3.3(b) can be correlated with the idea of an extrinsic ISE by noting the $h$ dependence of the $\overline{\rho_{G N D_{(g b)}}}$. If the decay length, $\lambda$, is proportional to $h$, then the "standard" $1 / \mathrm{h}$ dependence for $\mathrm{H}^{2}$ is recovered. Utilizing the same approach as in Eq. 3.2(a) leads to 
Eq. 3.4(a), with $h_{g b}^{*}$ as the characteristic length scale for the extrinsic ISE due to the grain boundary [Eq. 3.4(b)].

$$
H^{2}=H_{o}^{2}\left(1+\frac{h^{*}}{h f^{3}}+\frac{h_{g b}{ }^{*}}{h f^{3}}\right)
$$

Where from Eq. (3.3)

$$
h_{g b}^{*}=\frac{1}{\rho_{S S D}} \cdot \frac{3 \gamma \lambda f}{4 b h}\left(1-e^{-\frac{f h}{\lambda}}\right)
$$

Such that, analogous to Eq. [3.1(a)-3.1(d)],

$$
H_{o}{ }^{2} \frac{h_{g b}{ }^{*}}{h f^{3}}=(3 \sqrt{3} \alpha G b)^{2} \overline{\rho_{G N D_{(g b)}}}
$$

It is noted that if $\lambda$ in Eq. 3.4(b) is proportional to $\mathrm{h}$, then a constant $h_{g b}{ }^{*}$ is recovered. The characteristic length scales, $\frac{h^{*}}{f^{3}}$ and $\frac{h^{*} g b}{f^{3}}$, can also be determined by matching Eq. (3.4) to the experimental $\mathrm{H}^{2}$ versus $1 / \mathrm{h}$ curves for the data corresponding to the grain surfaces, GB1, GB2, GB3, and GB4 [Table (3.1)], as shown in Fig. 3.5(c). The $\mathrm{H}_{\mathrm{o}}$ values ( $\mathrm{H}$ for "infinite" indentation depth) can be determined by extrapolating the $\mathrm{H}^{2}$ versus $1 / \mathrm{h}$ to $1 / \mathrm{h}=0$. A curve fit the grain boundary $\mathrm{H}^{2}$ versus $1 / \mathrm{h}$ curve over the indentation depths of 500-1000 nm gives $\mathrm{H}_{\mathrm{o}}=1.05 \mathrm{GPa}\left(\mathrm{R}^{2}=0.97\right)$. However, the substantial bilinearity of the grain boundary $\mathrm{H}^{2}$ versus $1 / \mathrm{h}$ curve produces a larger $\mathrm{H}_{\mathrm{o}}$ value when the grain boundary $\mathrm{H}^{2}$ at indentation depths of $200-500 \mathrm{~nm}$ are extrapolated to $1 / \mathrm{h}=0$. Thus, we limit the consideration of the grain boundary $\mathrm{H}^{2}$ curve to indentation depths greater than $500 \mathrm{~nm}$. 
With this approach, the $\frac{h^{*}}{f^{3}}$ is determined using the slope of the grain interior $\mathrm{H}^{2}$ versus $1 / \mathrm{h}$ plot and is reported in Table (3.1). Having determined $\mathrm{H}_{\mathrm{o}}$ and $\frac{h^{*}}{f^{3}}$ from the grain interior data of the harder adjacent grain, Eq. 3.4(a) can now be fit to the extrinsic component of the grain boundary $\mathrm{H}^{2}$ versus $1 / \mathrm{h}$ plot, where $\frac{h^{*} g b}{f^{3}}$ is the fitting parameter. If the Hall-Petch coefficient, $\mathrm{k}$, is specifically defined as a multiplier relating local dislocation density to the inverse square root of grain size $[3,47,55,56]$ and $f h$ as a proxy for grain size, then Eq. 3.4(c) gives the relation:

$$
\frac{k}{\sqrt{f}}=\frac{H_{O}}{3} \sqrt{\frac{h^{*} g b}{f^{3}}} \frac{1}{\sqrt{f}}
$$

where the factor $1 / 3$ in Eq. (3.5) relates the grain boundary $H$ as three times the uniaxial stress at the same strain (Tabor's relationship). The curve fit results for $f=1$ are shown in Table (3.1) and show values for $\mathrm{k}$ of the same order as that determined by bulk mechanical testing of low carbon and interstitial free steels $\left(\mathrm{k}=0.11 \mathrm{MPa} \mathrm{m}^{1 / 2}\right)$ [57]. Alternatively, f may be calculated from Eq. (3.5) using the graphically determined values of $\mathrm{H}_{\mathrm{o}}$ and $\frac{h^{*} g b}{f^{3}}$ in Table (3.1) together with the experimental result [42] of $\mathrm{k}=0.1 \mathrm{MPa} \mathrm{m}^{1 / 2}$, giving the range $\mathrm{f} \sim 2.0$ to 3.2 . This range in $\mathrm{f}$ does bracket the value of the contact radius to indentation depth ratio for a cone geometry with included angle of $70^{\circ}, \frac{a}{h}=\tan (\theta)^{-1}=$ 2.74, suggesting that the grain size proxy is the same order as the contact radius. As support for the utilization of Eq. (3.5), it should be noted that Rester et al. [14] have introduced the concept that GNDs caused by the indentation geometry will form cell walls or sub-grains due to dynamic recrystallization and it is these "grain boundaries" under the indenter contact area that result in the intrinsic ISE. Although Rester et al. [14] consider the grain boundary strengthening is due to dynamic recrystallization as a "substitution" for the GND 
strengthening, the general idea of grain boundary strengthening due to a preexisting grain boundary under the indentation contact area is a natural extension of their analysis.

It is of interest to extend the concepts leading to Eq. 3.3(b) to the extrinsic, oneand two-dimensional dislocation distributions described in Fig. 3.5, determined from the procedure described in Soman et al. [13]. In contrast to Eq. 3.3(b), 3.2(a), and 3.2(b) do

\begin{tabular}{|c|c|c|c|c|c|c|c|}
\hline \multirow{2}{*}{$\begin{array}{c}\text { Grain } \\
\text { boundary }\end{array}$} & \multicolumn{2}{|c|}{ Misorientation } & \multirow{2}{*}{$\begin{array}{c}\mathbf{k} \\
\left(\text { Mpa.m }^{1 / 2}\right)\end{array}$} & \multirow{2}{*}{$\frac{\boldsymbol{h}^{*} g b}{f^{3}}$} & \multirow{2}{*}{$\frac{\boldsymbol{h}^{*}}{\boldsymbol{f}^{3}}$} & \multirow[t]{2}{*}{$\mathbf{H}_{\mathbf{o}}$} & \multirow{2}{*}{$\begin{array}{l}\mathbf{R}^{2} \text { for } \\
\frac{h^{*} g b}{f^{3}}\end{array}$} \\
\hline & Angle & Axis & & & & & \\
\hline GB1 & 27.37 & {$\left[\begin{array}{lll}\overline{3} & 2 & \overline{1}\end{array}\right]$} & 0.16 & 217.98 & 196.20 & 1.05 & 0.9668 \\
\hline GB2 & 26.18 & {$\left[\begin{array}{lll}\overline{2} & 4 & \overline{1}\end{array}\right]$} & 0.16 & 215.46 & 149.24 & 1.05 & 0.9999 \\
\hline GB3 & 25.43 & {$[\overline{1} \overline{2} 4]$} & 0.18 & 251.04 & 144.93 & 1.06 & 0.9655 \\
\hline GB4 & 44.92 & {$\left[\begin{array}{lll}\overline{3} & 4 & 4\end{array}\right]$} & 0.14 & 155.94 & 207.30 & 1.05 & 0.9954 \\
\hline
\end{tabular}

Table 3.1. Analysis of intrinsic and extrinsic ISE related to specific grain boundaries.

not interrogate the dimensionality of the preexisting dislocation distributions, although the results from Eq. 3.2(a) and 3.2(b) should be self-consistent with such an approach if there is any inherent validity. One issue is that the approximation of indentation strain spherical symmetry is broken when considering the dimensionality of the preexisting defect distributions studied here. It is thus suggested that dislocation density averaging is most straightforward using the Cartesian coordinate system such that 


$$
\bar{\rho}=\frac{3 \rho_{o}}{2 \pi(f h)^{3}} \int_{0}^{f h} \int_{0}^{f h} \int_{0}^{f h} S \cdot \exp \left(-\frac{x}{\lambda_{x}}\right) \exp \left(-\frac{y}{\lambda_{y}}\right) \exp \left(-\frac{z}{\lambda_{z}}\right) d x d y d z
$$

where $\rho_{\mathrm{o}}$ is the maximum dislocation density in the vicinity of the surface origin ( $\mathrm{x}$ $=0, \mathrm{y}=0, \mathrm{z}=0)$. The value $\mathrm{S}=8 \pi / 3$ is determined, so $\rho_{\mathrm{o}}$ is returned if all the directiondependent decay lengths, $\lambda_{\mathrm{i}}$, go to infinity, which would be equivalent to uniform deformation. The values of the decay lengths $\lambda_{\mathrm{i}}$ thus set the dimensional dependence of the extrinsic ISE. Specifically, for the one-dimensional dislocation density gradient (surface layer deformation, Fig. 3.6), only the direction normal to the surface will have a finite decay length as in Eq. 3.7(a).

$$
\frac{3 \rho_{o}}{2 \pi(f h)^{3}} \int_{0}^{f h} \int_{0}^{f h} \int_{0}^{f h} S \cdot \exp \left(-\frac{x}{\lambda_{x}}\right) d x d y d z=\rho_{o} \frac{4 \lambda_{x}\left(1-e^{-\frac{f h}{\lambda}}\right)}{f h}
$$

For the row of closely spaced shallow indentations, the preexisting distribution from the overlapping indentations is taken to have cylindrical symmetry extending along the z-axis. Thus, the surface normal direction $(x)$ and the orthogonal direction $(y)$ will have decay lengths that are finite and equal $\lambda_{x}=\lambda_{y}$ [Eq. 3.7(b)].

$$
\frac{3 \rho_{o}}{2 \pi(f h)^{3}} \int_{0}^{f h} \int_{0}^{f h} \int_{0}^{f h} S \cdot \exp \left(-\frac{x}{\lambda_{x}}\right) \exp \left(-\frac{y}{\lambda_{y}}\right) d x d y d z=\rho_{o} \frac{16 \lambda^{2} \sinh \left(\frac{h}{2 \lambda}\right)^{2}}{(f h)^{2}} e^{-\frac{f h}{\lambda}}
$$


A comparison of Eq. 3.7(a) and 3.7(b) with the dislocation density results from Eq. 3.2(a) and 3.2(b) is shown in Fig. 3.5(d), with $\frac{\lambda}{f}=193 \mathrm{~nm}$ and $\rho_{o}=7 \times 10^{15} \mathrm{~m}^{-2}$ for the surface deformation and $\frac{\lambda}{f}=125 \mathrm{~nm}$ and $\rho_{o}=2.4 \times 10^{16} \mathrm{~m}^{-2}$ for the indentation row deformation. This comparison shows that the proposed approach is consistent with observed $1 / \mathrm{h}$ dependence for the one-dimensional (surface) deformation gradient versus the observed $1 / \mathrm{h}^{2}$ dependence for the two-dimensional (indentation row) deformation gradient. It should be noted that although GNDs may be present within these fixed distributions related to prior deformation, these are not specific to the indentation geometry in the present experiment. In any case, the excellent fit for Eq. (3.6) and (3.7) in Fig. 3.5(d) suggests the extrinsic ISE for these preexisting defects is apparently controlled by their inherent distribution and dimensionality prior to the indentation process.

When considering the grain boundary indentation using the approach leading to Eq. (3.6) and (3.7), the dislocation density distribution due to the presence of the grain is taken as decaying primarily perpendicular to the grain boundary plane (in one dimension) and is, thus, the same form (geometry) as for the surface deformation [Eq. 3.7(a)], but with a decay length proportional to the grain size, or in our case, h. This is consistent with the Ashby [3] theory of grain boundary GNDs. Again, following Ashby [3], the average GND density at the grain boundary is considered inversely proportional to $\mathrm{h}$ in Eq. 3.3(a) with dislocation loop length $(\pi f h)$. Using a proportionality constant, n, such that $\lambda=\left(\frac{f h}{n}\right)$, then Eq. (3.6) can be rewritten for the grain boundary case as follows:

$$
\left[\frac{\pi \gamma}{b f h}\right] \frac{3}{2 \pi(f h)^{3}} \int_{0}^{f h} \int_{0}^{f h} \int_{0}^{f h} S \cdot \exp \left(-\frac{y}{\lambda}\right) d x d y d z=\frac{4 \pi \gamma\left(1-e^{-n}\right)}{b f h n}
$$


As in the case for the one- and two-dimensional extrinsic deformation gradients, the integral equation solution [Eq. (3.8) with $\frac{4 \pi \gamma}{b f} *\left(\frac{\left(1-e^{-n}\right)}{n}\right)=4.5 \times 10^{8} \mathrm{~m}^{-1}$ ] well represents the dislocation density distributions determined from the extrinsic ISE at one of the grain boundaries (GB2) in Fig. 3.5(d). The implications of the results for Eq. (3.5) and (3.8) are considered further in Appendix 3.B. In the Analysis section, a continuum SGP approach with considerably greater mathematical structure, relative to Eq. (3.8), is considered.

\subsubsection{Analysis: continuum SGP formulation and the SGP boundary condition related to the Ashby model}

The analysis developed here is not meant to be predictive but rather to be interpretive in exposing the physics of the additive effects of intrinsic and extrinsic ISE. As such, an approximate analytical approach is pursued using a two-dimensional argument with prescribed displacements (strains) within the context of a continuum SGP theory. The purpose of this exercise is to connect Eq. 3.4(a) to more specific considerations of the spatial dependence of plastic strain. A simple physical description of the Ashby [3] proposal will be utilized to develop an appropriate boundary condition for the linear continuum model in the case of anisotropic plastic shape change for the adjacent grains, designated 1 and 2. The approach centers around the relation between the plastic strain, ep, along a specific loading direction $(\mathrm{x})$ and the shear strain, $\mathrm{c}$, that occurs on specific crystallographic planes. For a scaler argument with single slip, the normal plastic strain component is given in terms of the shear and the product of the slip system direction cosines (m) as follows:

$$
\varepsilon_{p}=\gamma \cos (\theta) \cos (\phi)=\gamma m
$$


where $\mathrm{m}$ is also known as the Schmid factor. Alternatively, $\mathrm{m}=\frac{d \varepsilon_{p(x)}}{d \gamma}$, as defined by Taylor [19]. For indentation local to the grain boundary, it is expected that the plastic displacement at the surface will be equivalent in both grains at the grain boundary. But if $\mathrm{m}$ for grain 1 is different from $\mathrm{m}$ for grain 2 , then $\gamma_{1}$ will be different from $\gamma_{2}$ or,

$$
\gamma_{1}-\gamma_{2}=\varepsilon_{p} \frac{m_{1}-m_{2}}{m_{1} m_{2}}
$$

Ashby uses the difference in plastic shear strain across the grain boundary to determine the additional plastic displacement at the grain boundary, $u_{g b}$, required for plastic compatibility. This plastic displacement scales with the grain radius, D, as

$$
u_{g b}=\varepsilon_{p} D \frac{m_{1}-m_{2}}{m_{1} m_{2}}
$$

This plastic strain, $u_{g b} / \mathrm{D}$, is in addition to the imposed plastic strain, ep, which according to Taylor [19] would correspond to the operation of additional slip systems to allow for strain compatibility across the grain boundary. Ashby further implies that the excess plastic strain will decay with position away from the grain boundary by noting the development of $\rho_{G N D}$ at the grain boundary as

$$
\rho_{G N D}=\frac{u_{g b}}{b} \cdot \frac{1}{D L}=\frac{\varepsilon_{g b}}{b L} \cdot \Delta m
$$

Where, 


$$
\Delta m=\frac{m_{1}-m_{2}}{m_{1} m_{2}}
$$

where the width $\mathrm{L}$ is the distance from the grain boundary that contains the bulk of the GNDs and may be considered as the decay length of the excess plastic strain. This length scale, L, may be considered equivalent to that described in Evers et al. [48] for the grain boundary deformation layer thickness required for compatibility due to anisotropic plastic deformation. Evers et al. [48] considered L to be proportional (but not equal) to the grain radius, while Ashby [3] took this width to be equivalent to the grain diameter. Although the analysis to be developed here is not accurate enough to give a conclusive argument for either the Ashby [3] or Evers [48] approach to the decay length for the excess plastic strain, it is to be implicitly included.

The generally accepted relationship between the density of GNDs and the plastic strain gradient, apparently first proposed by Ashby [3], is as follows:

$$
\rho_{G N D}=\frac{1}{b}\left(\frac{d}{d y} \varepsilon_{p}\right)
$$

Using Eq. (3.14) with Eq. (3.12) gives

$$
\frac{\varepsilon_{p}}{L} \Delta m=\left(\frac{d}{d y} \varepsilon_{p}\right)
$$

which is used as a boundary condition at the grain boundary for the development of the continuum plasticity analysis to follow. The rhs of Eq. (3.15) can be interpreted as the increase in plastic strain due to a non-zero $\Delta \mathrm{m}$, which decays over the length, $\mathrm{L}$, as defined 
by the spatial derivative on the lhs of Eq. (3.15). The occurrence of an excess in plastic strain beyond the imposed plastic strain, Eq. (3.11), together with a gradient in plastic strain does suggest a peak in a scaler measure of plastic strain magnitude at the grain boundary. For dislocation density proportional to the square of the strain and proportional to the strain gradient, a significant increase in dislocation density is then expected at the grain boundary and the experimental results of Vachhani et al. [20] are seen to be particularly relevant.

The formulation for the SGP continuum analysis that can be adapted to the linear analysis pursued here is described in a recent publication by Wei et al. [58].

$$
\sigma=\beta\left(\overline{\varepsilon_{p}}+l_{1}\left[\vec{\nabla} \overline{\varepsilon_{p}} \vec{\nabla} \overline{\varepsilon_{p}}\right]^{q}+l_{2} \nabla^{2} \overline{\varepsilon_{p}}\right)
$$

where $\beta$ is the hardening modulus. For the analysis here, the characteristic length $l_{2}=0$ and the exponent $=1 / 2$, as represented by Eq. [3.A(1)] and [3.A(3)] in Appendix 3.A. The equivalent stress, $\sigma$, is a function of the equivalent plastic strain measures, $\overline{\varepsilon_{p}}=\sqrt{\left(\frac{2}{3}\right) \varepsilon_{i j}^{p} \varepsilon_{i j}^{p}}$ and the magnitude of the gradient squared $\left(\vec{\nabla} \overline{\varepsilon_{p}} \vec{\nabla} \overline{\varepsilon_{p}}\right)$ as utilized in Eq. (3.16), where $\varepsilon_{i j}^{p}$ is the plastic strain tensor. The details of the solution to Eq. (3.16) are given in Appendix 3.C.

The stress contribution from the grain boundary to the averaged equivalent stress at the surface and the extrinsic ISE is found to have the form:

$$
\left[\left.\overline{\sigma_{(g b)}}\right|_{x=\pi / 2 \omega}\right]_{\text {extrinsic }}=\left(\frac{0.12 n H_{o} l_{1} \Delta m}{h}\right)+0.02 n \beta \Delta m
$$


Drawing from the concepts leading to Eq. [3.A(8)] the total stress from the addition of the intrinsic and extrinsic components of the deformation is then used to estimate $\mathrm{H}$ :

$$
H=\left[H_{o}\left(1+\frac{h^{*}}{2 f^{3} h}\right)\right]_{\text {intrinsic }}+\left[\left.\overline{\sigma_{(g b)}}\right|_{x=\pi / 2 \omega}\right]_{\text {extrinsic }}
$$

Although the approach leading to Eq. (3.18) is linear, two dimensional and limited to a single Fourier coefficient, it is possible to still have a reasonable comparison with the experimental results obtained from local grain boundary indentation, as presented in Fig. 3.5 and 3.7. This comparison between Eq. (3.18) and the local indentation experimental results for 4 different grain boundaries is shown in Fig. 3.8 with

\begin{tabular}{|c|c|c|}
\hline Grain Boundary & $\boldsymbol{n} \boldsymbol{\beta} \Delta \boldsymbol{m}(\mathbf{G P a})$ & $\boldsymbol{n} \boldsymbol{H}_{\boldsymbol{o}} \boldsymbol{l}_{\mathbf{1}} \Delta \boldsymbol{m}(\mathbf{G P a . n m})$ \\
\hline GB1 & 7.0 & 45.0 \\
\hline GB2 & 8.0 & 90.0 \\
\hline GB3 & 10.0 & 105.0 \\
\hline GB4 & 8.0 & 82.5 \\
\hline
\end{tabular}

Table 3.2. Fit parameters for Eq. (3.18) in figure (3.8).

the two independent fit parameters detailed in Table (3.2). These two fit terms are multiplicative products of parameters but still may be judged as the same order as that 
determined in Eq. (3.4) [Table (3.2)] with the limiting case for the values of $\Delta \mathrm{m}$ examined in Appendix 3.B.

The extrinsic component of Eq. (3.18) can be compared with $\mathrm{H}_{\mathrm{o}} \frac{h_{g b}^{*}}{f^{3}}$ in Eq. (3.4) and Eq. [3.A(9)] as follows:

$$
\text { Ho } \frac{h_{g b}^{*}}{2 f^{3}}=\left(0.12 H_{o} l_{1}+0.02 \beta h\right) n \Delta m
$$
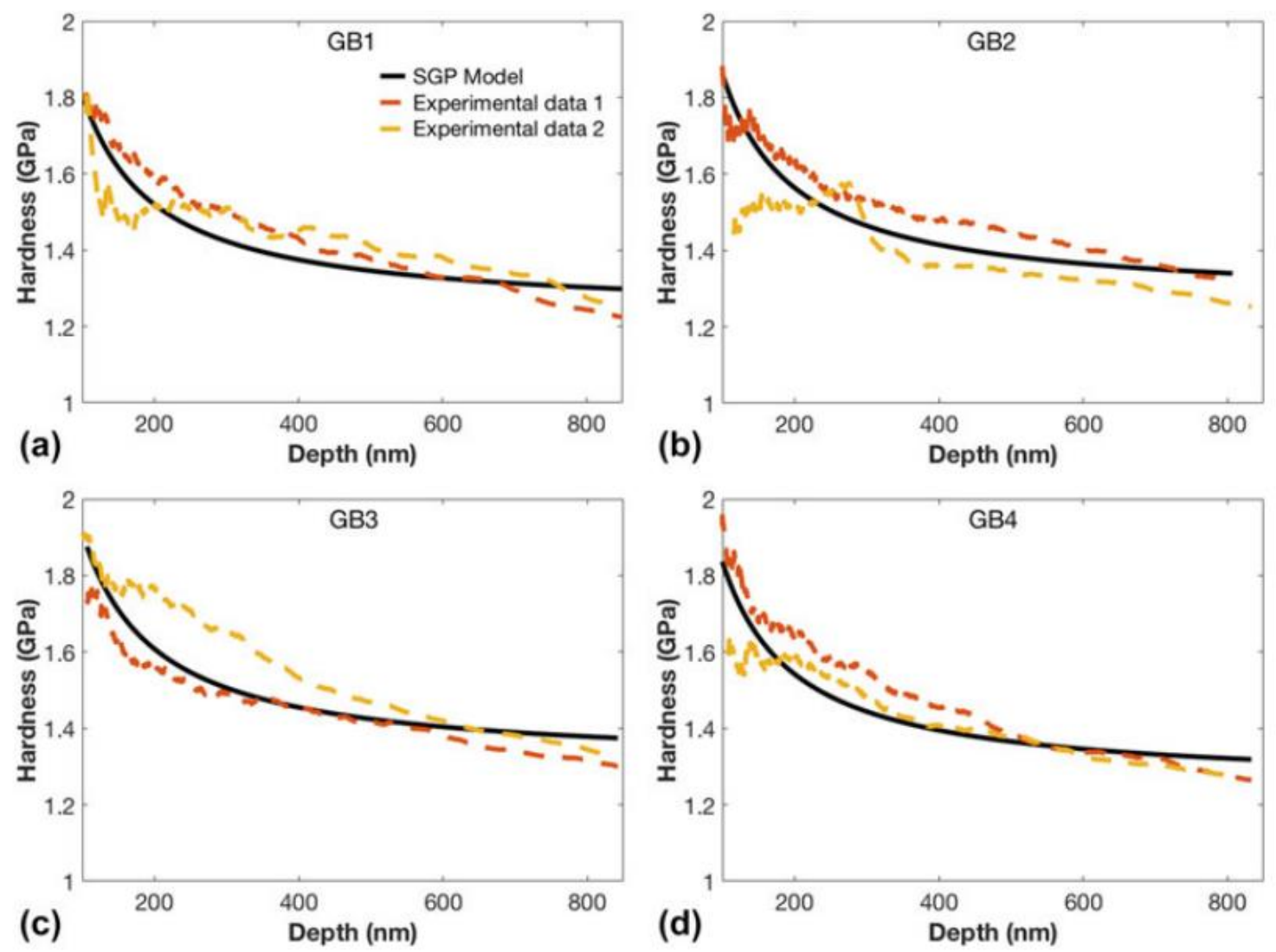

Figure 3.8. The comparison of the continuum SGP model Eq. (3.18) from the experimental results from local indentations on the 4 grain boundaries (a) - (d). Two experimental curves 
(dashed) from each boundary representing the variance of the behavior are presented. The solid curve is the fit from Eq. (3.18).

The nonequivalence of $\mathrm{h}_{\mathrm{gb}}$ and $\mathrm{l}$ in Eq. (3.19), as discussed in Appendix A, can be resolved by taking $H_{o}=\beta b \sqrt{\overline{\rho_{S S D}}}$ and noting from Appendix 3.A that $l_{1} \cong \frac{1}{\left(2 \sqrt{\overline{\rho_{S S D}}}\right)}$. Equation (3.19) provides the insight that the characteristic length, $\frac{h_{g b}^{*}}{f^{3}}$, can be described as a function of $h$. The possibility of strain dependence for the characteristic length in gradient theories has been considered previously $[58,59,60]$, but a specific discussion of $\frac{h_{g b}^{*}}{f^{3}}$ dependence on $\mathrm{h}$ has only been previously described in the work of Feng at al. [61], which considers the volume correction factor, $f$, as inversely proportional to $h$. This conclusion [61] is consistent with the finding in Eq. (3.19).

The parameter, $\left(H_{o} l_{1}\right) n \Delta m$, on the right-hand side of Eq. (3.19) has special significance as a description of (energy dissipation)/area, or mechanically induced interface energy, due to deformation at the grain boundary. This term describes the deformation energy cost for the formation of GNDs seen in Eq. (3.14) and (3.15). The physical basis of how the grain boundary GNDs contribute to the strengthening can be illustrated using Eq. [3.A(6)] and [3.A(7)] as follows:

$$
\sigma_{g b}^{G N D}=\beta b\left(\rho_{G N D} l_{1}\right)=\beta b\left(\frac{H_{o}}{\beta b h} l_{1}\right) n \Delta m
$$

Rewriting Eq. [3.20(a)] as 


$$
\sigma_{g b}^{G N D} h=\left(H_{o} l_{1}\right) n \Delta m
$$

where the rhs of Eq. [3.20(b)] is constant within the error of the curve fitting in Fig. 3.8, indicating the same for the lhs of Eq. 3.20(b). The values for $\left[\left(H_{o} l_{1}\right) n \Delta m\right]$ are listed in Table (3.2) and may be interpreted through the work of Aifantis and Willis [27, 28] who introduced (phenomenologically) a specific interface parameter that characterizes the grain boundary strength and is termed a, $\gamma$, or $\xi$ with units of $\mathrm{Pa} / \mathrm{m}^{2}$ or $\mathrm{N} / \mathrm{m}$. In a recent study [18], the value of $\xi$ was deduced by directly relating remote nanoindentation [Fig. 3.1(a)] data for grain boundaries in $\mathrm{Fe}-3 \mathrm{wt} \% \mathrm{Si}$. The values found for the $\mathrm{Fe}-3 \mathrm{wt} \% \mathrm{Si} \xi$ were 291-400 N/m, depending on the grain boundary type. Although the same order, as described in Table 3.2, a one to one comparison is difficult because the difference in alloy content and indentation geometry.

It should be noted that in interfacial gradient plasticity the interface parameter $(\xi)$ is introduced within a mechanical interface energy $\Phi$, which is a function of the plastic strain on the GB. Letting $\xi=\left(H_{o} l_{1}\right) n \Delta m$ would then imply that

$$
\Phi=\frac{\beta}{2} b\left(\left(\frac{H_{o}}{\beta}\right)^{2} \frac{l_{1}}{b}\right) n \Delta m
$$

where $\mathrm{H}_{\mathrm{o}} / \mathrm{b}$ is the indentation plastic strain for very large $\mathrm{h}$. Such a quadratic dependence of $\Phi$ on the square of the plastic strain was postulated for a grain boundary that begins deforming simultaneously as the adjacent grain [62], which is in fact the case in the present indentation experiments. Reports on these types of boundary energy dissipation terms have increased in development and application since the inception in a series of papers by Aifantis and Willis [62]. However, this appears to be the first instance, 
where a boundary dissipation term has been considered for the Ashby grain boundary deformation mechanism.

The comparison of Eq. (3.18) and (3.19) with Eq. (3.3), (3.4), and (3.8) allows us to close the loop on the arguments developed herein. The exponential dependence of plastic strain and dislocation density utilized in these equations is seen to be consistent with a solution based on the Marguerre function in Eq. [3.C(3a)]. Similarly, to determine the average equivalent stress under the indenter, the exponential decay of flow stress from the grain boundary position at $\mathrm{y}=0$ must be integrated, analogous to integrating the spatial variation (exponential decay) of dislocation density in Eq. (3.8). Finally, the solution to Eq. (3.16) in Appendix 3.C gives the plastic strain gradient at the grain boundary, proportional to the GND density, as

$$
\left|\nabla \overline{\varepsilon_{p}}\right|_{y=0} \propto\left|\frac{H_{o} \Delta m}{\beta L}\right|
$$

where it is reiterated that $\mathrm{L}$ can be considered proportional to h. Note that $H_{o}=\left.\beta \overline{\varepsilon_{p}}\right|_{\Delta m=0}$ and then Eq. (3.21) is consistent with Eq. (3.3), (3.4), (3.8), (3.12), and (3.15). The continuum SGP solution [Eq. (3.18)] does suggest that both the plastic strain and the strain gradient magnitude will increase at the grain boundary, indicating the grain boundary will be "harder" than the adjacent material for $\beta>0$. The local increase in plastic strain and plastic strain gradient magnitude, and thus dislocation density, at the grain boundary is also explicit in the integral kernel showing exponential decay of dislocation density in Eq. (3.3) and (3.8). However, the physical origin is only explicit in the continuum SGP solution and may be identified as the boundary condition of Eq. [3.C(7b)]. The "uniform" imposed plastic displacement at the surface-grain boundary intersection in Eq. [3.C(7b)] is reminiscent of the parallel loading for polycrystalline materials, as originally envisioned 
by Taylor [19], while the spatial variation in plastic strain is suggestive of the polycrystalline deformation process conceptualized by Sachs [63]. To support the result of spatial variation in stress and plastic strain (dislocation density), it is recalled that the experimental work of Vachhani [20] does show local grain boundary hardening occurs in deformed $\mathrm{Al}$ (as measured post yield), and this is consistent with the concept that the total dislocation density is increased there.

\subsection{CONCLUSION}

The extrinsic ISE for preexisting, one- and two-dimensional deformation gradients is rationalized through an extension of the Nix-Gao [1] integral averaging approach of dislocation densities to allow for explicit consideration of the dimensionality of the dislocation density distribution. This approach can be applied to the extrinsic ISE observed for local indentation of grain boundaries in Fe and carbon-doped Fe. Even though the extrinsic ISE due to the grain boundary can be modeled as a one-dimensional deformation gradient using this approach, the underlying mechanism causing the increase in grain boundary hardening could not be explicitly addressed. However, utilizing the Ashby [3] theory of grain boundary GND, a simple application of a continuum SGP model [58] is found to be qualitatively consistent with the integral kernel constructed for the dislocation distribution in the vicinity of a deformed grain boundary. Particularly, it was possible to interpret the interface parameter $\xi$ (which has been introduced within interfacial gradient plasticity) as being equal to the product of the hardness of the GND free grain, the $\Delta \mathrm{m}$, and the internal length. The simple continuum SGP analysis employed here also reveals the structure of the plasticity distribution at the grain boundary, suggesting that both the plastic strain and plastic strain gradient increase there. This continuum SGP result is consistent with previously published, independent experimental [20] and theoretical [48] analyses. 


\subsection{ACKNOWLEDGMENT}

This work was supported by Office of Basic Energy Sciences within the Department of Energy (DOE) Award Number: DESC0016314 and DE-SC0017715. 


\section{REFERENCE}

1. W.D. Nix and H. Gao: Indentation size effects in crystalline materials: A law for strain gradient plasticity. J. Mech. Phys. Solids 46, 411 (1998).

2. A.H.W. Ngan and H.P. Ng: Indentation-induced damage of thinfilms supported on substrates. In Advances in Fracture Research - 10th International Congress on Fracture (Pergamon, Oxford, 2001).

3. M.F. Ashby: The deformation of plastically non-homogeneous materials. Philos. Mag. 21,399 (1970).

4. F.K. Durst, B. Backes, and M. Goken: Indentation size effect in metallic materials: Correcting for the size of the plastic zone. Scr. Mater. 52, 1093 (2005).

5. X.D. Hou, A.J. Bushby, and N.M. Jennett: Study of the interaction between the indentation size effect and Hall-Petch effect with spherical indenters on annealed polycrystalline copper. J. Phys. D: Appl. Phys. 41 (2008).

6. G.Z. Voyiadjis and M. Yaghoobi: Review of nanoindentation size effect: Experiments and atomistic simulation. Crystals 7, 321 (2017).

7. Z. Wang: Influences of sample preparation on the indentation size effect and nanoindentation pop-in on nickel. Ph.D. dissertation, University of Tennessee, Knoxville, 2012. Available at: https:// trace.tennessee.edu/utk_graddiss/1371.

8. G.M. Pharr, E.G. Herbert, and Y. Gao: The indentation size effect: A critical examination of experimental observations and mechanistic interpretations. Annu. Rev. Mater. Res. 40, 271 (2010). 
9. K.W. McElhaney, J.J. Vlassak, and W.D. Nix: Determination of indenter tip geometry and indentation contact area for depthsensing indentation experiments. J. Mater. Res. 13, 1300 (1998).

10. Y. Liu and A.H.W. Ngan: Depth dependence of hardness in copper single crystals measured by nanoindentation. Scr. Mater. 44, 237 (2001).

11. W. Poole, M.F. Ashby, and N.A. Fleck: Micro-hardness of annealed and work hardened Cu polycrystals. Scr. Mater. 4, 559- 564 (1996).

12. B. Backes, Y. Huang, M. Goken, and K. Durst: The correlation between the internal material length scale and the microstructure in nanoindentation experiments and simulations using the conventional mechanism-based strain gradient plasticity theory. J. Mater. Res. 24, 1197 (2009).

13. P.P. Soman, E.G. Herbert, K.E. Aifantis, and S.A. Hackney: Effect of processing on Nix-Gao bilinear indentation results obtained for high purity iron. MRS Adv. 3, 477 (2018).

14. M. Rester, C. Motz, and R. Pippan: Microstructural investigation of the volume beneath nanoindentations in copper. Acta Mater. 55, 6427 (2007).

15. B-b. Jung, H-k. Lee, and H-c. Park: Effect of grain size on the indentation hardness for polycrystalline materials by the modified strain gradient theory. Int. J. Solids Struct. 50, 2719 (2013).

16. K.E. Aifantis and A.H.W. Ngan: Modeling dislocation-grain boundary interactions through gradient plasticity and nanoindentation. Mater. Sci. Eng., A 459, 251 (2007).

17. W.A. Soer, K.E. Aifantis, and J.T.M.D. Hosson: Incipient plasticity during nanoindentation at grain boundaries in bodycentered cubic metals. Acta Mater. 53, 4665 (2005). 
18. K.E. Aifantis, H. Deng, H. Shibata, H. Tsurekawa, S. Lejcek, and S.A. Hackney: Interpreting slip transmission through mechanically induced interface energies: A Fe-3\% Si case study. J. Mater. Sci. 54, 1831 (2019).

19. G. Taylor: Plastic strain in metals. J. Inst. Met. 62, 307 (1938).

20. S.J. Vachhani, R.D. Doherty, and S.R. Kalidindi: Studies of grain boundary regions in deformed polycrystalline aluminum using spherical nanoindentation. Int. J. Plast. 81, 87 (2016).

21. B. Yang and H. Vehoff: Dependence of nanohardness upon indentation size and grain size-A local examination of the interaction between dislocations and grain boundaries. Acta Mater. 55, 849 (2007).

22. X. Xiao, D. Terentyev, Q. Chen, L. Yua, L. Chen, A. Bakaev, and H. Duan: The depth dependent hardness of bicrystals with dislocation transmission through grain boundaries: A theoretical model. Int. J. Plast. 90, 212 (2017).

23. S. Pathak, J. Michler, and K. Wasmer: Studying grain boundary regions in polycrystalline materials using spherical nanoindentation and orientation imaging microscopy. J. Mater. Sci. 47, 815 (2012).

24. E.C. Aifantis: On the microstructural origin of certain inelastic models. J. Eng. Mater. Technol. 106, 326 (1984).

25. N.A. Fleck, G.M. Muller, M.F. Ashby, and J.W. Hutchinson: Strain gradient plasticity: Theory and experiment. Acta Metall. Mater. 42, 475 (1994).

26. E.C. Aifantis: Exploring the applicability of gradient elasticity to certain micro/nano reliability problems. Microsyst. Technol. 15, 109 (2009). 
27. K.E. Aifantis and J.R. Willis: Interfacial jump conditions in straingradient plasticity and relations of Hall-Petch type. In Proceedings of the Seventh U. S. National Congress of Applied Mechanics (June 24-26 Chania/Greece) (2004); p. 372.

28. K.E. Aifantis and J.R. Willis: The role of interfaces in enhancing the yield strength of composites and polycrystals. J. Mech. Phys. Solids 53, 1047 (2005).

29. H.C. Rogers: The influence of hydrogen on the yield point in Fe. Acta Metall. 4, 114117 (1956).

30. E.J. Song, D-W. Suh, and H.K.D.H. Bhadeshia: Theory for hydrogen desorption in ferritic steel. Comput. Mater. Sci. 39, 36-44 (2013).

31. B.N. Lucas: An Experimental Investigation of Creep and Viscoelastic Properties Using Depth Sensing Indentation Techniques, Materials Science and Engineering (University of Tennessee, Knoxville, 1997).

32. G.M. Pharr, J.H. Strader, and W.C. Oliver: Critical issues in making small-depth mechanical property measurements by nanoindentation with continuous stiffness measurement. J. Mater. Res. 24, 653-666 (2009).

33. B. Merle, V. Maier-Kiener, and G.M. Pharr: Influence of modulus-to-hardness ratio and harmonic parameters on continuous stiffness measurement during nanoindentation. Acta Mater. 134, 167-176 (2017).

34. E.G. Herbert, S.A. Hackney, N.J. Dudney, and P.S. Phani: Nanoindentation of high purity vapor deposited lithium films: The elastic modulus. J. Mater. Res. 33, 1335-1346 (2018).

35. E.G. Herbert, P. Sudharshan, and K.E. Johanns: Nanoindentation of viscoelastic solids: A critical assessment of experimental methods. Curr. Opin. Solid State Mater. Sci. 19, 334339 (2015). 
36. W.C. Oliver and G.M. Pharr: An improved technique for determining hardness and elastic modulus using load and displacement sensing indentation experiments. J. Mater. Res. 7, 1564-1583 (1992).

37. E.G. Herbert, S.A. Hackney, N.J. Dudney, V. Thole, and P. S. Phani: Nanoindentation of high purity vapor deposited lithium films: A mechanistic rationalization of diffusionmediated flow. J. Mater. Res. 33, 1347-1360 (2018).

38. L.E. Samuels: The nature of mechanically polished metal surfaces: The surface deformation produced by the abrasion and polishing of 70: 30 brass. J. Inst. Met. 85, 51 (1956).

39. A.J. Wilkinson and D. Randman: Determination of elastic strain fields and geometrically necessary dislocation distributions near nanoindents using electron back scatter diffraction. Philos. Mag. 90, 1159-1177 (2010).

40. T. Ruggles: Characterization of geometrically necessary dislocation content with EBSD-based continuum dislocation microscopy. ProQuest Dissertation and Theses, Brigham Young University, Provo, Utah, 4392, 2015.

41. T.P. Remington, C.J. Ruestes, E.M. Bringa, B.A. Remington, C. H. Lu, B. Kad, and M.A. Meyers: Plastic deformation in nanoindentation of tantalum: A new mechanism for prismatic loop formation. Acta Mater. 78, 378-393 (2014).

42. E. Nes: Recovery revisited. Acta Metall. Mater. 43, 2189 (1995).

43. T. Britton, D. Randman, and A. Wilkinson: Nanoindentation study of slip transfer phenomenon at grain boundaries. J. Mater. Res. 24, 607 (2009).

44. R.W. Armstrong, I. Codd, R.M. Douthwaite, and N.J. Petch: The plastic deformation of polycrystalline aggregates. Philos. Mag. 7, 4558 (1962). 
45. E.O. Hall: The deformation and ageing of mild steel: III discussion of results. Proc. Phys. Soc. B 64, 747 (1951).

46. N. Petch: The cleavage strength of polycrystals. J. Iron Steel Inst. 174, 25 (1953).

47. N. Hansen: The effect of grain size and strain on the tensile flow stress of aluminium at room temperature. Acta Metall. 25, 863 (1977).

48. L.P. Evers, D.M. Parks, W.A.M. Brekelmans, and M.G.D. Geers: Crystal plasticity model with enhanced hardening by geometrically necessary dislocation accumulation. J. Mech. Phys. Solids 50, 2403 (2002).

49. K. Wierzbanowski, A. Baczmanski, P. Lipinski, and A. Lodini: Arch. Metall. Mater. 52, 77 (2007).

50. A. Ma and A. Hartmaier: On the influence of isotropic and kinematic hardening caused by strain gradients on the deformation behaviour of polycrystals. Philos. Mag. 94, 125 (2014).

51. J.C.M. Li: Petch relation and grain boundary sources. Trans. Metall. Soc. AIME 227, 239 (1963).

52. J. Meakin and N.J. Petch: Report ASD-TDR-63-324, Symposium on the Role of Substructure in the Mechanical Behavior of Metals (ASD-7DR-63-324, Orlando, 1963); pp. 234-251.

53. M. Kato: Hall-Petch relationship and dislocation model for deformation of ultrafineGrained and nanocrystalline metals. Mater. Trans. 55, 19-24 (2014).

54. H. Conrad, S. Feuerstein, and L. Rice: Effects of grain size on the dislocation density and flow stress of niobium. Mater. Sci. Eng. 2, 157-168 (1967). 
55. H. Conrad: Effect of grain size on the lower yield and flow stress of iron and steel. Acta Metall. 11, 75 (1963).

56. U.F. Kocks: Metall. Trans. 1, 1123 (1970).

57. K. Takeda, N. Nakada, T. Tsuchiyama, and S. Takaki: Effect of interstitial elements on Hall-Petch coefficient of ferritic iron. ISIJ Int. 48, 1122 (2008).

58. X. Wei, A. Konstantinidis, C. Qi, and E. Aifantis: Gradient plasticity used for modeling extrinsic and intrinsic size effects in the torsion of Au microwires. J. Mech. Behav. Mater. 25,53 (2016).

59. A.G. Evans and J.W. Hutchinson: A critical assessment of theories of strain gradient plasticity. Acta Mater. 57, 1675 (2009).

60. X. Zhang and K.E. Aifantis: Examining the evolution of the internal length as a function of plastic strain. Mater. Sci. Eng., A 631, 27 (2015).

61. G. Feng and W.D. Nix: Indentation size effect in MgO. Scr. Mater. 51, 599 (2004).

62. K.E. Aifantis and J.R. Willis: Scale effects induced by straingradient plasticity and interfacial resistance in periodic and randomly heterogeneous media. Mech. Mater. 38, 702 (2006).

63. G. Sachs: Zur Ableitung einer Fließbedingung. Z. Ver. Deutscher Ing. 72, 734 (1928).

64. G. Mokios and E.C. Aifantis: Gradient effects in micro-/ nanoindentation. Mater. Sci. Technol. 28, 1072 (2012).

65. J.S. Stoelken and A.G. Evans: A microbend test method for measuring the plasticity length scale. Acta Mater. 46, 5109 (1998). 
66. C.F. Niordson and J.W. Hutchinson: Basic strain gradient plasticity theories with application to constrained film deformation. J. Mech. Mater. Struct. 6, 395 (2011).

67. R.A.B. Engelen: Plasticity-induced Damage in Metals: Nonlocal Modelling at Finite Strains (Technische Universiteit Eindhoven, Eindhoven, 2005). 


\section{Appendix 3.A. Linearization of the Taylor Hardening flow stress relation.}

This paper focusses on the correlation of ISE with indentation depth-dependent dislocation density through the Taylor hardening flow rule. Ashby [3] has suggested that the plastic (in) compatibility at the grain boundary may be correlated with an increase in density of GNDs there, then leading to an increase in hardening due to the Taylor hardening mechanism [38]. GNDs were correlated to the gradient in plastic strain by Ashby in 1970 [3] and as such was part of the origination of SGP theories, which proliferate the literature 50 years later. The continuum models of SGP do have a linearized form that have been used successfully to develop analytical approaches to aid in the physical interpretation of deformation phenomenon [49]. Given the focus on the Taylor hardening mechanism in this work, it is of interest and a necessity to relate the mechanistic Taylor dislocation hardening relation to this linear, relatively transparent continuum SGP analysis. The first step in such an analysis is to examine under what conditions the Taylor strengthening equation, upon which Eq. (3.1) - (3.8) are based, can be consistent with the linear continuum SGP flow rule. This particular issue relating Taylor flow theory with a continuum SGP model has been discussed previously [64], although using an approach different from that pursued here. We attempt here to relate an SGP linear flow stress model to the Taylor flow rule by relating the plastic strain and plastic strain gradient to the dislocation density as follows:

$$
\sigma(\mathrm{x})=\beta\left[\varepsilon_{p}+l\left(\frac{d}{d x} \varepsilon_{p}\right)\right] \cong \beta \mathrm{b} \sqrt{\rho_{S S D}(x)+\rho_{G N D}(x)}
$$

where $\sigma(\mathrm{x})$ is a position-dependent flow stress, $\beta$ is the hardening modulus, $\varepsilon_{p}$ is the plastic strain, 1 is a characteristic length, and $x$ is the position variable. Equation [3.A(1)] is rewritten by defining $\overline{\rho_{S S D}}$ as the volume averaged SSD dislocation density and $\Delta_{S S D}(\mathrm{x})$ as the variation in the SSD dislocation density about the average: 


$$
\sigma(\mathrm{x})=\beta \mathrm{b} \sqrt{\overline{\rho_{S S D}}+\Delta_{S S D}(x)+\rho_{G N D}(x)}
$$

Relating Eq. [3.A(2)] to indentation, $\rho_{S S D}$ is the average dislocation density for indentation in the grain interior and is not a function of position( $\mathrm{x}$ ). A pyramid, wedge, or spherical tip would develop position-dependent dislocation densities due to the indenter geometry [1]. The presence of a grain boundary in the indentation volume is also considered here to introduce spatial variations in dislocation density. Equation [3.A(2)] is approximated to Eq. [3.A(3)] through the sequential Taylor series on the smaller terms, $\Delta_{S S D}(\mathrm{x})$ and $\rho_{G N D}(\mathrm{x})$, and compared with the linear SGP flow stress rule in Eq. [3.A(1)]

$$
\beta\left[\varepsilon_{p}+l\left(\frac{d}{d x} \varepsilon_{p}\right)\right]=\beta \mathrm{b}\left\{\sqrt{\overline{\rho_{S S D}}}+\frac{1}{2 \sqrt{\overline{\rho_{S S D}}}}\left[\Delta_{S S D}(x)+\rho_{G N D}(x)\right]\right\}
$$

The comparison of the lhs and rhs of Eq. [3.A(3)] is carried out by noting the Orowan equation:

$$
\varepsilon_{p}=\rho b X=\rho b \frac{1}{\sqrt{\rho}} \sim\left[\sqrt{\overline{\rho_{S S D}}}+\frac{\Delta_{S S D}(x)}{2 \sqrt{\overline{\rho_{S S D}}}}\right]
$$

where $\mathrm{X}$ is the slip length, which can be taken as the dislocation spacing. To correlate the plastic strain as defined in Eq. [3.A(4)] with Eq. [3.A(1)], it is necessary to take the derivative of the plastic strain. Given Eq. [3.A(4)] and the well-known relation [3], 


$$
\frac{d}{d x} \varepsilon_{p}=b \rho_{G N D} \sim b \frac{d}{d x}\left[\frac{\Delta_{S S D}(x)}{2 \sqrt{\overline{\rho_{S S D}}}}\right]
$$

Then, it is possible to rewrite Eq. [3.A(1)] to first order in small terms using Eq. [3.A.(4)] and $[3 . \mathrm{A}(5)]$ :

$$
\sigma(\mathrm{x})=\beta\left[\varepsilon_{p}+l\left(\frac{d}{d x} \varepsilon_{p}\right)\right] \sim \beta \mathrm{b}\left[\sqrt{\overline{\rho_{S S D}}}+\frac{\Delta_{S S D}(x)}{2 \sqrt{\overline{\rho_{S S D}}}}+l \rho_{G N D}(x)\right]
$$

where 1 is defined through Eq. [3.A(3)] and [3.A(6)] as 1/2 the average dislocation spacing:

$$
l=\frac{1}{2 \sqrt{\overline{\rho_{S S D}}}}
$$

The error involved in the analysis leading to Eq. [3.A(6)] and [3.A(7)] may be determined by Eq. [3.A(8)]. It may be seen in Fig. 3.A(1) that the error is less than $6 \%$ when the averaged dislocation density $\overline{\rho_{S S D}}=\overline{\rho_{G N D}}=10^{18} \mathrm{~m}^{2}$. This sacrifice in precision is required for a transparent analysis of the observed phenomena. As support for the overall result developed in Eq. [3.A(1) - 3.A(7)], the definition of the length scale in Eq. [3.A(7)] is seen to be consistent with the conclusions of Evans and Hutchinson [59] obtained by attempting to relate the plastic dissipation term for the linear flow rule to the Taylor hardening expression. Physically, Eq. [3.A(6) and 3.A(7)] is describing the contribution that the "extra" dislocation from the spatial variation in plastic strain will make toward the total 
dislocation hardening, so that for small $l$, the contribution for a given deformation gradient to the total hardening will be limited. This is a direct consequence of the additive formulation for the different dislocation densities in Eq. [3.A(2)]. However, this physical description does not take into account that the deformation gradient magnitude may increase with a decrease in the slip length, $l$, depending on the boundary conditions.

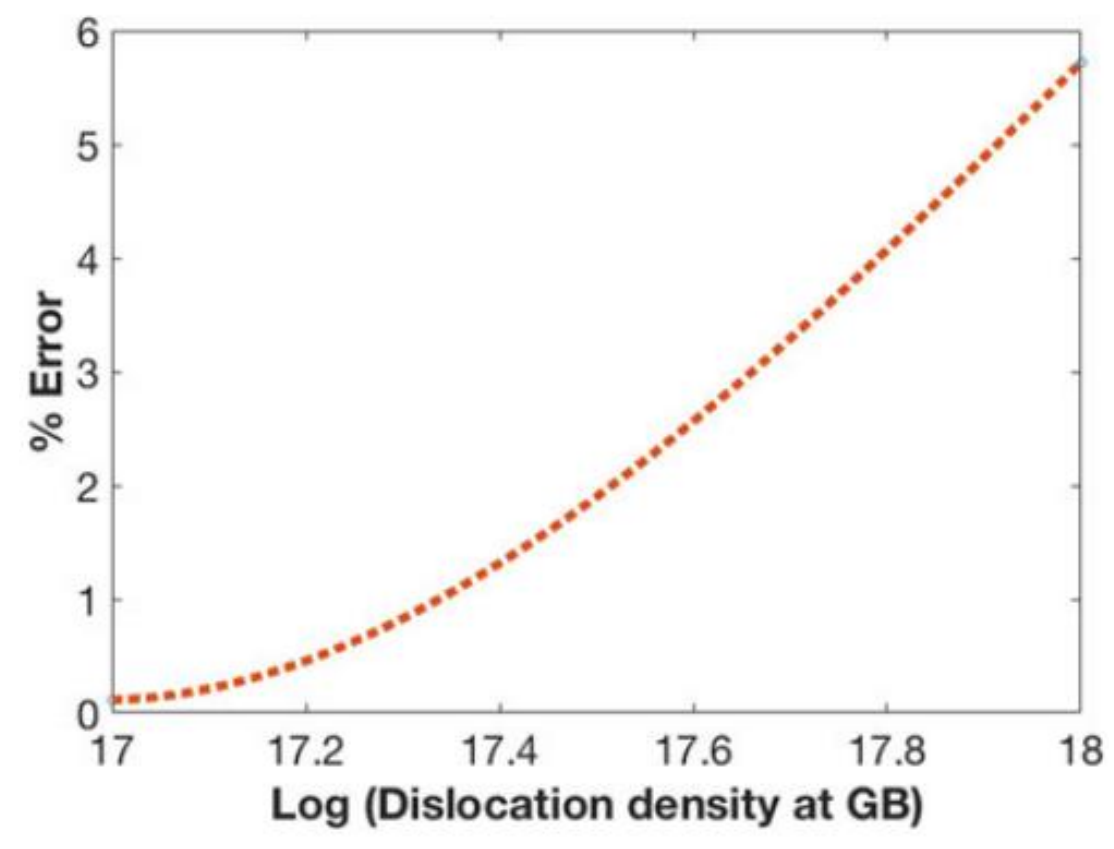

Figure 3.A.1. Percent error in Eq. (3.A(6)) when compared to Eq. (3.A(1)). The error is less than $1 \%$ when $\rho_{g b}=0.1 \rho_{A}$ and is $\sim 6 \%$ at $\rho_{g b}=\rho_{A}=10^{18} \mathrm{~m}^{-2}$.

$$
\% \text { Error }=\left(1-\frac{\sqrt{\overline{\rho_{S S D}}+\overline{\overline{\rho_{G N D}}}}}{\sqrt{\overline{\rho_{S S D}}}+\frac{\overline{\bar{a}_{G N D}}}{2 \sqrt{\bar{\rho}_{S S D}}}}\right) \times 100
$$


Note that the integration of $\Delta_{\mathrm{SSD}}(\mathrm{x})$ is required to be null, as this is the deviation about the average SSD dislocation density. This completes the analysis demonstrating under what conditions the Taylor hardening law is consistent with the linear SGP flow rule when the spatial variation of the dislocation density in general is less than or equal to the average indentation dislocation density. Connecting this analysis with Eq. (3.2) - (3.4) is of considerable interest as well. Using the sequential Taylor series again for Eq. (3.4a) gives

$$
H_{o} \sqrt{1+\frac{h^{*}+h_{g b}^{*}}{f^{3} h}} \cong H_{o}\left(1+\frac{h^{*}}{2 f^{3} h}+\frac{h_{g b}^{*}}{2 f^{3} h}\right)
$$

For intrinsic ISE, comparison of Eq. [3.A(9) and 3.A(7)] confirms the Evans et al. analysis [59] that $l \propto \frac{1}{\sqrt{\rho_{S S D}}}$ and that $h^{*} \propto \frac{1}{\left(b \overline{\rho_{S S D}}\right)}$ and that the two length scales are not equivalent. 


\section{Appendix 3.B. Consideration of the limiting case for the Schmid factor term, $\Delta \mathbf{m}$.}

The Schmid factor term, $\Delta \mathrm{m}=\frac{m_{1}-m_{1}}{m_{1} m_{2}}$, defined in Eq. (3.13) is difficult to analytically determine for Berkovich nanoindentation as the resulting stress tensor is multidimensional and, in particular, non-uniform. However, it is possible to consider a limiting case for an effective $\Delta \mathrm{m}$. There are statistically significant hardness differences between the grain surfaces [Table 3.B(1)], and we take the softest of the observed grain to have an effective Schmid factor of 0.5 . The hardness difference between the softest grain and the other grains is then attributed to a difference in the Berkovich effective Schmid factor through the relations Eq. (3.B(1)) and (3.B(2)). The analysis can be taken a step further by noting that there is a correlation between the adjacent grain hardness difference in Fig. 3.B(1) and the increase in grain boundary hardness relative to the average of the adjacent grain hardness or relative to the hardness of the hardest grain. Not only does this limiting case analysis provide a range of $\Delta \mathrm{m}$ values for examination of curve fitting results but also provides an additional qualitative test for the proposed concept that the hardening due to local indentation of the grain boundary is related to the Ashby plastic incompatibility argument. The results of these measurements are shown in Table 3.B(1). The limiting case values for $\Delta \mathrm{m}$ that result from the differences in grain hardness [from Eq. 3.B(2)] are shown in Fig. 3.B2(a). In Fig. 3.B2(b), the trend of an increase in grain boundary hardness with increasing $\Delta \mathrm{m}$ is demonstrated (relative to the average grain hardness).

$$
\begin{gathered}
H \propto \sigma \propto \frac{1}{m} \\
m_{\text {grain }(i)}=m_{\text {softest grain }} \times \frac{m_{\text {softest grain }}}{m_{\text {grain }(i)}}
\end{gathered}
$$


Figure 3.B(2) offers some support for the idea that plastic incompatibility at the grain boundary, as described by Ashby [3], is the root cause of the experimental observations in Fig. 3.7. According to the Ashby theory, the GND dislocation density and plastic strain should increase as $\Delta \mathrm{m}$ increases, and this is indeed the trend of increasing hardness with increasing $\Delta \mathrm{m}$ observed in Fig. 3.B2(b).

This range in $\Delta \mathrm{m}$ considered in Fig. 3.B(2) does allow for the further interpretation of Eq. (3.8) and subsequently Eq. (3.5) using the Ashby boundary condition, Eq. (3.10), $\gamma=\gamma_{1}-$ $\gamma_{2}=\varepsilon_{p} \times \Delta m$ such that

$$
\frac{\gamma}{b f\left(\frac{1-e^{-n}}{n}\right)}=\frac{\varepsilon_{p} \Delta \mathrm{m}}{b f\left(\frac{1-e^{-n}}{n}\right)}=4.5 \times 10^{8} \mathrm{~m}^{-1}
$$

Taking the usual characteristic strain for Berkovich indentation as $\varepsilon_{p} \sim 0.1, \Delta \mathrm{m} \sim 0.3$ from Fig. 3.B(2), and $\mathrm{f} \sim 2.0$ to 3.2 from the Eq. (3.5) Hall-Petch coefficient gives from Eq. $[3 . \mathrm{B}(3)]$ that $\mathrm{n} \sim 0.2$ to 0.45 . The relatively small value of $\mathrm{n}$ and $\mathrm{f}$ found here would suggest that the plastic zone due to the grain boundary deformation is localized at the grain boundary but relatively uniform, consistent with the measurements of Vachhani [20] and the analysis of Evers et al. [48]. It is also noted that this value of $f$ suggesting grain boundary plastic zone localization $[f \sim 1 / \tan (\theta)]$ would be specific to the Ashby mechanism caused by the local grain boundary indentation geometry described in this paper, as opposed to the value of $f$ from considerations of remote indentation extrinsic ISE [22] or considerations of the intrinsic ISE [61] as these other ISE are attributed to different deformation processes. 

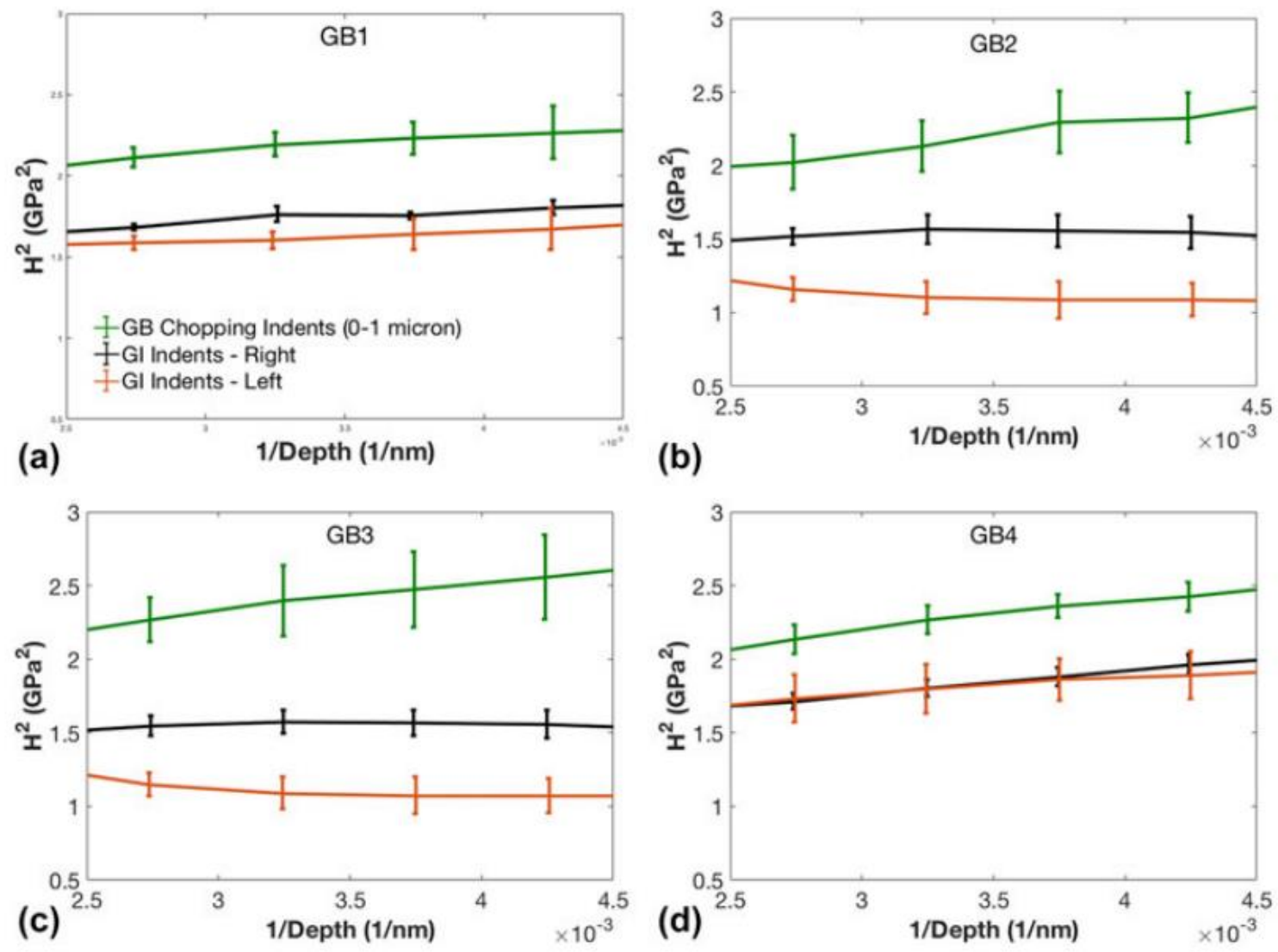

Figure 3.B.1. Representative Nix-Gao plots for comparing grain boundary chopping indentation (green) with adjacent grain interior (black and orange) for (a) GB1, (b) GB2, (c) GB3, (d) GB4. 

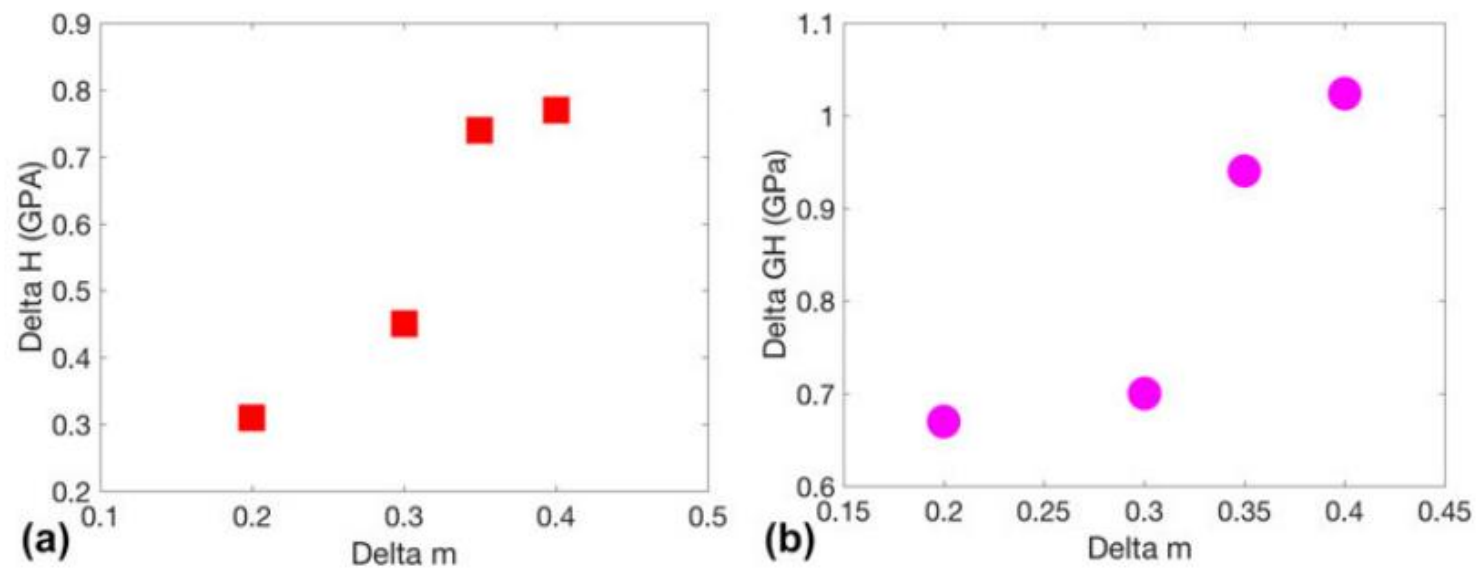

Figure 3.B.2. Correlating difference in hardness of adjacent grains (delta $\mathrm{H}$ ) with the limiting case for $(\Delta \mathrm{m})$ Eq. 3.B (2). (b) Correlating difference in hardness between grain boundary and harder adjacent grain interior (delta $\mathrm{GH})$ with estimated $(\Delta \mathrm{m})$.

\begin{tabular}{|c|c|c|c|}
\hline Grain boundary & m (right grain) & m (left grain) & $\boldsymbol{\Delta} \mathbf{m}=\frac{\boldsymbol{m}_{\mathbf{1}}-\boldsymbol{m}_{\mathbf{1}}}{\boldsymbol{m}_{\mathbf{1}} \boldsymbol{m}_{\mathbf{2}}}$ \\
\hline GB1 & 0.38 & 0.40 & 0.131 \\
\hline GB2 & 0.43 & 0.50 & 0.325 \\
\hline GB3 & 0.42 & 0.50 & 0.380 \\
\hline GB4 & 0.40 & 0.37 & 0.200 \\
\hline
\end{tabular}

Table 3.B.1. Limiting case analysis for the effective Schmid factor. 


\section{Appendix 3.C. Continuum SGP Approximation.}

The purpose of this appendix is to examine the sources of error associated with substitution of a proposed plastic strain field into an SGP flow rule to estimate the resulting equivalent stress. Certainly, the simple substitution approach has been done previously by other investigators, specifically Wei et al. [58] and Stoelken et al. [65]. It should also be noted even the more involved plastic potential minimization approach has been utilized by presupposing the general form of the displacement field, where the coefficients are then determined to satisfy the boundary conditions and to minimize the potential [66]. The formulation of the SGP continuum analysis adapted to the linear analysis pursued here was recently described by Wei et al. [58], who defined a general form for the continuum SGP equivalent stress as

$$
\sigma_{e}=\beta\left(\overline{\varepsilon_{p}}+l_{1}\left[\vec{\nabla} \overline{\varepsilon_{p}} \cdot \vec{\nabla} \overline{\varepsilon_{p}}\right]^{q}+l_{2} \nabla^{2} \overline{\varepsilon_{p}}\right)
$$

where we take the characteristic length $\mathrm{l}_{2}=0$ and the exponent $\mathrm{q}=1 / 2$, as represented by Eq. [3.A(1) and 3.A(3)]. The equivalent stress and plastic strain measures are defined as $\sigma_{e}=\sqrt{\left(\frac{3}{2}\right) s_{i j} s_{i j}}$ and $\overline{\varepsilon_{p}}=\sqrt{\left(\frac{3}{2}\right) \varepsilon_{i j}^{p} \varepsilon_{i j}^{p}}$, where $s_{i j}$ is the deviatoric stress component and $\varepsilon_{i j}^{p}$ is a component of the plastic stress tensor. The equivalent stress, $\sigma_{e}$, is then a function and the magnitude of the gradient squared $\left(\vec{\nabla} \overline{\varepsilon_{p}} \cdot \vec{\nabla} \overline{\varepsilon_{p}}\right)$ as utilized in Eq. (3.C(1)). Here, we will consider a two-dimensional, plastic strain field based on a displacement potential satisfying the biharmonic partial differential equation, so that $\left(\vec{\nabla} \overline{\varepsilon_{p}} \cdot \vec{\nabla} \overline{\varepsilon_{p}}\right)^{1 / 2}=$ $\left(\frac{1}{2}\right)\left(\left|\nabla_{x} \overline{\varepsilon_{p}}\right|+\left|\nabla_{y} \overline{\varepsilon_{p}}\right|\right)$. Thus, Eq. (3.C(1a)) can be rewritten as follows: 


$$
\sigma_{e}=\beta\left(\overline{\varepsilon_{p}}+\frac{l_{1}}{2}\left|\nabla_{x} \overline{\varepsilon_{p}}\right|+\left|\nabla_{y} \overline{\varepsilon_{p}}\right|\right)
$$

The simplest approach [58,65], but not the most accurate, would be to substitute a plastic strain field that satisfied all boundary, compatibility, and equilibrium conditions directly into Eq. [3.C(1b)] to determine the equivalent stress. The error in the simple approach arises from a neglect of the elastic strains, essentially assuming that all strains are plastic. The error here can be estimated by following [67], taking the general expression for an elastic-plastic solid in the plastic zone (small strain) as

$$
\varepsilon_{i j}=\frac{3}{2 E} \dot{S}_{i j}+\frac{3}{2} \dot{\varepsilon}_{p} \frac{s_{i j}}{\sigma_{e}}
$$

With $\varepsilon_{i j}$ as the total strain tensor. The boundary conditions of the displacements and strains due to the indentation of the grain boundary will be prescribed, from which the total strain tensor can be determined using the Marguerre displacement function with $v=1 / 2$ for plastic deformation. In this manner, the compatibility conditions relating displacement and strain will be satisfied, along with the conditions for stress component equilibrium. A compressive plastic displacement of magnitude $\mathrm{h}$ will be applied to the surface parallel the

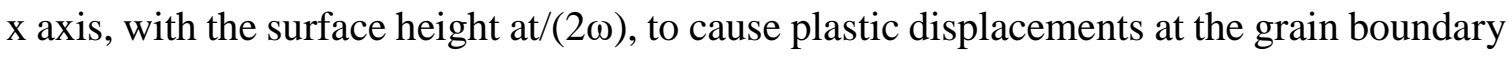
lying on the xz plane at $y=0$ in accordance with the Ashby model [Eq. (3.15)] [3]. The simplest, relevant displacement potential that will satisfy the boundary, compatibility, and equilibrium conditions is then proposed as:

$$
\Psi=A x^{2} y+B e^{-\omega y} \sin (\omega y)
$$


where $\mathrm{A}$ and $\mathrm{B}$ are integration constants and $\mathrm{x}$ is a spatial frequency, all to be determined by boundary conditions. The coefficient, A, in Eq. [3.C(3a)] can be considered to represent the intrinsic aspect of the indentation, while the coefficient, B, represents the extrinsic effect of the grain boundary. Equation [3.C(3a)] may be used to obtain an approximate value for the total strain tensor components, $\varepsilon_{i j}$, as

$$
\begin{gathered}
\varepsilon_{x x}=\frac{4}{3}\left(-\nabla_{x x y} \Psi+\frac{1}{2} \nabla_{y y y} \Psi\right) \\
\varepsilon_{y y}=\frac{4}{3}\left(\frac{5}{2} \nabla_{x x y} \Psi+\nabla_{y y y} \Psi\right) \\
\varepsilon_{x y}=\frac{4}{3}\left(\nabla_{x x x} \Psi+\frac{1}{2} \nabla_{x y y} \Psi\right)
\end{gathered}
$$

To zero order in $\mathrm{B} \omega^{3}$, the approximate relation $-2.5 \mathrm{~s}_{\mathrm{xx}} \sim \mathrm{s}_{\mathrm{yy}}$ will hold if the elastic (and plastic) strains are proportional to the total strains, giving $\sigma_{\mathrm{e}} \sim 3.3\left|\mathrm{~s}_{\mathrm{xx}}\right|$. This relation allows for the substitution of $\sigma_{\mathrm{e}}$ for $\mathrm{s}_{\mathrm{xx}}$ in Eq. (3.C(2)), which then gives the $\mathrm{x}$ total strain tensor component as

$$
-\varepsilon_{x x} \sim-\frac{1}{2}\left(\frac{\sigma_{e}}{E}+\varepsilon_{p}\right)
$$


Further substitution of Eq. (3.C(1b)) for re and Eq. (3.C(3b)) for $\varepsilon_{x x}$ in Eq. (3.C(4)) gives a linear, first-order partial differential equation similar to that presented in the appendices of Evans et al. [59] for lower order theory. The linear, first-order partial differential equation obtained from Eq. (3.C(4)) has a solution to zero order in $\left(\frac{\beta}{E}\right)$ as

$$
\overline{\varepsilon_{p}}=2\left|\varepsilon_{x x}\right|+C_{1} f(x, y)
$$

where $\mathrm{C} 1$ is an integration constant and the function $\mathrm{f}(\mathrm{x}, \mathrm{y})$ exponentially decays in the $\mathrm{x}$ direction with length scale, 1 . This integration constant, $\mathrm{C}_{1}$, could be used to "correct" any nonphysical boundary condition resulting for the higher order (gradient induced) stress, such as setting free surface tractions resulting from gradient terms to null value. However, the proposed model has no free surfaces; therefore, $\mathrm{C}_{1}$ is taken to be zero. We also note the full solution to Eq. (3.C(1)) is of particular interest, because it introduces a mathematically rich environment for physical analyses, but the full solution not considered further in this paper. Simply put, the Eq. (3.C(1)) solution with relative error $\left[1-\frac{1}{1+\frac{\beta}{E}+\frac{\Delta m H_{0}}{\beta}}\right]$ gives the plastic strain measure as proportional to the $\mathrm{x}$-component of the imposed total strain tensor components. As such,

$$
\overline{\varepsilon_{p}}=4 A+3 B \omega^{3} \sin (\omega x) \exp (-\omega y)
$$

This is essentially the same simplified approach utilized by Stoelken et al. [65] and Engelen [67], where the plastic strain measure is proportional to a component of the total strain 
tensor. The solution for the individual strain tensor components is considered for $\mathrm{y}>0$. The solution for $\mathrm{y}<0$ could also be written in the form of Eq. (3.C(2)) and, thus, mirror the $y>0$ solution with a change in sign of the exponential argument. This interpretation of the solution could allow the normal components of plastic strain to be continuous, but not differentiable, across the grain boundary.

The variables A, B, and $\omega$ can be determined from the boundary conditions expressed by Eq. [3.C(7a) - 3.C(7c)]. Equation (3.C(7a)) is the rendition of Eq. (3.15) for the twodimensional plastic strain measure, where the subscript $B=0$ on the lhs refers to the plastic strain measure that would occur without the grain boundary present:

$$
\overline{\varepsilon_{p, B=0}} \frac{\Delta m}{L}=\left.\nabla \overline{\varepsilon_{p}}\right|_{y=0}
$$

and the trigonometric terms in Eq. (3.C(7a) are averaged over the length $\pi /(2 \omega)$. Equation (3.C(7b)) specifies that the plastic displacement at the surface is controlled by the geometry of the indenter, which is uniform in this simple model. Although simplistic in presentation, this boundary condition results in the sine function dependence for $\psi$ in Eq. (3.C(3a)); thus, there is no $y$ dependence on the plastic displacement along the surface $(x=\pi /(2 \omega)$ due to the grain boundary:

$$
\left.u_{x}\right|_{x=\pi / 2 \omega}=-h
$$


The boundary condition in Eq. (3.C(7c)) guarantees that $\mathrm{H}$ will not show a grain boundary or a size effect when the grain boundary is not present $(\Delta \mathrm{m}=0)$ and/or h becomes very large:

$$
\left.\beta \overline{\varepsilon_{p}}\right|_{x=\frac{\pi}{2 \omega}, \Delta m=0}=H_{o}
$$

where the exponential terms in Eq. (3.C(7c)) are averaged over the length $\pi /(2 \omega)$.

The stress contribution from the grain boundary due to the averaged equivalent stress at the surface is then obtained from Eq. [3.C(1) - (3.C(7)] by averaging the exponential $y$ dependent term over the length $\pi /(2 \omega)$ to give the stress contribution due to the coefficient 3.B and the extrinsic ISE as

$$
\left[\left.\overline{\sigma_{g b}}\right|_{x=\frac{\pi}{2 \omega}}\right]_{\text {extrinsic }}=\left(\frac{0.12 n H_{o} l_{1} \Delta \mathrm{m}}{h}\right)+0.02 n \beta \Delta \mathrm{m}
$$

where $\mathrm{L}=\mathrm{h} / \mathrm{n}$. Equation (3.C(8)) has an inverse $\mathrm{h}$ dependence and, thus, describes the extrinsic ISE. Drawing from the concepts leading to Eq. (3.A(8)), the total stress from the addition of the intrinsic and extrinsic components of the deformation is then used to estimate $\mathrm{H}$ :

$$
H=\left[H_{o}\left(1+\frac{h^{*}}{2 f^{3} h}\right)\right]_{\text {intrinsic }}+\left[\left.\overline{\sigma_{g b}}\right|_{x=\frac{\pi}{2 \omega}}\right]_{\text {extrinsic }}
$$


As a final examination of physical validity, we follow the transparent (but highly approximate) demonstration of strain gradient effects in Ref. [59]. To develop this physical picture of the Ashby mechanism within the context of SGP, Eq. (3.12) - (3.15) are examined in terms the plastic strain and plastic strain gradient, which develops on the grain boundary due to the indentation process. From Eq. (3.12),

$$
\varepsilon_{p(g b)}=\frac{u_{(g b)}}{h}=\left(\frac{H_{O}}{\beta}\right) \Delta m
$$

And Eq. (3.12) with Eq. (3.15) is rewritten as follows:

$$
\frac{d}{d x} \varepsilon_{p(g b)}=\frac{u_{(g b)}}{h^{2}}=\left(\frac{H_{o}}{\beta h}\right) \Delta m
$$

For a one-dimensional gradient plasticity, linear flow rule, Eq. (3.A(1)), Eq. (3.C(10)), and (3.C(11)) may be rewritten as an estimate of the contribution of the grain boundary to the equivalent stress

$$
\sigma_{p(g b)}=\beta \Delta m\left(\frac{H_{O}}{\beta}+l \frac{H_{o}}{\beta h}\right)
$$

which has the same general form as Eq. (3.C(8)). Finally, writing Eq. (3.C(12)) in the form of Eq. [3.A(1) - 3.A(7)] for the Taylor flow stress relates the mechanical behavior to the dislocation density. 


$$
\sigma_{p(g b)}=\beta b \Delta m\left(\frac{H_{O}}{\beta b}+\frac{\beta b}{2 H_{o}} \frac{H_{o}}{\beta b^{2} h}\right)=\beta b\left(\sqrt{\overline{\rho_{g b}}}+\frac{\overline{\rho_{G N D(g b)}}}{2 \sqrt{\rho_{S S D}}}\right)
$$




\section{Appendix 3.D. Reprint Copywrite Permission}

SPRINGER NATURE LICENSE

TERMS AND CONDITIONS

Mar 05, 2021

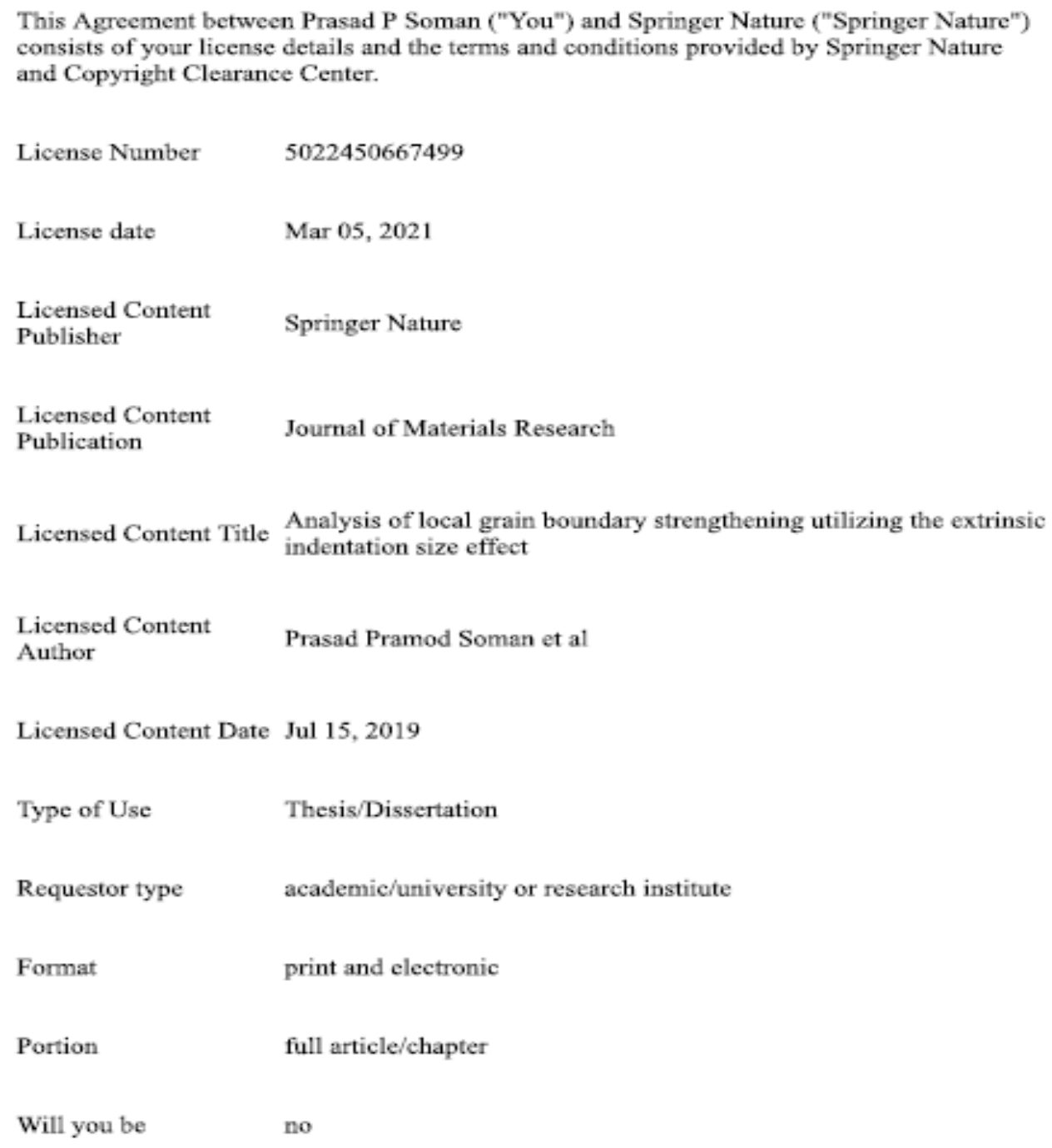


translating?

Circulation/distribution 50000 or greater

Author of this Springer yes
Nature content

Title

The Resolution of Grain Boundary Strengthening Mechanisms by Nanoindentation Induced Local Mechanical Response

Institution name Michigan Technological University

Expected presentation Apr 2021

date

Prasad P Soman

105, East State Street, Apt 1

Requestor Location

WEST LAFAYETTE, IN 47906

United States

Attn: Vaidehi Kale/Prasad P Soman

Total

0.00 USD

Terms and Conditions

\section{Springer Nature Customer Service Centre GmbH Terms and Conditions}

This agreement sets out the terms and conditions of the licence (the Lieence) between you and Springer Nature Customer Service Centre GmbH (the Licensor). By clicking "accept" and completing the transaction for the material (Licensed Material), you also confirm your acceptance of these terms and conditions.

\section{Grant of License}

1. 1. The Licensor grants you a personal, non-exclusive, non-transferable, world-wide licence to reproduce the Licensed Material for the purpose specified in your order only. Licences are granted for the specific use requested in the order and for no other use, subject to the conditions below.

1. 2. The Licensor warrants that it has, to the best of its knowledge, the rights to license reuse of the Licensed Material. However, you should ensure that the material you are requesting is original to the Licensor and does not carry the copyright of 
another entity (as credited in the published version).

1. 3. If the credit line on any part of the material you have requested indicates that it was reprinted or adapted with permission from another source, then you should also seek permission from that source to reuse the material.

\section{Scope of Licence}

2. 1. You may only use the Licensed Content in the manner and to the extent permitted by these Ts\&Cs and any applicable laws.

2. 2. A separate licence may be required for any additional use of the Licensed Material, e.g. where a licence has been purchased for print only use, separate permission must be obtained for electronic re-use. Similarly, a licence is only valid in the language selected and does not apply for editions in other languages unless additional translation rights have been granted separately in the licence. Any content owned by third parties are expressly excluded from the licence.

2. 3. Similarly, rights for additional components such as custom editions and derivatives require additional permission and may be subject to an additional fee. Please apply to Ioumalpermissions a springernature com/bookpermissions? springernature com for these rights.

2. 4. Where permission has been granted free of charge for material in print, permission may also be granted for any electronic version of that work, provided that the material is incidental to your work as a whole and that the electronic version is essentially equivalent to, or substitutes for, the print version.

2. 5. An alternative scope of licence may apply to signatories of the STM Permissions Guidelines, as amended from time to time.

\section{Duration of Licence}

3. 1. A licence for is valid from the date of purchase ('Licence Date') at the end of the relevant period in the below table:

\begin{tabular}{|l|l|}
\hline Scope of Licence & Duration of Licence \\
\hline Post on a website & 12 months \\
\hline Presentations & 12 months \\
\hline \hline Books and journals & Lifetime of the edition in the language purchased \\
\hline
\end{tabular}

\section{Acknowledgement}

4. 1. The Licensor's permission must be acknowledged next to the Licenced Material in print. In electronic form, this acknowledgement must be visible at the same time as the figures/tables/illustrations or abstract, and must be hyperlinked to the journal/book's homepage. Our required acknowledgement format is in the Appendix below. 


\section{Restrictions on use}

5. 1. Use of the Licensed Material may be permitted for incidental promotional use and minor editing privileges e.g. minor adaptations of single figures, changes of format, colour and/or style where the adaptation is credited as set out in Appendix 1 below. Any other changes including but not limited to, cropping, adapting, omitting material that affect the meaning, intention or moral rights of the author are strictly prohibited.

5. 2. You must not use any Licensed Material as part of any design or trademark.

5. 3. Licensed Material may be used in Open Access Publications (OAP) before publication by Springer Nature, but any Licensed Material must be removed from OAP sites prior to final publication.

\section{Ownership of Rights}

6. 1. Licensed Material remains the property of either Licensor or the relevant third party and any rights not explicitly granted herein are expressly reserved.

\section{Warranty}

IN NO EVENT SHALL LICENSOR BE LIABLE TO YOU OR ANY OTHER PARTY OR ANY OTHER PERSON OR FOR ANY SPECIAL, CONSEQUENTIAL, INCIDENTAL OR INDIRECT DAMAGES, HOWEVER CAUSED, ARISING OUT OF OR IN CONNECTION WITH THE DOWNLOADING, VIEWING OR USE OF THE MATERIALS REGARDLESS OF THE FORM OF ACTION, WHETHER FOR BREACH OF CONTRACT, BREACH OF WARRANTY, TORT, NEGLIGENCE, INFRINGEMENT OR OTHERWISE (INCLUDING, WITHOUT LIMITATION, DAMAGES BASED ON LOSS OF PROFITS, DATA, FILES, USE, BUSINESS OPPORTUNITY OR CLAIMS OF THIRD PARTIES), AND WHETHER OR NOT THE PARTY HAS BEEN ADVISED OF THE POSSIBILITY OF SUCH DAMAGES. THIS LIMITATION SHALL APPLY NOTWITHSTANDING ANY FAILURE OF ESSENTIAL PURPOSE OF ANY LIMITED REMEDY PROVIDED HEREIN.

\section{Limitations}

8. 1. BOOKS ONLYWhere 'reuse in a dissertation/thesis' has been selected the following terms apply: Print rights of the final author's accepted manuscript (for clarity, NOT the published version) for up to 100 copies, electronic rights for use only on a personal website or institutional repository as defined by the Sherpa guideline (www sherpia.ac,uk/momeon).

8. 2. For content reuse requests that qualify for permission under the STM Permissions Guidelines, which may be updated from time to time, the STM Permissions Guidelines supersede the terms and conditions contained in this licence. 


\section{Termination and Cancellation}

9. 1. Licences will expire after the period shown in Clause 3 (above).

9. 2. Licensee reserves the right to terminate the Licence in the event that payment is not received in full or if there has been a breach of this agreement by you.

\section{Appendix 1 - Acknowledgements:}

For Journal Content:

Reprinted by permission from [the Licensor]: [Journal Publisher (e.g.

Nature/Springer/Palgrave)] [JOURNAL NAME] [REFERENCE CITATION

(Article name, Author(s) Name), [COPYRIGHT] (year of publication)

For Advance Online Publication papers:

Reprinted by permission from [the Licensor]: [Journal Publisher (e.g. Nature/Springer/Palgrave)] [JOURNAL NAME] [REFERENCE CITATION

(Article name, Author(s) Name), [COPYRIGHT] (year of publication), advance online publication, day month year (doi: 10.1038/sj.[JOURNAL ACRONYM].)

For Adaptations/Translations:

Adapted/Translated by permission from [the Licensor]: [Journal Publisher (e.g. Nature/Springer/Palgrave)] [JOURNAL NAME] [REFERENCE CITATION

(Article name, Author(s) Name), [COPYRIGHT] (year of publication)

Note: For any republication from the British Journal of Cancer, the following credit line style anplies:

Reprinted/adapted/translated by permission from [the Licensor]: on behalf of Cancer Research UK: : [Journal Publisher (e.g. Nature/Springer/Palgrave)] [JOURNAL NAME] [REFERENCE CITATION (Article name, Author(s) Name).

[COPYRIGHT] (year of publication)

For Advance Online Publication papers:

Reprinted by permission from The [the Licensor]: on behalf of Cancer Research UK: [Journal Publisher (e.g. Nature/Springer/Palgrave)] [JOURNAL NAME]

[REFERENCE CITATION (Article name, Author(s) Name), [COPYRIGHT] (year of publication), advance online publication, day month year (doi: 10.1038/sj.

[JOURNAL ACRONYM])

For Book content:

Reprinted/adapted by permission from [the Licensor]: [Book Publisher (c.g. Palgrave Macmillan, Springer etc) [Book Title] by [Book author(s)]

[COPYRIGHT] (year of publication)

Other Conditions:

Version 1.3 
Questions? customercare $a$ copyright.com or +1-855-239-3415 (toll free in the US) or +1-978-646-2777. 


\section{CHAPTER 4: INDENTATION STRAIN RATE STUDY OF GRAIN BOUNDARY CHEMISTRY EFFECTS}

To be submitted for publication

\subsection{ABSTRACT:}

The indentation strain rate dependent hardness was utilized to interrogate hypotheses associated with the grain boundary strengthening through indentation-based hardness relaxation tests performed using two unique indentation geometries in pure iron and carbon doped iron. Grain boundary Indentations were performed at different strain rates on 4 specific boundaries with and without carbon, using remote and local indentation geometries, to understand a role of solute segregation in local plastic deformation at the grain boundary. The physical models, presented by Schoeck \& Gibbs and Evans \& Kocks used in conjunction with the Johnson-Cook parametric model facilitated determination of physical parameters such as activation energy, activation volume, athermal stress (or internal stress), as they relate to the grain boundary hardening mechanism. It was shown that in case of remote indentations, as proposed by the pile-up theory, solute segregation plays a critical role in enhancing the grain boundary strength against indentation strain whereas in case of local GB indentation the carbon segregation effect on hardness was statistically insignificant. The lack of a grain boundary chemistry effect for local deformation is rationalized through the Taylor-Ashby model, which is based on grain boundary crystallography (geometry) which is unaffected by carbon doping.

Further modified Johnson-Cook approach is presented to probe grain boundary pop-in events. The approach enables examination of the grain boundary chemistry effects through determination of back-stress variation on the onset of displacement burst event. The results suggested that the kinetics of grain boundary pop-in was influenced by the 
presence of carbon segregation at the grain boundary manifested through higher drop in athermal stress associated with carbon doped grain boundary.

\subsection{INTRODUCTION}

\subsubsection{Background:}

It was the Hall-Petch theory [1-2] that related strength of materials with the grain size through following equation:

$$
\sigma=\sigma_{o}+k \cdot d^{-\frac{1}{2}}
$$

Where, $\sigma_{o}$ - friction stress, $k$ - Hall-Petch coefficient, $d$-grain size or grain diameter

According to this theory, materials become stronger as their grain size decreases. This observation based on the experimental evidences can be extrapolated to suggest that grain boundary appears to play a key role in strength of metals and alloys.

In the decades following the proposal of the Hall-Petch strength-grain size relationship, research was focused on development of various strengthening mechanisms and models to explain contribution of grain boundary in materials strengthening. Some of the notable works include:

1. Hall-Petch dislocation pile-up model $[1,2]$

2. Taylor-Ashby strain gradient plasticity or work hardening model [3]

3. Grain boundary layer theory [4]

4. Grain boundary source theory $[5,6]$

While some of these models are highly accepted, all of them had a limited success wherein not all of the experimental observations could be explained with the help of a single theory or model. Some of the challenges presented in these theories are noted below: 
1. Although dislocation pile up at the grain boundary forms a foundation of Hall-Petch dislocation pile up model or grain boundary source model presented by $\mathrm{Li}$, there is no success in experimental observation of such dislocation pile-up.

2. Some of these models don't consider effect of strain rates on the grain boundary mechanical properties.

But the most common and a key drawback of all these models is:

3. An inadequacy in explaining a role of solute segregation during deformation process. The most accepted ideas related to the grain boundary strengthening emphasis on factors such as mismatch of slip geometry or plastic displacement inhomogeneity across the grain boundary [1, 7-9]. However, alloying does not obviously affect the slip geometry across the grain boundary.

The Hall-Petch relationship has been studied for wide ranging metals and alloys, traditionally using bulk testing approach, with alloys such as Mild steel, CopperAluminum, Copper-Zinc-Nickel systems [10-13] all showing large increase in the HallPetch slope as compared to pure metals. This has also been shown to be the case for application of recently developed nanoindentation based approaches (Iron-Carbon, IronNitrogen, Iron-Phosphorous) [14-18]. From all of these studies, it can be noted that the Hall-Petch coefficient is strongly influenced by the alloying element concentration (tabulated in table 4.1 and table 4.2). The most notable example is of pure iron and carbon doped iron, where a very small quantity of carbon doping appears to influence Hall-Petch coefficient of the Fe-C alloy indicating associated increase in strength of the material. It was Armstrong who first compiled various reports related to the role of alloying element effects on Hall-Petch coefficients, who also postulated that the grain boundary segregation results in solute pinning of dislocations in the grain boundary vicinity.

The objective of the work presented in this chapter is to study grain boundary strengthening associated with the carbon doping in pure iron using nanoindentations. 


\begin{tabular}{|c|c|}
\hline Metal/Alloy & K $\left(\mathbf{M p a . u m}^{-1 / 2}\right)$ \\
\hline $\mathrm{Fe}$ & 114 \\
\hline $\mathrm{Fe}-(30 \mathrm{ppm}) \mathrm{C}$ & 358 \\
\hline $\mathrm{Fe}-(60 \mathrm{ppm}) \mathrm{C}$ & 516 \\
\hline $\mathrm{Fe}-(30 \mathrm{ppm}) \mathrm{N}$ & 220 \\
\hline $\mathrm{Fe}-(60 \mathrm{ppm}) \mathrm{N}$ & 212 \\
\hline $\mathrm{P}(0)-\mathrm{Steel}$ & 150 \\
\hline $\mathrm{P}(1000)-$ Steel & 100 \\
\hline $\mathrm{C}(60)$ Steel & 550 \\
\hline $\mathrm{C}(60)-\mathrm{P}(1000)$ Steel & 270 \\
\hline
\end{tabular}

Table 4.1. Compilation of Hall-Petch coefficients reported for pure metals and alloys, reported in literature based on nanoindentation testing approach. [14-18]

\begin{tabular}{|c|c|}
\hline Metal/alloy & K $\left(M N . m^{-3 / 2}\right)$ \\
\hline Mild Steel $0.06 \%$ C & 0.66 \\
\hline Mild Steel $0.12 \%$ C & 0.74 \\
\hline Mild Steel $0.15 \%$ C & 0.79 \\
\hline Copper & 0.12 \\
\hline $\mathrm{Cu}-4$ at. $\% \mathrm{Al}$ & 0.23 \\
\hline $\mathrm{Cu}-8$ at. $\% \mathrm{Al}$ & 0.43 \\
\hline $\mathrm{Cu}-20$ at. $\% \mathrm{Zn}$ & 0.24 \\
\hline $\mathrm{Cu}-30$ at. $\% \mathrm{Zn}$ & 0.31 \\
\hline $\mathrm{Cu}-12.5 \mathrm{Ni}-12.5 \mathrm{Zn}$ & 0.26 \\
\hline $\mathrm{Cu}-29 \mathrm{Ni}-29 \mathrm{Zn}$ & 0.58 \\
\hline
\end{tabular}


Table 4.2. Compilation of Hall-Petch coefficients reported for pure metals and alloys, reported in literature based on bulk sample testing approach. [10-13]

technique. Although there have been many studies comparing tensile test results and/ or nano-indentation of grain boundaries in pure Fe and mild steel [10-18], this study offers the novel approach of comparing indentation geometry, and strain rate hardening of specific grain boundaries with and without carbon doping.

Unraveling the role of grain boundary in strengthening mechanism has long been an attractive and challenging topic of research for the materials engineering community committed to utilizing micron scale mechanisms to interpret macroscopic mechanical behavior. The development of nano-indentation technique has opened an avenue to further overcome challenges of interpretating bulk sample tests, such as tensile tests, using micromechanical mechanisms. Bulk mechanical testing of polycrystalline samples evaluates the contributions of many grain boundaries at the same time. Nanoindentation deforms a relatively small volume of material and can thus isolate and evaluate contribution of various individual microstructural features by comparing the local mechanical response of grain interior vs grain boundary, pure vs doped grain boundary and by the analysis of the local indentation strain rate effects. Although there have been many many recent nanoindentation based studies focused on the local mechanical behavior of grain boundaries [19-29], the finding presented here offer some originality in that specific grain boundaries are studied with and without solute additions, with two different indentations geometries and including the grain boundary strain rate - hardness response. The strain rate-hardness relationship is analyzed during constant load hardness relaxation experiments augmented by the analysis of hardness variations with different constant loading rates. The approach pursued in this paper seeks to examine the hypothesis of localized strengthening of the grain boundary by element segregation using the local deformation technique of nano-indentation. 
This work utilizes two specific indentation geometries, as depicted in the figure (4.1). A description of the indentation geometry relative to the grain boundary, termed 'local' and remote' has been introduced in chapter 3 [30]. The mechanical response of the of the local geometry was proposed to correspond to the Ashby theory of grain boundary hardening, while the remote geometry was suggested to exhibit a mechanical response consistent with the Hall-Petch pile-up mechanism of initial yielding at the grain boundary. It is important to note that the Johnson-Cook analysis presented in the result and discussion section for the grain boundary indentations is specific to the remote indentation geometry. The differences in grain boundary hardening behavior in the local and remote indentation are addressed in chapter 3 and in the result and discussion section of this chapter. In the result and discussion section a particular emphasis is given on the carbon doping effects (or lack thereof) for the case of local indentation.

The literature on grain boundary indentation studies has mostly utilized the remote indentation geometry to study local grain boundary hardening relative to its adjacent grain interior. Most of these reports utilize presence or absence of the displacement burst events observed during indentation loading stage, commonly referred to as pop-in events, to probe grain boundary yielding phenomenon. When considering pop-in events, it is important to note that there are reports of two distinct types of pop-in events reported in the literature $[21,28,29,31]$. During nanoindentation experiments in pure metals and alloys with defect free surfaces, first of such pop-in is observed at relatively shallow indentation depths $(<50$ $\mathrm{nm}$ ) and small applied load. These initial pop-ins are related to the dislocation nucleation as materials transitions from purely elastic to elastic-plastic deformation $[7,21,23,28,29$, 31-34]. These pop-ins should not be confused with the grain boundary pop-in events, that are observed only during nanoindentation experiments in the close vicinity of the grain boundary. While research groups that have investigated the grain boundary pop-in events propose different factors responsible for these events such as dislocation density, surface roughness, solute segregation at the grain boundary, it is pre-dominantly believed to be associated with the presence of interstitials 


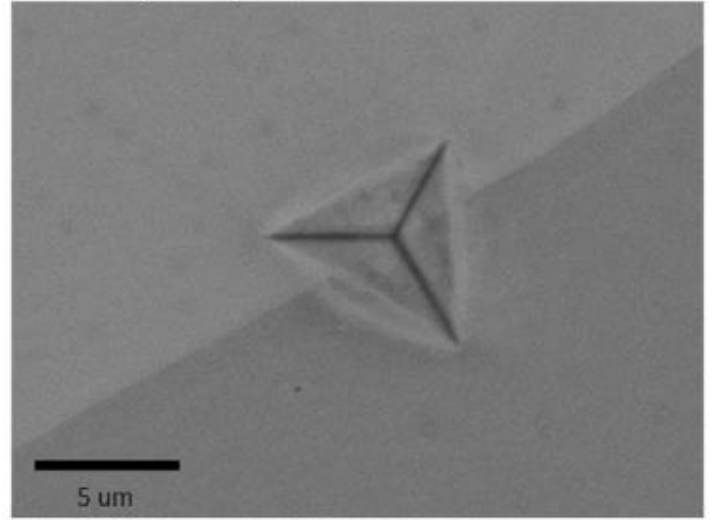

(a)

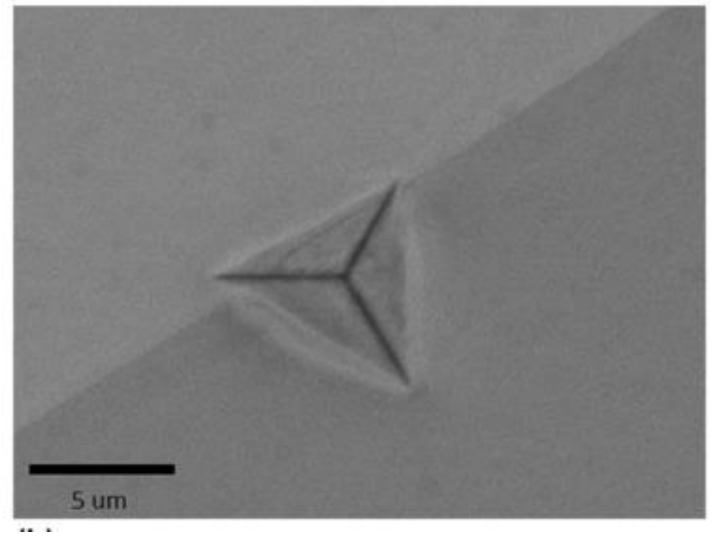

(b)

Figure 4.1. Backscatter channeling contrast image of (a) Local grain boundary indentation: the indenter tip is positioned less than 0.5 micron from the grain boundary with the "wedge" of indenter being perpendicular to the grain boundary plane, (b) Remote grain boundary indentation: the indenter tip is positioned $\sim 2$ micron from the grain boundary and the flat face of the indenter parallel to the boundary. [30]

elements in the vicinity of the grain boundary [29, 35]. Despite that, it is also important to acknowledge that the grain boundary pop-in events are not limited to alloys but are also observed in nanoindentation grain boundary studies on pure metals, as in this study.

While nanoindentation community has frequently utilized the ability to isolate and probe local mechanical responses of grain boundaries in pure and doped conditions, the previous studies have mainly focused on the analysis of the grain boundary pop-in events. The examination of the hardness and the distance of the indentation from the grain boundary at the point of the pop-in has limited these works to a measurement the grain boundary 'yield point'. In this paper, firstly authors acknowledge presence of grain boundary pop-in events in pure iron as well as carbon doped iron. But as mentioned previously, to investigate physical mechanism of grain boundary hardening this work 
primarily focuses on indentation stress relaxation tests at constant load performed at the grain boundary and adjacent grain interior, in pure and carbon doped conditions.

\subsubsection{Indentation Strain-Rate Hardness Analysis:}

The strain rate analysis and stress relaxation tests, using a bulk testing approach, are widely used to evaluate parameters (such as activation volume, athermal stress) associated with the plastic deformation in metals and alloys, by studying applied stressstrain rate responses [36-42]. While such approach is well established in the metals/alloys research and manufacturing world, authors note that utilization of indentation hardness relaxation tests to study local grain boundary strengthening is attempted for the first time through this work. In this work, the difference in the local and remote indentation is reexamined through the Johnson-Cook (JC) model interpretation of the constant load hardness relaxation data with particular emphasis on the grain boundary chemistry affected by carbon doping. The indentation experiments performed at the grain boundary and adjacent grain interior of pure iron and carbon doped iron, are governed by the load-time history, shown in figure (4.2).

The stress- strain rate responses from the stress relaxation tests are studies using various models, such as Johnson-Cook model [43], MTS model [44], Zerilli-Armstrong flow stress model [45]. All these models are parametric in nature and involve different mathematical complexity. The Johnson-Cook model is a simplest mathematical model and also widely adopted to study plastic deformation responses. This model is originally presented as: 


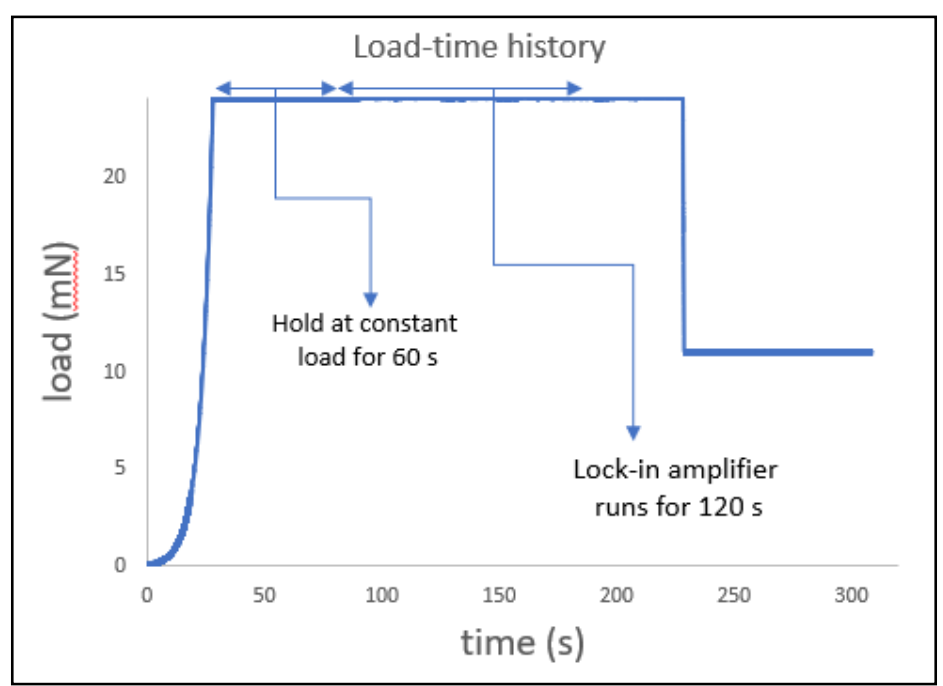

Figure 4.2. Load-time history used for the nanoindentation experiments.

$$
\sigma_{y}=\left[A+B\left(\varepsilon_{p}\right)^{n}\right]\left[1+C \ln \left(\frac{\dot{\varepsilon_{p}}}{\dot{\varepsilon}_{o}}\right)\right]\left[1-\left(\frac{T-T_{o}}{T_{m}-T_{o}}\right)\right]
$$

To model the stress - strain rate response during nanoindentation stress relaxation using the Johnson-Cook model, the hardness relaxation process in the constant load segment produces a correlation between the stress and strain rate, as shown in the equation (4.3(a)). From the room temperature indentation testing, (zero order in $\frac{T}{T_{m}}$ ) the time dependent hardness $\left(\sigma_{t}\right)$ within the iron hold segment may be interpreted by the JohnsonCook strain rate sensitivity model, given as:

$$
\left(\sigma_{t}\right)=\left(\sigma_{o}+\beta \varepsilon_{p}\right)\left(1+C \ln \left(\frac{\dot{\varepsilon_{p}}}{\dot{\varepsilon}_{o}}\right)\right)
$$


Where $\sigma_{y}$ is the yield stress, $\varepsilon_{p}$ is the plastic strain and $\beta$ is the hardening modulus. The term $\left(\sigma_{y}+\beta \varepsilon_{p}\right)$ is the static stress, $\dot{\varepsilon_{p}}$ is the plastic strain rate and $C$ and $\dot{\varepsilon_{o}}$ have been considered to be material constants. However, within the application of this model to the nano-indentation results in this work, the $C$ and $\dot{\varepsilon_{o}}$ are found to depend on the local microstructure and chemistry.

By rearranging equation (4.3(a)):

$$
\dot{\varepsilon_{p}}=\dot{\varepsilon_{o}} * \exp \left[\frac{\sigma(t)-\left(\sigma_{o}+\beta \varepsilon_{p}\right)}{C\left(\sigma_{o}+\beta \varepsilon_{p}\right)}\right]
$$

The term $\sigma_{y}+\beta \varepsilon_{p}$ has generally been considered to be the 'athermal stress', often associated with internal stresses of defect interaction with length scale dependence greater than the atomic length scale, such as Taylor hardening and dislocation pile ups.

The interpretation of the Berkovich indentation strain rate and constant load segment data in terms of the Johnson-Cook model is facilitated by the approach proposed in [30]. Consistent with the Johnson-Cook analysis considered in [46], the approach is scaler and describes measured 'microscopic' quantities. The scaler analysis of the indentation hardness considers the time dependent plastic strain rate in terms and the time dependent indentation depth $h(t)$, through the expression:

$$
\sigma(t)=E\left[\epsilon_{o}-\frac{\lambda}{h(t)} \int_{0}^{t} h(\tau) \dot{\epsilon_{p}} d \tau\right]
$$


Where, $\epsilon_{o}$ is the Berkovich indentation characteristic strain and $\lambda$ is a constant determined by the boundary conditions that:

$$
\text { if } \int_{0}^{t} h(\tau)\left(\dot{\epsilon_{p}}\right) d \tau=h(t) \text {, then } \sigma(t)=0
$$

Thus, determining that $\lambda=\epsilon_{o}$. The characteristic strain, $\epsilon_{o}$, is a consequence of the Berkovich indentation geometric self-similarity, which refers to the fact that in a homogeneous material at a particular strain rate, the hardness is (ideally) not a function of indentation depth. Typically, the characteristic strain is considered to be $\epsilon_{o} \sim 0.1$. Following the analysis in [30], the plastic strain rate may be approximated as:

$$
\dot{\epsilon_{p}}=\frac{\dot{h}}{h}\left(1-\frac{\sigma(t)}{E \epsilon_{o}}\right)
$$

The hold segment data for the relationship between $\dot{h} / h$ and $\sigma(t)$ via curve fitting has the form consistent with the Johnson-Cook model as

$$
\frac{\dot{h}}{h}=\dot{\epsilon_{o}} \exp \left(\frac{\sigma(t)-\sigma_{o}}{C \sigma_{o}}\right)
$$

Where $\sigma_{o}$ is the athermal stress. Substitution of equation (4.4(b)) into equation (4.4(a)) allows for a direct comparison between the Johnson-Cook model and the 
Berkovich indentation constant load (hold) stress relaxation segment. An example is given in figure (4.3) where the fitting parameters relating equation (4.4(b)) to the experimental measurements from the indentation test.

Though, the Johnson Cook strain dependence model is often referred to as 'nonphysical' as there is no a priori physical meaning given to the fitting parameters such as $\sigma_{o}$, $\dot{\epsilon_{o}}, C$. However, it is noted below that there is a correlation between the Johnson-Cook approach and the "physical" approach originally presented by G. Schoeck and G. Gibbs in equation (4.5) [47-50].

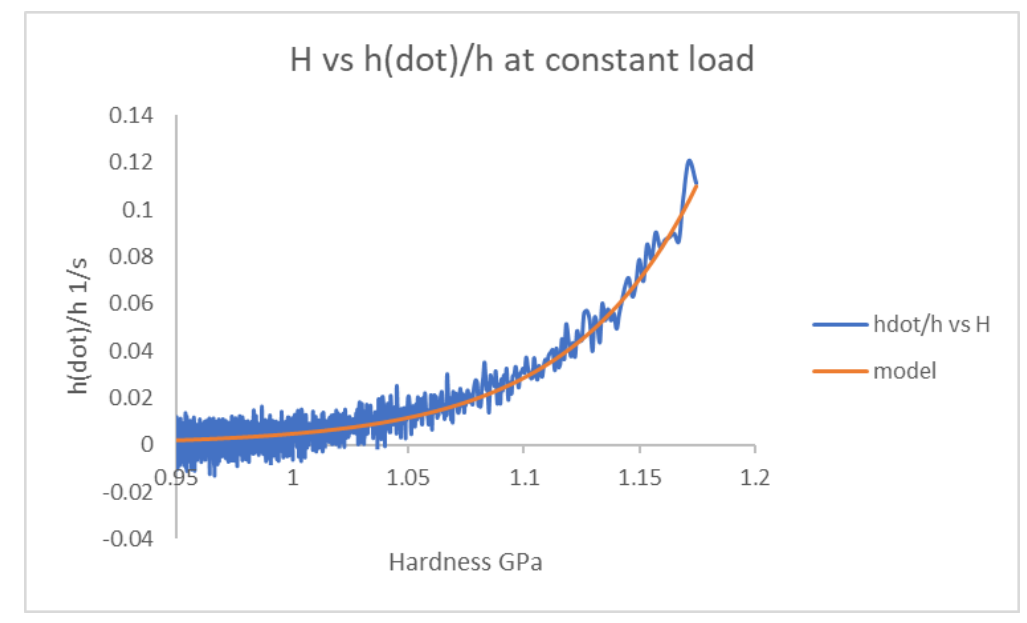

Figure 4.3. A representative curve for strain rate vs hardness with the experimental data and the Johnson-Cook model data.

$$
\dot{\varepsilon_{t}}=\dot{\varepsilon_{o}} * \exp \left[-\left[\frac{\Delta g-\left(\sigma-\sigma_{o}\right)}{K * T}\right]\right]
$$


Further, the relation between $\sigma_{o}$ and $C$ can be justified through the readings of A. G. Evans and R. D. Rawlings [51]. Evans note that the strain rate dependence on the effective stress is as given in the equation (4.6).

$$
\dot{\varepsilon}_{t}=\rho \cdot b \cdot x \cdot \vartheta_{o} \cdot \cos (\theta) \cdot \cos (\varnothing) \cdot \exp \left[-\left[\frac{\Delta g-V \cdot\left(\sigma-\sigma_{o}\right)}{K T}\right]\right]
$$

Where, $\mathrm{V}$ is the activation volume, $\rho$ is the mobile dislocation density, $\mathrm{b}$ is the Burgers vector, magnitude $\mathrm{x}$ is the slip length, and the trigonometric terms give the Schmid factor. To correlated equation (4.6) with the Johnson-Cook approach provided in the equation (4.5), if $\dot{\epsilon}_{o}$ is written in terms of mobile dislocation density ( $\rho$ ), Burger's vector (b), glide length $(\mathrm{x})$, activation energy $(\Delta \mathrm{g})$, as:

$$
\dot{\varepsilon_{o}}=\rho \cdot b \cdot x \cdot \vartheta_{o} \cdot \cos (\theta) \cdot \cos (\varnothing) \cdot \exp \left[-\left[\frac{\Delta g}{K T}\right]\right]
$$

Then,

$$
\dot{\varepsilon_{t}}=\dot{\varepsilon_{o}} \cdot \exp \left[\frac{V\left(\sigma-\sigma_{o}\right)}{K T}\right]=\dot{\varepsilon_{o}} \cdot \exp \left[\frac{\sigma}{\sigma_{o}}-1\right]
$$

From the equation (4.8), the Johnson-Cook parameter C can be expressed using the physical quantities, i.e. activation volume, $\mathrm{V}$ and athermal stress, $\sigma_{o}$ as: 


$$
C=\frac{K T}{V \cdot \sigma_{o}}
$$

Another widely used physical model presented by Kocks and Evans [51-52] is utilized to understand dislocation dynamics through interaction with the obstacles such as solute atoms. This model allows determination of critical stress $\left(\tau_{c}\right)$ i.e. stress required to overcome energy barriers. Two physical models, Gibbs-Schnoeck model and Kocks-Evans model originally present conflicting approached while addressing analytical theory of strain rate sensitivity.

\begin{tabular}{|c|c|c|}
\hline $\begin{array}{c}\text { Johnson-Cook } \\
\text { Model (fitting } \\
\text { parameters) }\end{array}$ & $\begin{array}{c}\text { Schoeck-Gibbs Model } \\
\text { Physical Interpretation }\end{array}$ & $\begin{array}{c}\text { Evans-Kocks Model } \\
\text { Physical Interpretation }\end{array}$ \\
\hline$\dot{\varepsilon}_{t}=\dot{\varepsilon}_{o} \exp \left[\frac{1}{C} *\left(\frac{\sigma}{\sigma_{o}}-1\right)\right]$ & $\dot{\varepsilon}_{t}=\rho b x \vartheta_{o} \exp \left[-\left(\frac{\Delta g-V\left(\sigma-\sigma_{o}\right)}{K T}\right)\right]$ & $\dot{\varepsilon}_{t}=\dot{\varepsilon}_{o} * \exp \left[-\frac{\boldsymbol{F}}{\boldsymbol{K} \boldsymbol{T}} *\left(\mathbf{1}-\frac{\boldsymbol{\sigma}-\boldsymbol{\sigma}_{o}}{\boldsymbol{\tau}_{\boldsymbol{c}}}\right)\right]$ \\
\hline$\dot{\varepsilon}_{o}$ & $\rho b x \vartheta_{o} \exp \left[-\left(\frac{\Delta g}{K T}\right)\right]$ & $\dot{\varepsilon}_{o}$ \\
\hline$C$ & $\frac{K T}{V \sigma_{o}}$ & $\frac{K T}{F}$ \\
\hline$\sigma_{o}$ & $\sigma_{o}$ & $\sigma_{o}$ \\
\hline
\end{tabular}

Table 4.3. The Johnson-Cook parameters and their physical interpretation using SchoeckGibbs Model [47-50] and Evans-Kocks Model [51-52].

The difference in the exponential argument for the thermally activated dislocation behavior between Schoeck-Gibbs and Evans-Kocks is related to the reference stress 
relationship to the applied stress. Schoeck-Gibbs uses a back-stress term, $\sigma_{o}$, to limit the effect of the applied stress. This is the same stress that appears in the Johnson-Cook model at minimum strain rate, $\dot{\varepsilon_{p}}=\dot{\varepsilon_{o}}$. Alternatively, Evans-Kocks uses a reference stress corresponding to the critical stress, $\tau_{c}$ where no thermal activation is required to bypass an obstacle of strength, F (Joules). Thus, Schoeck-Gibbs focus on a lower limit of stress to drive the strain rate while Evans-Kocks examine an upper limit of the stress that can be obtained by dislocations pushing through a certain type of obstacle. However, authors propose that the critical stress associated with Evans-Kocks (EK) can be defined through the Schoeck-Gibbs (SG) model, allowing an analysis to show that the EK and SG approaches are equivalent while providing a chance that the critical stress can be defined using experimentally measurable quantities.

The analysis starts by noting that the argument of the exponential term in the Schoeck-Gibbs model for strain rate is:

$$
-\frac{\Delta g}{K T}+\frac{V}{K T} \cdot\left(\sigma-\sigma_{o}\right)
$$

And the argument of the exponential (in the simplest form) for the exponential in the Evans-Kocks theory has the form [51-53]

$$
-\frac{F}{K T} \cdot\left[1-\frac{\sigma-\sigma_{o}}{\tau_{c}}\right]
$$

Where $\tau_{c}$ is the critical stress where the energy barrier to plastic deformation 'disappears', i.e. the dislocation obstacle energy is overcome by the applied stress. 
There are certain parameter definitions which allows the convergence of the Schoeck-Gibbs and Evans-Kocks theories. The convergence requires the condition that when $\sigma-\sigma_{o}=0$ then the SG and $\mathrm{EK}$ are equivalent, or that $\mathrm{F}=\Delta g$. In addition, the condition that the stress is high enough to overcome the activation barrier without thermal activation must also be equivalent.

For Schoeck-Gibbs model, this critical stress is the solution to

$$
-\Delta g+V\left(\sigma-\sigma_{o}\right)=0
$$

Or

$$
\sigma=\frac{\Delta g+V\left(\sigma_{o}\right)}{V}
$$

For Evans-Kocks model,

$$
-\frac{\Delta g}{K T} \cdot\left[1-\frac{\sigma-\sigma_{o}}{\tau_{c}}\right]=0
$$

Or

$$
\sigma=\tau_{c}+\sigma_{o}
$$

Setting $\sigma$ from both models equal:

$$
\tau_{c}=\frac{\Delta g}{V}
$$


We note the importance of this result as the activation volume concept may now be incorporated within the EK theory through an equivalence requirement with the SG approach. To summarize, the equivalence of SG and EK determines that the obstacle strength in the EK approach is the same as the activation energy in the SG analysis, and the critical shear stress in the EK approach is the activation energy divided by the activation volume, $\Delta g / \mathrm{V}$.

As illustrated here, the Johnson-Cook model in conjunction with physical models presented by Gibbs \& Schoeck and Kocks \& Evans are utilized in this work to investigate local grain boundary mechanical behavior through stress-strain rate responses mainly under constant load conditions, but also augmented by analysis of constant loading rate variations.

The Johnson-Cook-EK-SG analysis is also applied (for the first time) to the analysis of grain boundary pop-in events in pure and carbon doped iron. The modification the Johnson-Cook model facilitates estimation of athermal stress as a function of depth during pop-in event. Unlike stress relaxation test conducted at the constant load of $24 \mathrm{mN}$ for 60 sec duration, the grain boundary events are relatively rapid variations in strain rate, occurring within a second or less. The requirement of constant load over stress-strain rate response evaluation is fulfilled as the pop-in occurs within such a short period of time that the load only changes by less than 1-2\%, essentially giving a constant load during the event. The following analysis presents the approach used for the athermal stress measurements on the onset of displacement burst or pop-in:

1. Modified Johnson-Cook model to determine athermal stress as a function of depth:

The original Johnson-Cook model is implemented to study stress relaxation response under a constant applied load. The grain boundary pop-in event represents a near instantaneous drop in hardness, proposed to be a result of grain boundary yielding. As popin occurs at a particular critical load, the Johnson-Cook model is utilized to analyze 
hardness - strain rate response during a very short period at the onset of pop-in. The analysis for athermal stress as a function of depth is presented below:

It is important to note that this analysis utilizes certain set of assumptions and mainly serves as an illustration of difference in the athermal stress variation during grain boundary pop-in, for indentations at the grain boundary in pure iron and carbon doped iron.

For the indentation that exhibits grain boundary pop-in during loading and to be assessed using modified Johnson-Cook model, firstly the stress relaxation hardness - strain rate response is analyzed using original Johnson-Cook approach. This analysis facilitates determination of activation volume $(\mathrm{V})$ and characteristic strain rate $\left(\dot{\varepsilon}_{o}\right)$.

The modified Johnson-Cook analysis assumes the activation volume (V) and characteristic strain rate $\left(\dot{\varepsilon}_{o}\right)$ parameters from the stress relaxation during constant load hold segment to be the relevant values to be used for modelling the pop-in event. This assumption can be rationalized by the observation that the athermal stress values determined in the constant load experiments drop significantly for the grain boundaries that have 'popped' as compared to the grain boundaries that have not popped. This assumption allows the determination of the pop-in event as a rapid reduction in athermal stress. By rearranging original Johnson-Cook model as shown below, where hardness is taken to be a function of depth:

$$
\sigma_{o}(h)=\sigma(h)-\left(\frac{K T}{V} * \ln \left(\frac{\dot{\varepsilon}_{h}}{\dot{\varepsilon}_{o}}\right)\right)
$$

This approach facilitates measurement of athermal stress as a function of depth, during pop-in event, but it is a limiting case analysis as the only parameter considered to change during the pop-in events is the athermal stress. 


\subsection{MATERIALS AND METHODS:}

A high-purity (99.999\%) iron sample (purchased from Goodfellow USA) 10mm diameter rod in as drawn condition was cut into a thin disk of $4 \mathrm{~mm}$ thickness. This disk further underwent a carefully devised sample preparation process which includes steps such as metallographic sample preparation, $\mathrm{H}_{2}$ :Ar based grain growth heat treatment $(>40$ hours at $\left.800^{\circ} \mathrm{C}\right)$, ion-milling of polished surface, and annealing heat treatment $\left(850^{\circ} \mathrm{C}\right)$. More details about the sample preparation process are provided in the [30].
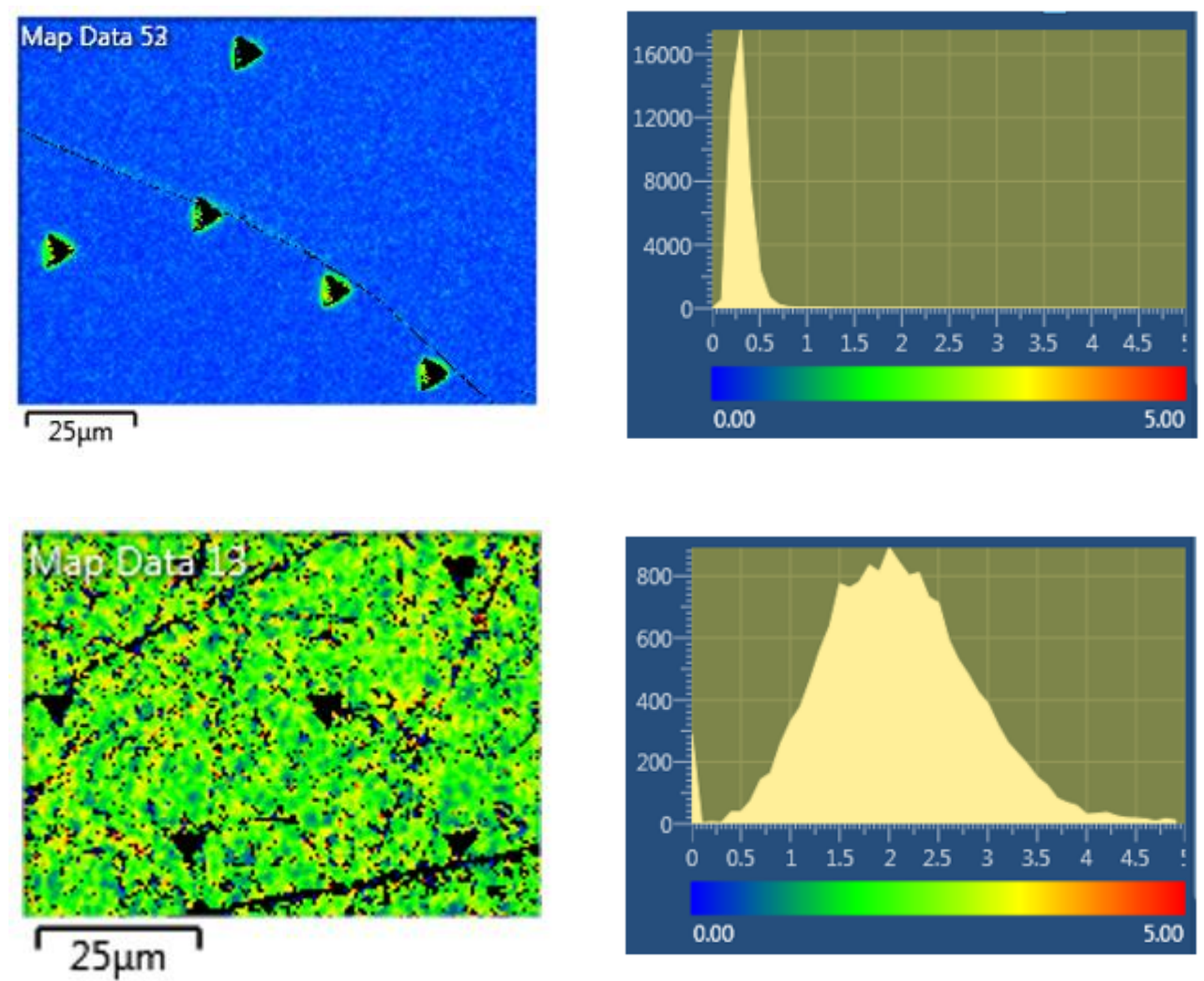

Figure 4.4. The EBSD based KAM and PQ data for annealed and deformed surface of iron [30]

The Kernel Average Misorientation (KAM) maps and Picture Quality (PQ) analysis were obtained using an Oxford electron back scatter diffraction system interfaced with the 
FEI XL40 environmental scanning electron microscope, to ensure the sample is free of sub-surface defects, as shown in figure (4.4). Additionally, the specimen was tested using nanoindentation to examine shallow depth pop-in behavior which represents dislocation nucleation in the low defect-density surface (refer figure (4.5)).

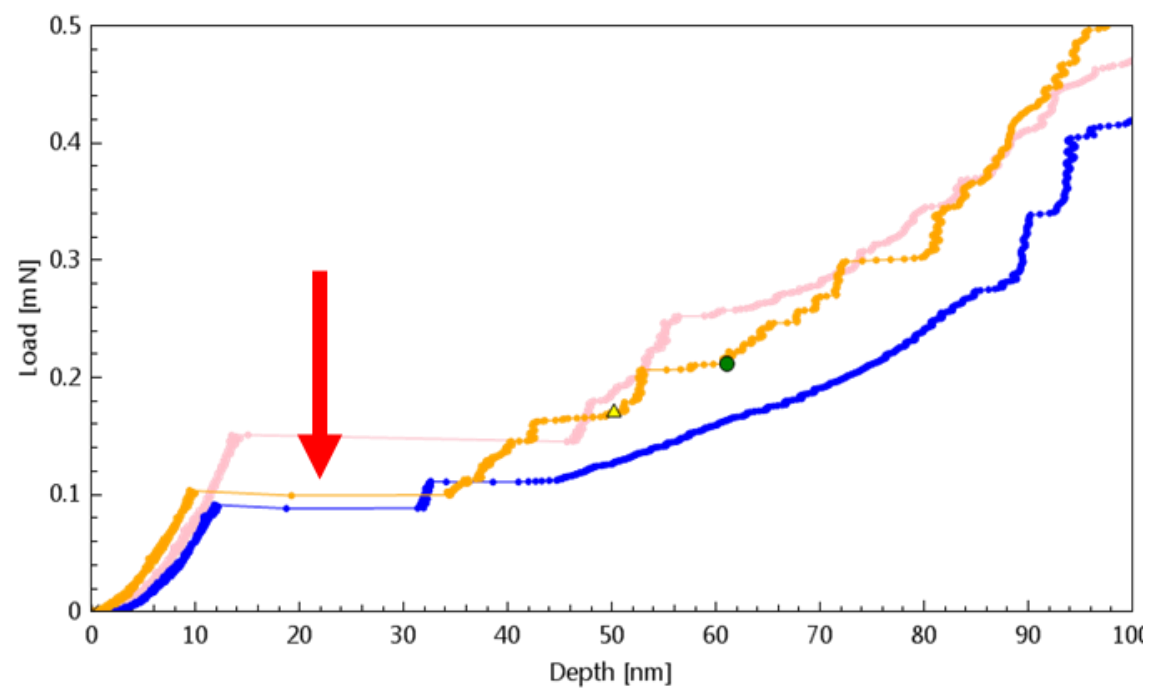

Figure 4.5. A representative load-depth plot with shallow depth pop-in, as a result of dislocation nucleation [30].

The free surface and the grain boundary were examined in the pure iron as well as carbon doped condition, to examine influence of chemistry on grain boundary indentation results. The carbon doping method used during this work differs from the method presented in the chapter 3 , and is outlined below:

The carbon doping was carried out by vapor deposition of carbon onto the $\mathrm{Fe}$ sample surface, followed by a drive-in heat treating in vacuum furnace. The vapor deposition was carried out using the Cressington 208 HR high resolution sputter coater. 
The amount of carbon deposited was set to be about $15 \mathrm{~nm}$ thick. A polished brass standard was used to approximate the carbon film thickness, using the method presented by [54]. The interference color produced on the brass surface upon carbon deposition during sputtering process is used to approximately determine the carbon layer thickness, as shown in the figure (4.6).

After carbon deposition is performed on a pure iron sample, followed by a drive-in diffusion heat treatment at $\sim 650^{\circ} \mathrm{C}$ for 3 hours, mathematical analysis is performed to study carbon concentration profile beneath the surface. The Gibbs Adsorption Isotherm predicts preferential segregation of solute atoms near grain boundary region compared to the grain interior, by energy minimization principle. A grain boundary and volume diffusion study performed in Fe-C system by [55] is utilized for understanding carbon distribution profile upon drive-in diffusion process.

\section{.. Interference Colors of Carbon Films on Polished Brass Substrates*}

\begin{tabular}{cl}
\hline Approximate Thickness & \multicolumn{1}{c}{ Color } \\
\hline $150 \AA$ & Orange \\
200 & Indigo Red \\
250 & Blue
\end{tabular}

Figure 4.6. Interference color of carbon film and corresponding film thickness when carbon deposition is performed on the standard brass substrate [54]. 

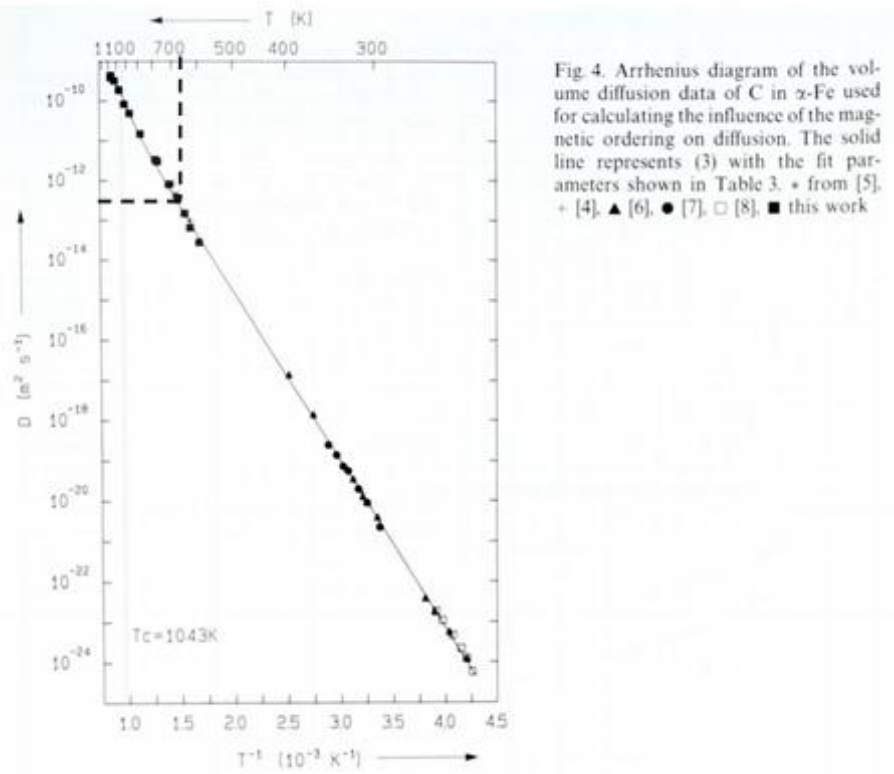

Fig 4 A Arhenius diagram of the vol. tea of $C$ in $\alpha$-Fe used

Figure 4.7. A plot of volume diffusion coefficient vs temperature for $\mathrm{C}$ in $\alpha$-iron [55]

Using Arrhenius diagram for carbon volume diffusion in iron [plotted based on various experimental studies], $D_{v}=5 * 10^{-13}\left(\mathrm{~m}^{2} \mathrm{~s}^{-1}\right)$. This study utilized Whipple solution to estimate grain boundary diffusion product, $\mathrm{P}$ given as:

$$
P=s \delta D_{g b}=1.322 * \sqrt{\frac{D_{v}}{t}}\left[-\frac{\partial \ln \bar{c}}{\partial z^{6 / 5}}\right]^{-5 / 3}
$$

Where $s$ - segregation factor $\left(s=\frac{c_{g b}}{c_{v}}\right)[56], \delta$ - grain boundary thickness $=0.5 \mathrm{~nm}, D_{g b^{-}}$ grain boundary diffusion coefficient, $D_{v}$ - volume diffusion coefficient, $\mathrm{t}$ - time, $z-$ depth, $\bar{c}-$ mean tracer concentration at a depth $z$. 
The grain boundary diffusion product $\mathrm{P}$ is plotted against temperature $\mathrm{T}$ using experimental data as shown below. From the figure, $\mathrm{P}$ corresponding to the carbon grain boundary diffusion in $\alpha$-iron at $\sim 650^{\circ} \mathrm{C}=\sim 4 * 10^{-16}\left(\mathrm{~m}^{3} \mathrm{~s}^{-1}\right)$

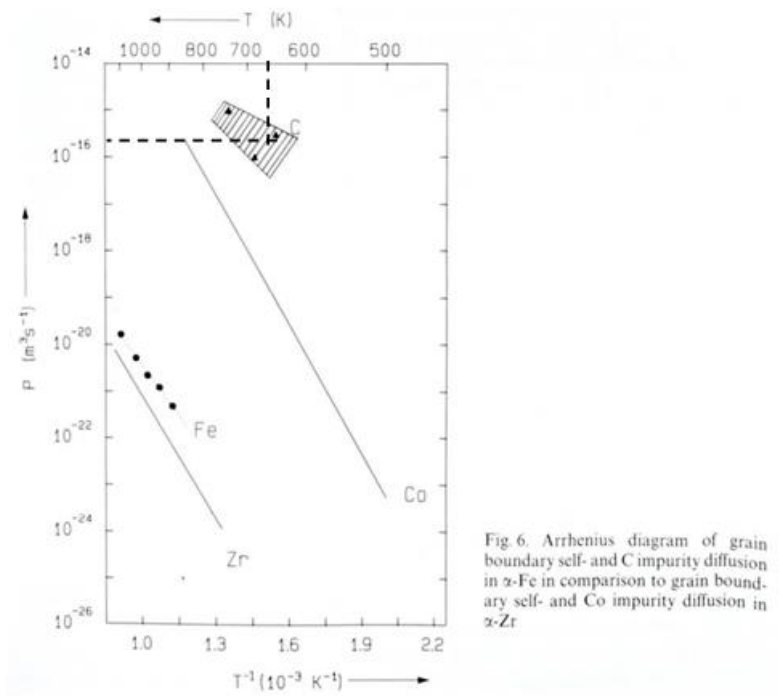

Figure 4.8. The Arrhenius diagram of grain boundary self and carbon impurity diffusion in $\alpha$-iron [55]

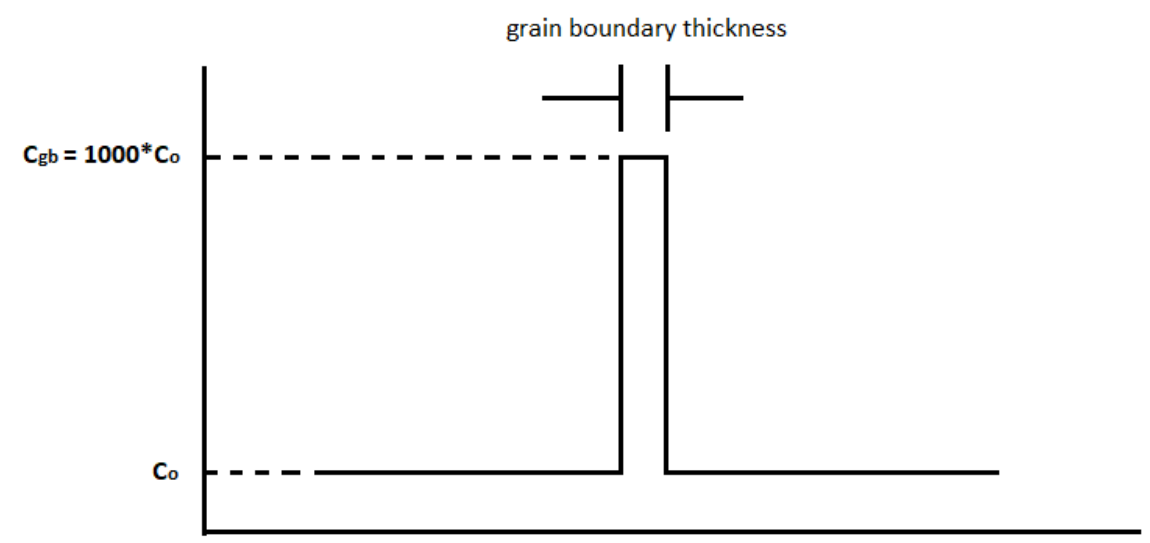

Figure 4.9. Schematic of carbon concentration profile in Fe-C system in equilibrium, indicating significant segregation at the grain boundary compared to adjacent grain interior, as predicted by the Gibbs Adsorption Isotherm. 
According to E. Budke's study s value for carbon grain boundary diffusion is considerably lower than $\left(10^{5}-10^{6}\right)$, so considering $\mathrm{s}=10^{3}, D_{g b}=8 * 10^{-10}\left(\mathrm{~m}^{2} \mathrm{~s}^{-1}\right)$ As segregation factor $(\mathrm{s}) \sim 10^{3}$, at equilibrium without diffusion in the $\mathrm{Fe}-\mathrm{C}$ system across volume and grain boundary carbon concentration profile would look as shown in the schematic in figure (4.9), indicating significant grain boundary segregation:

Further using the Whipple-Fisher grain boundary diffusion analysis [57-58], to examine the carbon concentration profile across the grain boundary, as shown below:

$$
\eta=\frac{Y}{\sqrt{D_{v^{* t}}}}
$$

$$
\beta=\frac{D_{G B}}{D_{v}} * \frac{\frac{1}{2} * \delta}{\sqrt{D_{v^{* t}}}}
$$

Where, $\eta \& \beta$ are dimensionless parameters, $Y$ - diffusion length, $D_{v} \& D_{G B}$ - volume diffusion coefficient and grain boundary diffusion coefficient for carbon, respectively, $\delta$ grain boundary and carbon deposition layer thickness, $t$ - drive-in heat treatment duration.

Using dimensionless parameters determined earlier, Fisher contour angle can be estimated as:

$$
\theta=\frac{360}{2 \pi} * \operatorname{acot}\left(\pi^{-\frac{1}{4}} * \beta^{-\frac{1}{4}}\right)
$$


Using $D_{v}=5 * 10^{-13} \frac{\mathrm{m}}{\mathrm{s}^{2}}, D_{G B}=8 * 10^{-10} \frac{\mathrm{m}}{\mathrm{s}^{2}}, Y=10 \mu \mathrm{m}, t=10^{4} \mathrm{sec}, \delta=0.5 \mathrm{~nm}$,

Then, Fisher contour angle $=86.81^{\circ}$

Corresponding carbon concentration profile is shown below:

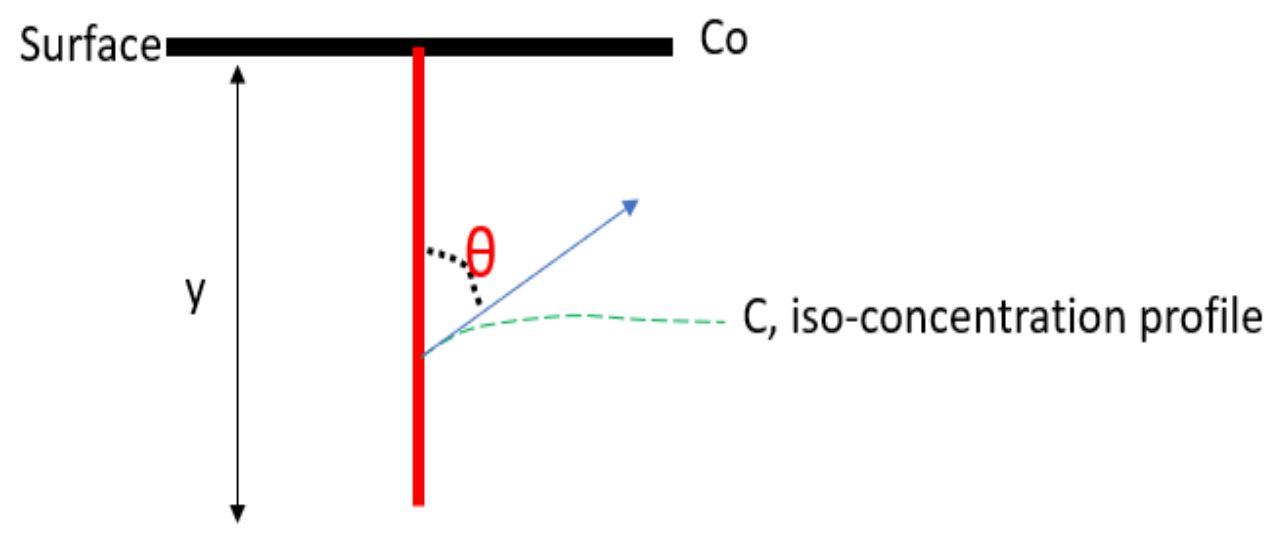

Figure 4.10. A schematic of carbon concentration gradient perpendicular to the grain boundary into the grain interior, predicted based on Whipple-Fisher contour angle measurement. [57-58]

It is emphasized that with the value of the Fisher contour angle approximately 90 degrees at $10 \mu \mathrm{m}$ depth implies that the carbon concentration profile approaches that shown in Figure (4.9) over the depths that would influence the indentation hardness. The concentration throughout the diffusion zone from subsequent 'drive in' diffusion is approximated as $\sim 1^{*} 10^{-3} \mathrm{wt} \%$. This analysis indicates a homogeneous distribution of carbon over the entire free surface of the Fe sample out to a depth of $10 \times 10^{-6} \mathrm{~m}$.

Further sample characterization was performed using a focused beam of highenergy (30 kV) gallium ions, the Hitachi FB-2000A (Krefeld, Germany) focused ion beam (FIB) mill and the FEI (Einhoven, Netherlands) $200 \mathrm{kV}$ Titan Themis scanning transmission electron microscope (S-TEM), to confirm the grain boundary perpendicular 
to the free surface. The diffraction pattern confirmed that no additional phases such as $\mathrm{Fe}_{3} \mathrm{C}$ were detected. Details of these stages are provided in chapter 3[30].

Nanoindentation experiments were performed using a diamond Berkovich indenter tip and the iNano (Nanomechanics Inc.-KLA Tencor, Oak Ridge, Tennessee). As presented in the load-time history, nanoindentation experiment begins with the constant

loading rate, $\left(\frac{\dot{P}}{P}\right)$, followed by $60 \mathrm{sec}$ long constant load hold segment at $P_{\max }=24 \mathrm{mN}$. This constant load hold segment represents stress relaxation test. Then the continuous stiffness measurement (CSM) protocol is utilized for $120 \mathrm{sec}$ duration followed by a rapid unloading $(<1 \mathrm{sec})$ and $80 \mathrm{sec}$ of thermal drift measurements to determine thermal contribution to the depth measurements. The strain rate study in the grain interior involved utilization of seven different loading rates (i.e. 0.05/s, 0.1/s, 0.2/s, 1/s, 2/s, 4/s, 8/s). Similarly, grain boundary indentations were carried out at $0.2 / \mathrm{s}$ and $4 / \mathrm{s}$ of loading rate.

\subsection{RESULT AND DISCUSSION:}

The result and discussion section is organized to parallel Section 4.2.2. The analysis of hardness relaxation experiments (constant load) augmented with analysis of hardness: strain rate behavior at different constant loading rates is presented first. The comparison of the grain interior indentation behavior with the remote grain boundary indentation behavior is emphasized. The analysis of the grain boundary pop-in events is then analyzed as described in 4.2.2

\subsubsection{The Analysis of the Indentation Hardness Relaxation Behavior:}

\subsubsection{Grain interior indentations:}

The nanoindentation experiments are performed in the well-annealed pure iron grain interior and carbon doped grain interior, for both grain bounding the grain boundaries of interest, using the load-time history provided in the figure (4.2). Prior to the imposition 
of a constant load, these indentations are carried out at different strain rate or loading rates $\left(\frac{\dot{P}}{P}\right)$ [i.e., $\left.0.05,0.1,0.2,1,2,4,8(1 / \mathrm{s})\right]$ where 20 tests in a matrix of $4 \times 5$ are conducted at each strain rate in each grain. The use of multiple strain rates facilitates examination of strain rate effects on the dislocation dynamics and underlying plastic deformation mechanism and effects on the hardness relaxation during constant load hold segment of the indentation experiment. The stress-strain rate responses during the hardness relaxations are analyzed via the Johnson-Cook-EK-SG model-based approach outlined in the section 4.2.2 to facilitate the determination of physical parameters such as activation volume $V$, athermal stress $\left(\sigma_{o}\right)$, characteristic strain rates $\left(\dot{\varepsilon}_{o}\right)$. These physical entities measured from the grain interior tests in pure and carbon doped conditions are presented as a function of indentation strain rate $\left(\frac{\dot{P}}{P}\right)$ in figures (4.12-4.15). Figure (4.11) shows that the activation volume for the pure iron and carbon doped iron grain interiors decreases with increasing strain rate, while figure 4.12 shows that the effective stress $\left(\sigma-\sigma_{o}\right)$ increases with strain rate even though the athermal stress remains essentially constant. Finally, the characteristic strain rate, $\dot{\varepsilon}_{o}$, is seen to increase with increasing strain rate (stress).

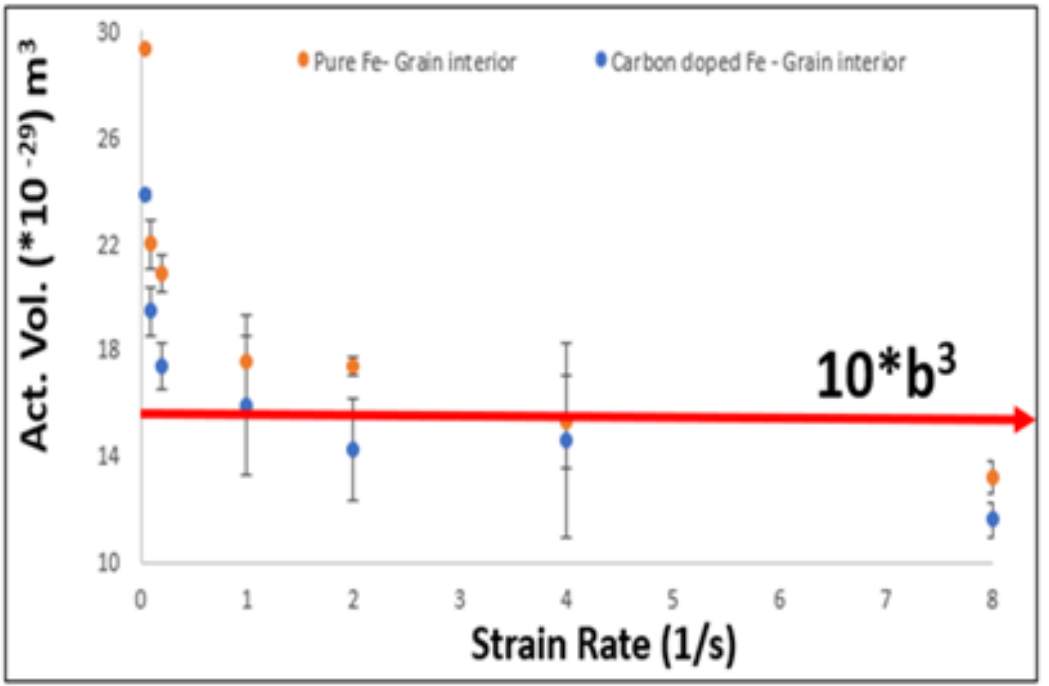

Figure 4.11. Activation volume variation as function of indentation strain rate. This result is very similar to bulk iron strain rate test analysis performed by F. A. Smidt [36] 
The activation volume measured through stress relaxation tests in the grain interior represents an area or volume of material influenced by the applied stress within which dislocations sweep through from the equilibrium condition. In general, the activation volume is a strong function of microstructural complexity wherein increased dislocation density due to higher applied strain rate (in effect stress) leads to decrease in activation volume. In the figure (4.11), the observed inverse relationship behavior between the stress (strain rate) and the activation volume can be understood in the context of dislocation bowing out event. A very simple model that sacrifices some accuracy for clarity examines a dislocation is bowing out between two pinning dislocation obstacles with arc length $(\mathrm{R})$ and breaks free from these obstacles, creating jogs of length $b$. In this case, activation volume $(V)$ is given as:

$$
V=R * b^{2}
$$

Where, $\mathrm{b}$ - Burger's vector magnitude, $\mathrm{R}$ - Dislocation arc length.

The stress required to cause the dislocation bow out can be crudely estimated as:

$$
\sigma=\beta \frac{G * b}{R}
$$

Where $\beta$ is a parameter relating to the geometry of the dislocation jog formation. So, the activation volume for dislocation de-pinning is given by,

$$
V=\frac{\beta * G * b^{3}}{\sigma}
$$


reproducing the general behavior observed figure (4.11), (4.12) and (4.14). The behavior in figure 4.13 where the characteristic strain rate is seen to increase with strain rate (stress). The simplest rationalization for this observation is developed from equation 4.7, where the characteristic strain rate increases linearly with the mobile dislocation density. Argon [37] has tied the mobile dislocation density to the applied stress through a simple function as:

$$
\rho_{\text {mobile }}=\alpha *\left(\frac{\sigma}{G b}\right)^{2}
$$

Where, $\alpha=1$, and this function explains as indentation strain rate increases, the amount of mobile dislocations introduced within deformation region increases, resulting in closely packed dislocations.

A more complicated, but perhaps more illuminating analysis relating applied stress to mobile dislocations has been presented by Neri and Nix [59]. This analysis is considered in Appendix B4. The argument does predict an increase in mobile dislocation density with increasing stress at constant athermal stress as plotted in figure (4.13 b), although the predicted $30 \%$ increase in mobile dislocation density with the observed stress increase does not explain the large increase in characteristic strain rate observed in figure $(4.13 \mathrm{a})$.

With respect to the grain interior activation volume results presented in this study, it is worth noting that the high purity iron stress relaxation studies conducted using tensile tests by F. A. Smidt [36] concluded that the activation volume is strongly associated with the strain rate, where the activation volume appears to be a strong function of strain rate at smaller strain rates but as strain rate increases, activation volume reaches plateau with values about $10 \mathrm{~b}^{3}$. This result by Smidt is similar to the results shown in figure (4.11). The work performed by Taylor [60] and J. W. Christian and B. C. Masters on thermally activated deformations in BCC metals [61-62] reiterates similar observation. These studies 
suggest that such observation is a characteristic of Peierls mechanism dominating at very high stresses.

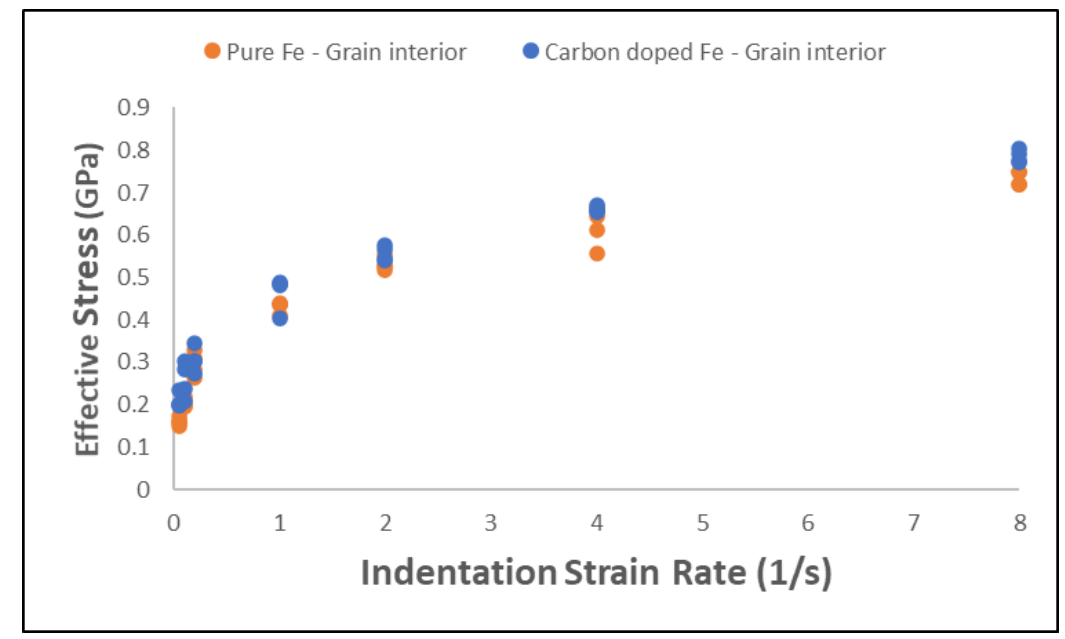

Figure 4.12. Effective stress $\left(\sigma-\sigma_{o}\right)$ variation as a function of indentation strain rate

The Berkovich indenter exhibits geometric self-similarity, strain imposed in the vicinity of the indenter is constant (averaged $\sim 8 \%$ ). The athermal stress (or back-stress) is a function of total dislocation density (including mobile dislocation, immobile dislocations, geometrically necessary dislocations). As the plastic strain by the Berkovich nano-indenter is more or less the constant, the amount of total dislocations introduced in the material and in turn athermal stress appears to remain constant, as shown in the figure (4.14).

The grain interior indentation results in figure (4.11) to (4.14) were also examined for chemistry effects (solute strengthening), although the analysis in equations (4.18) to (4.21) suggest a very low carbon concentration in the grain interior. The activation volume measured in figure (4.11) at any indentation strain rate in pure and carbon doped grain interior is statistically indistinguishable. This observation suggests that the mechanism of plastic 


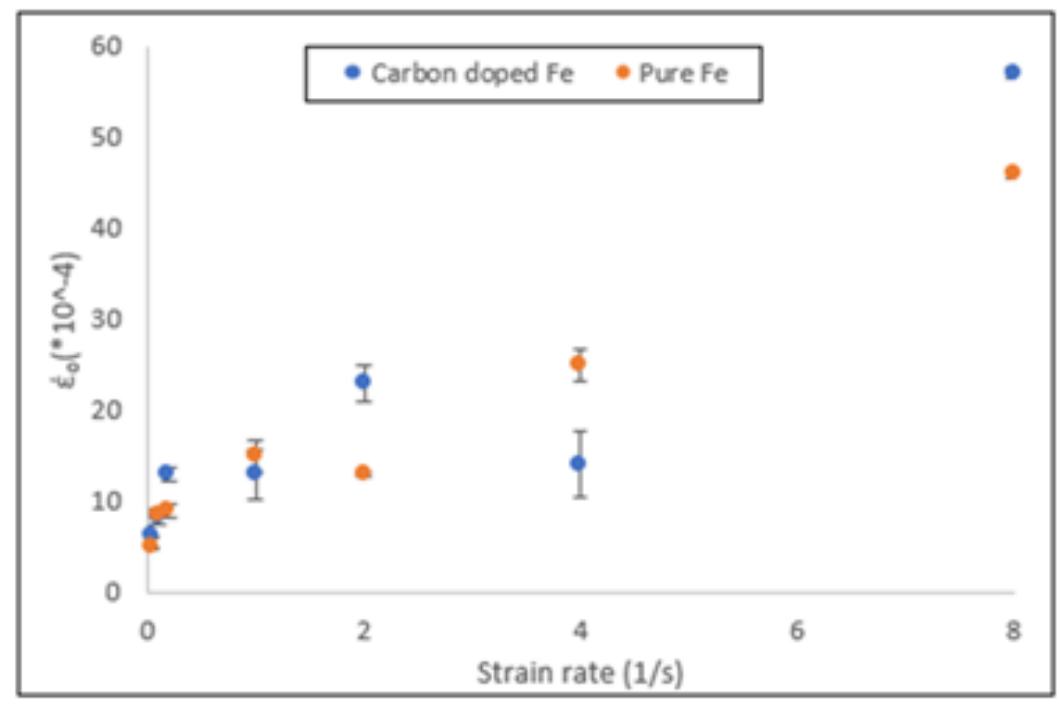

(a)

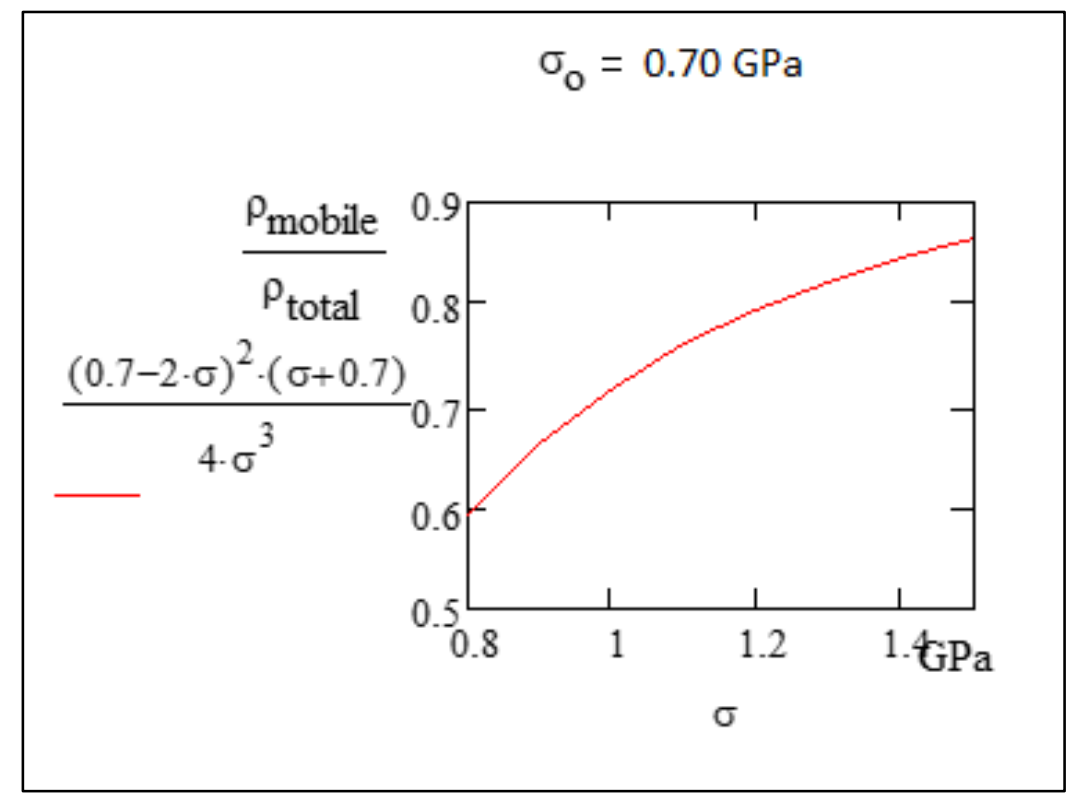

(b)

Figure 4.13. (a) The characteristic strain rate variation as a function of indentation strain rate, for the grain interior in pure iron and carbon doped iron. (b) The predicted increase in mobile dislocation density by Neri et al [59]. 


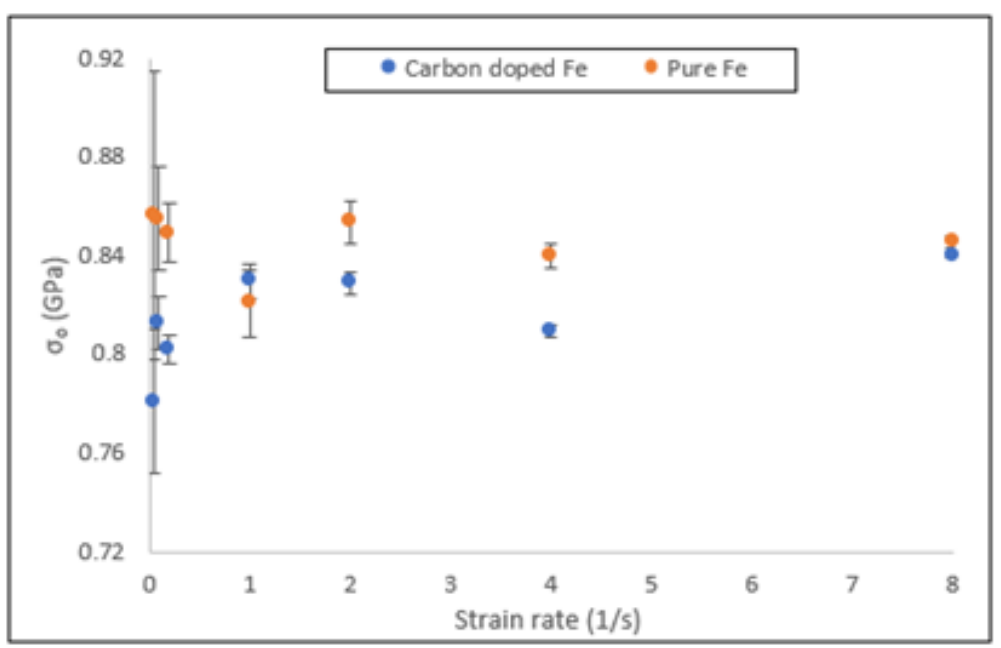

Figure 4.14. The athermal stress variation as a function of indentation strain rate, for the grain interior in pure iron and carbon doped iron.

deformation is unaffected by the solute doping in the grain interior of iron. The activation volume is considered to represent deformation mechanism governing plastic deformation [63]. According to the solid solution strengthening mechanism, the solute doping in the grain interior may have an effect on the characteristic strain rate and athermal stresses, as solute atoms may act as an additional obstacle source for the dislocation motion. However, the results presented in the figure (4.13 (a)) does not appear to show any consistent trend in the chemistry effects. This result is interpreted based on the calculations of the low carbon concentrations expected in the grain interior from the doping process (pg. 129). Finally, the athermal stress in figure (4.14) does show a statistically significant chemistry effect at $0.2 / \mathrm{s}$ strain rates, wherein carbon doped athermal stress values are higher than that observed in the pure iron. This may be attributed to the solid solution strengthening effects causing an increase in the stress required to cut (and thus displace) an immobile forest dislocation, perhaps due to carbon segregation to the immobile dislocation. 


\subsubsection{Grain boundary indentations:}

Following the analysis of strain rate effects in the grain interior with and without carbon, the approach is extended to analyze grain boundary indentations in pure and carbon doped conditions. In this work, 4 general grain boundaries with varying tilt-twist nature (details in table 4.4) are examined in pure and carbon doped condition, to understand a contribution of grain boundary chemistry in the strengthening mechanism. It is important to note that the grain boundary results discussed here utilize remote indentation geometry. The indentation experiments are conducted using the same load-time history presented in the figure (4.2) at the loading rate of $0.2 / \mathrm{s}$ and $4 / \mathrm{s}$. Unfortunately, the requirement to carry out multiple indentations with an indentation spacing of $30 \mu \mathrm{m}$ on the same grain boundary to determine statistical significance limits the design of experiment to only these two strain rates.

\begin{tabular}{|c|c|c|c|c|c|c|c|c|}
\hline \multirow{2}{*}{$\begin{array}{c}\text { Grain } \\
\text { Boundary }\end{array}$} & \multicolumn{2}{|c|}{ Pattern Center } & \multicolumn{2}{|c|}{ Grain Normal } & \multicolumn{2}{|c|}{ GB planes } & \multicolumn{2}{|c|}{ Misorientation } \\
\hline & G-1 & G-2 & G-1 & G-2 & G-1 & G-2 & axis & angle \\
\hline GB1 & {$\left[\begin{array}{lll}4 & 7 & 3\end{array}\right]$} & {$\left[\begin{array}{lll}3 & 4 & 8\end{array}\right]$} & {$\left[\begin{array}{lll}1 & 2 & 3\end{array}\right]$} & {$\left[\begin{array}{lll}0 & 0 & 1\end{array}\right]$} & $(\overline{3} 2 \overline{1})$ & $\left(\begin{array}{lll}\overline{2} & 1 & 0\end{array}\right)$ & {$\left[\begin{array}{lll}1 & 4 & 1\end{array}\right]$} & 41.80 \\
\hline GB2 & {$\left[\begin{array}{l}7 \overline{5} \\
7]\end{array}\right.$} & {$\left[\begin{array}{lll}5 & \overline{2} & 4\end{array}\right]$} & {$\left[\begin{array}{lll}1 & 0 & 1\end{array}\right]$} & {$\left[\begin{array}{lll}4 & 1 & 6\end{array}\right]$} & $\left(\begin{array}{lll}1 & 0 & \overline{1}\end{array}\right)$ & $\left(\begin{array}{lll}1 & 1 & \overline{1}\end{array}\right)$ & {$[2 \overline{1} 4]$} & 22.33 \\
\hline GB3 & {$\left[\begin{array}{lll}\overline{4} & 3 & 8\end{array}\right]$} & {$\left[\begin{array}{lll}\overline{1} & 2 & 8\end{array}\right]$} & {$\left[\begin{array}{lll}0 & 0 & 1\end{array}\right]$} & {$\left[\begin{array}{lll}1 & 1 & 4\end{array}\right]$} & $(\overline{1} \overline{2} 0)$ & $(\overline{5} \overline{2} 0)$ & {$\left[\begin{array}{lll}\overline{1} & 2 & \overline{4}\end{array}\right]$} & 37.37 \\
\hline GB4 & {$\left[\begin{array}{lll}\overline{2} & 3 & 7\end{array}\right]$} & {$\left[\begin{array}{lll}\overline{2} & 3 & 5\end{array}\right]$} & {$\left[\begin{array}{lll}2 & 1 & 8\end{array}\right]$} & {$\left[\begin{array}{lll}1 & 2 & 4\end{array}\right]$} & $(\overline{1} \overline{3} 1)$ & $(0 \overline{2} 1)$ & {$\left[\begin{array}{lll}\overline{4} & 0 & 3\end{array}\right]$} & 17.51 \\
\hline
\end{tabular}

Table 4.4. Grain boundary and adjacent grain interior orientation details from the EBSD analysis. 


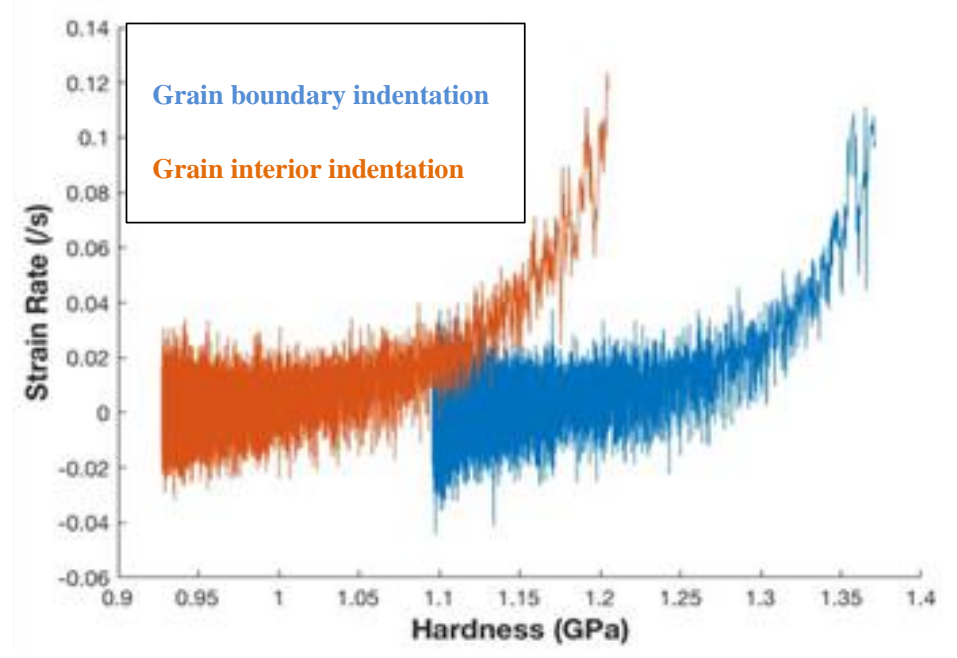

Figure 4.15. A representative strain rate - hardness profile for the grain boundary and grain interior.

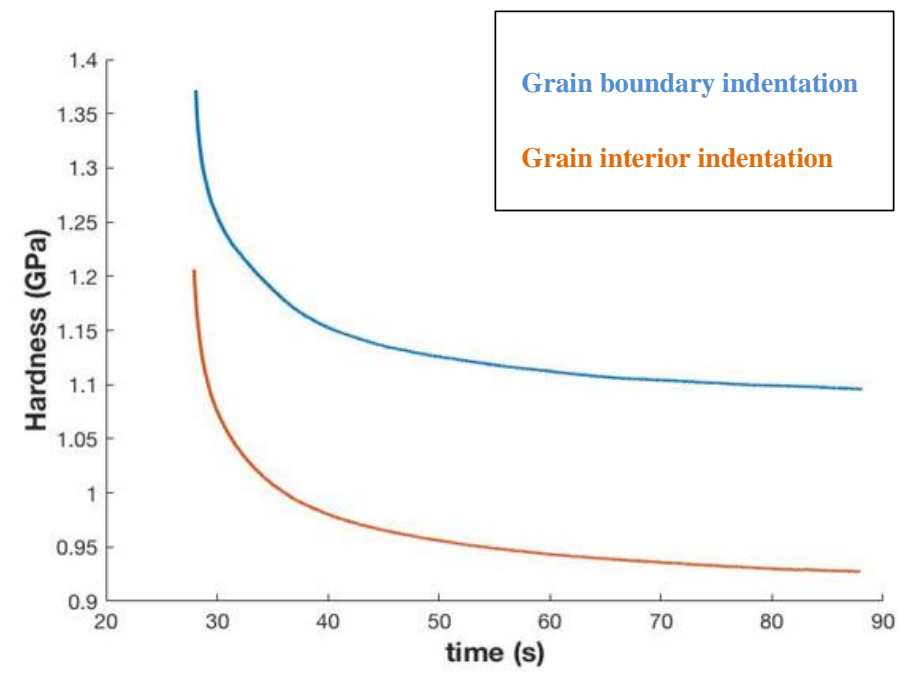

Figure 4.16. A representative hardness - depth profile for the grain boundary and grain interior indentation 
Figure (4.15) and (4.16) presents comparison of strain rate - stress and hardness time profile for grain boundary and grain interior, respectively, to emphasis that the strain rate - hardness response is unique to the microstructural (in) homogeneity presented by the grain boundary and grain interior.

The Johnson-Cook analysis is utilized to estimate fitting parameters associated with the grain boundary hardness relaxation curves initiated during a constant load experiment from each of the two strain rates, in pure and carbon doped conditions. The physical properties such as athermal stress, activation volume and characteristic strain rates are
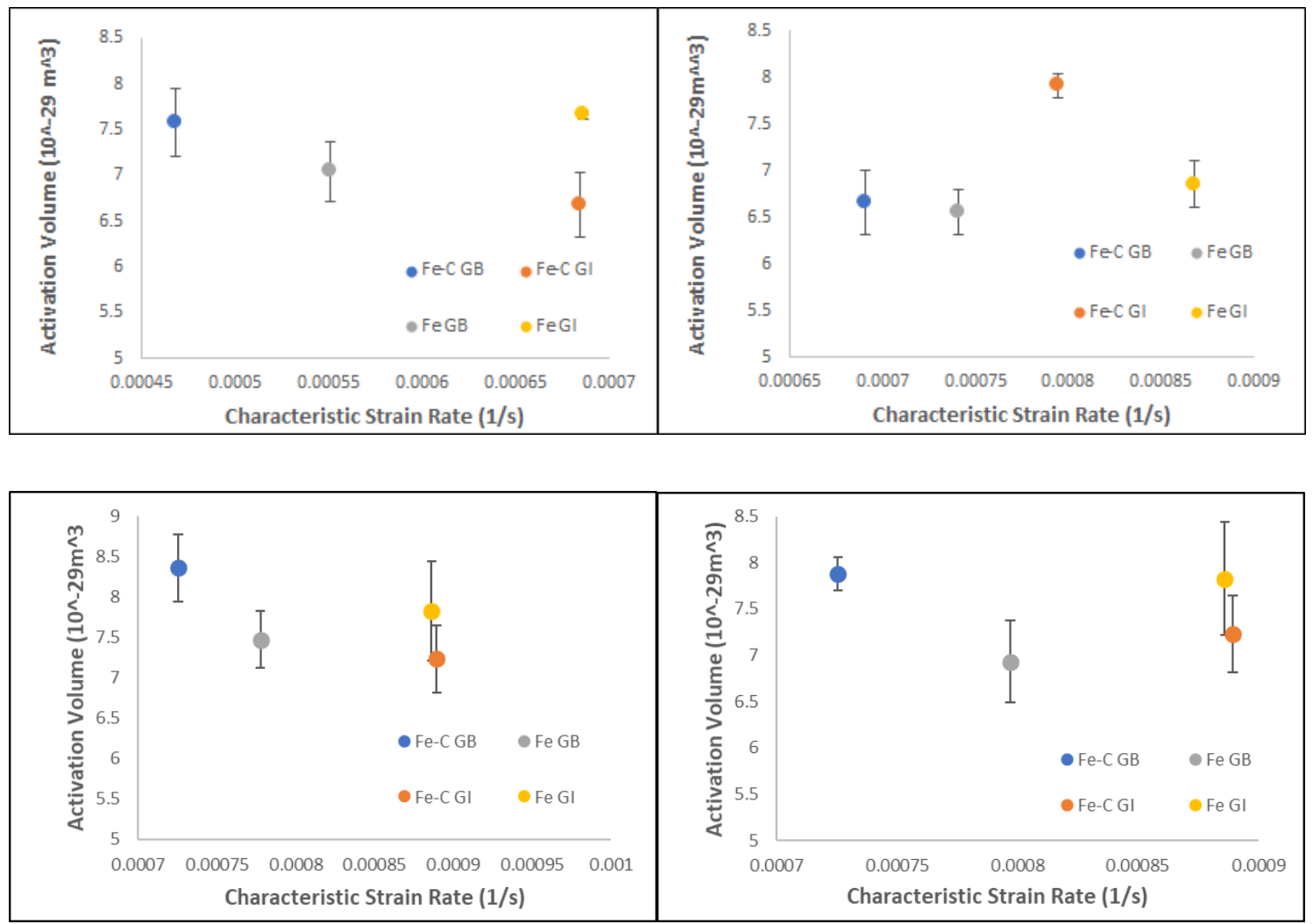

Figure 4.17. The Johnson-Cook analysis: activation volume vs characteristic strain rate for grain boundary and grain interior indentation with and without carbon, in (a) GB1, (b) GB2, (c) GB3, (d) GB4. The legend indicates grain boundary (GB) or grain interior (GI) 
in pure iron $(\mathrm{Fe})$ or carbon doped iron $(\mathrm{Fe}-\mathrm{C})$. The data is collected for indentation strain rate of $0.2 / \mathrm{s}$ in the loading segment.
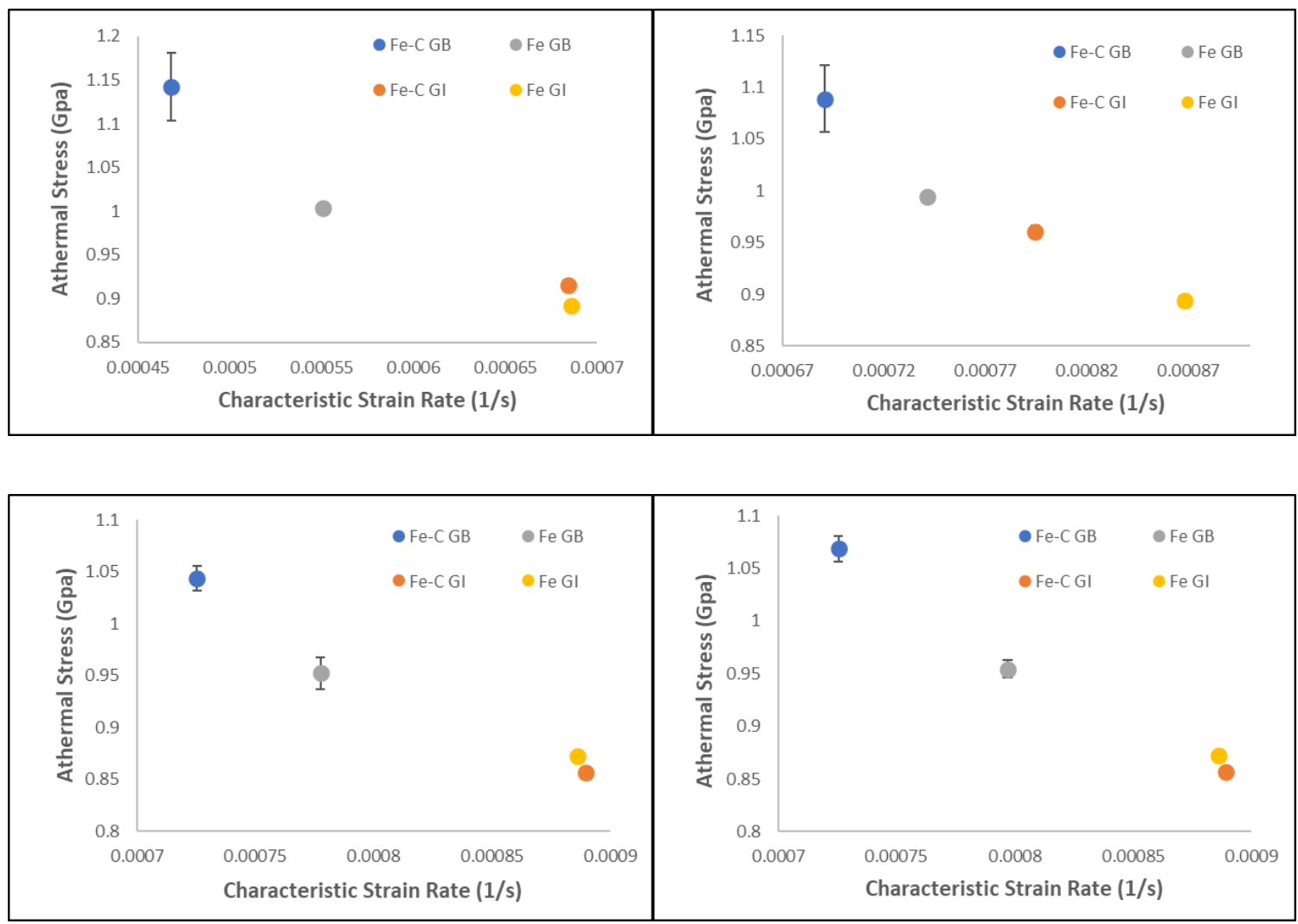

Figure 4.18. The Johnson-Cook analysis: Athermal stress vs characteristic strain rate for grain boundary and grain interior indentation with and without carbon, in (a) GB1, (b) GB2, (c) GB3, (d) GB4. The legend indicates grain boundary (GB) or grain interior (GI) in pure iron $(\mathrm{Fe})$ or carbon doped iron $(\mathrm{Fe}-\mathrm{C})$.

further determined using the Schoeck-Gibbs model (table 4.3). The following results present comparison of the physical properties associated with the grain boundary and grain interior indentation in the pure and carbon doped condition. 
The results for activation volume $(V)$ vs characteristic strain rate $\left(\dot{\varepsilon}_{o}\right)$ for all 4 general grain boundaries are presented in the figure (4.17) determined from constant load segments following deformation (indentation) at constant strain rate of $0.2 / \mathrm{s}$. It can be noticed that the activation volume doesn't appear to be influenced by the microstructural (in) homogeneity or carbon doping. As the activation volume at a given grain boundary and adjacent grain interior doesn't exhibit dependence on the carbon doping, it suggests that the mechanism of plastic deformation is unchanged. This is consistent with statements from other authors that "The Hall-Petch mechanism does not contribute to the activation volume" [64]. At the same time, the characteristic strain rate shows strong relationship with the chemistry and microstructural (in)homogeneity. The grain interior characteristic strain rate values are significantly higher compared with that at the grain boundaries. As shown in figure (4.18) a presence of solute atoms in the carbon doped samples further enhances the variation of characteristic strain rate with microstructure. While activation volume is being discussed, author would like to acknowledge that the activation volume estimated using the Johnson-Cook and Schoeck-Gibbs approach is an average measure of area swept by the dislocations during constant load hold segment. But activation volume evolution is examined using novel approaches in the appendix (4.A) which allows for variable activation volume during the hardness relaxation process. The figure (4.18) presents the qualitative relationship between athermal stress and characteristic strain rate for grain boundaries and grain interiors. These results suggest that the grain boundary back-stresses are higher compared to the grain interior athermal stresses. Moreover, carbon doping further increases the athermal stresses at the grain boundary and grain interior, although the effect is particularly noticeable at the grain boundary.

These results, particularly figure (4.18) requires further examination of the role of dislocation density and activation energy as specified in the equation (4.7). The increase in grain boundary back-stress with decreasing characteristic strain rate suggest that the mobile dislocation density may have decreased and/or the activation energy may have increased. The role of mobile dislocation density and activation energy can be examined utilizing 
work of Nix [59] and Smidt [36] respectively. The Nix model is presented in the appendix 4.B, where athermal stress or back-stresses are related to the mobile dislocation density through equation (4.B.4) and allows the calculation of the mobile dislocation density as a function of stress, athermal stress and total dislocation density. The results of this calculation of mobile dislocation density are shown in figure (4.19), where experimental values of stress and athermal stress are combined with the estimated total dislocation density of $\sim 10^{14} 1 / \mathrm{m}^{2}$. The effect of microstructural (in)homogeneity is evident where the estimated mobile dislocation density at the grain interior is higher than that at the grain boundaries. It is also apparent that the presence of solute affects the dislocation dynamics, significantly reducing the calculated mobile dislocation density at the grain boundary indentation in the presence of carbon doping and to some extent in the grain interior

Another parameter that may contribute to the observed grain boundary behavior as compared to the grain interior is the activation energy. for dislocations to overcome obstacles such as forest dislocations and grain boundaries. Typically it would be preferred to determine the activation energy using elevated temperature experiments. However, elevated temperature nano-indentation was not an option in this work. However, F. A. Smidt [36] has proposed an approach to measure activation energy using stress relaxation tests at different strain rate. This method is presented below:

Smidt relates activation volume and drop in hardness over stress relaxation duration $(\Delta \sigma)$ to determine activation energy as:

$$
V=\frac{1}{2} * \Delta g *\left(\tau_{p} * \Delta \sigma\right)^{-\frac{1}{2}}
$$

Where $\tau_{p}$ is the Peierls stress. Rearranging, 


$$
\log _{10} V=-\frac{1}{2} * \log _{10}(\Delta \sigma)-\frac{1}{2} *\left(\log _{10}\left(\frac{2 * \tau_{p}}{\Delta g}\right)\right.
$$
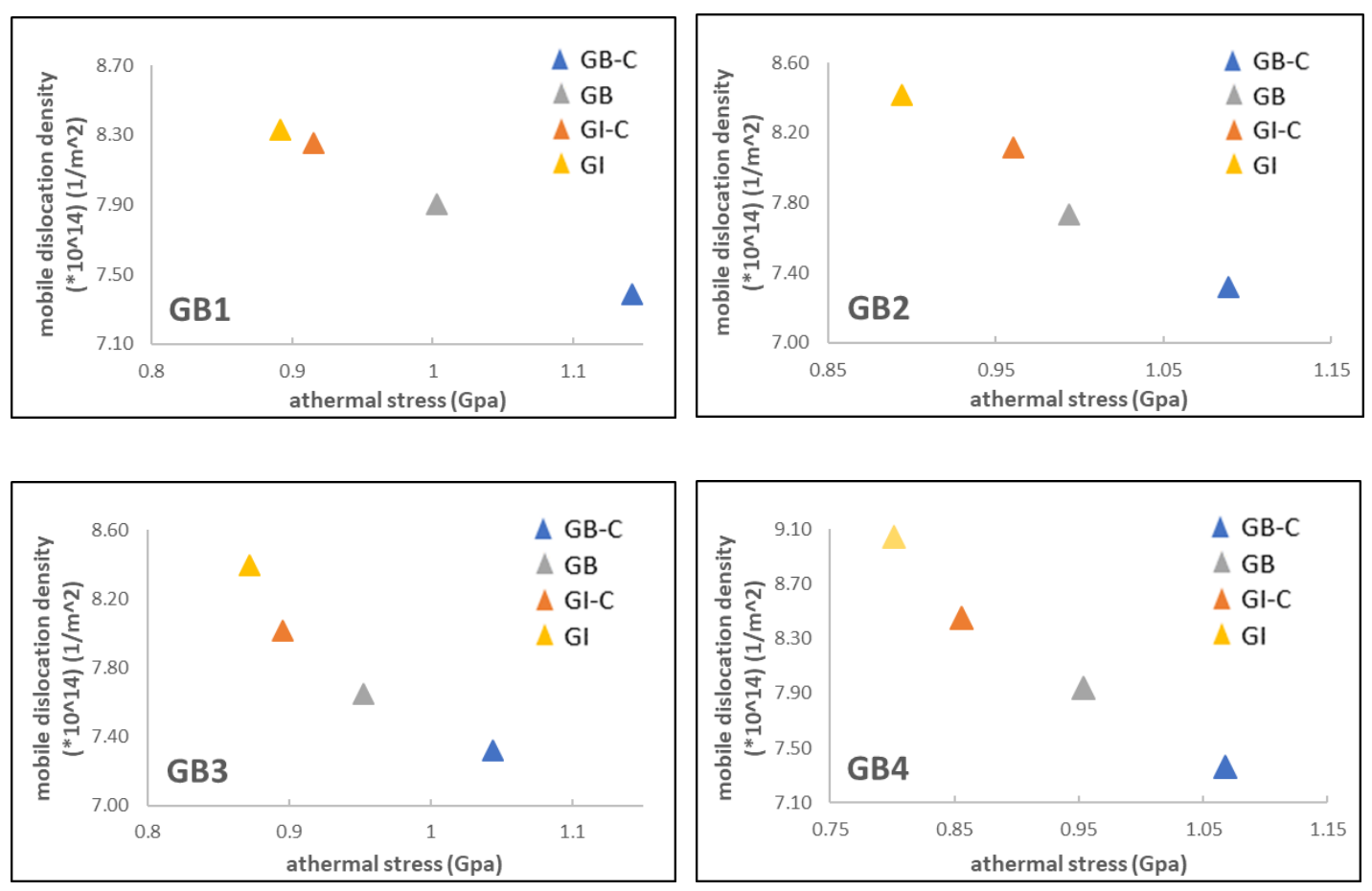

Figure 4.19. Mobile dislocation density vs athermal stress for grain boundary and grain interiors with and without carbon in (a) GB1, (b) GB2, (c) GB3, (d) GB4.

Although the experimental limitations allow only two activation volume and athermal stress data points for each grain boundary misorientation and chemistry, the linear form of equation 4.27 still allows for analysis. Activation energy (c) can be estimated using linear fitting and is a slope of $\log (\mathrm{V})$ vs $\log (\Delta \sigma)$ :

$$
c=2 * \tau_{p} / 10^{\wedge}(-2 *(\mathrm{y} \text { intercept }))
$$


Where, $\tau_{p}=0.11143 G p a,[65]$

The activation energy determined using equation (4.28) corresponds to the effective stress to be zero. The activation energy is determined for all the grain boundaries and grain interior in pure iron and carbon doped iron conditions, as presented in figure (4.20).

Author would like to acknowledge the apparent "scatter" in the data used for the activation energy and thus statistical analysis is performed as due. The statistical analysis is performed to estimate standard deviations and confidence interval for the data analyzed to measure activation energy. Using the "LINEST" function to estimate standard deviation associated with the slope and y-intercept of the linear fit. This data, particularly deviations in y-intercept are used to account for the standard deviation in activation energy. Based on this analysis, p-value and error bars are presented for the appropriate data.

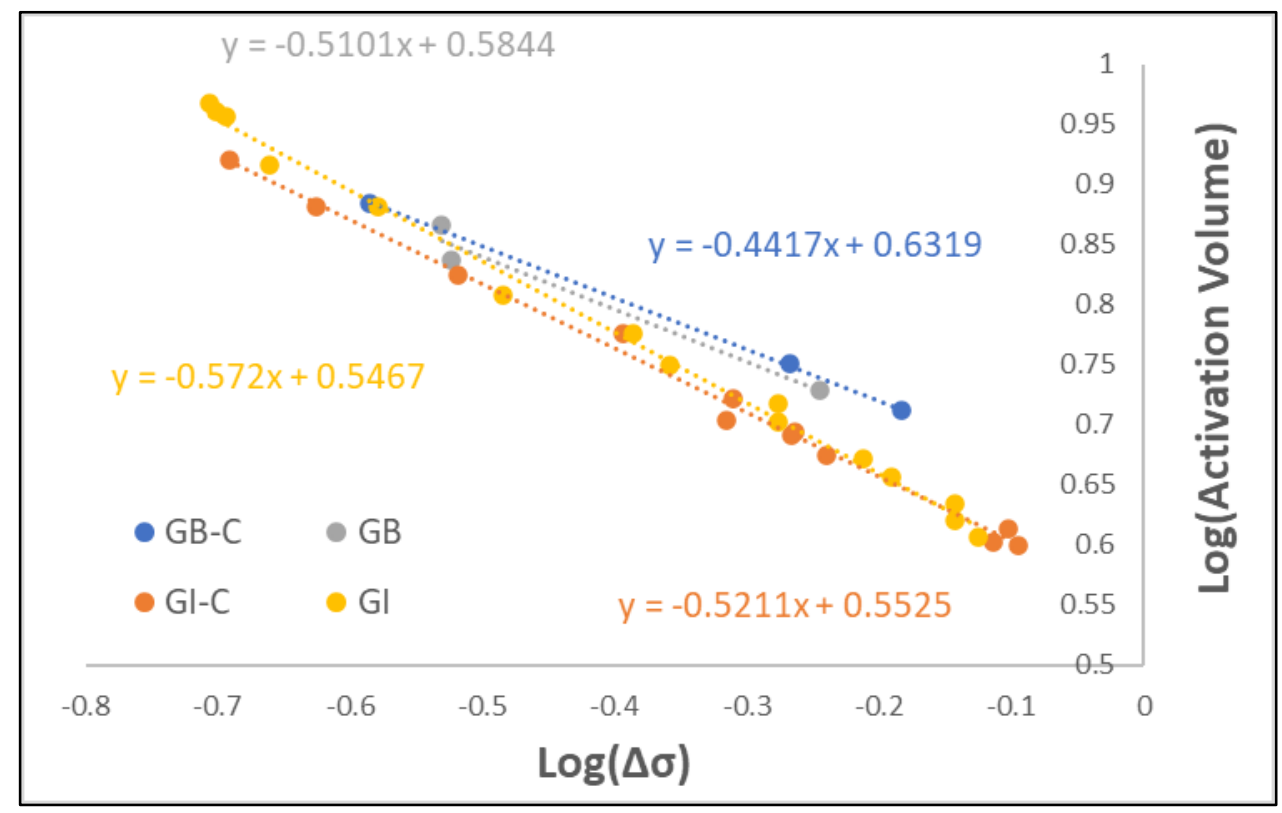

(a) 


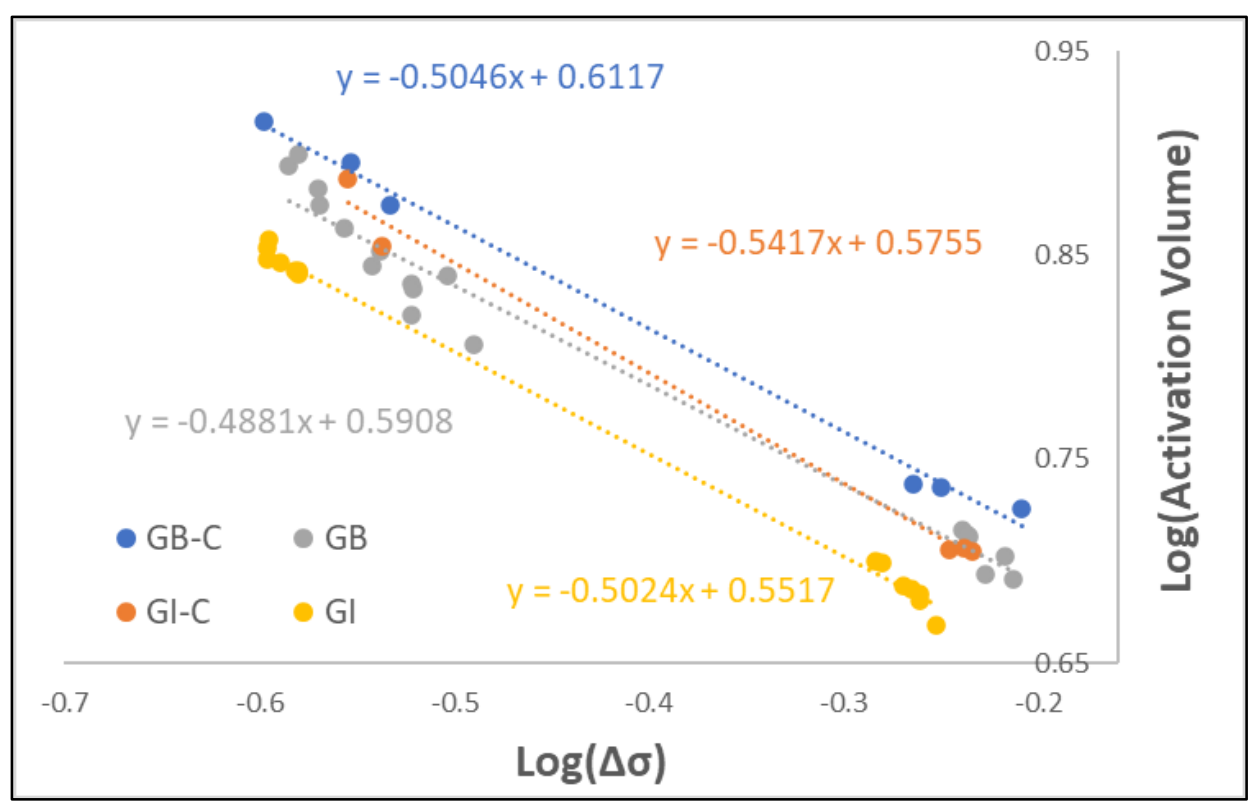

(b)

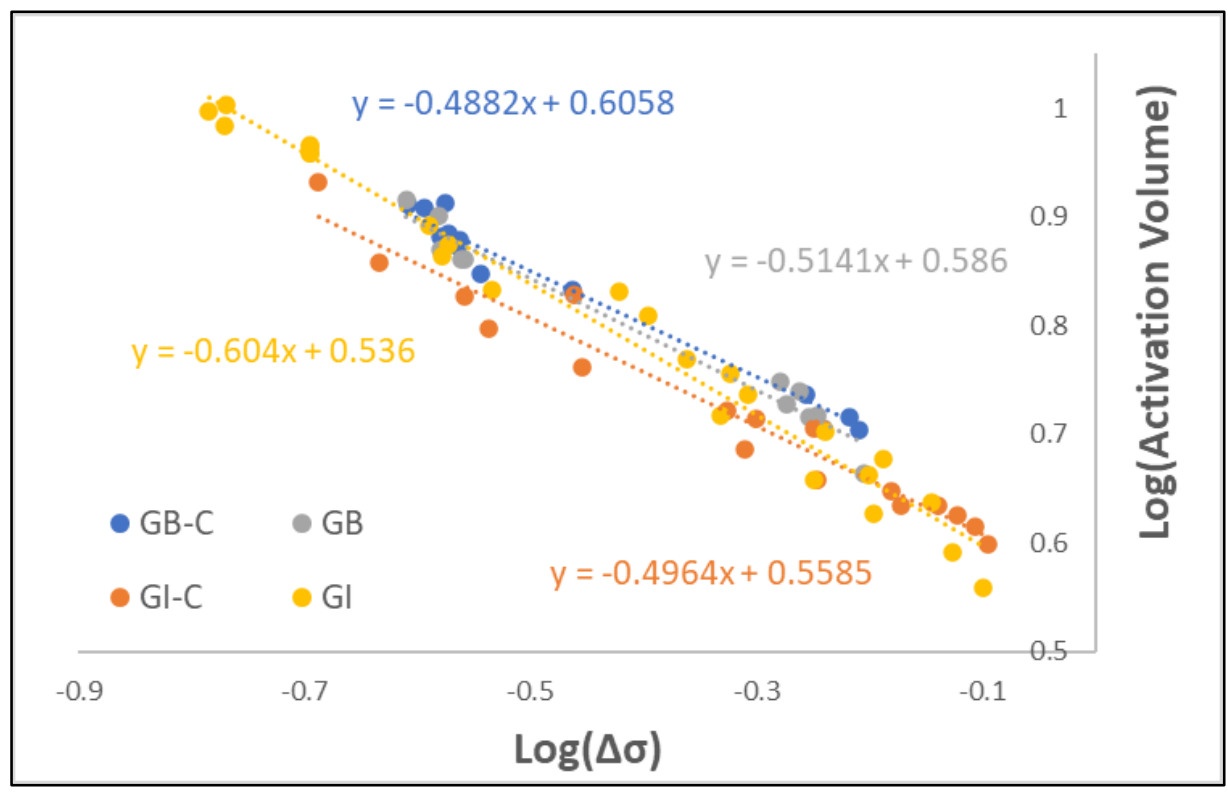

(c) 


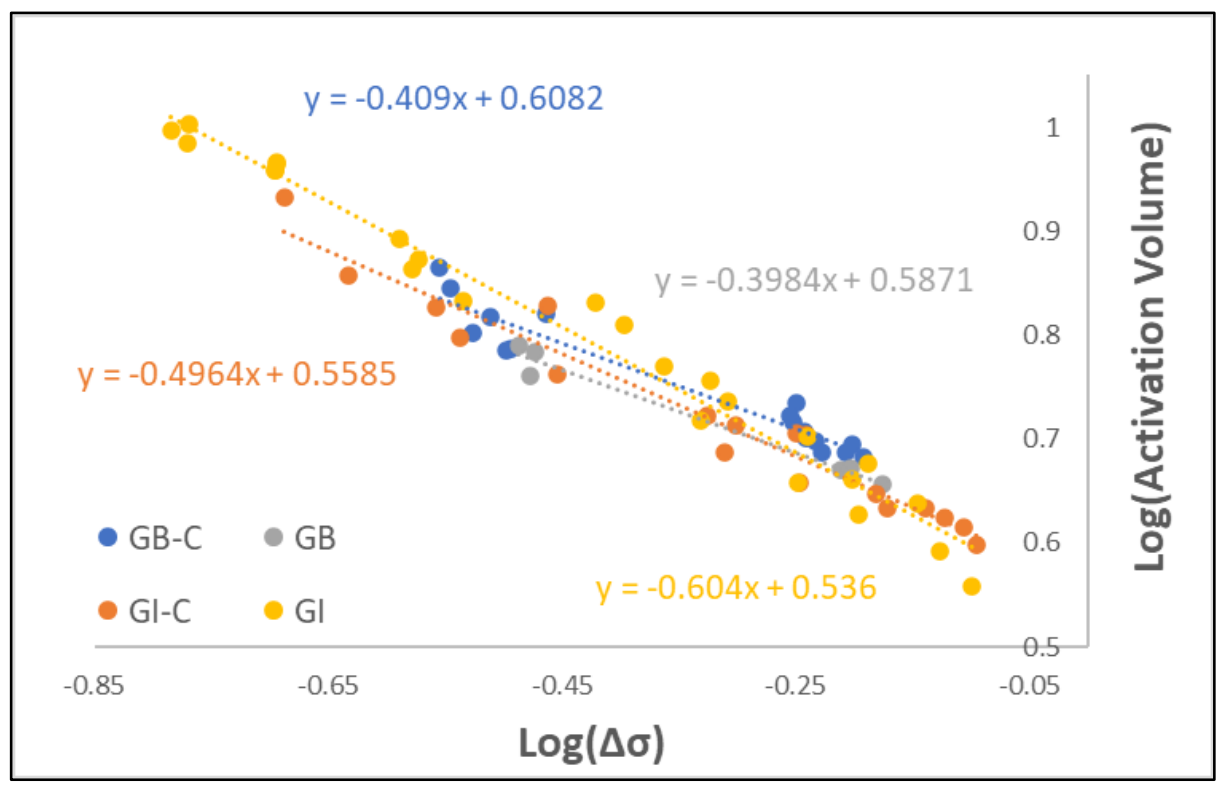

(d)

Figure 4.20. A log-log plot for activation volume vs hardness drop over constant load hold segment, where the activation energy can be determined using slope of the curve, (a) GB1, (b) GB2, (c) GB3, (d) GB4.

\begin{tabular}{|c|c|c|c|c|c|}
\hline \multicolumn{5}{|c|}{ Activation Energy Calculations - GB1 } \\
\hline \multirow{2}{*}{ Slip System } & \multirow{4}{*}{$\begin{array}{c}\text { Peierls } \\
\text { Stress (Gpa) }\end{array}$} & \multicolumn{4}{|c|}{ Activation Energy $\left({ }^{*} 10^{-20}\right)(\mathrm{J})$} \\
\cline { 3 - 6 } & & $\mathrm{GB}$ & $\mathrm{Gl}$ & $\mathrm{GB}$ & $\mathrm{GI}$ \\
\cline { 3 - 6 } & & 2.564077 & 2.350883 & 2.860428 & 2.38249 \\
\hline$\{110\}<111>$ & 0.11143 & 12.62153 & 11.57209 & 14.0803 & 11.72767 \\
\hline$\{112\}<111>$ & 2.7 & 28.11787 & 25.77997 & 31.36768 & 26.12657 \\
\hline$\{123\}<111>$ & 13.4 & 14.43449 & 13.23431 & 16.1028 & 13.41224 \\
\hline \multicolumn{2}{|c|}{ Average AE $\left({ }^{*} 10^{-20}\right)(\mathrm{J})$} & 0.900857 & 0.825954 & 1.004976 & 0.837058 \\
\hline \multicolumn{4}{|c|}{ Average AE $(\mathrm{eV})$} & &
\end{tabular}

Table 4.5. Activation energy for the grain boundary and grain interior with and without carbon for GB1. 


\begin{tabular}{|c|c|c|c|c|c|}
\hline \multicolumn{6}{|c|}{ Activation Energy Calculations - GB2 } \\
\hline \multirow{3}{*}{ Slip System } & \multirow{3}{*}{$\begin{array}{c}\text { Peierls } \\
\text { Stress (Gpa) }\end{array}$} & \multicolumn{4}{|c|}{ Activation Energy $\left(* 10^{-20}\right)(\mathrm{J})$} \\
\hline & & \multicolumn{2}{|c|}{ Pure iron } & \multicolumn{2}{|c|}{ carbon doped iron } \\
\hline & & GB & Gl & GB & GI \\
\hline$\{110\}<111>$ & 0.11143 & 2.602142 & 2.378105 & 2.73043 & 2.512066 \\
\hline$\{112\}<111>$ & 2.7 & 12.8089 & 11.70609 & 13.44039 & 12.36551 \\
\hline$\{123\}<111>$ & 13.4 & 28.5353 & 26.07849 & 29.94211 & 27.54751 \\
\hline \multicolumn{2}{|c|}{ Average $\mathrm{AE}\left(* 10^{-20}\right)(\mathrm{J})$} & 14.64878 & 13.38756 & 15.37098 & 14.14169 \\
\hline \multicolumn{2}{|c|}{ Average $\mathrm{AE}(\mathrm{eV})$} & 0.91423 & 0.835518 & 0.959303 & 0.882583 \\
\hline
\end{tabular}

Table 4.6. Activation energy for the grain boundary and grain interior with and without carbon for GB2.

\begin{tabular}{|c|c|c|c|c|c|}
\hline \multicolumn{5}{|c|}{ Activation Energy Calculations - GB3 } \\
\hline \multirow{2}{*}{ Slip System } & \multirow{4}{*}{$\begin{array}{c}\text { Peierls } \\
\text { Stress (Gpa) }\end{array}$} & \multicolumn{3}{|c|}{ Activation Energy $\left({ }^{*} 10^{-20}\right)(\mathrm{J})$} \\
\cline { 3 - 6 } & & $\mathrm{GB}$ & $\mathrm{GI}$ & $\mathrm{GB}$ & $\mathrm{GI}$ \\
\cline { 3 - 6 } & & 2.573541 & 2.293671 & 2.693587 & 2.415634 \\
\hline$\{110\}<111>$ & 0.11143 & 12.66811 & 11.29047 & 13.25903 & 11.89082 \\
\hline$\{112\}<111>$ & 2.7 & 28.22165 & 25.15257 & 29.53809 & 26.49003 \\
\hline$\{123\}<111>$ & 13.4 & 14.48777 & 12.91224 & 15.16357 & 13.59883 \\
\hline \multicolumn{2}{|c|}{ Average AE $\left(* 10^{-20}\right)(\mathrm{J})$} & 0.904182 & 0.805853 & 0.946358 & 0.848703 \\
\hline \multicolumn{2}{|c|}{ Average AE $(\mathrm{eV})$} & &
\end{tabular}

Table 4.7. Activation energy for the grain boundary and grain interior with and without carbon for GB3. 


\begin{tabular}{|c|c|c|c|c|c|}
\hline \multicolumn{6}{|c|}{ Activation Energy Calculations - GB4 } \\
\hline \multirow{3}{*}{ Slip System } & \multirow{3}{*}{$\begin{array}{c}\text { Peierls } \\
\text { Stress (Gpa) }\end{array}$} & \multicolumn{4}{|c|}{ Activation Energy $\left(* 10^{-20}\right)(\mathrm{J})$} \\
\hline & & \multicolumn{2}{|c|}{ Pure iron } & \multicolumn{2}{|c|}{ carbon doped iron } \\
\hline & & GB & GI & GB & GI \\
\hline$\{110\}<111>$ & 0.11143 & 2.580067 & 2.293671 & 2.708514 & 2.415634 \\
\hline$\{112\}<111>$ & 2.7 & 12.70024 & 11.29047 & 13.33251 & 11.89082 \\
\hline$\{123\}<111>$ & 13.4 & 28.29322 & 25.15257 & 29.70177 & 26.49003 \\
\hline \multicolumn{2}{|c|}{ Average AE $\left(* 10^{-20}\right)(\mathrm{J})$} & 14.52451 & 12.91224 & 15.2476 & 13.59883 \\
\hline \multicolumn{2}{|c|}{ Average AE (eV) } & 0.906475 & 0.805853 & 0.951603 & 0.848703 \\
\hline
\end{tabular}

Table 4.8. Activation energy for the grain boundary and grain interior with and without carbon for GB4.

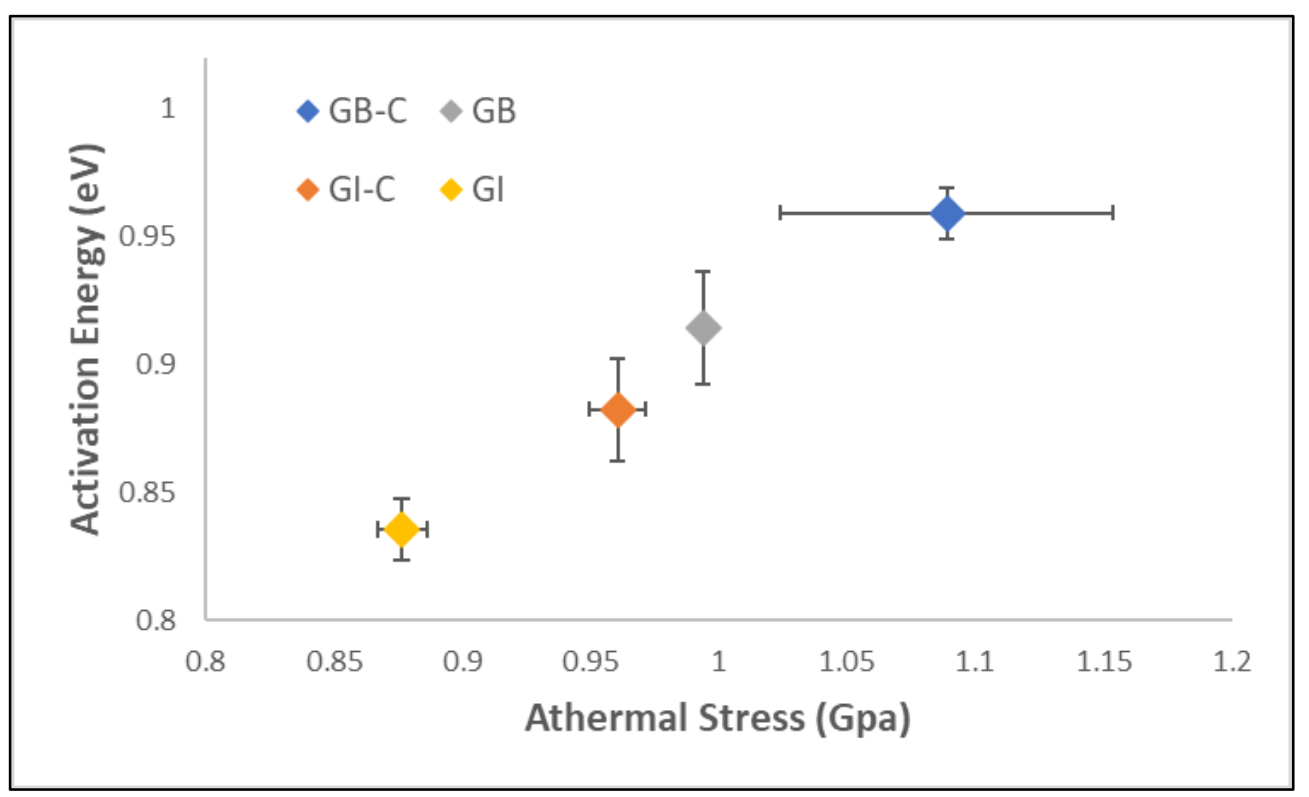

(a) 


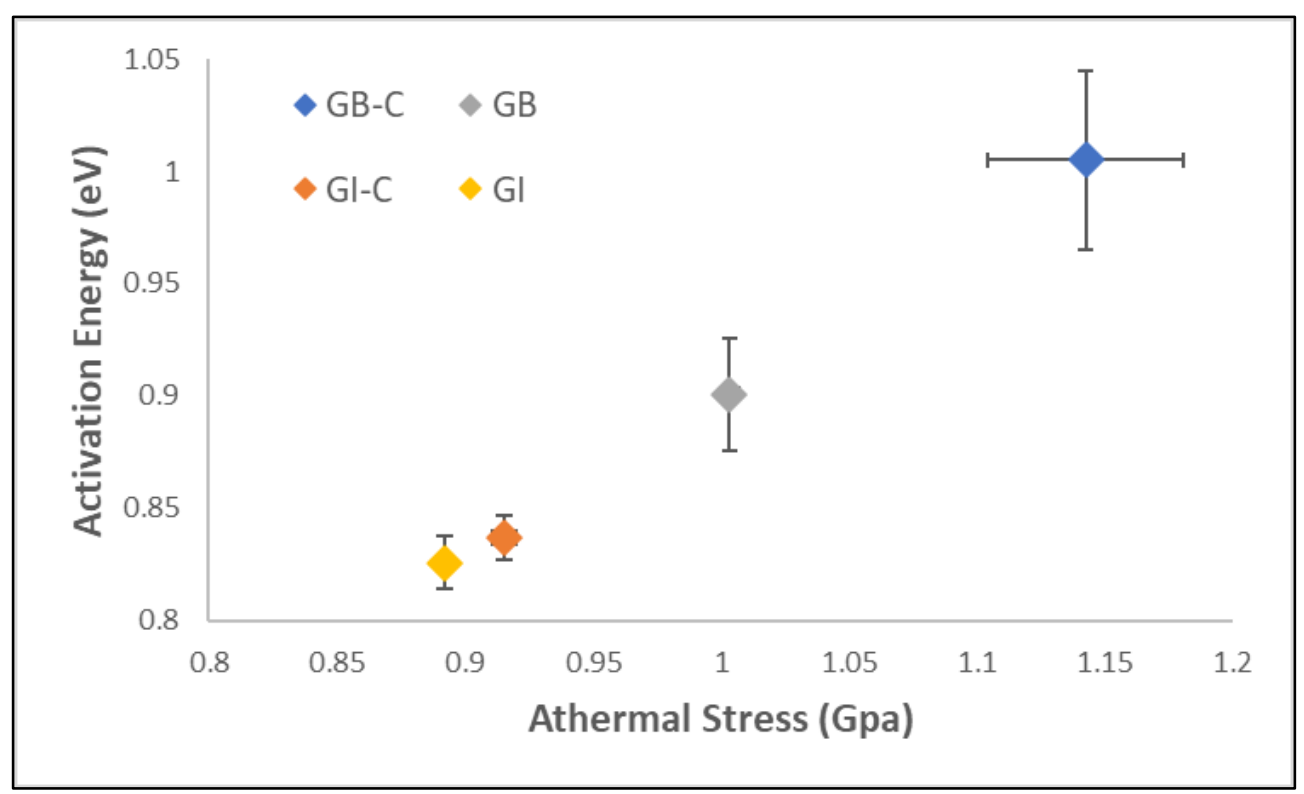

(b)

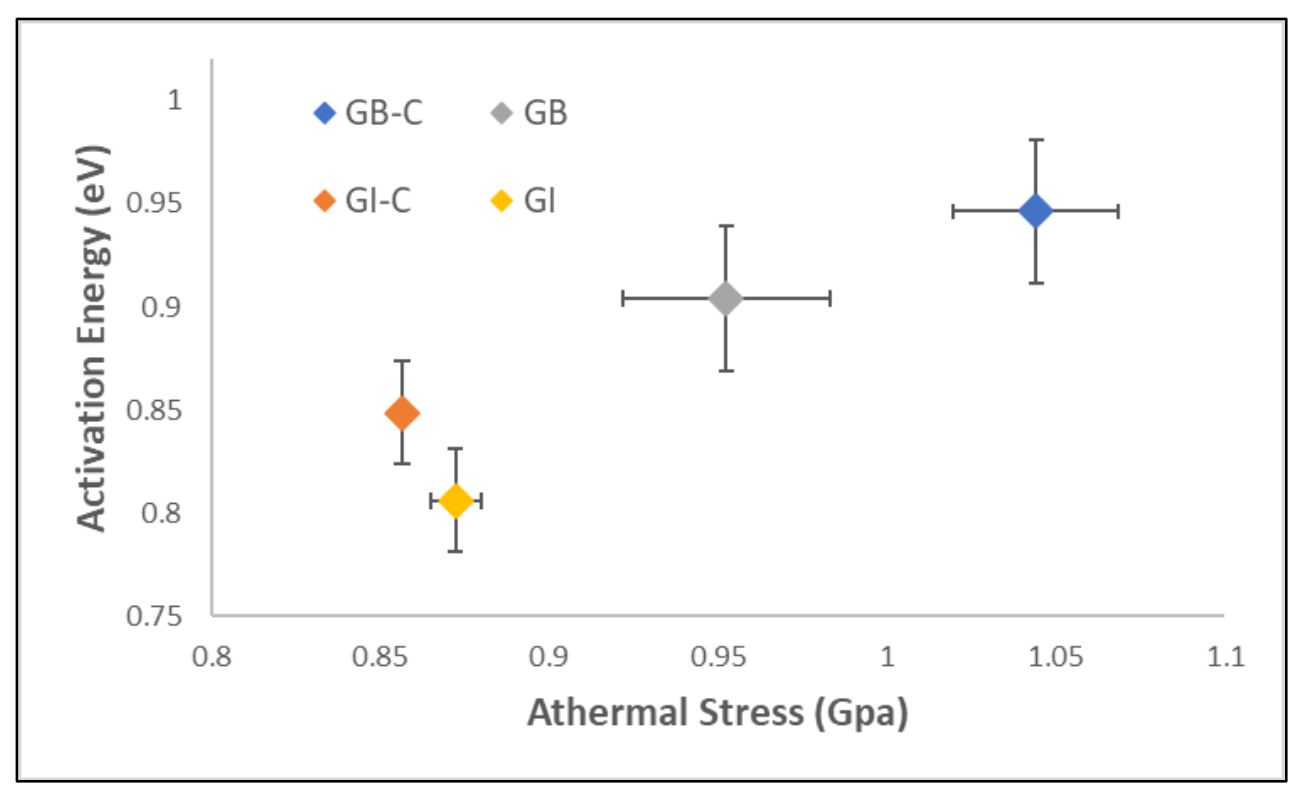

(c) 


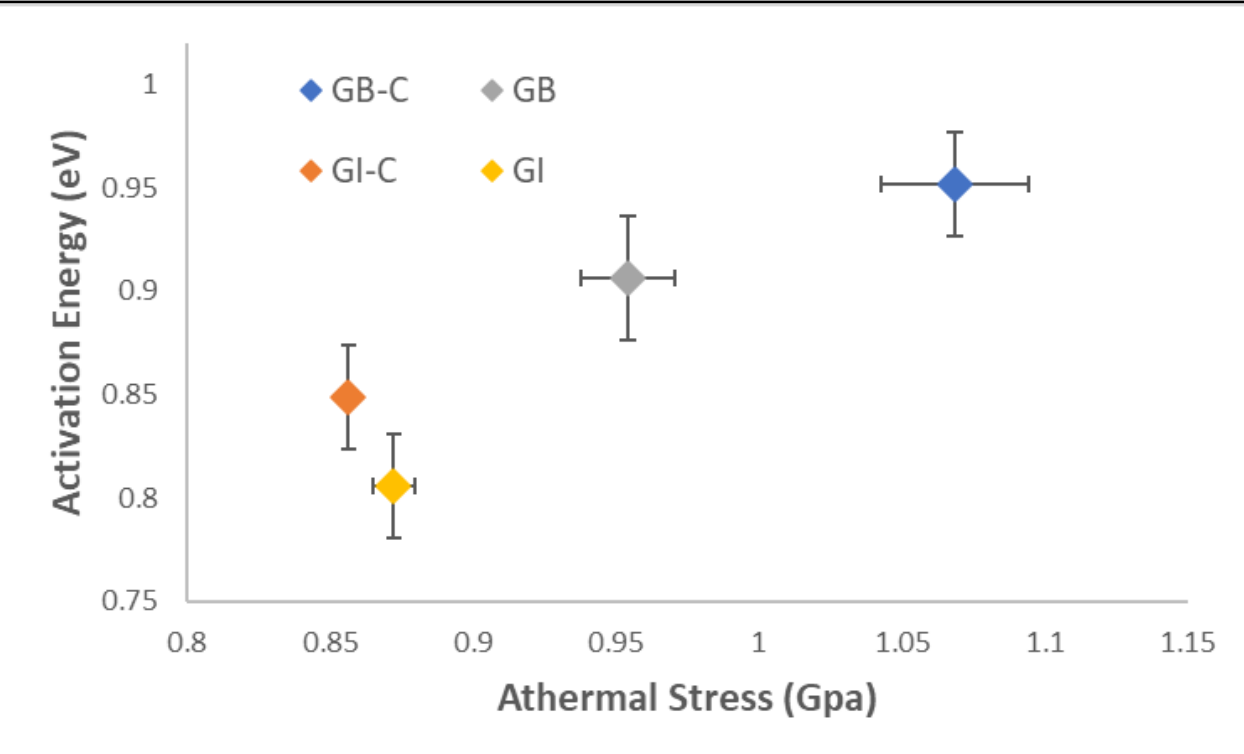

(d)

Figure 4.21. Activation energy vs athermal stress based on statistical analysis GB data is different than GI data for (a) GB1, (b) GB2, (c) GB3, (d) GB4.

From the activation energy estimations, it can be seen that the grain boundary offers higher resistance to the dislocation motion as compared to the grain interior. The carbon doping effect is also evident where addition of carbon enhances resistance to the dislocation motion. As solute segregation increases the stability of the grain boundary through energy minimization, the effect is manifested through higher athermal stresses.

As mentioned in the introduction, this work further utilizes Evans-Kocks model to examine carbon doping effects through critical stress measurements. The solute atoms in the grain interior as well as at the grain boundary act as a pinning sources affecting dislocation flow. Using the strain rate-stress relationship presented by Evan-Kocks, JC model parameters are examined in terms of critical stress. 
As shown in figure (4.22), the grain interior in pure and carbon doped condition is examined to study combined effect of strain rate induced dislocation density and carbon doping. The critical stress appears to increase with the indentation strain rate. Higher the applied stress, more dislocations are introduced in the material under indentation process. This increase in dislocation density (or defect structures) as a result of increased applied stress (or indentation strain rate) may result in proportional increase in the critical stress required to overcome obstacles.

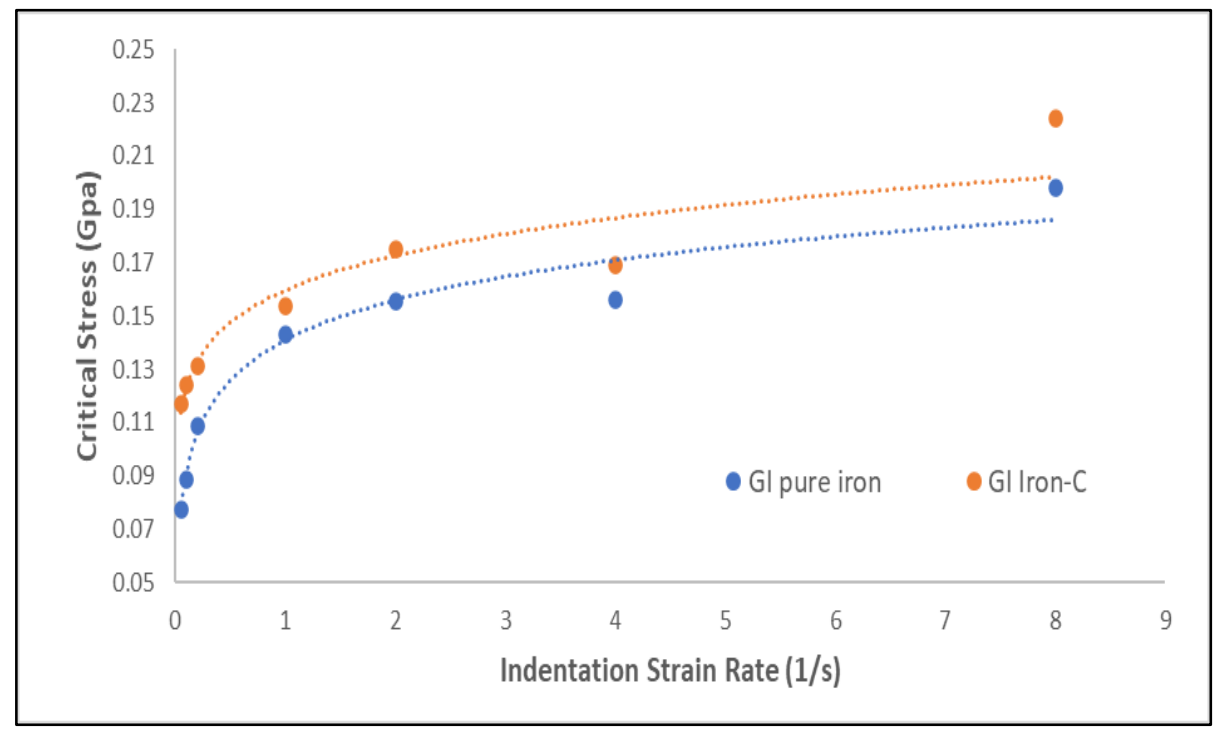

Figure 4.22. Evans-Kocks model-based critical stress as a function of indentation strain rate for the pure iron and carbon doped iron grain interior for GB1.

Additionally, results shown in figure (4.22) also highlight effect of solute doping wherein the obstacle strength for carbon doped grain interior appears to be higher than its pure iron counterpart. The solute doping contribution doesn't appear to be as significant as the 
mobile dislocation density in terms of affecting activation volume in different grain boundary vs grain interior conditions.

The Evans-Kocks analysis is further extended to the grain boundary indentations with and without carbon. The results presented in the figure (4.23), suggest that the critical stress associated with the grain boundary indentations is higher than the grain interior and comparing with and without carbon grain boundary indentations, solute doping effects are notably higher. The solute effects in the grain interior and grain boundary can be considered in the context of Cottrell atmosphere and Excess free volume, respectively. In the grain interior, solute atoms give rise to the Cottrell atmosphere. The presence of carbon atoms distorts the lattice and create residual strain field in the vicinity affecting dislocation flow. At the grain boundary, the discontinuity in crystal periodicity results in excess free

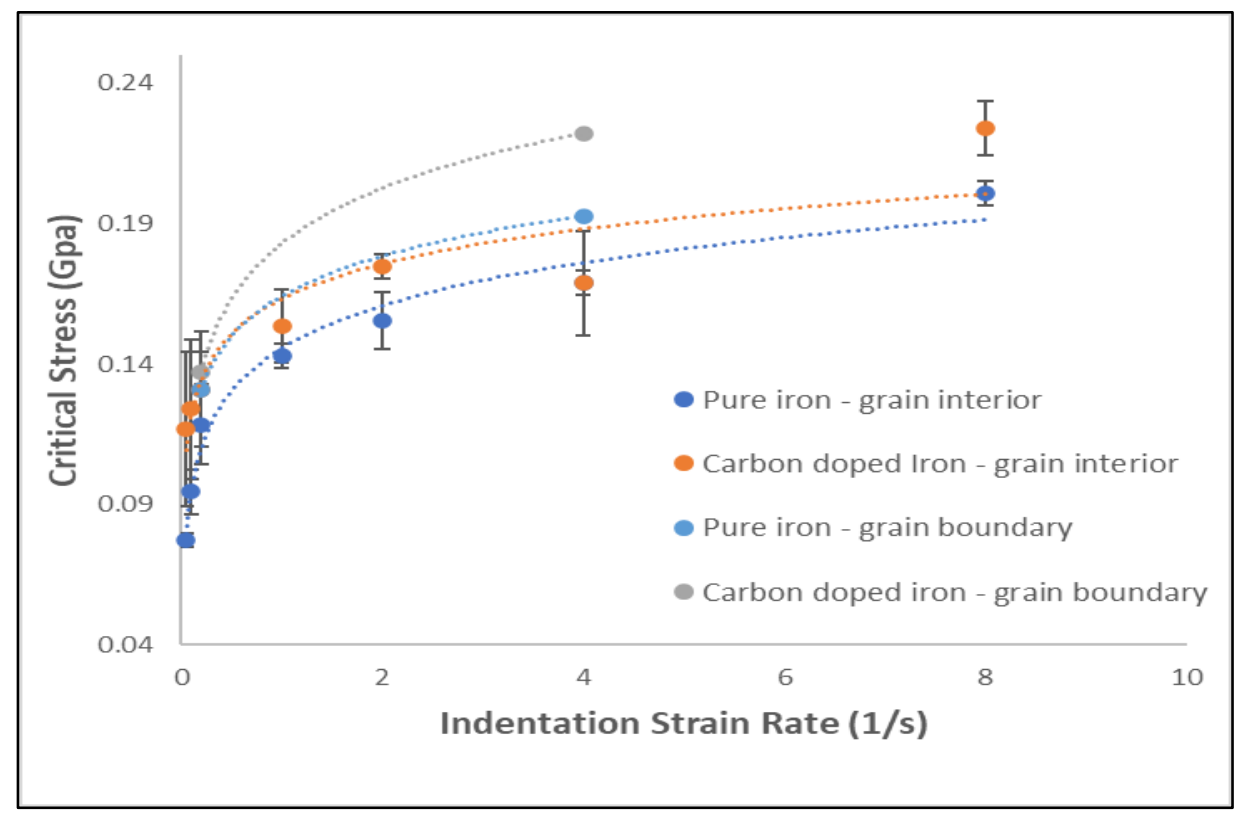

Figure 4.23. The critical stress measured using Evans-Kocks model against the indentation strain rate, for grain boundaries and grain interior, with and without carbon, for GB1. 
volume in the grain boundary plane [66-68]. The energy minimization principle drives solute segregation at the grain boundary that results in interstitial atoms occupying free volume in the grain boundary vicinity [66-68]. The solute segregation enhances resistance of the grain boundary to further facilitate nucleation or emission of dislocations, manifested through higher critical stress [66-68] as shown in figure (4.22).

\subsubsection{Local GB vs Remote GB indentations: JC and SGP model}

A description of the indentation geometry relative to the grain boundary, termed 'local' and 'remote' has been introduced in chapter 3 [30]. As shown in the figure (4.24), both types of these grain boundary indentations exhibit hardening relative to the adjacent grain interior. But the difference in hardening behavior of local and remote indentations was obvious through a unique hardness-depth response and associated extrinsic indentation size effect. The mechanical response of the local geometry was proposed to correspond to the Ashby theory of grain boundary hardening, while the remote geometry was suggested to exhibit a mechanical response consistent with the Hall-Petch pile-up mechanism of initial yielding at the grain boundary. In this work, the difference in the local and remote indentation is re-examined through the Johnson-Cook (JC) model interpretation of the constant load hardness relaxation data with particular emphasis on the grain boundary chemistry affected by carbon doping.

Additionally, this work utilizes SGP model interpretation for the local indentation analysis introduced in the chapter 3 [30], where extrinsic indentation size effect (ISE) associated with the local buildup of geometrically necessary dislocations at the interface due to plastic strain incompatibility of adjacent grain interiors is examined against the experimental results. As this model is purely based on the grain boundary geometry, it is used here to examine grain boundary chemistry effects on the local GB indentations. 


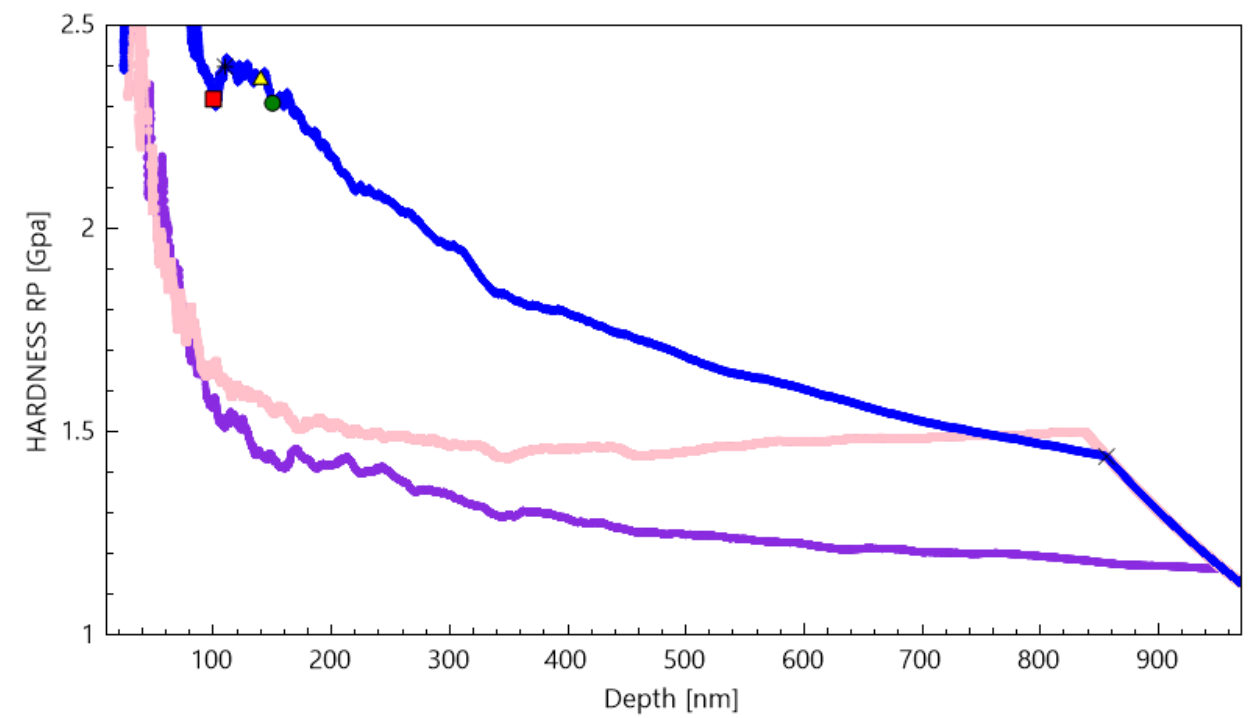

Figure 4.24. A representative hardness-depth curves for the local GB (blue), remote GB (pink) and adjacent grain interior (purple) indentation.

1. The Johnson-Cook (JC) analysis of local and remote GB indentations: pure iron and carbon doped iron

The constant load hardness relaxation behavior of local and remote indentations in pure and carbon doped conditions is shown in figure (4.25). It is important to note that the indentations used in these figures, and analysis in general, are chosen in a way that the indentation position relative to the grain boundary, in with and without carbon conditions, are similar.

The hardness-strain rate responses of local and remote indentations are further examined using JC model. The physical parameters such as activation volume, athermal stress and characteristic strain rates are compared for both indentation geometries in pure and carbon doped iron. 


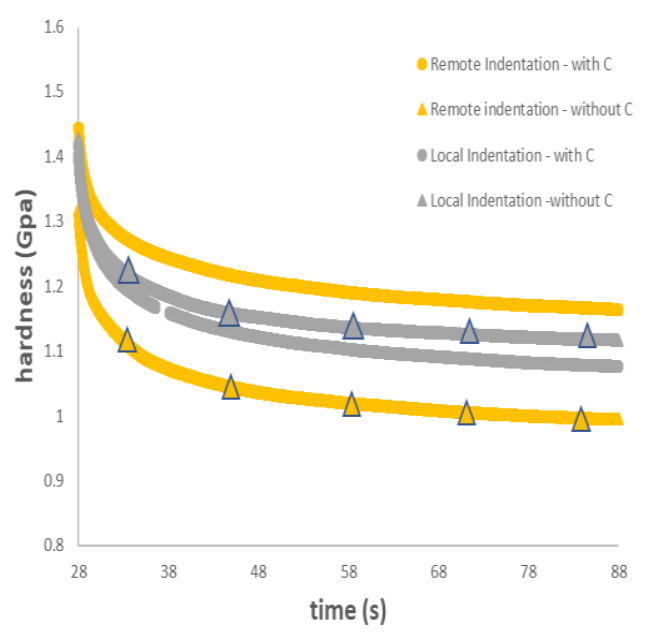

(a)

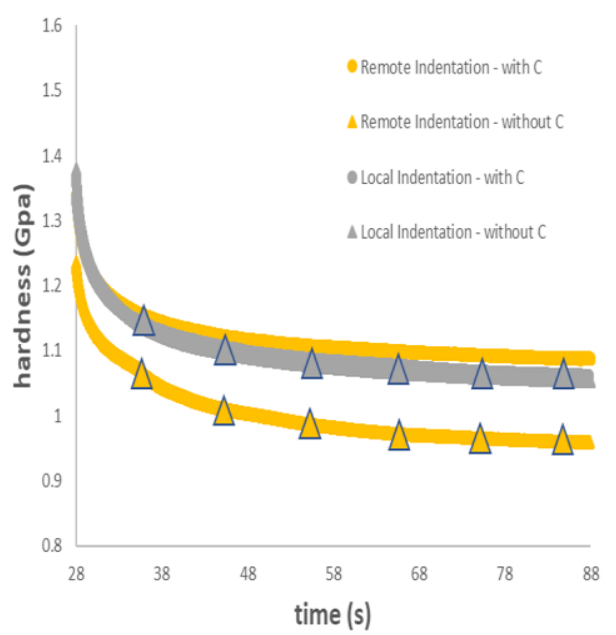

(b)

Figure 4.25. A representative hardness-time plot, Comparing similarly positioned local and remote GB indentation, where the remote indentation results are affected by the carbon doping whereas local indentation behavior appears independent of the grain boundary chemistry for (a) GB1 and (b) GB2.

The figure (4.26) presents athermal stress vs characteristic strain rate for the two indentation geometries with and without carbon doping. Comparing remote indentation results, it is observed that the athermal stress (or back-stress) associated with the indentation in carbon doped condition appear higher than the pure iron remote indentations. Whereas corresponding characteristic strain rate is lower than its pure iron counterpart. As mentioned in equation (4.7), the characteristic strain rate is a directly proportional to the mobile dislocation density and indirectly proportional to the activation energy. So, the remote indentation results may suggest that dislocation pinning effect may have enhanced in the presence of carbon atoms at the grain boundary and/or the activation energy for the dislocation transmission across the grain boundary has increased. 


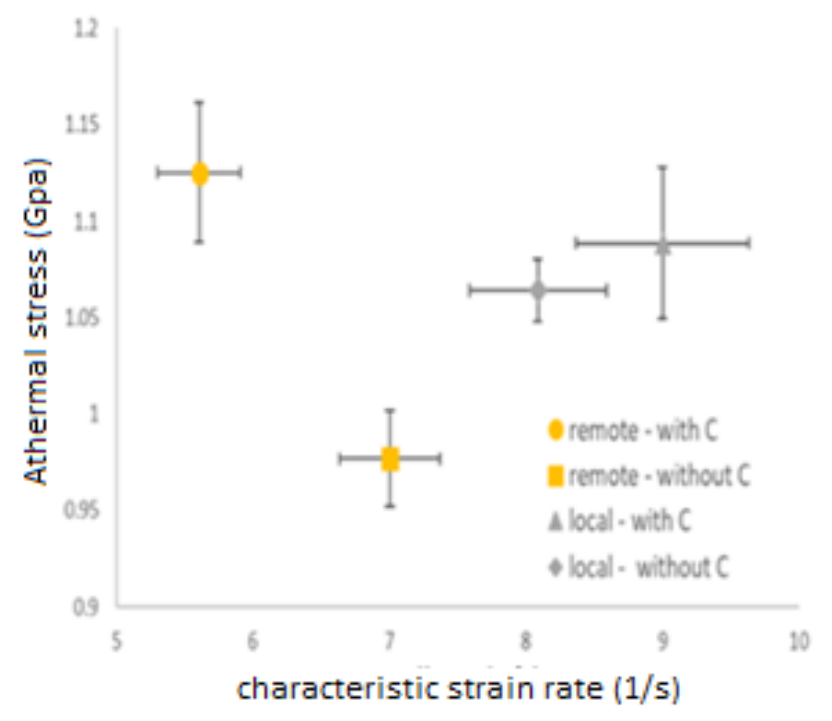

(a)

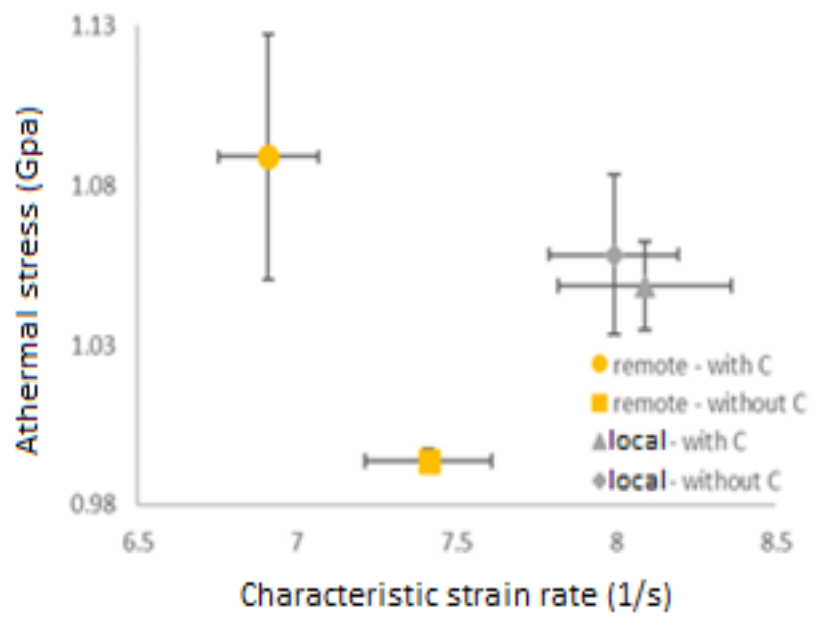

(b)

Figure 4.26. Athermal stress vs characteristic strain rate for local and remote GB indentation, where local GB indentation data for with and without carbon is statistically indistinguishable unlike remote GB indentations with and without carbon, for (a) GB1 and (b) GB2. 
On the other hand, the results for the local indentations in pure and carbon doped iron are statistically indistinguishable, suggesting the chemistry changes at the grain boundary have little to no effect on the grain boundary hardening mechanism.

2. The Strain Gradient Plasticity (SGP) model analysis of local GB indentations - pure iron and carbon doped iron

The strain gradient plasticity concepts are utilized to study local mechanical response of grain boundary during simultaneous deformation of the adjacent grain interior. The hardness at the grain boundary is provided as:

$$
H=\left[H_{o}\left(1+\frac{h^{*}}{2 f^{3} h}\right)\right]_{\text {intrinsic }}+\left[\left.\overline{\sigma_{(g b)}}\right|_{x=\frac{\pi}{(2 \omega)}}\right]_{\text {extrinsic }}
$$

Where first part of the equation represents contribution of grain interior towards the indentation size effect, whereas second part accounts for the strain and strain rate effects associated with the grain boundary deformations. The well-annealed grain interior is associated with the minimal indentation size effect. The grain boundary deformation is affected by the strain incompatibly of adjacent grains which suffer differing amount of plastic displacement, during indentation. The geometrically necessary dislocations are introduced in the vicinity of the grain boundary to account for the difference in the plastic displacements, giving rise to the strain gradient between the grain boundary and grain interior.

The extrinsic ISE contribution to the hardness is modeled as: 


$$
\left[\left.\overline{\sigma_{(g b)}}\right|_{x=\frac{\pi}{(2 \omega)}}\right]_{\text {extrinsic }}=\left(\frac{0.12 n H_{o} l_{1} \Delta m}{h}\right)+0.02 n \beta \Delta m
$$

Where, $l_{1}$ - characteristic length, $\Delta m$-Schmid factor, $\beta$ - hardening modulus,

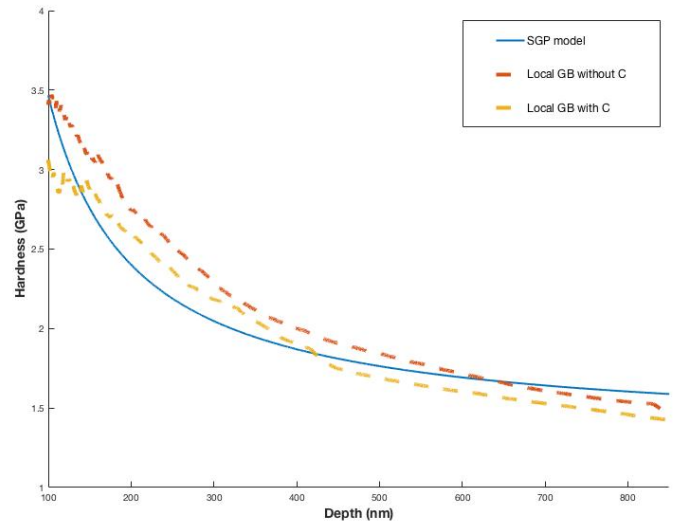

(a)

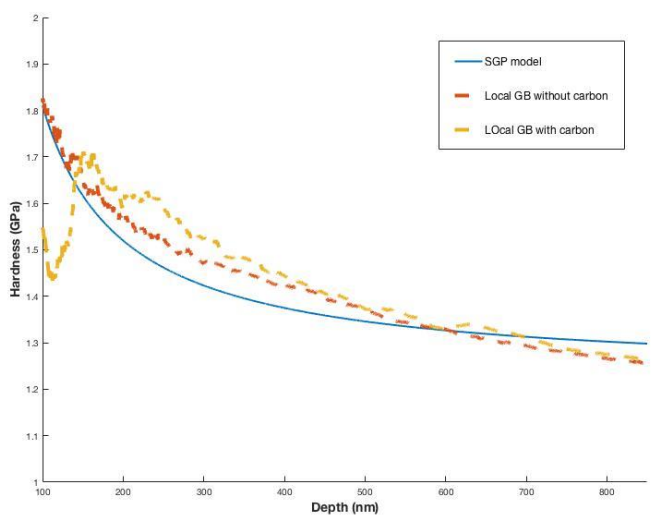

(b)

Figure 4.27. Strain Gradient Plasticity model fitting for local GB indentations with and without carbon for (a) GB1, (b) GB2 
The two fitting parameters, $\left(n H_{o} l_{1} \Delta m\right)$ and $(n \beta \Delta m)$, used in this analysis are interpreted as a measure of the grain boundary strength and mechanically induced interface energy during deformation.

As shown in the figure (4.27), the local indentation experimental results for with and without carbon conditions are plotted against the SGP fit curve, to determine fitting parameters. The results are presented for GB1 and GB2.

From the results shown in the figure (4.27), the local indentations with and without carbon doping hardness-depth profiles do match very closely and so, can be fitted well with the fitting parameters mentioned in the table (4.9). These results suggest that the hardening associated with the local indentation geometry is independent of the grain boundary chemistry effects.

\begin{tabular}{|c|c|c|}
\hline GB & $n H_{o} l_{1} \Delta m(\mathrm{Gpa} . \mathrm{nm})$ & $n \beta \Delta m(\mathrm{Gpa})$ \\
\hline GB1 & 125.0 & 12.0 \\
\hline GB2 & 45.0 & 7.0 \\
\hline
\end{tabular}

Table 4.9. SGP fitting parameters for local indentations on GB1 and GB2 for with and without carbon conditions. The fitting parameters are same for with and without carbon data, as shown in the figure 4.27 .

The currently accepted ideas of grain boundary strengthening via solute segregation appear to consider two models: Alloyed layer model and 'grain boundary excess free volume' model. The alloyed layer model was first envisioned by R. W. Armstrong [69]. The model treats solute segregated grain boundary region as a considerably thick (on the order of microns) alloyed layer of base metal and solute atoms. There are various studies [69-71] that have utilized this model where bulk sample experimental results indicated that 
the alloyed region exhibits different modulus and burger vector compared to the pure metal grain interior. It is suggested that the hardening response observed due to solute segregation is due to conventional alloy strengthening mechanism and solute atoms effectively enhance ability to store forest dislocations in the vicinity of the grain boundary resulting in the 'hardening layer'. On the other hand, the molecular dynamic approach, which can examine length scales, that are currently beyond experimentally attainable, seem to support utilization of excess free volume at the grain boundary to explain grain boundary strengthening in solute doped grain boundaries [66-68]. In this model, the solute atoms segregated at the grain boundary appear to fill in voids i.e. 'free volume' formed as a result of crystallographic misalignment across the grain boundary. This doesn't affect the grain boundary structure but enhances strength by stabilizing the grain boundary region. The molecular dynamic studies have studies dislocation-grain boundary interaction and resulting changes in grain boundary structure upon dislocation emission. These studies have indicated that the solute doping at grain boundary doesn't affect the slip geometry but reduces free spaces. It is proposed that the solute would occupy free volumes at the grain boundary resulting in strengthening of grain boundary against dislocation emission without apparent change in the grain boundary structure.

The grain boundary strengthening mechanism envisioned by Ashby and Taylor introduces concept of geometrically necessary dislocations that are required to accommodate for the difference in plastic displacement across grain boundary. In response to the applied stress, deformation of adjoining grains is constraints by geometry as a result of slip geometry difference. As a result, change in grain boundary chemistry, which doesn't affect slip mismatch across the boundary, is not expected to affect the grain boundary hardening mechanism. Also, in this mechanism interface vicinity is flooded with geometrically necessary dislocations to compensate for the plastic strain incompatibility. Such observations are evident from the Johnson-Cook, where athermal stress vs characteristic strain rate response of local indentation with and without carbon is statistically indistinguishable. Further SGP model confirms that the hardening observed in 
case of pure and doped carbon conditions, can be fitted with the same SGP response, which accounts for the extrinsic ISE contribution of grain boundary. Additional, supporting observation is through comparison of characteristic strain rate values of local indentation vs remote indentations i.e. $\dot{\varepsilon_{o}}$ is considerably higher than that for remote indentations, indicating higher mobile dislocation density in the grain boundary vicinity.

Wherein, Hall-Petch dislocation pile-up model is based on the idea of grain boundary resistance to dislocation transmission due to slip geometry mismatch. Unlike post-yield hardening response envisioned by Ashby-Taylor, this model considers dislocation pile-up in the grain interior against grain boundary until the grain boundary yielding occurs. There is abundant literature to show that the solute concentration affects grain boundary strength. Based on the Gibbs adsorption isotherm, solute segregation at the grain boundary is driven by the energy minimization resulting in stabilizing grain boundary. Such chemistry effects are evident from the Johnson-Cook analysis of remote grain boundary indentations in pure and carbon doped iron, where increase in athermal stresses with decrease in characteristic strain rate for carbon doped remote indentation suggests increase in activation energy required for dislocation transmission and higher solute pinning of dislocations due to solute atoms.

The difference observed in the remote and local GB indentations in pure iron and carbon doped iron samples, where remote indentation behavior suggests grain boundary strengthening consistent with the Hall-Petch model with solute effect prominently observed at the grain boundary, and local indentation appears to be consistent with the Taylor-Ashby model of work hardening with solute presence having no effect on grain boundary hardening appears to be inconsistent with the idea that the grain boundary solute segregation leads to strengthening according to the alloyed layer model [69-71]. Rather this difference appears to suggest that the grain boundary strengthening is based on the excess free volume stability at the grain boundary region, as proposed by the molecular dynamic studies [66-68]. 


\subsubsection{Summary of grain boundary and grain interior results:}

In this strain rate study, the author presents a novel approach to utilize stress-strain rate response from the nanoindentation hardness relaxation to study local grain boundary deformation mechanism. While hardness-strain rate behavior of grain boundary and grain interior indentations is fitted to Johnson-Cook model, Schoeck-Gibbs models and EvansKocks models have enabled determination of physical parameters. As shown in the figure (4.17), insignificant variation in the activation volume suggests that the dislocation interaction with different microstructural defects such as grain boundary, solute atoms, and forest dislocations is governed by the same mechanism. The effect of carbon doping athermal stress vs characteristic strain rate behavior is observed in grain interior as well as grain boundary indentations as shown in the figure (4.18). In terms of grain interior results, for carbon doped condition, in addition to the SSD and GND from the indentation, solute doping gives rise to the Cottrell atmosphere hardening effect. At a given indentation strain rate, dislocation pinning effect of solute atoms is evident from the Nix analysis shown in figure (4.19), as the number of immobile dislocations has reduced in comparison to the pure iron grain interior. In absence of thermal stresses, to overcome the Cottrell atmosphere, additional stress is required for bowing out events to occur resulting in higher athermal stress associated with the carbon doped grain interior. This observation is consistent with the Smidt [36] analysis to determine activation energy. Extending the grain interior analysis from Smidt [36] and Nix [59], to the grain boundary indentations results shown in figure (4.18), grain boundary interaction with dislocations is associated with the higher back-stresses. At the interface, dislocation motion is impeded by the slip geometry constraints and grain boundary structure resistance resulting in higher activation energy for dislocations for transmission/emission. The effect on grain boundary-dislocation interaction is enhanced in presence of solute doping, particularly notable at the grain boundary, where dislocations require higher activation energy for slip transmission. The increased resistance to dislocation transmission across grain boundary in presence of carbon doping observed in here for remote indentation is particularly significant given the 
lack of increased hardening in local indentation due to carbon doping. The contrast in chemical effects for local and remote indentation can be analyzed considering two prominent viewpoints on effect of solute segregation at the grain boundary, i.e. alloyed layer theory [69-71] and grain boundary excess free volume concept [66-68]. While grain boundary behavior with and without carbon exhibits significant changes in athermal stress and characteristic strain rate for the remote indentation geometry, the local indentation geometry does not appear to have any solute doping effect on the athermal stress, characteristic strain rate (figure 4.26) or grain boundary hardness-depth relationship (figure 4.27). Local indentation at a depth greater than $200 \mathrm{~nm}$ results in the simultaneous deformation of the adjacent grains while remote indentation requires slip transmission across the grain boundary in order for both adjacent grains to undergo deformation. The difference in carbon doping effect on the two different indentation geometry informs on the mechanism of grain boundary strengthening by segregation. The lack of effect of grain boundary chemistry on the local indentation behavior suggests there is not an alloyed layer of significant thickness at the grain boundary [69-71] which pins lattice dislocations in the grain boundary vicinity. Instead, the chemical effect observed for the remote indentation suggests that the solute atoms stabilize the grain boundary plane at the nanometer scale against slip transmission. Theories that have been proposed related to this concept suggest that solute atoms fill the voids resulted due to atomic mismatch at the boundary i.e., excess free volume, resulting in a grain boundary resistant to structural changes associated with slip transmission. Now as mentioned in the previous section, molecular dynamics research has presented an approach wherein while solute segregated at the grain boundary does not affect the slip geometry, it further enhances stability of the boundary. The grain boundarydislocation interaction is affected by the reduction in the excess free volume, wherein nucleation or emission of dislocations across grain boundary is inhibited. The experimental data presented in this work appears consistent with the molecular dynamic result that the solute doping based grain boundary strengthening can be explained with the help of excess free volume concept [66-68], rather than alloyed layer theory [69-71]. 


\subsubsection{The analysis of Grain Boundary Pop-in Events using the Johnson-Cook model:}

Grain boundary pop-in event, as explained in the introduction section, are considered to be manifestation of the grain boundary deformation or yielding event. There is abundant literate available to report grain boundary associated pop-in events in various pure metals and alloys [ 7, 23, 32-34]. The factors affecting occurrence of pop-in events include dislocation density, solute segregation, surface roughness, indenter-grain boundary cross-over. Despite critical analysis of grain boundary pop-in events to examine local grain boundary deformation process, the underlying physical mechanism is far from clear [27, $29,35,72]$.

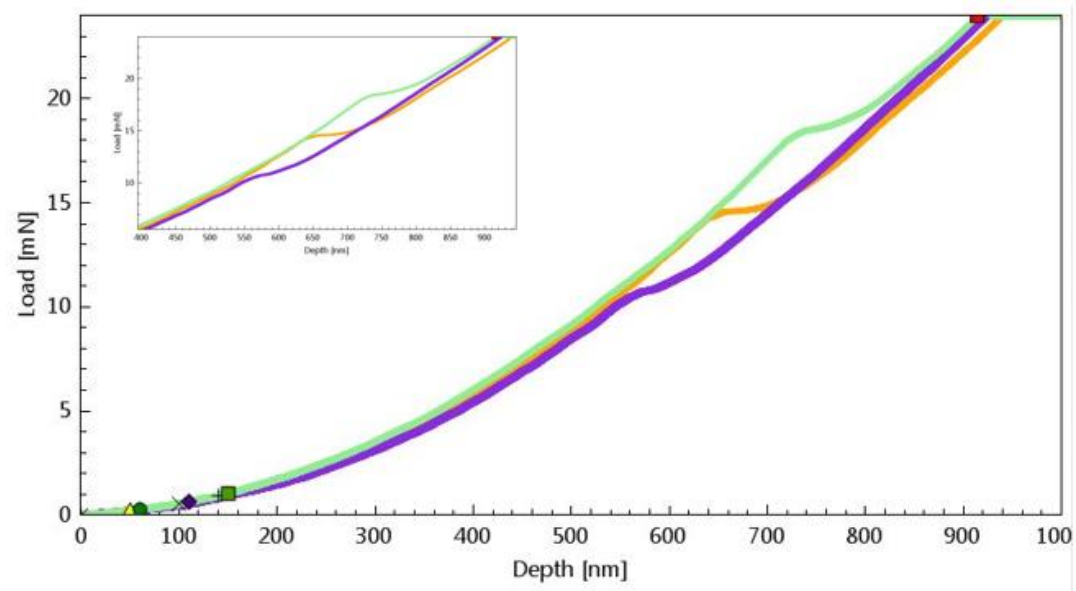

Figure 4.28. A representative load-depth profile for grain boundary indentations with loading pop-ins.

In this chapter, the grain boundary pop-in events observed in case of pure iron and carbon doped iron are discussed in terms of the Johnson-Cook parameters, to examine a role of solute segregation in the grain boundary deformation kinetics. 
A systematic study of grain boundary indentation that exhibited pop-in during loading segment was carried out to determine the physical mechanism responsible for the pop-in. In this analysis, using SEM images, defined Berkovich indenter geometry and the experimental hardness-depth results, a strong correlation was observed between the theoretical depth corresponding to the grain boundary - indenter cross-over and the experimentally observed depth of pop-in. The example of such analysis is presented through figure (4.29), which confirmed that the pop-in events in both, pure iron as well as carbon doped iron grain boundaries, are triggered by the introduction of surface dislocations in the opposite grain.

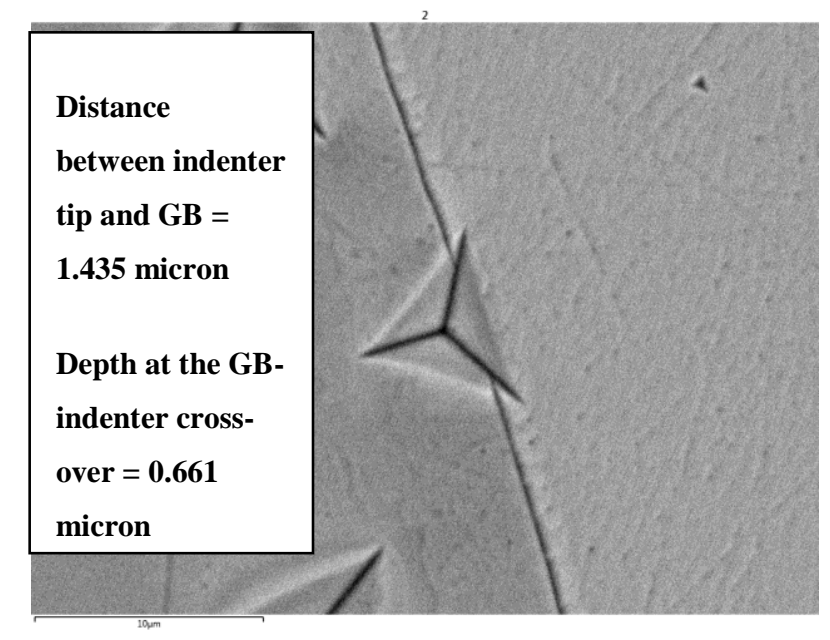

(a)

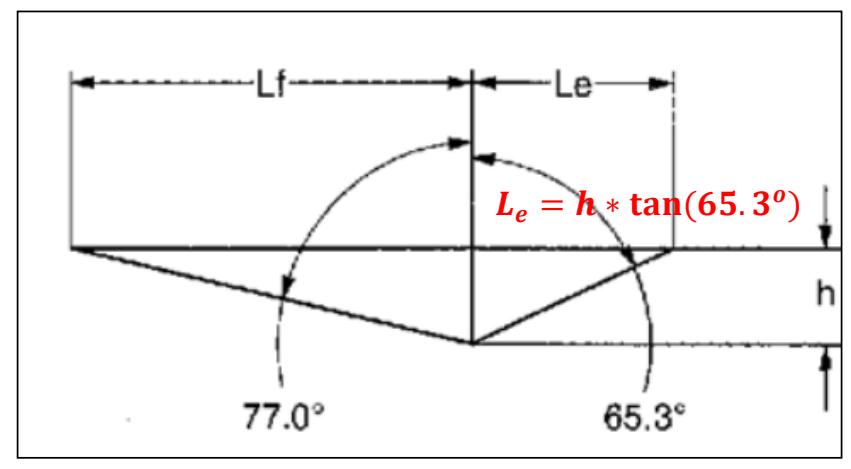

(b) 


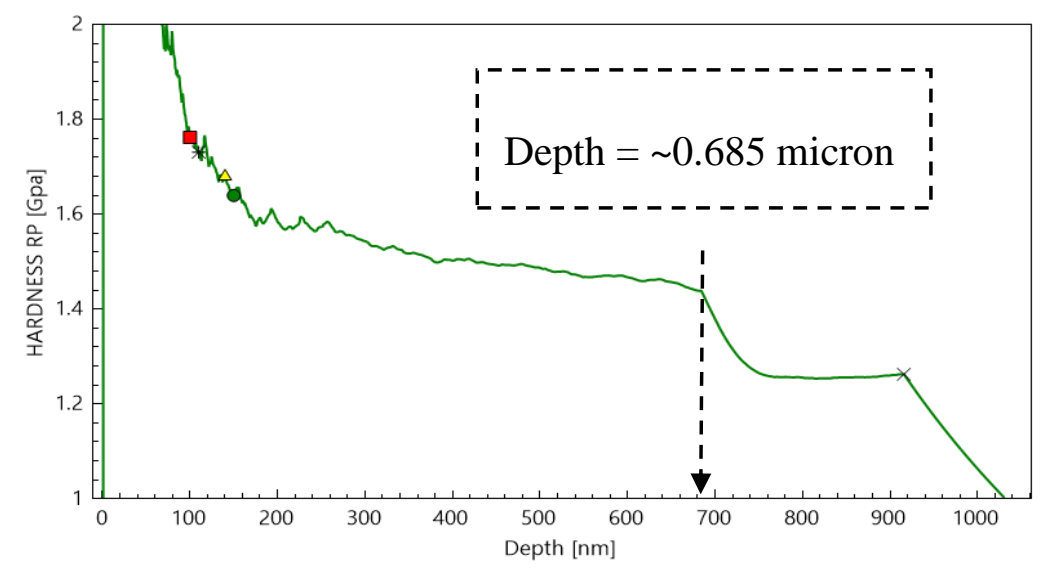

(c)

Figure 4.29. (a) Back-Scatter Electron (BSE) image of grain boundary indentation with remote indentation geometry relative to the grain boundary, (b) Schematic for cross section of Berkovich indenter [73], (c) Hardness vs depth profile for GB indent shown in (a), where pop-in occurs at a depth $\sim 685 \mathrm{~nm}$.
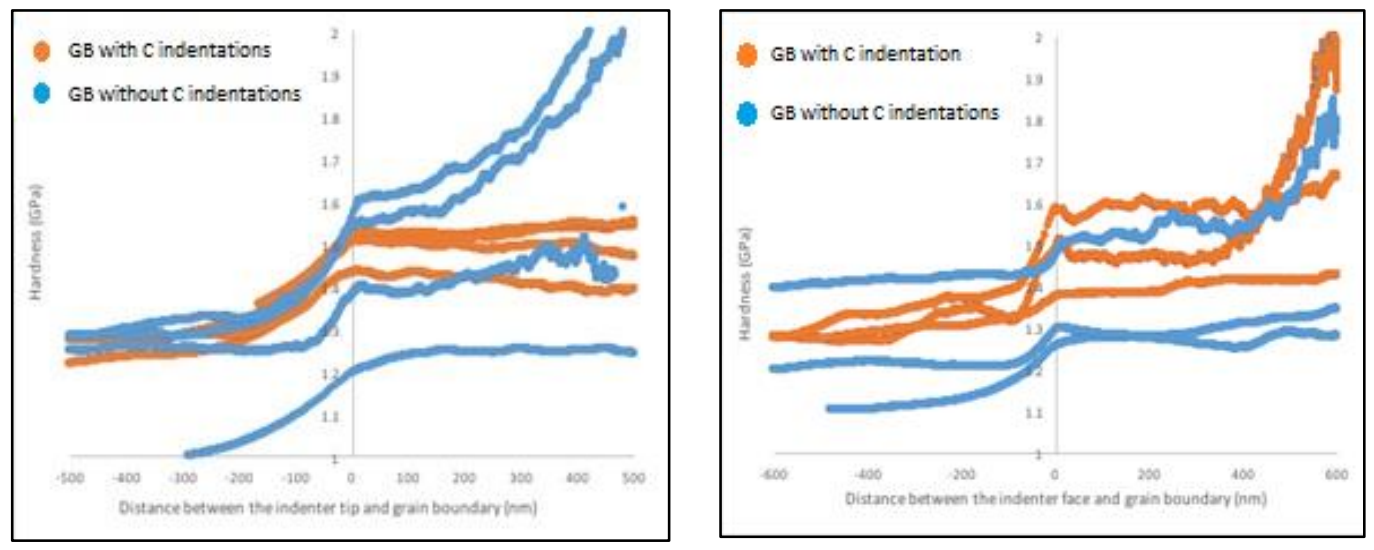

Figure 4.30. Experimental grain boundary pop-in data to show that the pop-in occurs as the indenter face crashes into the grain boundary in with and without carbon cases, (a) for GB1 and (b) GB2. 
The grain boundary pop-in were observed in GB1 and GB2, in with and without carbon conditions. The analysis similar to the one presented in figure (4.29) was carried out for all such indentations, to examine if grain boundary-indenter cross-over coincides with the depth at pop-in. It is clear from the figure (4.30) and (4.31) that the occurrence of pop-in is closely associated with the physical cross-over.

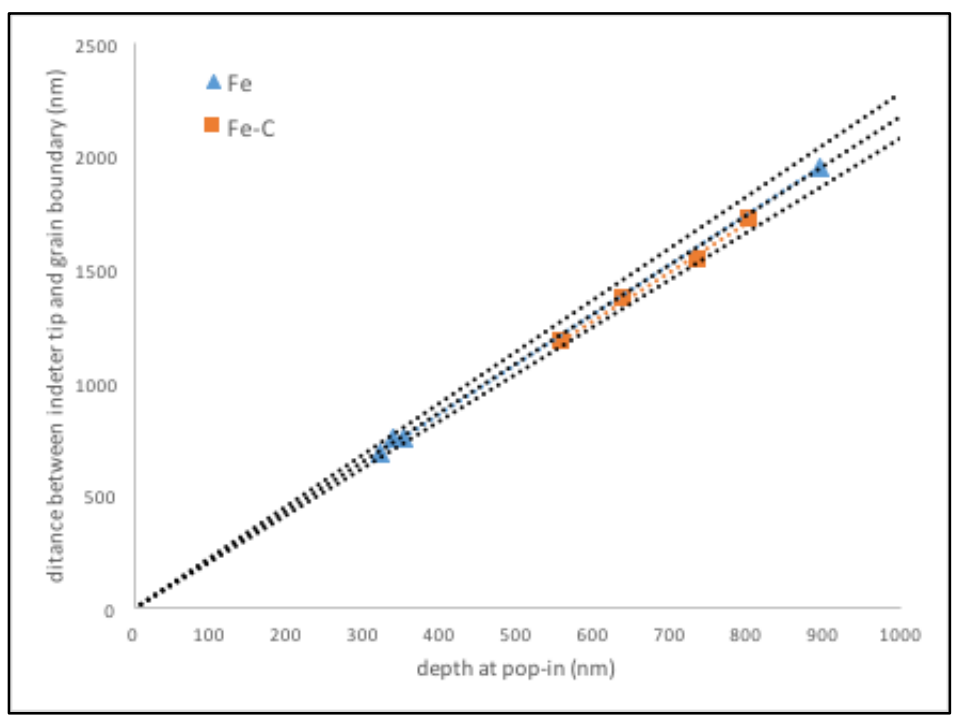

Figure 4.31. Distance between GB - indenter tip (to determine theoretical depth of physical indenter-GB cross-over and the experimental depth of pop-in, for GB1 and GB2.

The physical mechanism of the pop-ins and kinetics of grain boundary deformation is then analyzed through the Johnson-Cook analysis of stress-strain rate response during pop-in event. In this analysis, the Johnson-Cook model is slightly modified to facilitate determination of physical parameters i.e., athermal stress. It is hypothesized that as the indenter crosses the grain boundary, it activates the dislocation source from the opposite grain interior. The grain boundary state of stress is changed due to the surface dislocations introduced the opposite grain interior, in addition to the existing indentation induced 
dislocation pile up. Following analysis presents the approach used to determine variation in the athermal stress associated with the grain boundary pop-in.

As shown in the figure (4.32), the select hardness-depth data corresponding to the pop-in event is utilized to estimate strain rate-hardness response. The difference between the hardness-relaxation during the constant load segment and pop-in event is that the popin event is manifested as a discontinuity in the hardness-depth that occurs within less than a second unlike 60 second duration for the indentation relaxation stress. The application of the Johnson-Cook model to analyze pop-in event is justified as the drop in hardness occurs at a constant load.

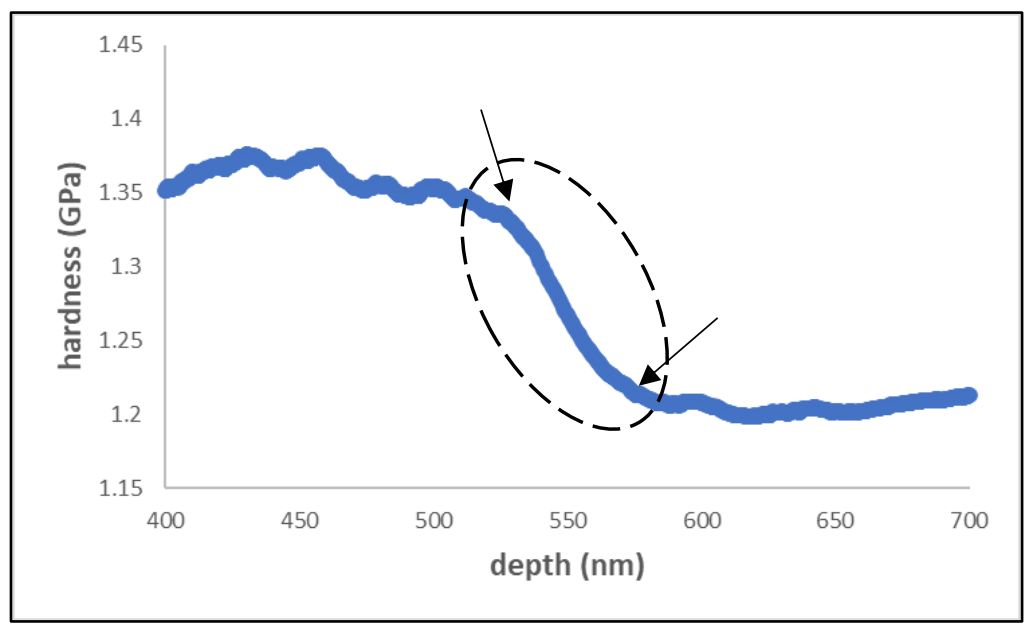

Figure 4.32. A representative hardness-depth profile with hardness drop corresponding to the grain boundary pop-in.

The modified Johnson-Cook approach presented here allows determination of athermal stress as a function of depth, as shown in the equation (4.30). This analysis is based on the assumption that activation volume and characteristic strain rate remains constant and are obtained from the constant load stress relaxation test from the same indentation. 


$$
\sigma_{o}(h)=\sigma-\left(\frac{K T}{V} * \ln \left(\frac{\dot{\varepsilon}_{h}}{\dot{\varepsilon}_{o}}\right)\right)
$$

Where, $\sigma$ - hardness, $\dot{\varepsilon_{h}}=\frac{1}{h} * \frac{d h}{d t}, \dot{\varepsilon}_{o}$ and $V$ - characteristic strain rate and activation volume from the stress relaxation test of the same indentation.

The athermal stress estimated from the JC analysis is plotted against time as shown in figure (4.33), for with and without carbon indentations, to be able to examine stress drop kinetics. The athermal stress vs time response is fitted to the hyperbolic tangent function, and associated parameters such as amplitude, internal decay length are measured for all the grain boundary pop-ins in pure and carbon doped iron, to characterize chemistry effects.

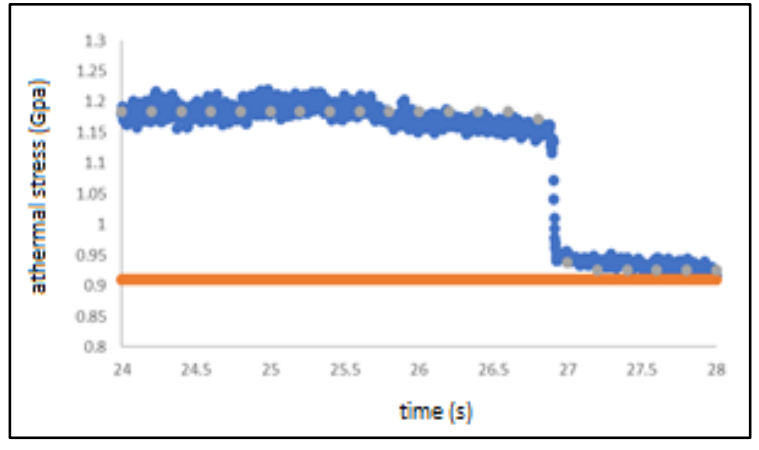

(a)

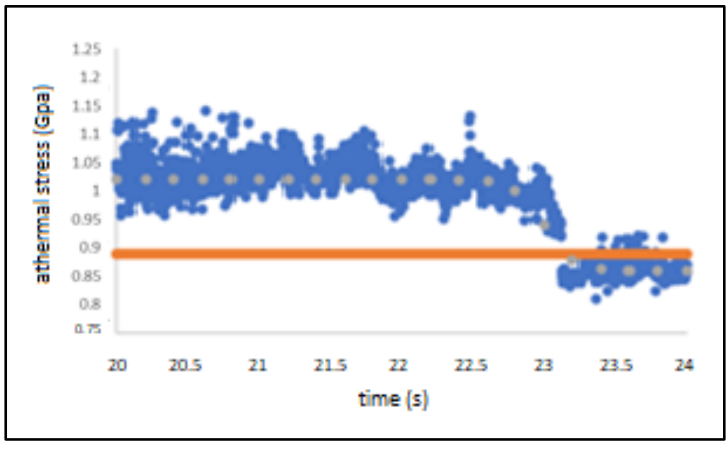

(b)

Figure 4.33. Grain boundary relaxation event observed during indentation loading segment in case of (a) carbon doped iron, (b) pure iron.

After analyzing all the pop-in events at the grain boundaries, GB1 and GB2, the average athermal stress vs time profiles are presented in the figure (4.33). The fitting paramter, $\mathrm{A}$ is a measure of drop in athermal stress during pop-in, B is proportional to the rate of stress drop. 
Comparing with and without carbon pop-in events at these boundaries, it is eveident that the drop in athermal stress is considerably higher for with carbon GB indentations. As

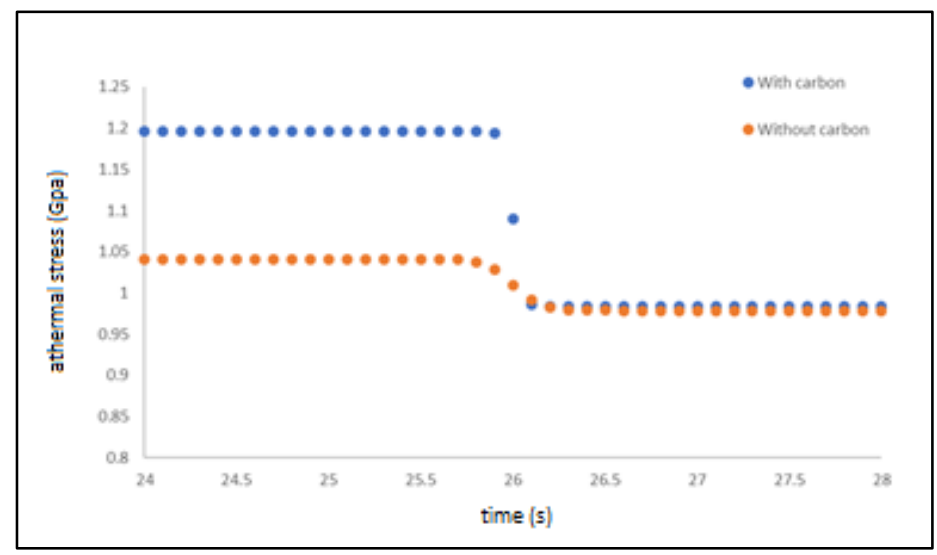

(a)

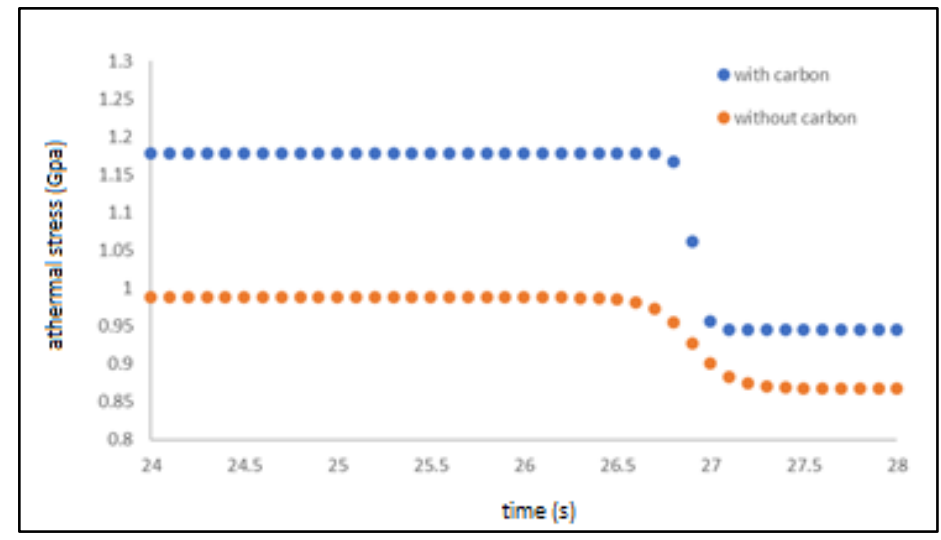

(b)

Figure 4.34. Representative behavior of athermal stress with and without carbon grain boundary pop-ins as a function of time, based on the average data from "tanh" fitting, for (a) GB1 and (b) GB2. 
shown in the table (4.10) and (4.11), magnitude of A and B is higher for carbon doped grain boundaries than pure grain boundaries. These results suggest that the amount of athermal stress or back-stress at the grain boundary with carbon appears to be higher than

\begin{tabular}{|c|c|c|c|c|c|c|}
\hline \multicolumn{7}{|c|}{ Hyporbolic tangent function $-\sigma_{o}=A * \tanh \left[B *\left(t-t_{o}\right)+C\right]$} \\
\hline & \multicolumn{7}{c|}{ Fitting parameters } \\
\cline { 2 - 7 } & $\mathrm{A}$ & $\mathrm{B}$ & $\mathrm{C}$ & $\mathrm{A}$ & $\mathrm{B}$ & $\mathrm{C}$ \\
\hline & 0.106 & 14.57 & 1.09 & 0.0313 & 6.75 & 1.01 \\
\hline Average & 0.02058 & 4.79086 & 0.03302 & 0.04458 & 2.87228 & 0.07348 \\
\hline Std dev & & & & & \\
\hline
\end{tabular}

Table 4.10. Hyperbolic tangent function fitting parameters for the athermal stress vs time plots for GB1 with and without carbon.

\begin{tabular}{|c|c|c|c|c|c|c|}
\hline \multicolumn{7}{|c|}{ Hyporbolic tangent function $-\sigma_{o}=A * \tanh \left[B *\left(t-t_{o}\right)+C\right]$} \\
\hline & \multicolumn{6}{|c|}{ Fitting parameters } \\
\hline & \multicolumn{3}{|c|}{ GB4 with carbon } & \multicolumn{3}{|c|}{ GB4 without carbon } \\
\hline & A & B & $\mathrm{C}$ & A & B & $\mathrm{C}$ \\
\hline Average & 0.012 & 22.8 & 1.062 & 0.06 & 4.83 & 0.928 \\
\hline Std dev & 0.03365 & 5.84807 & 0.06683 & 0.03162 & 2.63944 & 0.05382 \\
\hline
\end{tabular}

Table 4.11. Hyperbolic tangent function fitting parameters for the athermal stress vs time plots for GB2 with and without carbon. 
that for the pure iron, which further leads to higher drop in athermal stress upon pop-in event. The carbon segregation at the grain boundary affects the dislocation-grain boundary interactions wherein solute atoms enhance dislocation pinning until the critical stress is reached or grain boundary deformation is initiated.
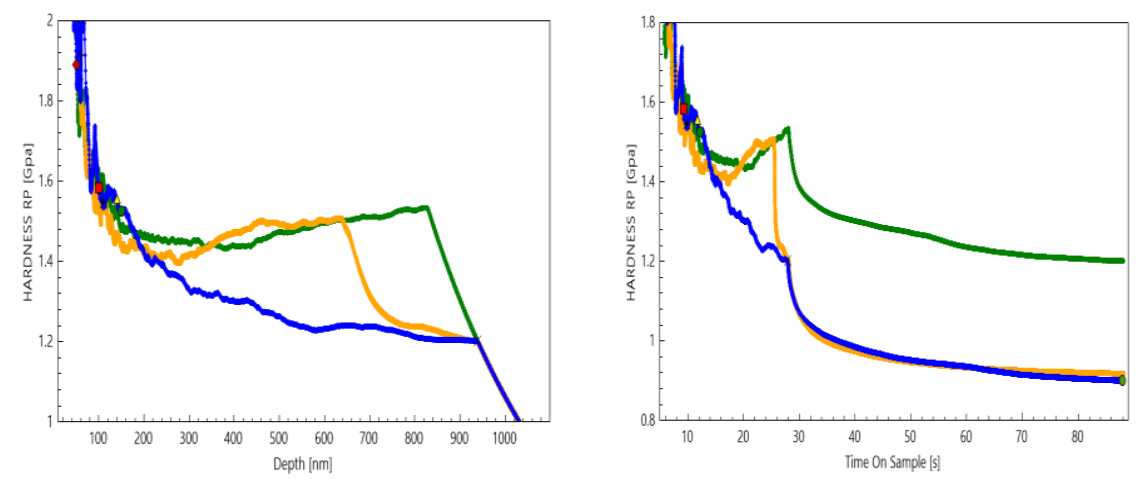

(a)
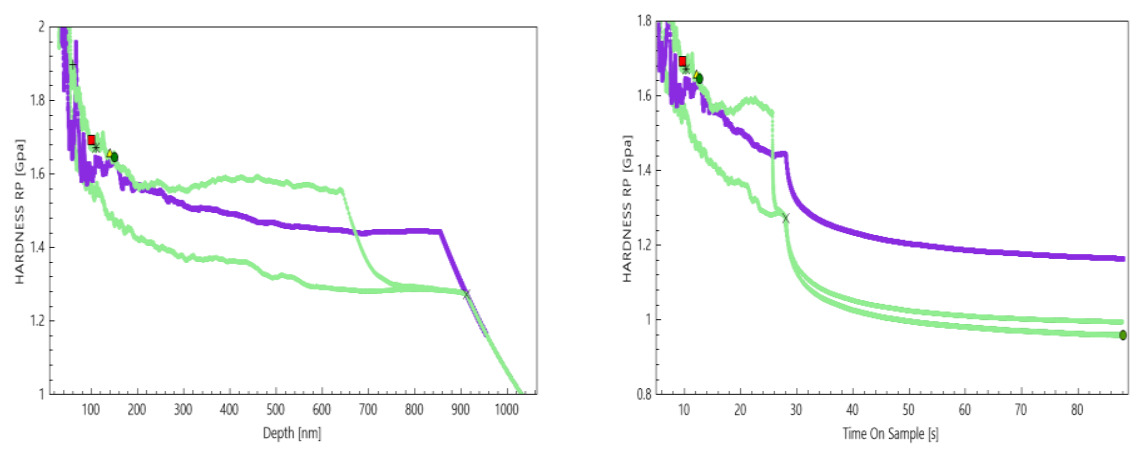

(b)

Figure 4.36. A representative hardness-depth and hardness-time profiles for grain boundary indentations with and without pop-in with reference to the grain interior.

The athermal stress variation during pop-in events are examined with reference to the grain interior stress-strain rate response. As shown in the figure (4.36), a comparison of hardness-time profile for grain boundary indentations with and without pop-in with 
reference to the adjacent grain interior shows that the stress-strain rate response of the boundary after pop-in event closely resembles that of the grain interior. This can be viewed at the effective resistance at the boundary due to slip system mismatch appears to be vanished and it becomes transparent to the dislocation motion.

The chemistry effects on the grain boundary pop-in behavior can be analyzed utilizing Sinclair argument that the dislocation build up at the boundary results in the backstresses [74]. An efficiency of the grain boundary to resist dislocation transmission dependes dislocation pinning potential. The higher stress drop in addition to the dislocation avalanche observed in case of carbon doped grain bboundary pop-in events appear to be strongly affected by the solute asssiated dislocation pinning at the boundary.

\subsection{CONCLUSION:}

To summarize chapter 4 findings as well as the overall project, the original contributions of significance to the field from this work are:

1. The occurrence of significant extrinsic indentation size effects were found to be correlated with inhomogeneous dislocation distributions, whether these inhomogeneous distributions were present before indentation or as a consequence of indentation. These extrinsic size effects extended beyond the Berkovich shape size effect originally explored by Gao and Nix, although there is a possibility that poor surface preparation for nan-indentation may be confused with the Gao-Nix Berkovich shape size effect. As such, the extrinsic size effect can illuminate the presence of inhomogeneous dislocation distributions either pre-existing or that develop during indentation deformation.

2. For the first time, nano-indentation strain rate and constant load hardness relaxation studies have been applied to obtain information on the local grain boundary mechanical response. It was determined that many of the modelling techniques applied to bulk strain rate analysis were applicable to indentation analysis of grain 
boundary and grain interior. This application has enabled the correlation between local athermal stress (back-stress, internal stress), mobile dislocation density and and activation energy.

3. For the first time, the occurrence of the Ashby-Taylor grain boundary hardening mechanism has been resolved from the action of the Hall-Petch pile-up mechanism by using the local vs remote Berkovich indentation geometry. The difference between these two mechanisms has been further verified by the study of the grain boundary chemistry effects on remote vs local, Berkovich nano-indentation mechanical response. The carbon doping of the grain boundaries significantly effects the hardness of the boundary under remote indentation (Hall Petch) while the chemistry effect is insignificant under local indentation (Ashby-Taylor). This behavior would be expected as the Ashby-Taylor hardening depends only on the incompatibility of the strain at the boundary due to 'crystallographic geometry' changes across the boundary plane and a local increase in carbon concentration at a 5-10 angstrom thick grain boundary plane will not effect that 'crystallographic geometry'. However, remote indentation gradually increases the stress at the boundary as indentation depth increases, where yielding in the indented grain occurs before the adjacent grain. In this case, the dislocations will gradually pile up at the grain boundary, until a critical stress is reached to allow dislocation transmission. In this case, the stabilization of the grain boundary structure by chemical segregation is observed to interfere with this transmission, leading to significantly higher athermal stresses and significantly lower mobile dislocation densities when carbon doped.

4. A novel approach to analyze grain boundary pop-in events using the hardness-strain rate behavior after the pop initiation has shown a rapid athermal stress relaxation process, which we know from studies of the grain boundary hardness relation results is also correlated with an increase in mobile dislocation density. It has been discovered that this rapid reduction in athermal stress (and associated increase in mobile dislocation density) is highly correlated with the cross-over of the indenter 
face with the grain boundary plane. This raises the possibility that the grain boundary pop-in is due to plasticity initiation from the near surface region of the adjacent grain as the indenter face crushes the grain boundary-surface triple point 


\section{REFERENCES}

1. The deformation and ageing of mild steel: III discussion of results, Hall, E.O., Proc. Phys. Soc. B 64, 747, 1951.

2. The cleavage strength of polycrystals, Petch, N. J., Iron Steel Inst. 174, 25, 1953.

3. Petch relation and grain boundary sources, Li, J.C.M., Trans. Metall. Soc. AIME 227, 239, 1963.

4. Hall-Petch Relationship and Dislocation Model for Deformation of UltrafineGrained and Nanocrystalline Metals, M. Kato, Materials Transactions 55 (1), 19-24, 2014.

5. The deformation of plastically non-homogeneous materials, Ashby, M.F., Philos. Mag. 21, 399, 1970.

6. Plastic strain in metals, Taylor, G., J. Inst. Met. 62, 307, 1938.

7. Nanoindentation study of slip transfer phenomenon at grain boundaries, Britton T. B, Randman D, Wilkinson A. J., Journal of Mater Res 24, 607-615, 2009.

8. Review on slip transmission criteria in experiments and crystal plasticity models, E. Bayerschen, A.T. McBride, B.D. Reddy, T. Böhlke, J. Mater. Sci. 51 (5), 2243, 2016.

9. Prediction of slip transfer mechanisms across grain boundaries, T.C. Lee, I.M. Robertson, H.K. Birnbaum, Scr. Metall. 23 (5), 799, 1989.

10. The plastic deformation of polycrystalline aggregates, Armstrong, R.W., Codd, I., Douthwaite, R.M., and Petch, N.J., Philos. Mag. 7, 4558, 1962.

11. Ductile-brittle transition in steels irradiated with neutrons, Hull, I. L. Mogford, The Philosophical Magazine: A Journal of Theoretical Experimental and Applied Physics, 3 (35), 1213-1222, 1958.

12. Creep in face-centered cubic metals with special reference to copper, P. Feltham, J. D. Meakin, Acta Metallurgica 7(9), 614-627, 1959.

13. The effect of temperature and grain size on the yield point of copper solid solutions, W. Koster and M. O. Speidel, Z. Metallkunde 56, 585, 1965. 
14. Deformation behavior of nanocrystalline body-centered cubic iron with segregated, foreign interstitials, A. T. Almotasem, M. Posselt, T. Polcar, Materials 13 (23) 1-14, 2020.

15. Effect of interstitial elements on Hall-Petch coefficient of ferritic iron, K. Takeda, N. Nakada, T. Tsuchiyama, S. Takaki, ISIJ International 48 (8), 1122-1125, 2008.

16. Effect of phosphorous on Hall-Petch coefficient in ferritic steel, N. Nakada, K. Takeda, T. Tsuchiyama, S. Takaki, ISIJ International 51 (7) 1169-1173, 2011.

17. Effect of grain boundary segregation of interstitial elements on hallpetch coefficient in steels (Review), Materials Transactions 55(1), 28-34, 2014.

18. Nanoindentation-induced deformation behavior in the vicinity of single grain boundary of interstitial free steel, T. Ohmura, K. Tsuzaki, F. Yin, Materials Transactions 46 (9), 2026-2029, 2005.

19. Grain boundary effect on nanoindentation: A multiscale discrete dislocation dynamics model Songjiang Lu, Bo Zhang, Xiangyu Li, Junwen Zhao, Michael Zaiser, Haidong Fand, Xu Zhang, Journal of the mechanics and physics of solids 126, 117135, 2019.

20. Toward a further understanding of intermittent plastic responses in the compressed single/bicrystalline micropillars, H. Fan, Z. Li, M. Huang, Scripta Mater. 66 (10), 813-816, 2012.

21. Interactions between dislocations and grain boundaries, W.A. Soer, Groningen: University of Groningen, 2016.

22. The mechanical behavior during nanoindentation near the grain boundary in a bicrystal FCC metal, G.Z. Voyiadjis, C. Zhang, Mat. Sci. Eng. A 621, 218-228, 2015.

23. Interfaces within strain gradient plasticity: theory and experiments, Aifantis KE, Soer WA, De Hosson JTM, Willis JR, Acta Mater 54, 5077-5085, 2006.

24. Rate-dependent size effects and material length scales in nanoindentation near the grain boundary for a bicrystal FCC metal, C. Zhang, G.Z. Voyiadjis, Mater. Sci. Eng. A 659, 55-62, 2016. 
25. Crystal plasticity FEM study of nanoindentation behaviors of $\mathrm{Cu}$ bicrystals and $\mathrm{Cu}-$ Al bicrystals, M. Liu, C. Lu, K.A. Tieu, K. Zhou, J. Mater. Res. 30 (16), 2485-2499, 2015.

26. Pre- and post-buckling behavior of bi-crystalline micropillars: Origin and consequences, C. Kirchlechner, F. Toth, F.G. Rammerstorfer, F.D. Fischer, G. Dehm, Acta Mater. 124, 195-203, 2017.

27. Local analysis on dislocation structure and hardening during grain boundary pop-ins in tungsten, Javaid, Y. Xu, K. Durst, J. Mater. Sci. 55 (22), 9597, 2020.

28. Incipient plasticity during nanoindentation at grain boundaries in body-centered cubic metals, W.A. Soer, K.E. Aifantis, J.T.M. De Hosson, Acta Mater. 53 (17), 4665, 2005.

29. Grain boundary pop-in, yield point phenomenon and carbon segregation in aged low carbon steel, Z. Shen, B. wang, G. Liang, Y. Zhang, K. Han, C. Song, ISIJ international 58 (2), 373-375, 2018.

30. Analysis of local grain boundary strengthening utilizing the extrinsic indentation size effect, P. P. Soman, E. G. Herbert, K. E. Aifantis, S. A. Hackney, Journal of Materials Research, 34 (13), 2347-2369, 2019.

31. Nanoindentation-Induced Deformation Behavior in the Vicinity of Single Grain Boundary of Interstitial-Free Steel, Takahito Ohmura, Kaneaki Tsuzaki and Fuxing Yin, Materials Transactions 46 (9), 2026-2029, 2005.

32. Detection of grain-boundary resistance to slip transfer using nanoindentation, Soer WA, De Hosson JThM, Mater Lett 59, 2478-2486, 2005.

33. Indentation strain burst phenomenon induced by grain boundaries in niobium, Wang MG, Ngan AHW, J Mater Res 19, 2478-2486, 2004.

34. Mechanical Characterization of grain boundary using nanoindentation, Kalidindi S.R. Vachhani S. J, Curr. Opin. Solid State Mater Sci 18, 196-204, 2014.

35. Pop-in behavior and elastic-to-plastic transition of polycrystalline pure iron during sharp nanoindentation, Pöhl, F, Sci Rep 9, 15350, 2019. 
36. The Effect of Temperature and Strain-Rate on Thermally Activated Deformation in High Purity Alpha Iron, F. A. Smidt, Jr. Bnwl-184 Uc-25, Metals Ceramics, And Materials (1965).

37. Internal stresses arising from the interaction of mobile dislocation, Argon, A.S., Scripta Metallurgica 4, 1001, 1970.

38. Stress relaxation and creep in $\alpha$-iron filamentary single crystals at low temperature, E Groh and R. Conte, Acta Metallurgica 19 (9), 895-902, 1971.

39. Stress Relaxation and the Plastic Deformation of Solids, E Guiu and E L. Pratt, Phys. Stat. Sol., 6 (1), 111-120, 1964.

40. Creep and stress relaxation studies with polycrystalline magnesium, G. B. Gibbs, The Philosophical Magazine: A Journal of Theoretical Experimental and Applied Physics, 13 (122), 317-329, 1966.

41. Stress relaxation and the structure size-dependence of plastic deformation in nanotwinned copper Lei Lu, Ting Zhu, Yongfeng Shen, Ming Dao, K. Lu, Subra Suresh, Acta Materialia 57 (17), 5165-5173, 2009.

42. Thermal activated mechanisms in crystal plasticity, Caillard D, Martin JL., Amsterdam: Pergamon, 2003.

43. A constitutive model and data for metals subjected to large strains, high strain rates and high temperature, G. Johnson and W. Cook, Seventh international symposium of ballistics, Hague, Netherlands, 1983.

44. A constitutive description of the deformation of copper based on the use of the mechanical threshold, Follansbee, P.S., Kocks, U.F., Acta Metallurgica 36(1), 8193, 1988.

45. Dislocation-mechanics-based constitutive relations for material dynamics calculations, Zerilli, F.J.; Armstrong, R.W., Journal of Applied Physics 61(5), 1816, 1987.

46. Nanoindentation of high-purity vapor deposited lithium films: A mechanistic rationalization of diffusion-mediated flow, E. Herbert, S. Hackney, V. Thole, N. Dudney, P. Phani, Journal of Materials Research, 33 (10), 1347-1360, 2018. 
47. The Activation Energy of Dislocation Movement, G. Schoeck, phys. stat. sol. 8, 499, 1965.

48. The Thermodynamics of Creep Deformation, G. B. Gibbs, Phys Stat 5, 693, 1964.

49. The Thermodynamics of Thermally Activated Dislocation Glide, G. B. Gibbs, Phys. Stat. 10, 507, 1965.

50. The activation parameters for dislocation glide, G. B. Gibbs, Phil. Mag. 16, 97, 1967.

51. Thermally activated deformation of crystalline materials, Evans, A.G. and R.D. Rawlings, Phys. Stat. Sol. 34, 9-31, 1969.

52. Progress in materials science, Kocks, U.F., A.S. Argon, and M.F. Ashby, Prog. Mat. Sci. 19, 1, 1975.

53. Thermally-activated constitutive model including dislocation interactions, aging and recovery for strain path dependence of solid solution strengthened alloys: Application to AA5754-O, Pham, M.S., Mark Iadicola, Adam Creuziger, Lin Hu, Anthony D.Rollett, Int. J. Plasticity, 75, 226-243, 2014.

54. The Role of Carbon Film Thickness in Electron Microprobe Analysis, Derrill M. Kerrick, Leland B. Eminhizer, James F. Villaume, American Mineralogist, 58 (9-10), 920-925, 1973

55. Volume and grain boundary diffusion of $14 \mathrm{C}$ in $\alpha-\mathrm{Fe}, \mathrm{E}$. Budke, Ch. Herzig, $\mathrm{H}$. Wever, Phys Stat Sol., 127, 87, 1991.

56. Grain Boundary Diffusion in Metals: Recent Developments, Herzig, C., and Divinski, S. V., Materials Transactions, 44 (1), 14-27, 2003.

57. Concentration contours in grain boundary diffusion, Whipple, T. P., Phil. Mag., 45, $1225,1954$.

58. Calculation of Diffusion Penetration Curves for Surface and Grain Boundary Diffusion, Fisher, J. C., J. Appl. Phys., 22, 74, 1951.

59. A model for the mobile dislocation density, R. Gasca Neri, W. D. Nix, Acta Metallurgica 22, 257-264, 1974.

60. Thermally activated Deformation of BCC Metals and Alloys, G. Taylor, Progress in Materials Science 36, 29-61, 1992. 
61. Low-temperature deformation of body-centered cubic metals I. Yield and How stress measurements - part I, J. W. Christian and B. C. MASTERS, Proc. R. Soc. A 281, 223, 1964.

62. Low-temperature deformation of body-centered cubic metals I. Yield and How stress measurements - part II, J. W. Christian and B. C. MASTERS, Proc. R. Soc. A 281, 240, 1964.

63. In situ TEM measurement of activation volume in ultrafine grained gold, Saurabh Gupta, Sandra Stangebye, Katherine Jungjohann, Brad Boyce, Ting Zhu , Josh Kacher and Olivier N. Pierron, Nanoscale, 12, 7146-7158, 2012.

64. Thermal activation parameters of plastic flow reveal deformation mechanism in the CrMnFeCoNi high entropy alloy, G. Laplanche, J. Bonneville, C. Varvenne, W.A. Curtin, E.P. George, Acta Materialia, 2017.

65. On Peierls Nabarro stress in Iron, K. T. Kashyap, A. Bhat, P. Koppad, K. B Puneeth, Computation Materials Science, 56, 172-173, 2012.

66. Emission of Dislocations from Grain Boundaries and Its Role in Nanomaterials, Li, J.C.M.; Feng, C.R.; Rath, B.B., Crystals, 11, 41, 2021.

67. Combined effects of nonmetallic impurities and planned metallic dopants on grain boundary energy and strength, Zhifeng Huang, Fei Chen, Qiang Shen, Lianmeng Zhang, Timothy J. Rupert, Acta Materialia, 166, 113-125, 2019.

68. Energy of slip transmission and nucleation at grain boundaries. Sangid, Michael \& Ezaz, Tawhid \& Sehitoglu, Huseyin \& Robertson, I., Acta Materialia, 59, 283-296, 2011.

69. Mechanism of alloy strengthening in refractory metals, R. W. Armstrong, J. H. Bechtold, R. T. Begley, Refractory metals and alloys II, 17, 159-190, 1963.

70. A Study on the Coupled Effects of Solute and Grain Size on the Work Hardening of Fine-Grained FCC Alloys, Ashkan Shadkam, PhD Thesis, 2014, U. British Columbia.

71. Understating the factors influencing yield strength on $\mathrm{Mg}$ alloys, Toda-Caraballo, I., Galindo-Nava, E. I., \& Rivera-Dí, a. P. E., Acta Materialia, 75, 287-296, 2014. 
72. Dislocation-grain boundary interactions: recent advances on the underlying mechanisms studied via nanoindentation testing, Farhan Javaid, Habib Pouriayevali \& Karsten Durst, Journal of Materials Research, 2021.

73. Longitudinal hardness and Young's modulus of spruce tracheid secondary walls using nanoindentation technique, R. Wimmer, B. N. Lucas, W. C. Oliver, T. Y. Tsui, Wood Science and Technology 31, 131-141, 1997.

74. A model for the grain size dependent work hardening of copper, C. W. Sinclair, W. J. Poole, Y. Brechet, Scripta Materialia, 55, 739-742, 2006. 


\section{Appendix 4.A. Activation Volume Measurement}

In this work, the Johnson-Cook model is used in conjunction with Schoeck-Gibbs model to determine activation volume. This method provides an average value of activation volume over the stress relaxation test duration. But the activation volume is an area swept by dislocations under the application of applied stress. During the stress relaxation, the activation volume continues to evolve. In addition to the Johnson-Cook approach, two additional methods are provided below, to study activation volume variation during the indentation constant load segment and stress jump at the end of hardness relaxation.

1. Depth-dependent activation volume measurement during indentation hardness relaxation:

In addition to the traditional approach to determine the apparent activation volume examining the time dependent stress relaxation at constant strain, this study presents a modified approach to determine activation volume using Berkovich indentation stress relaxation (constant load) because of the inherent relationship between indentation depth and hardness, the derivation of [37-39] is carried out in terms of indentation depth rather than time. Similar to the references, we consider the differential of the hardness, but with respect to $\mathrm{h}$ instead of time.

$$
\frac{d \sigma}{d h}=\frac{d}{d h} \sigma_{o}+\frac{K T}{V}\left(\frac{d \ln (\text { strain rate }}{d h}\right)
$$

The hardness in the load hold is determined by the constant load, $\mathrm{P}$, divided by the area function. A simplified area function is used here.

$$
\sigma=\frac{P}{\alpha \cdot h^{2}}
$$


differentiating (4.A.2) and substituting into (4.A.1) gives,

$$
-\frac{2 P}{\alpha \cdot h^{3}}=\frac{d}{d h} \sigma_{o}+\frac{K T}{V}\left(\frac{d \ln (\text { strain rate }}{d h}\right)
$$

Integrating from the initial value of depth when the hold begins, ho, to a particular depth, h, determines.

$$
\int_{h_{o}}^{h}-\frac{2 P}{\alpha \cdot h^{3}} d h=\sigma_{o}(h)-\sigma_{o}\left(h_{o}\right)+\frac{K T}{V}\left(\ln \frac{(\operatorname{strain} r a t e(h)}{\left(\operatorname{strain} \text { rate }\left(h_{o}\right)\right.}\right)
$$

with the final result,

$$
-\frac{P\left(h^{3}-h_{o}{ }^{3}\right)}{\alpha \cdot h^{2} h_{o}{ }^{2}}=\sigma_{o}(h)-\sigma_{o}\left(h_{o}\right)+\frac{K T}{V}\left(\ln \frac{(\operatorname{strain} \text { rate }(h)}{\left(\operatorname{strain} \text { rate }\left(h_{o}\right)\right.}\right)
$$

In equation (4.A.5), all the parameters are experimentally determined during stress relaxation test at constant load, except for $\left[\sigma_{o}(h)-\sigma_{o}\left(h_{o}\right)\right]$ and $\frac{K T}{V} \cdot\left(\ln \left(\frac{\operatorname{StrainRate}(h)}{\operatorname{StrainRate}\left(h_{o}\right)}\right)\right)$. Using combination of all these parameters, over a length of indentation depth measured allows determination of activation volume as a function of depth. 
2. Activation volume measurement using stress jump method:

the stress jump method to determine activation volume is presented in [41-42] as follows:

$$
V^{*}=k_{B} T \frac{\ln \left(\frac{\dot{\gamma}_{i 2}}{\dot{\gamma}_{f 1}}\right)}{\Delta \tau^{*}}
$$

Where, $\left[\dot{\gamma}_{i 2}\right]$ and $\left[\dot{\gamma}_{f 1}\right]$ are the shear strain rate at the onset of relaxation 2 and at the end of relaxation 1 , respectively. This approach considers effect of strain rate sensitivity as well as contribution of mobile dislocation density.

Results are combined for grain boundaries and grain interiors in pure iron and carbon doped iron conditions as presented in the figure (4.A.1).

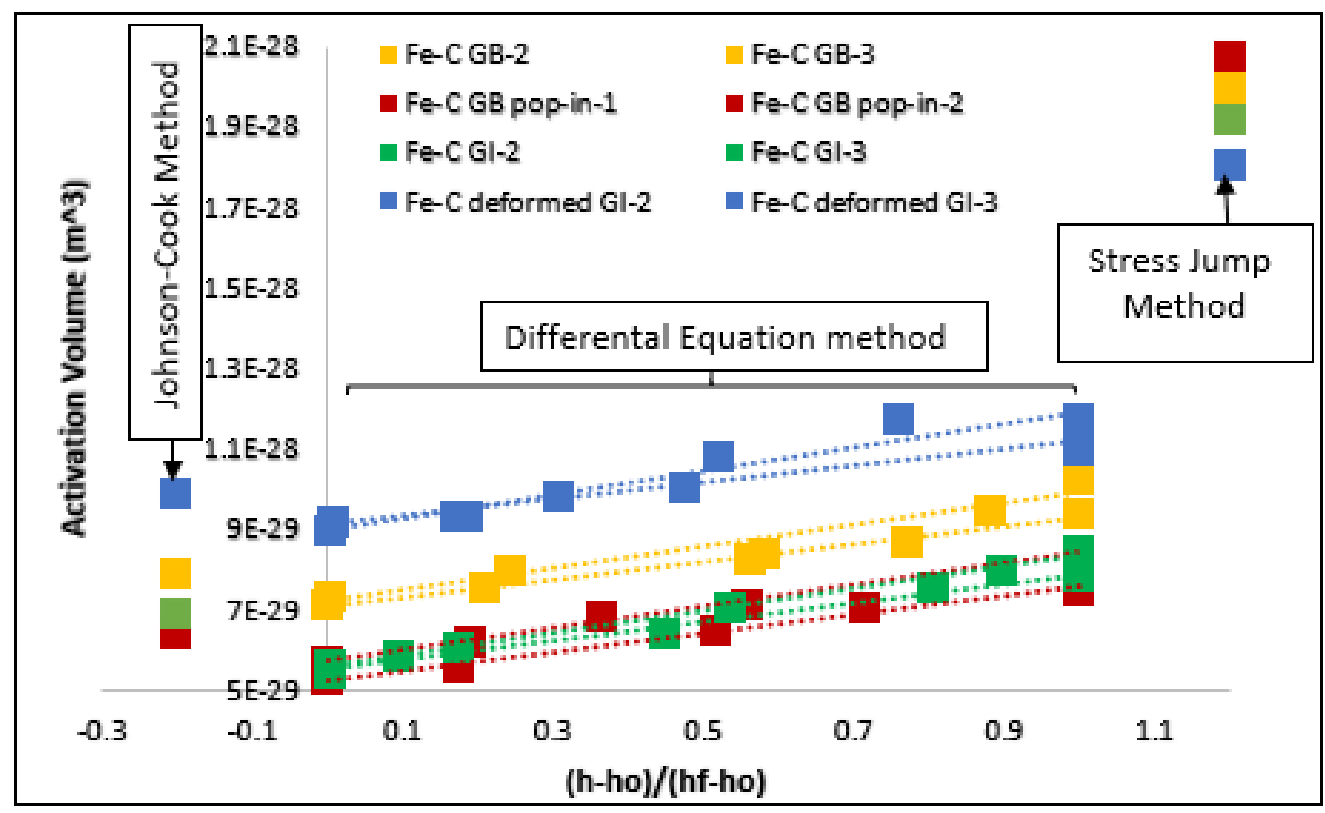


Figure 4.A.1. Activation volume as determined using Johnson-Cook model, Differential equation method for depth dependent activation volume and stress jump method for grain boundaries, grain interiors in pure, carbon doped and deformed conditions. 


\section{Appendix 4.B. Nix Analysis for Mobile Dislocation Density Calculations}

This appendix considers the Neri-Nix analysis for mobile dislocation density as a function of stress [59]. Reexamining Nix with a simple distribution of the number of pinned dislocation segments of length $\mathrm{R}$ given as:

$$
N(R)=A * R *\left(R_{0}-R\right)
$$

The $\mathrm{A}$ and $R_{0}$ parameters are determined by the limiting conditions that the integral of the distribution gives the total dislocation density and that the observed athermal stress corresponds to $\mathrm{R}$ value at the maximum of the distribution. This determines

$$
\begin{gathered}
A=\frac{3 \cdot \rho_{. \operatorname{tot}^{\circ} \cdot \sigma^{3}}}{4 \cdot \mathrm{G}^{3} \cdot \mathrm{b}^{3}} \\
\mathrm{R}_{. \mathrm{o}}=\frac{2 \cdot \mathrm{G} \cdot \mathrm{b}}{\sigma_{.0}}
\end{gathered}
$$

Following Nix [59], the lower limit on the integral for the mobile dislocation density is determined by the pinned dislocation segment length where the applied stress is equal to the back stress, giving the integral 


$$
\rho_{. \text {mobile }}=\int_{\frac{\mathrm{G} \cdot \mathrm{b}}{\sigma}}^{\frac{2 \cdot \mathrm{G} \cdot \mathrm{b}}{\sigma_{. \mathrm{o}}}} \frac{3 \cdot \rho_{. t o t} \cdot \sigma_{.0}^{3}}{4 \cdot \mathrm{G}^{3} \cdot \mathrm{b}^{3}} \cdot \mathrm{R} \cdot\left(\frac{2 \cdot \mathrm{G} \cdot \mathrm{b}}{\sigma_{. \mathrm{o}}}-\mathrm{R}\right) \mathrm{dR}=\frac{\rho_{. \text {tot }} \cdot\left(\sigma_{. \mathrm{o}}-2 \cdot \sigma\right)^{2} \cdot\left(\sigma+\sigma_{. \mathrm{o}}\right)}{4 \cdot \sigma^{3}}
$$

$$
\sigma_{\mathrm{o}}=0.70 \mathrm{GPa}
$$

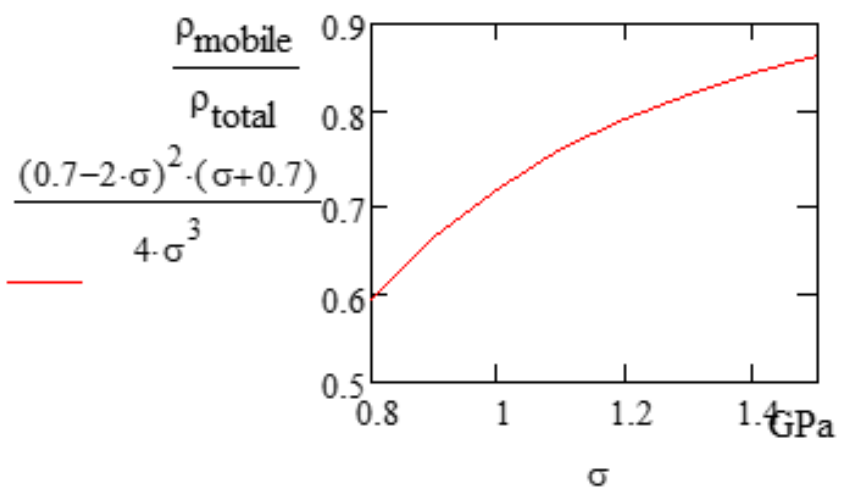

Figure 4.B.1. The normalized mobile dislocation density is plotted as a function of applied stress to show a predicted increase in mobile dislocation density with increasing stress.

The mobile dislocation density as a function of both stress and athermal stress can also be developed from equation (4.B.4) as plotted in figure (4.B.2). Note the use of the Heaviside step function in the mobile dislocation density function in order to disallow results where the athermal stress is greater than the stress. 


$$
\rho_{\text {mobile }}\left(\sigma, \sigma_{\mathrm{o}}\right):=\rho_{\text {total }} \cdot \frac{\left(\sigma_{\mathrm{o}}-2 \cdot \sigma\right)^{2} \cdot\left(\sigma+\sigma_{\mathrm{o}}\right)}{4 \cdot \sigma^{3}} \cdot \Phi\left(\sigma-\sigma_{\mathrm{o}}\right)
$$

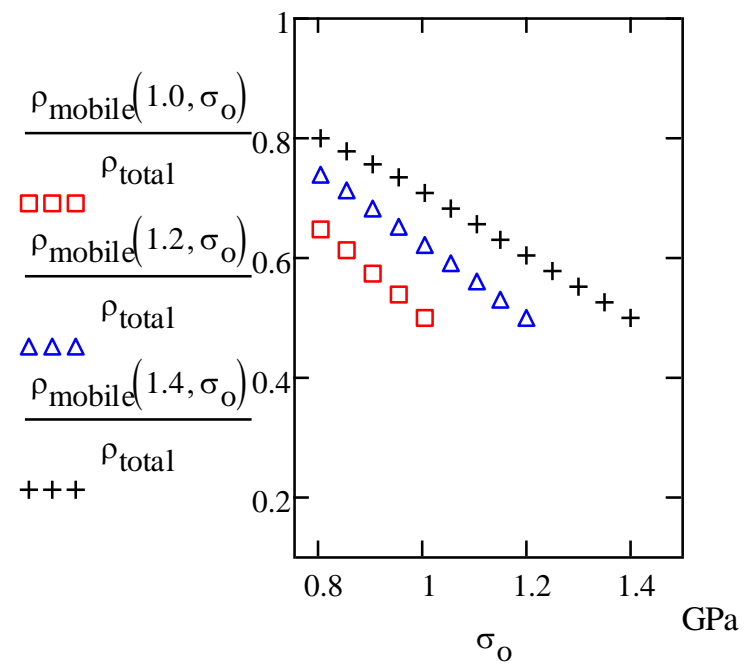

Figure 4.B.2. The calculated normalized mobile dislocation density at different values of stress and athermal stress. 